Supporting Information (SI)

\title{
A Synthesis Strategy for the Production of a Macrolactone of Gulmirecin A via a Ni(0)-Mediated Reductive Cyclization Reaction
}

\author{
Shun Kitahata ${ }^{\mathrm{a}}$, Akira Katsuyama ${ }^{\mathrm{a}, \mathrm{b}}$ and Satoshi Ichikawa ${ }^{* a, b}$ \\ ichikawa@pharm.hokudai.ac.jp
}

${ }^{a}$ Faculty of Pharmaceutical Science, Hokkaido University; Kita-12, Nishi-6, Kita-ku, Sapporo 060-0812, Japan.

${ }^{b}$ Center for Research and Education on Drug Discovery, Hokkaido University, Kita-12, Nishi-6, Kita-ku, Sapporo 060-0812, Japan.

\section{Table of contents}

1) General experimental methods $\quad \mathrm{S} 2$

2) Synthetic schemes for preparation and chemical data of compounds $\quad$ S2-S38

3) ${ }^{1} \mathrm{H}$ and ${ }^{13} \mathrm{C}$ NMR spectra of compounds $\quad$ S39-S123 


\section{1) General experimental methods}

All reactions except that carried out in aqueous phase were performed under argon atmosphere, unless otherwise noted. Materials were purchased from commercial suppliers and used without further purification, unless otherwise noted. Solvents were distilled according to the standard protocol. Isolated yields were calculated by weighing products. The weight of the starting materials and the products were not calibrated. Analytical thin layer chromatography (TLC) was performed on Merck silica gel $60 \mathrm{~F}_{254}$ plates. Normal-phase column chromatography was performed on Merck silica gel 5715 or Wakogel 60N. Flash column chromatography was performed on Kanto Chemical Silica Gel 60N (spherical, neutral, 40-50 $\mu \mathrm{m}$ ). Hi-flash column chromatography was performed on YAMAZEN Hi-Flash ${ }^{\mathrm{TM}}$ column silica gel $(40 \mu \mathrm{m})$ or Fuji Silysia Chromatorex MB/PSQ $(50-200 \mu \mathrm{m})$. All microwave irradiation experiments were carried out in a Biotage ${ }^{\circledR}$ Initiator microwave apparatus with continuous irradiation power from 0 to $400 \mathrm{~W}$ with utilization of the standard absorbance level of $250 \mathrm{~W}$ maximum power. The reactions were carried out in $5 \mathrm{~mL}$ glass tubes, sealed with microwave cavity. The reaction was irradiated at a required ceiling temperature using maximum power for the stipulated time. ${ }^{1} \mathrm{H}$ NMR spectra were measured in $\mathrm{CDCl}_{3}$ or methanol- $d_{4}$ solution and reported in parts per million $(\delta)$ relative to tetramethylsilane $(0.00 \mathrm{ppm})$ as internal standard using JEOL ECS400, ECX400P and ECA500, unless otherwise noted. ${ }^{13} \mathrm{C}$ NMR spectra were measured in $\mathrm{CDCl}_{3}$ or methanol- $d_{4}$ solution and referenced to residual solvent peaks of $\mathrm{CDCl}_{3}(77.0 \mathrm{ppm})$ or methanol- $d_{4}(49.0 \mathrm{ppm})$ using JEOL ECS400, ECX400P and ECA500. Coupling constant $(J)$ was reported in hertz (Hz). Abbreviations of multiplicity were as follows; s: singlet, d; doublet, t: triplet, q: quartet, m: multiplet, br: broad. Data were presented as follows; chemical shift (multiplicity, integration, coupling constant). Assignment was based on ${ }^{1} \mathrm{H}-{ }^{1} \mathrm{H}$ COSY, HMBC and HMQC NMR spectra. Mass spectra were obtained on Waters MICRO MASS LCT-premier and the mass analyzer type used for the HRMS measurements was TOF. Optical rotation was measured on a Rudolph Research Analytical Autopol IV automatic polarimeter.

\section{2) Synthetic scheme for preparation and chemical data of compounds}

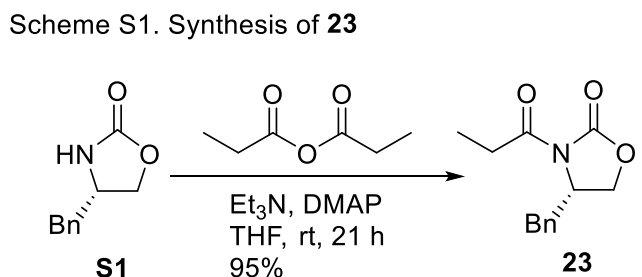

\section{(S)-4-Benzyl-3-propionyl-2-oxazolidinone (23)}

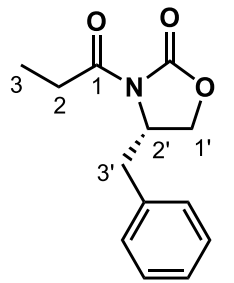

A solution of $\mathbf{S 1}(1.77 \mathrm{~g}, 10 \mathrm{mmol}), \mathrm{Et}_{3} \mathrm{~N}(1.67 \mathrm{~mL}, 12 \mathrm{mmol})$ and DMAP $(61 \mathrm{mg}, 0.50 \mathrm{mmol})$ in THF $(10 \mathrm{~mL})$ was treated with propionic anhydride $(1.82 \mathrm{~mL}, 15 \mathrm{mmol})$ at room temperature for $21 \mathrm{~h}$. The mixture was partitioned between $\mathrm{CH}_{2} \mathrm{Cl}_{2}$ and $\mathrm{H}_{2} \mathrm{O}$. The organic phase was washed with 1 $\mathrm{M}$ aq. $\mathrm{HCl}, 1 \mathrm{M}$ aq. $\mathrm{NaOH}$ and brine, dried $\left(\mathrm{Na}_{2} \mathrm{SO}_{4}\right)$, filtered and concentrated in vacuo. The residue was purified by hi-flash silica gel column chromatography $(\phi 2.6 \times 10 \mathrm{~cm}, 5 \rightarrow 10 \rightarrow 20 \%$ 
EtOAc/hexane) to afford $23(2.2 \mathrm{~g}, 9.5 \mathrm{mmol}, 95 \%)$ as a white solid. ${ }^{1} \mathrm{H} \mathrm{NMR}\left(\mathrm{CDCl}_{3}, 400 \mathrm{MHz}\right) \delta 7.33(\mathrm{~m}, 5 \mathrm{H}, \mathrm{H}-$ Ph), 4.7 (br s, 1H, H-2'), 4.18 (m, 2H, H-2), 3.32 (d, 1H, H-1', $J_{\text {gem }}=13.8$ Hz), 2.96 (m, 2H, H-1', H-3'), 2.76 (m, 1H, $\left.\mathrm{H}^{\prime} 3^{\prime}\right), 1.21\left(\right.$ br s, 3H, H-3); ESIMS-LR $m / z 256\left[(\mathrm{M}+\mathrm{Na})^{+}\right] ;[\alpha]^{20}{ }_{\mathrm{D}}-56.9\left(c 1.00, \mathrm{CHCl}_{3}\right)$. This is a known compound. ${ }^{\mathrm{S} 1}$

Scheme S2. Synthesis of $\mathbf{2 5}$
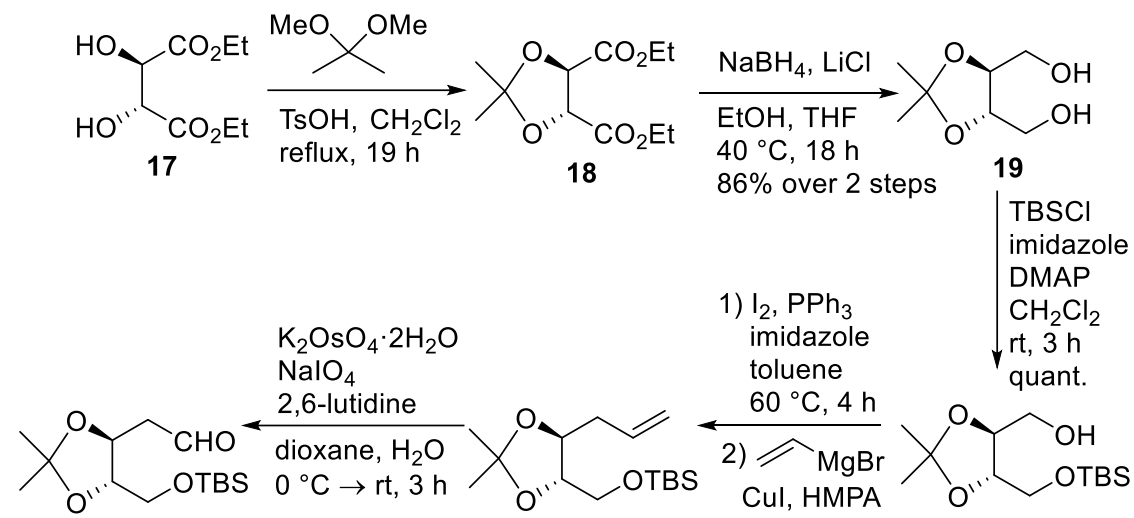

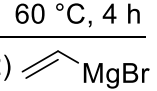

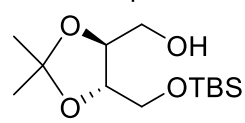

22

21

Cul, HMPA

THF

$-40{ }^{\circ} \mathrm{C} \rightarrow \mathrm{rt}, 8 \mathrm{~h}$

20

23, $\mathrm{TiCl}_{4},{ }^{i} \mathrm{Pr}_{2} \mathrm{NEt}$

1-methyl-2-pyrrolidinone

$83 \%$ over 2 steps

$\mathrm{CH}_{2} \mathrm{Cl}_{2}, 0{ }^{\circ} \mathrm{C}, 2 \mathrm{~h}$

$96 \%$ over 2 steps

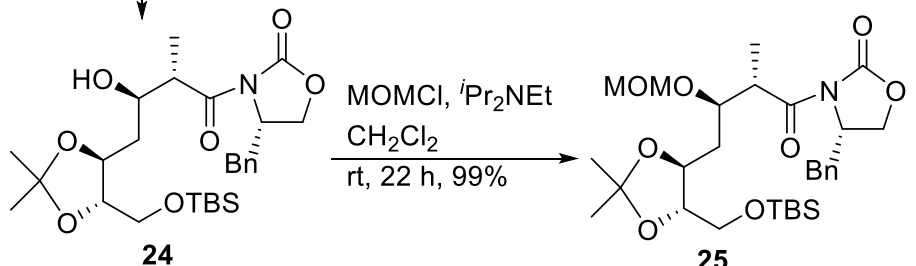

\section{(4S,5S)-2,2-Dimethyl-1,3-dioxolane-4,5-dimethanol (19)}<smiles>CC1(C)O[C@@H](CO)[C@@H]1CO</smiles>

A solution of diethyl L-(+)-tartrate $(20.6 \mathrm{~g}, 100 \mathrm{mmol})$ in $\mathrm{CH}_{2} \mathrm{Cl}_{2}(250 \mathrm{~mL})$ was treated with dimethoxypropane $(37 \mathrm{~mL}, 300 \mathrm{mmol})$ and $\mathrm{TsOH}(380 \mathrm{mg}, 2 \mathrm{mmol})$ at room temperature for $19 \mathrm{~h}$. The mixture was partitioned between $\mathrm{CH}_{2} \mathrm{Cl}_{2}$ and sat. aq. $\mathrm{NaHCO}_{3}$. The organic phase was washed with brine, dried $\left(\mathrm{Na}_{2} \mathrm{SO}_{4}\right)$, filtered and concentrated in vacuo to give 18. A solution of the residue $(22 \mathrm{~g})$ in EtOH $(300 \mathrm{~mL})$ and THF $(150 \mathrm{~mL})$ was treated with $\mathrm{LiCl}(22.7 \mathrm{~g}, 53 \mathrm{mmol})$ and $\mathrm{NaBH}_{4}(20.2 \mathrm{~g}, 53 \mathrm{mmol})$ at $0{ }^{\circ} \mathrm{C}$, and the mixture was warmed to $40^{\circ} \mathrm{C}$ and stirred for $18 \mathrm{~h}$. The reaction was quenched with acetone $(100 \mathrm{~mL})$. The mixture was filtered through a Cerite pad, and the filtrate was concentrated in vacuo. The residue was purified by silica gel column chromatography $\left(\phi 6 \times 10 \mathrm{~cm}, 1 \rightarrow 5 \% \mathrm{MeOH} / \mathrm{CH}_{2} \mathrm{Cl}_{2}\right)$ to afford 19 (14.0 g, $86.3 \mathrm{mmol}, 86 \%$ over 2 steps) as a colorless oil. ${ }^{1} \mathrm{H}$ NMR $\left(\mathrm{CDCl}_{3}, 400 \mathrm{MHz}\right) \delta 4.01(\mathrm{~m}, 2 \mathrm{H}, \mathrm{H}-2), 3.81(\mathrm{~m}, 2 \mathrm{H}, \mathrm{H}-1), 3.70(\mathrm{~m}, 2 \mathrm{H}, \mathrm{H}-1), 2.17$ (t, $\left.2 \mathrm{H}, \mathrm{H}-\mathrm{OH}, J_{\mathrm{OH}, 1}=4.6 \mathrm{~Hz}\right), 1.44\left(\mathrm{~s}, 6 \mathrm{H}, \mathrm{H}-\mathrm{OC}\left(\mathrm{CH}_{3}\right)_{2} \mathrm{O}\right)$; ESIMS-LR $m / z 185\left[(\mathrm{M}+\mathrm{Na})^{+}\right]$. This is a known compound. ${ }^{\mathrm{S} 2}$ 


\section{(4S,5S)-5-tert-Butyldimethylsilyloxymethyl-2,2-dimethyl-1,3-dioxolane-4-methanol (20)}<smiles>CC(O)CO[AsH3]</smiles>

A solution of 19 (10.7 g, $66 \mathrm{mmol})$ in $\mathrm{CH}_{2} \mathrm{Cl}_{2}(220 \mathrm{~mL})$ was treated with imidazole $(4.49 \mathrm{~g}$, $66 \mathrm{mmol})$ and $\mathrm{TBSCl}(9.9 \mathrm{~g}, 66 \mathrm{mmol})$ at $0{ }^{\circ} \mathrm{C}$ for $2 \mathrm{~h}$. Imidazole $(2.3 \mathrm{~g}, 33 \mathrm{mmol})$ and $\mathrm{TBSCl}$ (4.9 g, $33 \mathrm{mmol}$ ) were added to the mixture, which was stirred for $1 \mathrm{~h}$. The mixture was partitioned between $\mathrm{CH}_{2} \mathrm{Cl}_{2}$ and sat. aq. $\mathrm{NaHCO}_{3}$. The organic phase was washed with brine, dried $\left(\mathrm{Na}_{2} \mathrm{SO}_{4}\right)$, filtered and concentrated in vacuo. The residue was purified by silica gel column chromatography $(\phi 6 \times 10 \mathrm{~cm}, 20 \%$ EtOAc/hexane) to afford $20\left(18.1 \mathrm{~g}, 66 \mathrm{mmol}\right.$, quant.) as a colorless oil. ${ }^{1} \mathrm{H} \mathrm{NMR}\left(\mathrm{CDCl}_{3}, 400 \mathrm{MHz}\right) \delta 3.90(\mathrm{dt}, 1 \mathrm{H}$, $\left.\mathrm{H}-2, J_{2,3}=7.8, J_{2,1}=5.0 \mathrm{~Hz}\right), 2.30(\mathrm{~s}, 1 \mathrm{H}, \mathrm{H}-\mathrm{OH}), 1.33\left(\mathrm{~s}, 3 \mathrm{H}, \mathrm{H}-\mathrm{OC}\left(\mathrm{CH}_{3}\right)_{2} \mathrm{O}\right), 1.31\left(\mathrm{~s}, 3 \mathrm{H}, \mathrm{H}-\mathrm{OC}(\mathrm{CH})_{2} \mathrm{O}\right), 0.81(\mathrm{~s}$ $\left.9 \mathrm{H}, \mathrm{H}-{ }^{t} \mathrm{Bu}\right), 0.01\left(\mathrm{~s}, 6 \mathrm{H}, \mathrm{H}-\mathrm{SiCH}_{3}\right)$; ESIMS-LR $m / z 299\left[(\mathrm{M}+\mathrm{Na})^{+}\right]$. This is a known compound. ${ }^{\mathrm{S} 3}$

[(4R,5R)-5-tert-Butyldimethylsilyloxymethyl-2,2-dimethyl-1,3-dioxolan-4-yl] propene (21)

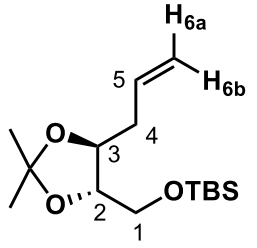

A solution of $20(4.1 \mathrm{~g}, 14.9 \mathrm{mmol})$ in toluene $(150 \mathrm{~mL})$ was treated with imidazole $(3.04 \mathrm{~g}$, $44.7 \mathrm{mmol}), \mathrm{PPh}_{3}(4.69 \mathrm{~g}, 17.9 \mathrm{mmol})$ and $\mathrm{I}_{2}(2.84 \mathrm{~g}, 22.4 \mathrm{mmol})$ at room temperature. The mixture was warmed to $60{ }^{\circ} \mathrm{C}$ and stirred for $4 \mathrm{~h}$. The reaction was quenched with sat. aq. $\mathrm{Na}_{2} \mathrm{~S}_{2} \mathrm{O}_{3}$ at room temperature. The mixture was extracted with hexane. The organic phase was washed with sat. aq $\mathrm{Na}_{2} \mathrm{~S}_{2} \mathrm{O}_{3}$ and brine, dried $\left(\mathrm{Na}_{2} \mathrm{SO}_{4}\right)$, filtered and concentrated in vacuo. A solution of the residue (5.6 g) in THF (100 $\mathrm{mL})$ and HMPA $(50 \mathrm{~mL})$ was treated with $\mathrm{CuI}(141 \mathrm{mg}, 0.74 \mathrm{mmol})$ and vinylmagnesium bromide $(29.6 \mathrm{~mL}, 29.6$ mmol, $1 \mathrm{M}$ in THF) at $-40{ }^{\circ} \mathrm{C}$. The mixture was warmed to room temperature and stirred for $8 \mathrm{~h}$. The reaction was quenched with $\mathrm{H}_{2} \mathrm{O}$ at $0{ }^{\circ} \mathrm{C}$. The mixture was filtered through a Cerite pad, and the filtrate was washed with brine, dried $\left(\mathrm{Na}_{2} \mathrm{SO}_{4}\right)$, filtered, and concentrated in vacuo. The residue was purified by silica gel column chromatography (2\% EtOAc/hexane) to afford 21 (3.51 g, $12.3 \mathrm{mmol}, 83 \%$ over 2 steps) as a colorless oil. ${ }^{1} \mathrm{H} \mathrm{NMR}\left(\mathrm{CDCl}_{3}, 400\right.$ MHz) $\delta 5.79(\mathrm{~m}, 1 \mathrm{H}, \mathrm{H}-5), 5.05\left(\mathrm{~d}, 1 \mathrm{H}, \mathrm{H}-6 \mathrm{~b}, J_{6 \mathrm{~b}, 5}=17.0 \mathrm{~Hz}\right), 5.03\left(\mathrm{~d}, 1 \mathrm{H}, \mathrm{H}-6 \mathrm{a}, J_{6 \mathrm{a}, 5}=10.6 \mathrm{~Hz}\right), 3.90(\mathrm{~m}, 1 \mathrm{H}, \mathrm{H}-$ 3), 3.64 (m, 3H, H-1, H-2), 2.31 (m, 2H, H-4), 1.34 (s, 3H, H-OC(CH3) $\left.)_{2} \mathrm{O}\right), 1.31$ (s, 3H, H-OC(CH3) $\left.2 \mathrm{O}\right), 0.83(\mathrm{~s}, 9 \mathrm{H}$, $\left.\mathrm{H}-{ }^{t} \mathrm{Bu}\right), 0.00\left(\mathrm{~s}, 6 \mathrm{H}, \mathrm{H}-\mathrm{SiCH}_{3}\right) ;{ }^{13} \mathrm{C} \mathrm{NMR}\left(\mathrm{CDCl}_{3}, 100 \mathrm{MHz}\right) \delta 134.3,117.5,109.2,108.7,80.9,78.8,64.0,63.7$, 37.7, 27.4, 27.2, 27.1, 26.1, 18.5, -5.2; ESIMS-LR m/z 309 [(M+Na) ${ }^{+}$; HRMS (ESI) m/z: [(M+H) $\left.{ }^{+}\right] \mathrm{Calcd}$ for $\mathrm{C}_{15} \mathrm{H}_{31} \mathrm{O}_{3} \mathrm{Si}$ 287.2042; Found 287.2043; $[\alpha]^{20}{ }_{\mathrm{D}}-6.7\left(c 0.95, \mathrm{CHCl}_{3}\right)$. This is known compound as its enantiomer. ${ }^{\mathrm{S} 4}$

\section{(S)-4-Benzyl-3-\{(2S,3R)-4-[(4S,5S)-5-tert-butyldimethylsilyloxymethyl-2,2-dimethyl-1,3-dioxolan-4-yl]-3-} hydroxy-2-methylbutanoyl\}oxazolidin-2-one (24)

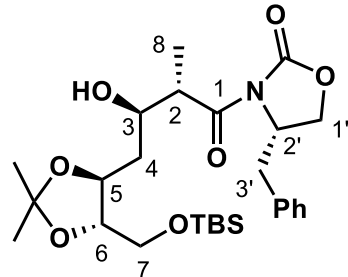

A solution of $21(1.14 \mathrm{~g}, 4.0 \mathrm{mmol})$ in dioxane $(30 \mathrm{~mL})$ and $\mathrm{H}_{2} \mathrm{O}(10 \mathrm{~mL})$ was treated with 2,6-lutidine (927 $\mu \mathrm{L}, 8.0 \mathrm{mmol}), \mathrm{K}_{2} \mathrm{OsO}_{4} \cdot 2 \mathrm{H}_{2} \mathrm{O}(29 \mathrm{mg}, 0.08 \mathrm{mmol})$ and $\mathrm{NaIO}_{4}(3.4 \mathrm{~g}$ $16 \mathrm{mmol}$ ) at $0{ }^{\circ} \mathrm{C}$. The mixture was warmed to room temperture and stirred for $3 \mathrm{~h}$. The mixture was partitioned between $\mathrm{CH}_{2} \mathrm{Cl}_{2}$ and $\mathrm{H}_{2} \mathrm{O}$. The organic phase was washed with $1 \mathrm{M}$ 
aq. $\mathrm{HCl}$, sat. aq. $\mathrm{NaHCO}_{3}, \mathrm{H}_{2} \mathrm{O}$ and brine, dried $\left(\mathrm{Na}_{2} \mathrm{SO}_{4}\right)$, filtered and concentrated in vacuo. The residue was purified by silica gel column chromatography $(\phi 4 \times 10 \mathrm{~cm}, 5 \rightarrow 10 \%$ EtOAc/hexane) to afford a crude aldehyde 22 as a colorless oil. A solution of $23(1.25 \mathrm{~g}, 5.36 \mathrm{mmol})$ in $\mathrm{CH}_{2} \mathrm{Cl}_{2}(30 \mathrm{~mL})$ was treated with $\mathrm{TiCl}_{4}(588 \mu \mathrm{L}, 5.36$ mmol) at $0{ }^{\circ} \mathrm{C}$ for $15 \mathrm{~min} . N, N$-Diisopropylethylamine $(934 \mu \mathrm{L}, 5.36 \mathrm{mmol})$ was added to the mixture, which was stirred for $20 \mathrm{~min}$. $N$-Methylpyrrolidinone $(506 \mu \mathrm{L}, 5.36 \mathrm{mmol})$ was added to the mixture, which was further stirred for $10 \mathrm{~min}$. A solution of $22(1.03 \mathrm{~g})$ in $\mathrm{CH}_{2} \mathrm{Cl}_{2}(6 \mathrm{~mL})$ was added to the mixture via cannula, and the whole mixture was stirred for $2 \mathrm{~h}$. The reaction was quenched with sat. aq. $\mathrm{NH}_{4} \mathrm{Cl}$. The organic phase was washed with $1 \mathrm{M} a q$. $\mathrm{HCl}$, sat. aq. $\mathrm{NaHCO}_{3}, \mathrm{H}_{2} \mathrm{O}$ and brine, dried $\left(\mathrm{Na}_{2} \mathrm{SO}_{4}\right)$, filtered and concentrated in vacuo. The residue was purified by flash silica gel column chromatography $(\phi 4 \times 12 \mathrm{~cm}, 10 \rightarrow 30 \%$ EtOAc/hexane) to afford 24 (2.00 g, $3.84 \mathrm{mmol}$, $96 \%$ over 2 steps) as a colorless oil. ${ }^{1} \mathrm{H}$ NMR $\left(\mathrm{CDCl}_{3}, 400 \mathrm{MHz}\right) \delta 7.30(\mathrm{~m}, 5 \mathrm{H}, \mathrm{H}-\mathrm{Ph}), 4.68\left(\mathrm{ddd}, 1 \mathrm{H}, \mathrm{H}-2^{\prime}, J_{2^{\prime}, 1^{\prime}}=\right.$ $\left.10.1, J_{2^{\prime}, 3^{\prime}}=3.6, J_{2^{\prime}, 3^{\prime}}=6.9 \mathrm{~Hz}\right), 4.20\left(\mathrm{~m}, 2 \mathrm{H}, \mathrm{H}-1^{\prime}\right), 4.18(\mathrm{~m}, 1 \mathrm{H}, \mathrm{H}-3), 4.04\left(\mathrm{dt}, 1 \mathrm{H}, \mathrm{H}-5, J_{5,6}=6.7, J_{5,4}=3.0 \mathrm{~Hz}\right)$, $3.90\left(\mathrm{dq}, 1 \mathrm{H}, \mathrm{H}-2, J_{2,8}=7.3, J_{2,3}=2.3 \mathrm{~Hz}\right), 3.81\left(\mathrm{dd}, 1 \mathrm{H}, \mathrm{H}-7, J_{g e m}=12.2, J_{7,6}=4.2 \mathrm{~Hz}\right), 3.74(\mathrm{~m}, 1 \mathrm{H}, \mathrm{H}-6), 3.67$ $\left(\mathrm{dd}, 1 \mathrm{H}, \mathrm{H}-7, J_{g e m}=10.5, J_{7,6}=6.0 \mathrm{~Hz}\right), 3.44(\mathrm{~s}, 1 \mathrm{H}, \mathrm{H}-\mathrm{OH}), 3.28\left(\mathrm{dd}, 1 \mathrm{H}, \mathrm{H}-3^{\prime}, J_{g e m}=12.2, J_{3^{\prime}, 2^{\prime}}=3.2 \mathrm{~Hz}\right), 2.76(\mathrm{dd}$, $\left.1 \mathrm{H}, \mathrm{H}-3^{\prime}, J_{\text {gem }}=13.8, J_{3^{\prime}, 2^{\prime}}=9.6 \mathrm{~Hz}\right), 1.88\left(\mathrm{dt}, 1 \mathrm{H}, \mathrm{H}-4, J_{\text {gem }}=14.6, J_{4,5}=3.2 \mathrm{~Hz}\right), 1.78\left(\mathrm{dt}, 1 \mathrm{H}, \mathrm{H}-4, J_{\text {gem }}=14.6, J_{4,3}\right.$ $=9.2 \mathrm{~Hz}), 1.39\left(\mathrm{~s}, 3 \mathrm{H}, \mathrm{H}-\mathrm{OC}\left(\mathrm{CH}_{3}\right)_{2} \mathrm{O}\right), 1.37\left(\mathrm{~s}, 3 \mathrm{H}, \mathrm{H}-\mathrm{OC}\left(\mathrm{CH}_{3}\right)_{2} \mathrm{O}\right), 1.28\left(\mathrm{~d}, 3 \mathrm{H}, \mathrm{H}-8, J_{8,2}=7.3 \mathrm{~Hz}\right), 0.89(\mathrm{~s}, 9 \mathrm{H}, \mathrm{H}-$ $\left.{ }^{t} \mathrm{Bu}\right), 0.07$ (s, $\left.6 \mathrm{H}, \mathrm{H}-\mathrm{SiCH}_{3}\right) ;{ }^{13} \mathrm{C} \mathrm{NMR}\left(\mathrm{CDCl}_{3}, 100 \mathrm{MHz}\right) \delta$ 176.0, 132.2, 152.0, 135.4, 129.6, 129.1, 127.5, 109.2, $81.3,78.5,71.1,66.2,63.7,55.5,42.7,38.0,37.5,27.4,27.0,26.0,18.5,11.9,-5.2 ;$ ESIMS-LR $m / z 544\left[(\mathrm{M}+\mathrm{Na})^{+}\right]$; HRMS (ESI) m/z: [(M+Na) $\left.{ }^{+}\right]$Calcd for $\mathrm{C}_{27} \mathrm{H}_{43} \mathrm{NO}_{7} \mathrm{NaSi} 544.2706$; Found 544.2726; $[\alpha]^{20}{ }_{\mathrm{D}}+38.0\left(c\right.$ 1.00, $\left.\mathrm{CHCl}_{3}\right)$.

\section{(S)-4-Benzyl-3-\{(2S,3R)-4-[(4S,5S)-5-tert-butyldimethylsilyloxymethyl-2,2-dimethyl-1,3-dioxolan-4-yl]-3-}

\section{methoxymethoxy-2-methylbutanoyl\}oxazolidin-2-one (25)}

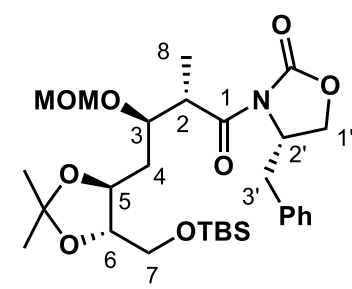

A solution of $24(1.02 \mathrm{~g}, 1.96 \mathrm{mmol})$ in $\mathrm{CH}_{2} \mathrm{Cl}_{2}(10 \mathrm{~mL})$ was treated with ${ }^{i} \operatorname{Pr}_{2} \mathrm{NEt}(1.36$ $\mathrm{g}, 7.84 \mathrm{mmol})$ and $\mathrm{MOMCl}(2.98 \mathrm{~mL}, 3.92 \mathrm{mmol})$ at $0{ }^{\circ} \mathrm{C}$. The mixture was warmed to room temperature and stirred for $18 \mathrm{~h} . N, N$-Diisopropylethylamine $(0.65 \mathrm{~g}, 3.92 \mathrm{mmol})$ and $\mathrm{MOMCl}(150 \mu \mathrm{L}, 1.96 \mathrm{mmol})$ were added to the mixture, which was stirred for $4 \mathrm{~h}$. The reaction was quenched with $\mathrm{H}_{2} \mathrm{O}$. The mixture was partitioned between EtOAc and $\mathrm{H}_{2} \mathrm{O}$. The organic phase was washed with $1 \mathrm{M}$ aq. $\mathrm{HCl}$, sat. aq. $\mathrm{NaHCO}_{3}, \mathrm{H}_{2} \mathrm{O}$ and brine, dried $\left(\mathrm{Na}_{2} \mathrm{SO}_{4}\right)$, filtered and concentrated in vacuo. The residue was purified by silica gel column chromatography $(\phi 5 \times 13 \mathrm{~cm}, 13 \%$ EtOAc/hexane) to afford $25(1.10 \mathrm{~g}, 1.94 \mathrm{mmol}, 99 \%)$ as a colorless oil. ${ }^{1} \mathrm{H} \mathrm{NMR}\left(\mathrm{CDCl}_{3}, 400 \mathrm{MHz}\right) \delta 7.26(\mathrm{~m}, 5 \mathrm{H}$, $\mathrm{H}-\mathrm{Ph}), 4.70\left(\mathrm{~d}, 1 \mathrm{H}, \mathrm{H}-\mathrm{OCH} \mathrm{H}_{2} \mathrm{O}, J_{g e m}=7.3 \mathrm{~Hz}\right), 4.65\left(\mathrm{~m}, 1 \mathrm{H}, \mathrm{H}-2^{\prime}\right), 4.62\left(\mathrm{~d}, 1 \mathrm{H}, \mathrm{H}-\mathrm{OCH} \mathrm{H}_{2} \mathrm{O}, J_{g e m}=7.3 \mathrm{~Hz}\right), 4.15(\mathrm{~d}$, $\left.2 \mathrm{H}, \mathrm{H}-1^{\prime}, J_{\text {gem }}=5.0 \mathrm{~Hz}\right), 4.09(\mathrm{~m}, 2 \mathrm{H}, \mathrm{H}-2, \mathrm{H}-3), 4.00\left(\mathrm{dt}, 1 \mathrm{H}, \mathrm{H}-5, J_{5,4}=4.1, J_{5,6}=8.5 \mathrm{~Hz}\right), 3.75\left(\mathrm{dd}, 1 \mathrm{H}, \mathrm{H}-7, J_{g e m}\right.$ $\left.=11.4, J_{7,6}=4.6 \mathrm{~Hz}\right), 3.71\left(\mathrm{dd}, 1 \mathrm{H}, \mathrm{H}-7, J_{g e m}=11.4, J_{7,6}=5.0 \mathrm{~Hz}\right), 3.56\left(\mathrm{dt}, 1 \mathrm{H}, \mathrm{H}-6, J_{6,5}=8.5, J_{6,7}=4.6 \mathrm{~Hz}\right), 3.37$ $\left(\mathrm{s}, 3 \mathrm{H}, \mathrm{H}-\mathrm{OCH}_{2} \mathrm{OCH}_{3}\right), 3.25\left(\mathrm{dd}, 1 \mathrm{H}, \mathrm{H}-1^{\prime}, J_{g e m}=13.8, J_{1^{\prime}, 2^{\prime}}=3.2 \mathrm{~Hz}\right), 2.78\left(\mathrm{dd}, 1 \mathrm{H}, \mathrm{H}-1^{\prime}, J_{g e m}=13.8, J_{1^{\prime}, 2^{\prime}}=10.1\right.$ 
Hz), $2.04(\mathrm{~m}, 1 \mathrm{H}, \mathrm{H}-4), 1.86\left(\mathrm{ddd}, 1 \mathrm{H}, \mathrm{H}-4, J_{g e m}=13.8, J_{4,3}=9.6, J_{4,5}=4.1 \mathrm{~Hz}\right), 1.31\left(\mathrm{~m}, 9 \mathrm{H}, \mathrm{H}-8, \mathrm{H}-\mathrm{OC}(\mathrm{CH})_{2} \mathrm{O}\right)$,

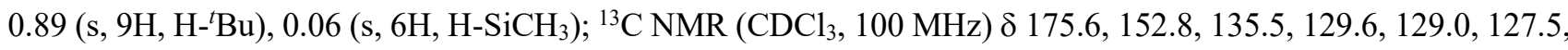
108.5, 96.3, 81.5, 74.7, 66.0, 63.3, 56.1, 55.3, 41.5, 38.2, 34.6, 27.4, 26.7, 26.0, 18.5, 14.7, -5.2; ESIMS-LR $m / z$ 588 $\left[(\mathrm{M}+\mathrm{Na})^{+}\right]$; HRMS (ESI) m/z: $\left[(\mathrm{M}+\mathrm{Na})^{+}\right]$Calcd for $\mathrm{C}_{29} \mathrm{H}_{47} \mathrm{NO}_{8} \mathrm{NaSi} 588.2969$; Found 588.2966; $[\alpha]^{20}{ }_{\mathrm{D}}+20.3(c 0.76$, $\left.\mathrm{CHCl}_{3}\right)$.

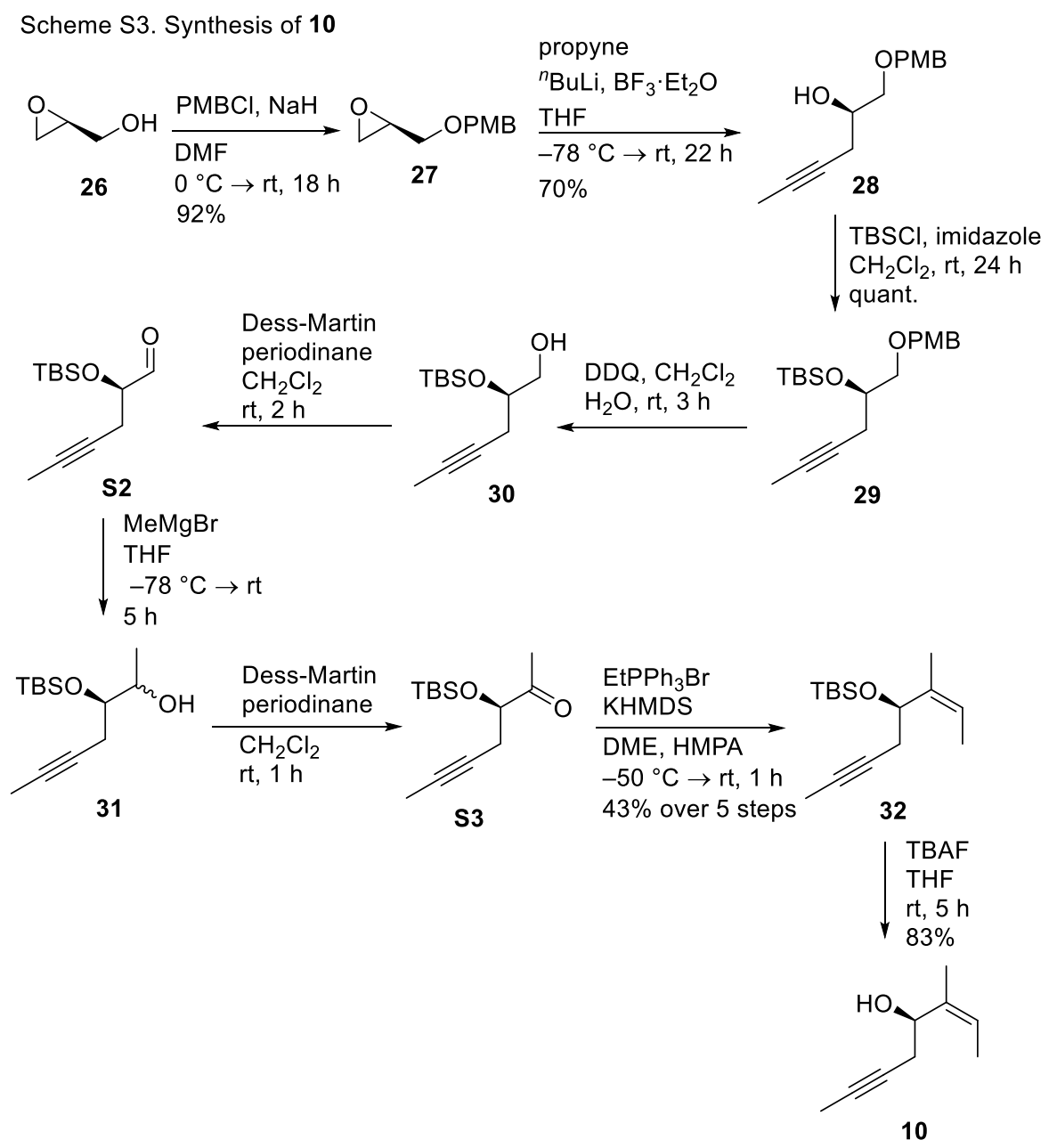

\section{(R)-2-[(4-Methoxybenzyloxy)methyl]oxirane (27)}

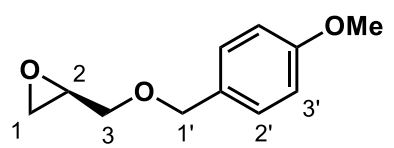

A solution of 26 (5.0 g, $67.5 \mathrm{mmol})$ in DMF (135 mL) was treated with PMBCl (10.1 $\mathrm{mL}, 74.2 \mathrm{mmol})$ and $\mathrm{NaH}(2.96 \mathrm{~g}, 74.2 \mathrm{mmol})$ at $0{ }^{\circ} \mathrm{C}$. The mixture was warmed to room temperature and stirred for $18 \mathrm{~h}$. The reaction was quenched with sat. aq. $\mathrm{NH}_{4} \mathrm{Cl}$. The mixture was partitioned between EtOAc and $\mathrm{H}_{2} \mathrm{O}$. The organic phase was washed with sat. aq. $\mathrm{NaHCO}_{3}, \mathrm{H}_{2} \mathrm{O}$ and brine, dried $\left(\mathrm{Na}_{2} \mathrm{SO}_{4}\right)$, filtered and concentrated in vacuo. The residue was purified by silica gel column chromatography $\left(\phi 10 \times 15 \mathrm{~cm}, 25 \%\right.$ EtOAc/hexane) to afford $27(12.0 \mathrm{~g}, 61.8 \mathrm{mmol}, 92 \%)$ as a colorless oil. ${ }^{1} \mathrm{H}$ $\operatorname{NMR}\left(\mathrm{CDCl}_{3}, 400 \mathrm{MHz}\right) \delta 7.27$ (d, 2H, H-2', $\left.J_{2^{\prime}, 3^{\prime}}=7.8 \mathrm{~Hz}\right), 6.89$ (d, 2H, H-3', $\left.J_{3^{\prime}, 2^{\prime}}=7.8 \mathrm{~Hz}\right), 4.57$ (d, 1H, H-1', $J_{g e m}$ $=11.9 \mathrm{~Hz}), 4.48\left(\mathrm{~d}, 1 \mathrm{H}, \mathrm{H}-1^{\prime}, J_{g e m}=11.9 \mathrm{~Hz}\right), 3.80\left(\mathrm{~s}, 3 \mathrm{H}, \mathrm{H}-\mathrm{C}_{6} \mathrm{H}_{4} \mathrm{OCH}_{3}\right), 3.73\left(\mathrm{dd}, 1 \mathrm{H}, \mathrm{H}-1, J_{\text {gem }}=11.9, J_{1,2}=3.2\right.$ $\mathrm{Hz}), 3.41\left(\mathrm{dd}, 1 \mathrm{H}, \mathrm{H}-1, J_{\text {gem }}=11.9, J_{1,2}=6.2 \mathrm{~Hz}\right), 3.17(\mathrm{~m}, 1 \mathrm{H}, \mathrm{H}-2), 2.78\left(\mathrm{dd}, 1 \mathrm{H}, \mathrm{H}-3, J_{3,2}=J_{\text {gem }}=4.6 \mathrm{~Hz}\right), 2.60$ 
$\left(\mathrm{dd}, 1 \mathrm{H}, \mathrm{H}-3, J_{3,2}=2.7, J_{\text {gem }}=5.0 \mathrm{~Hz}\right)$; ESIMS-LR $m / z 217\left[(\mathrm{M}+\mathrm{Na})^{+}\right]$. This is a known compound. ${ }^{\mathrm{S} 5}$

\section{(R)-1-(4-Methoxybenzyloxy)hex-4-yn-2-ol (28)}

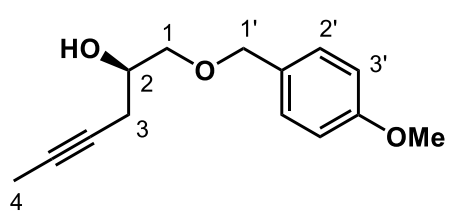

A solution of propyne $(19.4 \mathrm{~mL}, 7.71 \mathrm{mmol}, 3 \%$ in heptane) was treated with ${ }^{n} \operatorname{BuLi}\left(4.97 \mathrm{~mL}, 7.71 \mathrm{mmol}, 1.55 \mathrm{M}\right.$ in hexane) at $-78^{\circ} \mathrm{C}$ for $1.5 \mathrm{~h}$. A solution of 27 (500 mg, $2.57 \mathrm{mmol})$ and $\mathrm{BF}_{3} \cdot \mathrm{OEt}_{2}(0.65 \mathrm{~mL}, 5.14 \mathrm{mmol})$ in THF $(8.6 \mathrm{~mL})$ was added to the mixture, which was warmed to room temperature and stirred for $22 \mathrm{~h}$.

The reaction was quenched with sat. aq. $\mathrm{NaHCO}_{3}$. The mixture was concentrated in vacuo, and the residue was partitioned between EtOAc and $\mathrm{H}_{2} \mathrm{O}$. The organic phase was washed with $\mathrm{H}_{2} \mathrm{O}$ and brine, dried $\left(\mathrm{Na}_{2} \mathrm{SO}_{4}\right)$, filtered and concentrated in vacuo. The residue was purified by hi-flash silica gel column chromatography $(\phi 2 \times 6.5 \mathrm{~cm}$, $10 \rightarrow 20 \%$ EtOAc/hexane) to afford 28 ( $273 \mathrm{mg}, 1.17 \mathrm{mmol}, 46 \%, 70 \% \mathrm{brsm})$ and 27 (175 $\mathrm{mg}, 0.90 \mathrm{mmol}, 35 \%$ ) as

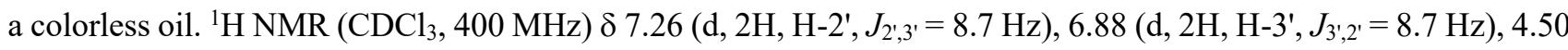
(s, 2H, H-1'), 3.91 (ddt, 1H, H-2, $J_{2,3}=10.1, J_{2,1}=6.6, J_{2,1}=3.7 \mathrm{~Hz}$ ), 3.81 (s, 3H, H-C $\left.\mathrm{C}_{4} \mathrm{OCH} H_{3}\right), 3.56$ (dd, 1H, H-1, $\left.J_{\text {gem }}=9.4, J_{1,2}=3.7 \mathrm{~Hz}\right), 3.45\left(\mathrm{dd}, 1 \mathrm{H}, \mathrm{H}-1, J_{\text {gem }}=9.4, J_{1,2}=6.6 \mathrm{~Hz}\right), 2.37$ (m, 2H, H-3), 2.50 (br s, 1H, H-OH), 1.78 (t, 3H, H-4, $\left.J_{4,3}=2.7 \mathrm{~Hz}\right) ;{ }^{13} \mathrm{C} \mathrm{NMR}\left(\mathrm{CDCl}_{3}, 100 \mathrm{MHz}\right) \delta 159.4,130.2,129.5,114.0,74.8,73.2,72.9,69.2,55.4$, 24.0, 3.7; ESIMS-LR $m / z 257$ [(M+Na) ${ }^{+}$; HRMS (ESI) m/z: [(M+Na) $)^{+}$Calcd for $\mathrm{C}_{14} \mathrm{H}_{18} \mathrm{O}_{3} \mathrm{Na}$ 257.1154; Found 257.1167; $[\alpha]^{20}{ }_{\mathrm{D}}-9.8\left(c 1.10, \mathrm{CHCl}_{3}\right)$.

\section{(R)-2-tert-Butyldimethylsilyloxy-1-(4-methoxybenzyloxy)hex-4-yne (29)}

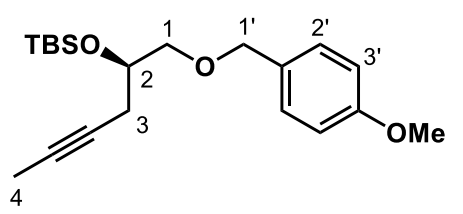

A solution of $\mathbf{2 8}(4.1 \mathrm{~g}, 17.5 \mathrm{mmol})$ in DMF $(100 \mathrm{~mL})$ was treated with imidazole $(2.86 \mathrm{~g}, 42 \mathrm{mmol})$ and $\operatorname{TBSCl}(3.17 \mathrm{~g}, 21 \mathrm{mmol})$ at room temperature for $24 \mathrm{~h}$. The reaction was quenched with $\mathrm{MeOH}$. The mixture was partitioned between EtOAc and $\mathrm{H}_{2} \mathrm{O}$. The organic phase was washed with $1 \mathrm{M}$ aq. $\mathrm{HCl}$, sat. aq. $\mathrm{NaHCO}_{3}, \mathrm{H}_{2} \mathrm{O}$ and brine, dried $\left(\mathrm{Na}_{2} \mathrm{SO}_{4}\right)$, filtered and concentrated in vacuo. The residue was purified by silica gel column chromatography $\left(\phi 6 \times 15 \mathrm{~cm}, 5 \% \mathrm{E}_{2} \mathrm{O} /\right.$ hexane $)$ to afford $29\left(6.1 \mathrm{~g}, 17.5 \mathrm{mmol}\right.$, quant.) as a colorless oil. ${ }^{1} \mathrm{H}$ NMR $\left(\mathrm{CDCl}_{3}, 400 \mathrm{MHz}\right) \delta 7.24$ (m, 2H, H-2'), 6.87 (d, 2H, H-3', $\left.J_{3^{\prime}, 2^{\prime}}=8.7 \mathrm{~Hz}\right), 4.47$ (s, 2H, H-1'), 3.90 (ddt, 1H, H-2, $J_{2,3}$ $\left.=11.9, J_{2,1}=5.5, J_{2,1}=5.0 \mathrm{~Hz}\right), 3.80\left(\mathrm{~s}, 3 \mathrm{H}, \mathrm{H}-\mathrm{C}_{6} \mathrm{H}_{4} \mathrm{OCH} \mathrm{H}_{3}\right), 3.47\left(\mathrm{dd}, 1 \mathrm{H}, \mathrm{H}-1, J_{\text {gem }}=10.1, J_{1,2}=5.0 \mathrm{~Hz}\right), 3.41(\mathrm{dd}$, $\left.1 \mathrm{H}, \mathrm{H}-1, J_{\text {gem }}=10.1, J_{1,2}=5.5 \mathrm{~Hz}\right), 2.39(\mathrm{~m}, 1 \mathrm{H}, \mathrm{H}-3), 2.28(\mathrm{~m}, 1 \mathrm{H}, \mathrm{H}-3), 1.75\left(\mathrm{t}, 3 \mathrm{H}, \mathrm{H}-4, J_{4,3}=2.8 \mathrm{~Hz}\right), 0.89$ (s, 9H, $\left.\mathrm{H}-{ }^{t} \mathrm{Bu}\right), 0.09$ (s, 3H, H-SiCH 3$), 0.06$ (s, 3H, $\left.\mathrm{H}-\mathrm{SiCH}_{3}\right) ;{ }^{13} \mathrm{C} \mathrm{NMR}\left(\mathrm{CDCl}_{3}, 100 \mathrm{MHz}\right) \delta 159.3,130.7,129.3,113.8$, 73.4, 73.1, 71.1, 55.4, 26.0, 25.1, 18.3, 6.61, 3.7, -4.4; ESIMS-LR m/z 371 [(M+Na) $\left.{ }^{+}\right]$; HRMS (ESI) m/z: [(M+Na) $\left.{ }^{+}\right]$ Calcd for $\mathrm{C}_{20} \mathrm{H}_{32} \mathrm{O}_{3} \mathrm{NaSi} 371.2018$; Found 371.2002; $[\alpha]^{20}{ }_{\mathrm{D}}-2.8\left(c 0.70, \mathrm{CHCl}_{3}\right)$. 


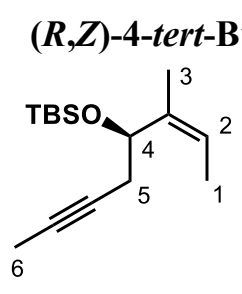

A solution of $29(763 \mathrm{mg}, 2.19 \mathrm{mmol})$ in $\mathrm{CH}_{2} \mathrm{Cl}_{2}(18 \mathrm{~mL})$ and phosphate buffer $(1 \mathrm{~mL}, \mathrm{pH} 7)$ was treated with DDQ (994 mg, $4.3 \mathrm{mmol}$ ) at room temperature for $3 \mathrm{~h}$. The mixture was filtered through a Cerite pad. The filtrate was washed with sat. aq. $\mathrm{NaHCO}_{3}, \mathrm{H}_{2} \mathrm{O}$, brine, dried $\left(\mathrm{Na}_{2} \mathrm{SO}_{4}\right)$, filtered and concentrated in vacuo to afford 30. A solution of the residue (467 mg) in $\mathrm{CH}_{2} \mathrm{Cl}_{2}$ was treated with Dess-Martin periodinane $(1.0 \mathrm{~g}, 2.4 \mathrm{mmol})$ at room temperature for $2 \mathrm{~h}$. The reaction was quenched with sat. aq. $\mathrm{Na}_{2} \mathrm{~S}_{2} \mathrm{O}_{3}$ and sat. aq. $\mathrm{NaHCO}_{3}$. The mixture was partitioned between EtOAc and $\mathrm{H}_{2} \mathrm{O}$. The organic phase was washed with brine, dried $\left(\mathrm{Na}_{2} \mathrm{SO}_{4}\right)$, filtered and concentrated in vacuo to give $\mathbf{S 2}$. A solution of the residue (500 $\mathrm{mg})$ in THF $(20 \mathrm{~mL})$ was treated with $\mathrm{MeLi}\left(2.0 \mathrm{~mL}, 3.0 \mathrm{mmol}, 1.5 \mathrm{M}\right.$ in $\left.\mathrm{Et}_{2} \mathrm{O}\right)$ at $-78^{\circ} \mathrm{C}$. The mixture was warmed to room temperature and stirred for $5 \mathrm{~h}$. The reaction was quenched with sat. aq. $\mathrm{NH}_{4} \mathrm{Cl}$. The mixture was partitioned between EtOAc and $\mathrm{H}_{2} \mathrm{O}$. The organic phase was washed with $1 \mathrm{M}$ aq. $\mathrm{HCl}$, sat. aq. $\mathrm{NaHCO}_{3}, \mathrm{H}_{2} \mathrm{O}$ and brine, dried $\left(\mathrm{Na}_{2} \mathrm{SO}_{4}\right)$, filtered and concentrated in vacuo to give 31. A solution of the residue (319 $\left.\mathrm{mg}, 1.31 \mathrm{mmol}\right)$ in $\mathrm{CH}_{2} \mathrm{Cl}_{2}$ $(13 \mathrm{~mL})$ was treated with Dess-Martin periodinane $(667 \mathrm{mg}, 1.57 \mathrm{mmol})$ at room temperature for $1 \mathrm{~h}$. The reaction was quenched with sat. aq. $\mathrm{Na}_{2} \mathrm{~S}_{2} \mathrm{O}_{3}$ and sat. aq. $\mathrm{NaHCO}_{3}$. The mixture was partitioned between EtOAc and $\mathrm{H}_{2} \mathrm{O}$. The organic phase was washed with brine, dried $\left(\mathrm{Na}_{2} \mathrm{SO}_{4}\right)$, filtered and concentrated in vacuo to afford a crude $\mathbf{S 3}$ (256 mg). Ethyltriphenylphosphonium bromide (2.37 g, $6.39 \mathrm{mmol})$ was dissolved in DME (10 mL) and HMPA (1 $\mathrm{mL})$. The mixture was treated with KHMDS $(10.6 \mathrm{~mL}, 5.3 \mathrm{mmol}, 0.5 \mathrm{M}$ in toluene) at room temperature for $10 \mathrm{~min}$, and cooled to $-50^{\circ} \mathrm{C}$. A solution of the crude $\mathbf{S 3}(256 \mathrm{mg})$ in DME $(1 \mathrm{~mL})$ was added to the mixture, which was warmed to room temperature and stirred for $1 \mathrm{~h}$. The reaction was quenched with phosphate buffer (pH 7.0). The mixture was partitioned between $\mathrm{Et}_{2} \mathrm{O}$ and $\mathrm{H}_{2} \mathrm{O}$. The organic phase was washed with brine, dried $\left(\mathrm{Na}_{2} \mathrm{SO}_{4}\right)$, filtered and concentrated in vacuo. The residue was purified by silica gel column chromatography $(\phi 2.5 \times 12 \mathrm{~cm}, 5 \%$ $\mathrm{Et}_{2} \mathrm{O} /$ hexane) to afford $32(238 \mathrm{mg}, 0.94 \mathrm{mmol}, 43 \%$ over 5 steps $)$ as a colorless oil. ${ }^{1} \mathrm{H}$ NMR $\left(\mathrm{CDCl}_{3}, 400 \mathrm{MHz}\right) \delta$ 5.25 (q, $\left.1 \mathrm{H}, \mathrm{H}-2, J_{2,1}=6.4 \mathrm{~Hz}\right), 4.69$ (t, $\left.1 \mathrm{H}, \mathrm{H}-4, J_{4,5}=6.9 \mathrm{~Hz}\right), 2.35\left(\mathrm{ddq}, 1 \mathrm{H}, \mathrm{H}-5, J_{g e m}=13.0, J_{5,4}=6.0, J_{5,6}=2.3\right.$ Hz), 2.21 (ddq, 1H, H-5, $J_{g e m}=13.0, J_{5,4}=4.1, J_{5,6}=2.3 \mathrm{~Hz}$ ), 1.75 (t, 3H, H-6, $J_{6,5}=2.3 \mathrm{~Hz}$ ), 1.63 (s, 3H, H-3), 1.61

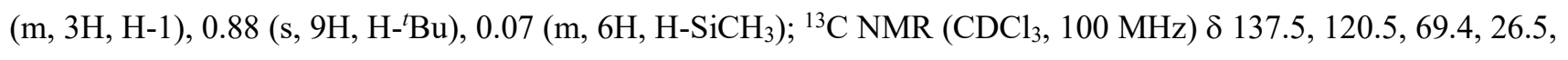
$25.9,18.4,17.4,13.2,3.6,-4.8 ;[\alpha]^{20} \mathrm{D}-2.6\left(c 0.90, \mathrm{CHCl}_{3}\right)$. This is a known compound. ${ }^{\mathrm{S} 6}$

\section{(R,Z)-3-Methyloct-2-en-6-yn-4-ol (10)}

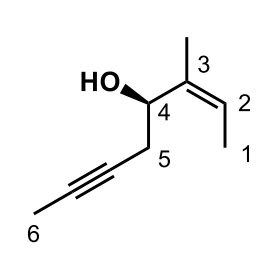

A solution of $32(235 \mathrm{mg}, 0.93 \mathrm{mmol})$ in THF $(10 \mathrm{~mL})$ was treated with TBAF $(1.4 \mathrm{~mL}, 1.4$ mmol, $1 \mathrm{M}$ in THF) at room temperature for $5 \mathrm{~h}$. The mixture was partitioned between $\mathrm{Et}_{2} \mathrm{O}$ and $\mathrm{H}_{2} \mathrm{O}$. The organic phase was washed with $1 \mathrm{M}$ aq. $\mathrm{HCl}$, sat. aq. $\mathrm{NaHCO}_{3}, \mathrm{H}_{2} \mathrm{O}$ and brine, dried $\left(\mathrm{Na}_{2} \mathrm{SO}_{4}\right)$, filtered and concentrated in vacuo. The residue was purified by flash silica gel column chromatography $\left(\phi 2 \times 10 \mathrm{~cm}, 17 \% \mathrm{Et}_{2} \mathrm{O} /\right.$ hexane $)$ to afford $10(115 \mathrm{mg}, 0.83 \mathrm{mmol}, 89 \%)$ as a colorless oil. ${ }^{1} \mathrm{H} \mathrm{NMR}$ 
$\left(\mathrm{CDCl}_{3}, 400 \mathrm{MHz}\right) \delta 5.35\left(\mathrm{q}, 1 \mathrm{H}, \mathrm{H}-2, J_{2,1}=6.9 \mathrm{~Hz}\right), 4.70\left(\mathrm{ddd}, 1 \mathrm{H}, \mathrm{H}-4, J_{4,5}=8.7, J_{4,5}=5.5, J_{4,5}=2.3 \mathrm{~Hz}\right), 2.46$ $\left(\mathrm{ddq}, 1 \mathrm{H}, \mathrm{H}-5, J_{\text {gem }}=16.5, J_{5,4}=8.7, J_{5,6}=2.3 \mathrm{~Hz}\right), 2.27\left(\mathrm{ddq}, 1 \mathrm{H}, \mathrm{H}-5, J_{g e m}=16.5, J_{5,4}=5.5, J_{5,6}=2.8 \mathrm{~Hz}\right), 1.98(\mathrm{~d}$, $\left.1 \mathrm{H}, \mathrm{H}-\mathrm{OH}, J_{\mathrm{OH}, 4}=2.3 \mathrm{~Hz}\right), 1.80\left(\mathrm{dd}, 1 \mathrm{H}, \mathrm{H}-6, J_{6,5}=2.8, J_{6,5}=2.3 \mathrm{~Hz}\right), 1.69(\mathrm{~s}, 3 \mathrm{H}, \mathrm{H}-3), 1.61$ (d, 3H, H-1, $J_{1,2}=6.9$ $\mathrm{Hz}) ;{ }^{13} \mathrm{C} \mathrm{NMR}\left(\mathrm{CDCl}_{3}, 100 \mathrm{MHz}\right) \delta 136.1,122.5,78.1,75.7,68.3,25.9,17.5,13.1,3.7 ;[\alpha]^{20}{ }_{\mathrm{D}}+8.6\left(c 0.90, \mathrm{CHCl}_{3}\right)$. This is a known compound. ${ }^{\mathrm{S} 6}$

Scheme S3. Synthesis of $\mathbf{3 5}$

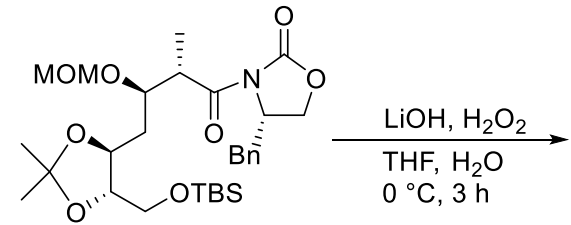

25

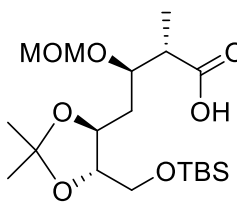

9
1) 10, EDCI, DMAP $\mathrm{CH}_{2} \mathrm{Cl}_{2}, \mathrm{rt}, 22 \mathrm{~h}$

2) $3 \mathrm{HF} \cdot \mathrm{Et}_{3} \mathrm{~N}, \mathrm{THF}, \mathrm{rt}, 26 \mathrm{~h}$ $66 \%$ over 3 steps (from 25)

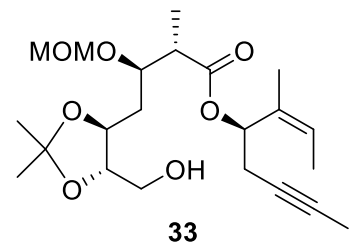

33

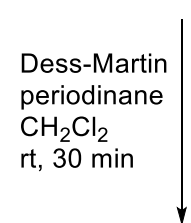<smiles>C/C=C(/C)[C@H](C/C=C(/C)[C@@H](O)[C@H]1OC(C)(C)O[C@@H]1C[C@H](OC)[C@@H](C)OC)OC</smiles>

34

p-nitrobenzoic acid DMEAD, $\mathrm{PhOPPh}_{2}$ toluene, $0^{\circ} \mathrm{C} \rightarrow \mathrm{rt}$ $1 \mathrm{~h}, 67 \%$

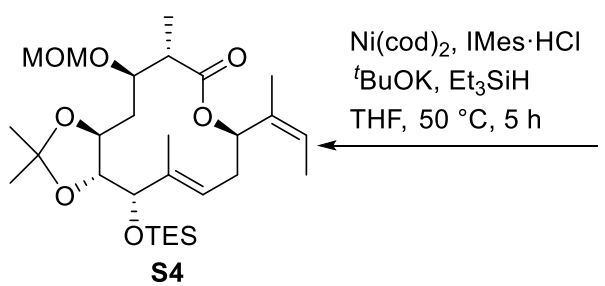

S4

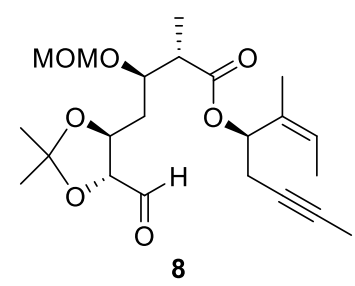

S5

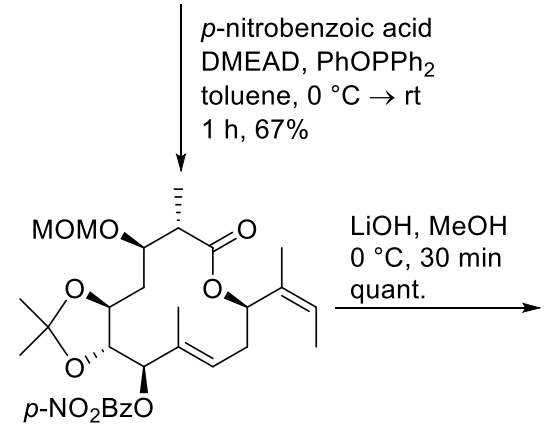

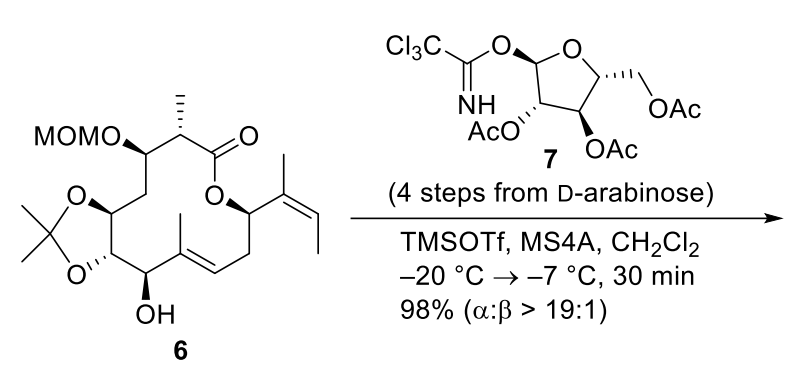

6

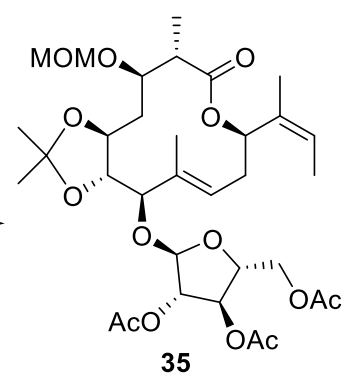

(R,Z)-3-Methyloct-2-en-6-yn-4-yl (2S,3R)-4-[(4S,5S)-5-hydroxymethyl-2,2-dimethyl-1,3-dioxolan-4-yl]-3methoxymethoxy-2-methylbutanoate (33)<smiles>CC#C/C=C(/C)C(OC)OC(=O)C(C)[C@H](CC1OC(C)(C)O[C@H]1CC)OC</smiles>

A solution of $25(1.68 \mathrm{~g}, 2.97 \mathrm{mmol})$ in THF $(24 \mathrm{~mL})$ and $\mathrm{H}_{2} \mathrm{O}(6 \mathrm{~mL})$ was treated with $\mathrm{H}_{2} \mathrm{O}_{2}\left(1.35 \mathrm{~mL}, 11.9 \mathrm{mmol}, 30 \%\right.$ in $\left.\mathrm{H}_{2} \mathrm{O}\right)$ and $\mathrm{LiOH}(248 \mathrm{mg}, 5.9 \mathrm{mmol})$ at $0{ }^{\circ} \mathrm{C}$ for $3 \mathrm{~h}$. The reaction was quenched with sat. aq. $\mathrm{Na}_{2} \mathrm{~S}_{2} \mathrm{O}_{3}$. The mixture was partitioned between $\mathrm{Et}_{2} \mathrm{O}$ and sat. aq. $\mathrm{NaHCO}_{3}$. The organic phase was washed with brine, dried $\left(\mathrm{Na}_{2} \mathrm{SO}_{4}\right)$, filtered and concentrated in vacuo to give 9 . A solution of $\mathbf{9}$ and 10 (387 mg, $2.8 \mathrm{mmol})$ in $\mathrm{CH}_{2} \mathrm{Cl}_{2}(28 \mathrm{~mL})$ was treated with DMAP (34 mg, $\left.0.28 \mathrm{mmol}\right)$ and EDCI $(1.07 \mathrm{~g}, 5.6 \mathrm{mmol})$ at room 
temperature for $22 \mathrm{~h}$. The mixture was partitioned between EtOAc and $\mathrm{H}_{2} \mathrm{O}$. The organic phase was washed with 1 $\mathrm{M}$ aq. $\mathrm{HCl}$, sat. aq. $\mathrm{NaHCO}_{3}, \mathrm{H}_{2} \mathrm{O}$ and brine, dried $\left(\mathrm{Na}_{2} \mathrm{SO}_{4}\right)$ filtered, and concentrated in vacuo to afford a crude ester. A solution of the crude ester in THF $(13 \mathrm{~mL})$ was treated with $3 \mathrm{HF} \cdot \mathrm{Et}_{3} \mathrm{~N}(4.2 \mathrm{~mL}, 25.8 \mathrm{mmol})$ at room temperature for $26 \mathrm{~h}$. The mixture was partitioned between EtOAc and $\mathrm{H}_{2} \mathrm{O}$. The organic phase was washed with 1 $\mathrm{M}$ aq. $\mathrm{HCl}$, sat. aq. $\mathrm{NaHCO}_{3}, \mathrm{H}_{2} \mathrm{O}$ and brine, dried $\left(\mathrm{Na}_{2} \mathrm{SO}_{4}\right)$, filtered and concentrated in vacuo. The residue was purified by hi-flash silica gel column chromatography $(\phi 2.6 \times 10 \mathrm{~cm}, 20 \rightarrow 30 \rightarrow 50 \%$ EtOAc/hexane) to afford 33 (808 mg, $1.96 \mathrm{mmol}, 66 \%$ over 3 steps) as a colorless oil. ${ }^{1} \mathrm{H}$ NMR $\left(\mathrm{CDCl}_{3}, 400 \mathrm{MHz}\right) \delta 5.73\left(\mathrm{t}, 1 \mathrm{H}, \mathrm{H}-4^{\prime}, J_{4^{\prime}, 5^{\prime}}=7.3\right.$ $\mathrm{Hz}), 5.44\left(\mathrm{q}, 1 \mathrm{H}, \mathrm{H}-2^{\prime}, J_{2^{\prime}, 1^{\prime}}=6.9 \mathrm{~Hz}\right), 4.68\left(\mathrm{~d}, 1 \mathrm{H}, \mathrm{H}-\mathrm{OCH} \mathrm{H}_{2} \mathrm{O}, J_{g e m}=7.3 \mathrm{~Hz}\right), 4.65\left(\mathrm{~d}, 1 \mathrm{H}, \mathrm{H}-\mathrm{OCH}_{2} \mathrm{O}, J_{g e m}=7.3 \mathrm{~Hz}\right)$, 4.01 (m, 2H, H-3, H-5), 3.80 (m, 1H, H-7), 3.76 (dt, 1H, H-6, J6,5 = 8.2, J6,7 = 4.1 Hz), 3.65 (m, 1H, H-7), 3.38 (s, $\left.3 \mathrm{H}, \mathrm{H}-\mathrm{OCH}_{2} \mathrm{OCH}_{3}\right), 2.75\left(\mathrm{dq}, 1 \mathrm{H}, \mathrm{H}-2, J_{2,3}=14.2, J_{2,8}=7.1 \mathrm{~Hz}\right), 2.25\left(\mathrm{ddq}, 1 \mathrm{H}, \mathrm{H}-5^{\prime}, J_{\text {gem }}=16.5, J_{5^{\prime}, 4^{\prime}}=6.9, J_{5^{\prime}, 6^{\prime}}=\right.$ $2.8 \mathrm{~Hz}$ ), 2.41 (ddq, 1H, H-5', $J_{\text {gem }}=16.5, J_{5^{\prime}, 4^{\prime}}=7.3, J_{5^{\prime}, 6^{\prime}}=2.8 \mathrm{~Hz}$ ), 2.11 (br s, 1H, H-OH), 1.98 (ddd, 1H, H-4, $J_{g e m}$ $\left.=14.6, J_{4,3}=J_{4,5}=6.4 \mathrm{~Hz}\right), 1.83\left(\mathrm{ddd}, 1 \mathrm{H}, \mathrm{H}-4, J_{\text {gem }}=14.6, J_{4,3}=J_{4,5}=5.0 \mathrm{~Hz}\right), 1.75\left(\mathrm{t}, 3 \mathrm{H}, \mathrm{H}-6^{\prime}, J_{6^{\prime} 5^{\prime}}=2.8 \mathrm{~Hz}\right)$, $1.70\left(\mathrm{~d}, 3 \mathrm{H}, \mathrm{H}-1^{\prime}, J_{1^{\prime}, 2^{\prime}}=6.9 \mathrm{~Hz}\right), 1.66$ (s, 3H, H-3'), 1.40 (s, 3H, H-9), 1.39 (s, 3H, H-OC(CH3) $\left.)_{2} \mathrm{O}\right), 1.19$ (d, 3H, H$\left.8, J_{8,2}=7.1 \mathrm{~Hz}\right) ;{ }^{13} \mathrm{C} \mathrm{NMR}\left(\mathrm{CDCl}_{3}, 100 \mathrm{MHz}\right) \delta 173.5,132.3,124.8,108.8,97.0,81.6,74.5,74.1,70.9,62.0,56.0$, 43.7, 36.2, 27.4, 27.1, 22.9, 17.9, 13.3, 12.2, 3.5; ESIMS-LR $m / z 435$ [(M+Na) $]$; HRMS (ESI) m/z: [(M+Na) $)^{+}$Calcd for $\mathrm{C}_{22} \mathrm{H}_{36} \mathrm{O}_{7} \mathrm{Na}$ 435.2359; Found 435.2375; $[\alpha]^{20}{ }_{D}+20.3\left(c\right.$ 0.76, $\left.\mathrm{CHCl}_{3}\right)$.

\section{( $R, Z)$-3-Methyloct-2-en-6-yn-4-yl (2S,3R)-4-[(4S,5R)-5-formyl-2,2-dimethyl-1,3-dioxolan-4-yl]-3-}

\section{methoxymethoxy-2-methylbutanoate (8)}

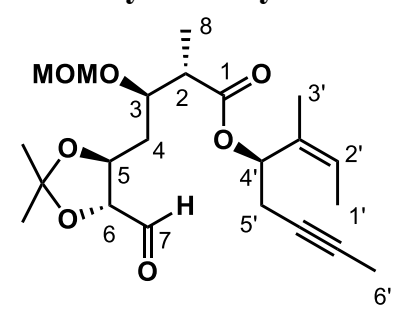

A solution of $33(100 \mathrm{mg}, 0.24 \mathrm{mmol})$ in $\mathrm{CH}_{2} \mathrm{Cl}_{2}(2.4 \mathrm{~mL})$ was treated with DessMartin periodinane $(153 \mathrm{mg}, 0.36 \mathrm{mmol})$ at room temperature for $30 \mathrm{~min}$. The mixture was diluted with hexane, filtered through a Cerite pad, and the filtrate was concentrated in vacuo. The residue was purified by silica gel column chromatography $(40 \%$ EtOAc/hexane) to afford a crude $\mathbf{8}(100 \mathrm{mg})$ as a colorless oil. The crude 8 was used for the next reaction without further purification. ${ }^{1} \mathrm{H}$ NMR $\left(\mathrm{CDCl}_{3}, 400 \mathrm{MHz}\right) \delta 9.72(\mathrm{~s}, 1 \mathrm{H}, \mathrm{CHO}), 5.73(\mathrm{t}, 1 \mathrm{H}, \mathrm{H}-4$ ', $\left.J_{4^{\prime}, 5^{\prime}}=7.3 \mathrm{~Hz}\right), 5.43\left(\mathrm{q}, 1 \mathrm{H}, \mathrm{H}-2^{\prime}, J_{2^{\prime}, 1^{\prime}}=5.5 \mathrm{~Hz}\right), 4.66\left(\mathrm{~m}, 2 \mathrm{H}, \mathrm{H}-\mathrm{OCH} H_{2} \mathrm{O}\right), 4.19\left(\mathrm{dt}, 1 \mathrm{H}, \mathrm{H}-5, J_{5,6}=7.4, J_{5,4}=5.0 \mathrm{~Hz}\right)$, 3.99 (m, 2H, H-3, H-6), 3.35 (s, 3H, H-OCH $\mathrm{OCH}_{3}$ ), 2.75 (dq, 1H, H-2, $J_{2,3}=14.2, J_{2,8}=7.4 \mathrm{~Hz}$ ), 2.54 (ddq, 1H, H$\left.5^{\prime}, J_{g e m}=16.5, J_{5^{\prime}, 4^{\prime}}=6.9, J_{5^{\prime}, 6^{\prime}}=2.3 \mathrm{~Hz}\right), 2.41\left(\mathrm{ddq}, 1 \mathrm{H}, \mathrm{H}-5^{\prime}, J_{g e m}=16.5, J_{5^{\prime}, 4^{\prime}}=7.8, J_{5^{\prime}, 6^{\prime}}=2.8 \mathrm{~Hz}\right), 2.08(\mathrm{dt}, 1 \mathrm{H}, \mathrm{H}-$ 4, $\left.J_{g e m}=14.6, J_{4,3}=6.9 \mathrm{~Hz}\right), 1.95\left(\mathrm{dt}, 1 \mathrm{H}, \mathrm{H}-4, J_{g e m}=14.6, J_{4,5}=5.0 \mathrm{~Hz}\right), 1.74\left(\mathrm{t}, 3 \mathrm{H}, \mathrm{H}-6^{\prime}, J_{6^{\prime}, 5^{\prime}}=2.8 \mathrm{~Hz}\right), 1.67(\mathrm{~s}$, 3H, H-3'), 1.47 (s, 3H, H-OC( $\left.\left(\mathrm{CH}_{3}\right)_{2} \mathrm{O}\right), 1.40$ (s, 3H, H-9), 1.19 (d, 3H, H-8, $J_{8,2}=7.4 \mathrm{~Hz}$ ); ESIMS-LR m/z 465 $\left[(\mathrm{M}+\mathrm{MeOH}+\mathrm{Na})^{+}\right]$. 


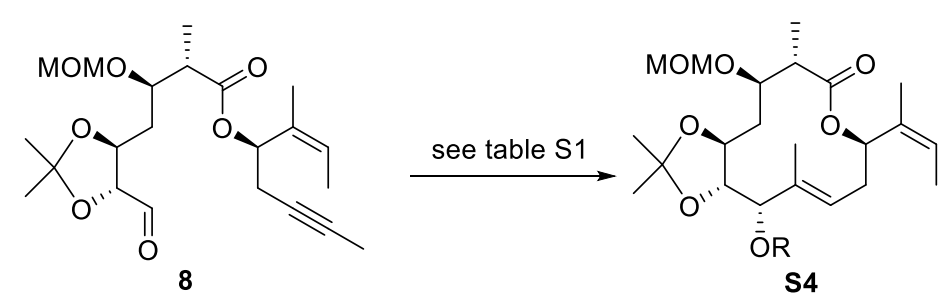

\begin{tabular}{|c|c|c|c|c|c|c|c|c|}
\hline entry & $\begin{array}{l}\mathrm{Ni(cod)})_{2} \\
(\mathrm{~mol} \%)\end{array}$ & $\begin{array}{l}\text { ligand } \\
(\mathrm{mol} \%)\end{array}$ & $\begin{array}{l}\text { reductant } \\
\text { (eq.) }\end{array}$ & temp. & $\begin{array}{l}\text { additive } \\
\text { (eq.) }\end{array}$ & $\begin{array}{l}\text { solvent } \\
(\mathrm{mM})\end{array}$ & $\mathrm{R}$ & yield $(\%)^{*}$ \\
\hline 1 & 20 & $\mathrm{Me}_{3} \mathrm{P}(40)$ & $\mathrm{Et}_{3} \mathrm{~B}(3)$ & $\mathrm{rt}$ & - & toluene $(5)$ & $\mathrm{H}$ & - \\
\hline 2 & 50 & $\operatorname{IPr}(50),{ }^{t} \mathrm{BuOK}(50)$ & $\mathrm{Et}_{3} \mathrm{SiH}(2)$ & $\mathrm{rt}$ & - & THF (5) & TES & - \\
\hline 3 & 50 & SIMes $\cdot \mathrm{HCl}(50),{ }^{t} \mathrm{BuOK}(50)$ & $\mathrm{Et}_{3} \mathrm{SiH}(2)$ & $\mathrm{rt}$ & - & THF (5) & TES & - \\
\hline 4 & 50 & IMes $\cdot \mathrm{HCl}(50),{ }^{t} \mathrm{BuOK}(50)$ & $\mathrm{Et}_{3} \mathrm{SiH}(2)$ & rt & - & THF (5) & TES & 8 \\
\hline 5 & 50 & IMes $\cdot \mathrm{HCl}(50),{ }^{t} \mathrm{BuOK}(50)$ & $\mathrm{Et}_{3} \mathrm{SiH}(2)$ & $40^{\circ} \mathrm{C}$ & - & THF (5) & TES & 23 \\
\hline 6 & 50 & IMes $\cdot \mathrm{HCl}(50),{ }^{t} \mathrm{BuOK}(50)$ & $\mathrm{Et}_{3} \mathrm{SiH}(2)$ & $50^{\circ} \mathrm{C}$ & - & THF (5) & TES & 28 \\
\hline 7 & 50 & IMes $\cdot \mathrm{HCl}(50),{ }^{t} \mathrm{BuOK}(50)$ & ${ }^{i} \mathrm{Pr}_{3} \mathrm{SiH}(2)$ & $50^{\circ} \mathrm{C}$ & - & THF (5) & TIPS & - \\
\hline 8 & 50 & IMes $\cdot \mathrm{HCl}(50),{ }^{t} \mathrm{BuOK}(50)$ & $\mathrm{Ph}_{3} \mathrm{SiH}(2)$ & $50^{\circ} \mathrm{C}$ & - & THF (5) & TPS & - \\
\hline 9 & 50 & IMes $\cdot \mathrm{HCl}(50),{ }^{t} \mathrm{BuOK}(50)$ & $\mathrm{Et}_{3} \mathrm{SiH}(2)$ & $50^{\circ} \mathrm{C}$ & - & toluene (5) & TES & 14 \\
\hline 10 & 50 & IMes $\cdot \mathrm{HCl}(50),{ }^{t} \mathrm{BuOK}(50)$ & $\mathrm{Et}_{3} \mathrm{SiH}(2)$ & $50^{\circ} \mathrm{C}$ & - & THF (2) & TES & 24 \\
\hline 11 & 50 & IMes $\cdot \mathrm{HCl}(50),{ }^{t} \mathrm{BuOK}(50)$ & $\mathrm{Et}_{3} \mathrm{SiH}(2)$ & $50^{\circ} \mathrm{C}$ & $\mathrm{Al}\left(\mathrm{O}^{\prime} \mathrm{Pr}\right)_{3}(2)$ & THF (5) & TES & 5 \\
\hline 12 & 50 & IMes $\cdot \mathrm{HCl}(50),{ }^{t} \mathrm{BuOK}(50)$ & $\mathrm{Et}_{3} \mathrm{SiH}(2)$ & $50^{\circ} \mathrm{C}$ & $\mathrm{Ti}\left(\mathrm{O}^{i} \mathrm{Pr}\right)_{4}(2)$ & THF (5) & TES & 3 \\
\hline 13 & 50 & IMes $\cdot \mathrm{HCl}(50),{ }^{t} \mathrm{BuOK}(50)$ & $\mathrm{Et}_{3} \mathrm{SiH}(2)$ & $50^{\circ} \mathrm{C}$ & $\mathrm{H}_{2} \mathrm{O}(2)$ & THF (5) & TES & 11 \\
\hline 14 & 50 & IMes $\cdot \mathrm{HCl}(50),{ }^{t} \mathrm{BuOK}(50)$ & $\mathrm{Et}_{3} \mathrm{SiH}(2)$ & $50^{\circ} \mathrm{C}$ & MS4A & THF (5) & TES & 19 \\
\hline 15 & 30 & IMes $\cdot \mathrm{HCl}(30),{ }^{t} \mathrm{BuOK}(30)$ & $\mathrm{Et}_{3} \mathrm{SiH}(2)$ & $50^{\circ} \mathrm{C}$ & - & THF (5) & TES & 16 \\
\hline 16 & 100 & IMes $\cdot \mathrm{HCl}(100),{ }^{t} \mathrm{BuOK}(100)$ & $\mathrm{Et}_{3} \mathrm{SiH}(2)$ & $50^{\circ} \mathrm{C}$ & - & THF (5) & TES & 5 \\
\hline $17^{* *}$ & 50 & IMes $\cdot \mathrm{HCl}(50),{ }^{t} \mathrm{BuOK}(50)$ & $\mathrm{Et}_{3} \mathrm{SiH}(2)$ & $50^{\circ} \mathrm{C}$ & - & THF (5) & TES & 33 \\
\hline
\end{tabular}

* Determined by ${ }^{1} \mathrm{H}$ NMR analysis of the crude mixture using 1,1,2,2-tetrachloroethane as the internal standard.

${ }^{* *} \mathrm{Ni}(\mathrm{cod})_{2}$, ligand and ${ }^{\mathrm{t}} \mathrm{BuOK}$ were added to a solution of $\mathbf{8}$ and reductant in solvent dropwise over $1 \mathrm{~h}$ in two portions.

$(3 \mathrm{a} S, 5 R, 6 S, 9 R, 13 S, 13 \mathrm{a} S, E)-9-[(Z)-B u t-2-e n-2-y l]-5-m e t h o x y m e t h o x y-2,2,6,12-t e t r a m e t h y l-13-$ triethylsilyloxy-3a,4,5,6,9,10,13,13a-octahydro-7H-[1,3]dioxolo[4,5-f][1] oxacyclododecin-7-one (S4)

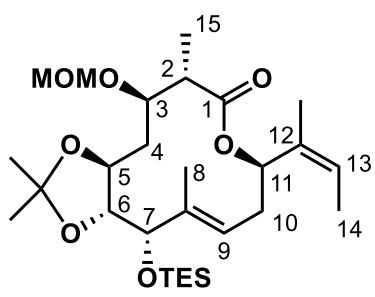

A solution of $\mathrm{Ni}(\operatorname{cod})_{2}(33 \mathrm{mg}, 0.12 \mathrm{mmol}), \mathrm{IMes} \cdot \mathrm{HCl}(41 \mathrm{mg}, 0.12 \mathrm{mmol})$ and ${ }^{t} \mathrm{BuOK}(13 \mathrm{mg}, 0.12 \mathrm{mmol})$ in THF $(5 \mathrm{~mL})$ was stirred at room temperature for $10 \mathrm{~min}$. A solution of the crude $8(100 \mathrm{mg})$ in THF $(43 \mathrm{~mL})$ was warmed to $50{ }^{\circ} \mathrm{C}$ and $\mathrm{Et}_{3} \mathrm{SiH}(77$ $\mu \mathrm{L}, 0.48 \mathrm{mmol})$ and the Ni solution $(2.5 \mathrm{~mL}, 0.06 \mathrm{mmol})$ were added to the mixture dropwise over $1 \mathrm{~h}$, and the whole mixture was stirred for $2 \mathrm{~h}$. The additional Ni solution 
$(2.5 \mathrm{~mL}, 0.06 \mathrm{mmol})$ was added to the mixture dropwise over $1 \mathrm{~h}$, and the mixture was stirred for $1 \mathrm{~h}$. The mixture was diluted with hexane, filtered through a short silica gel column and concentrated in vacuo. The residue was semipurified by flash silica gel column chromatography $(\phi 2 \times 10 \mathrm{~cm}, 10 \%$ EtOAc/hexane) to afford a mixture of $\mathbf{S} 4$ and ligand (50 mg, S4:ligand $=1: 0.4)$ as a colorless oil. The crude $\mathbf{S} 4$ was used for the next reaction without further purification. ${ }^{1} \mathrm{H}$ NMR $\left(\mathrm{CDCl}_{3}, 400 \mathrm{MHz}\right) \delta 6.06\left(\mathrm{dd}, 1 \mathrm{H}, \mathrm{H}-11, J_{11,10}=11.4, J_{11,10}=1.8 \mathrm{~Hz}\right), 5.67\left(\mathrm{~d}, 1 \mathrm{H}, \mathrm{H}-9, J_{9,10}\right.$ $=11.4 \mathrm{~Hz}), 5.36\left(\mathrm{q}, 1 \mathrm{H}, \mathrm{H}-13, J_{13,14}=6.9 \mathrm{~Hz}\right), 4.94\left(\mathrm{~d}, 1 \mathrm{H}, \mathrm{H}-\mathrm{OCH}_{2} \mathrm{O}, J_{\text {gem }}=6.4 \mathrm{~Hz}\right), 4.57\left(\mathrm{~d}, 1 \mathrm{H}, \mathrm{H}-\mathrm{OCH} \mathrm{H}_{2} \mathrm{O}, J_{g e m}\right.$ $=6.4 \mathrm{~Hz}), 4.27(\mathrm{br} \mathrm{s}, 1 \mathrm{H}, \mathrm{H}-7), 3.90\left(\mathrm{dd}, 1 \mathrm{H}, \mathrm{H}-5, J_{5,4}=10.1, J_{5,6}=8.2 \mathrm{~Hz}\right), 3.68\left(\mathrm{dt}, 1 \mathrm{H}, \mathrm{H}-3, J_{3,2}=10.3, J_{3,4}=4.1\right.$ $\mathrm{Hz}$ ), 3.61 (dd, $\left.1 \mathrm{H}, \mathrm{H}-6, J_{6,5}=8.2, J_{6,7}=2.3 \mathrm{~Hz}\right), 3.41$ (s, 3H, H-OCH $\mathrm{OCH}_{3}$ ), 2.73 (ddd, $1 \mathrm{H}, \mathrm{H}-10, J_{\text {gem }}=15.1, J_{10,11}$ $\left.=J_{10,9}=11.4 \mathrm{~Hz}\right), 1.97(\mathrm{~m}, 1 \mathrm{H}, \mathrm{H}-4), 1.95(\mathrm{~s}, 3 \mathrm{H}, \mathrm{H}-8), 1.85\left(\mathrm{dd}, 1 \mathrm{H}, \mathrm{H}-10, J_{g e m}=15.1, J_{10,11}=1.8 \mathrm{~Hz}\right), 1.74(\mathrm{~m}, 3 \mathrm{H}$, $\mathrm{H}-12), 1.68$ (d, 3H, H-14, $\left.J_{14,13}=6.9 \mathrm{~Hz}\right), 1.59$ (m, 1H, H-4), 1.37 (s, 6H, H-OC(CH $\left.)_{2} \mathrm{O}\right), 1.21\left(\mathrm{~d}, 3 \mathrm{H}, \mathrm{H}-15, J_{15,2}=\right.$ $6.9 \mathrm{~Hz}$ ), 0.95 (t, 9H, H-SiCH $\mathrm{CH}_{3}, J=8.2 \mathrm{~Hz}$ ), 0.60 (q, 6H, H-SiCH${ }_{2} \mathrm{CH}_{3}, J=8.2 \mathrm{~Hz}$ ); ESIMS-LR m/z 549 [(M+Na) $]$.

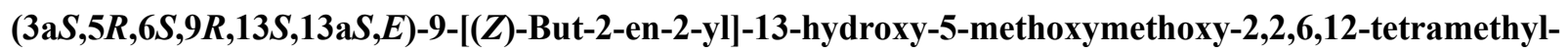

\section{3a,4,5,6,9,10,13,13a-octahydro-7H-[1,3]dioxolo[4,5-f][1] oxacyclododecin-7-one (34)}

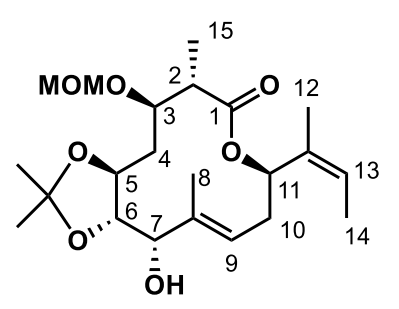

A solution of $\mathbf{S} 4$ in THF $(1 \mathrm{~mL})$ was treated with TBAF $(40 \mu \mathrm{L}, 40 \mu \mathrm{mol}, 1 \mathrm{M}$ in THF) at room temperature for $5 \mathrm{~h}$. The mixture was partitioned between EtOAc and $\mathrm{H}_{2} \mathrm{O}$. The organic phase was washed with $1 \mathrm{M} a q$. $\mathrm{HCl}$, sat. aq. $\mathrm{NaHCO}_{3}, \mathrm{H}_{2} \mathrm{O}$ and brine, dried $\left(\mathrm{Na}_{2} \mathrm{SO}_{4}\right)$, filtered and concentrated in vacuo. The residue was purified by hi-flash silica gel column chromatography $(\phi 1 \times 8 \mathrm{~cm}, 50 \%$ EtOAc/hexane) to afford 34 (30 $\mathrm{mg}, 73$ $\mu \mathrm{mol}, 30 \%$ over 3 steps) as a colorless oil. ${ }^{1} \mathrm{H} \mathrm{NMR}\left(\mathrm{CDCl}_{3}, 400 \mathrm{MHz}\right) \delta 6.08\left(\mathrm{dd}, 1 \mathrm{H}, \mathrm{H}-11, J_{11,10}=11.9, J_{11,10}=\right.$ $2.3 \mathrm{~Hz}$ ), 5.79 (d, 1H, H-9, $\left.J_{9,10}=11.9 \mathrm{~Hz}\right), 5.35$ (q, 1H, H-13, $\left.J_{13,14}=6.9 \mathrm{~Hz}\right), 4.89$ (d, 1H, H-OCH $\left.\mathrm{H}_{2} \mathrm{O} J_{g e m}=6.4 \mathrm{~Hz}\right)$, $4.57\left(\mathrm{~d}, 1 \mathrm{H}, \mathrm{H}-\mathrm{OCH} \mathrm{H}_{2} \mathrm{O}, J_{\text {gem }}=6.4 \mathrm{~Hz}\right), 4.31(\mathrm{br} \mathrm{s}, 1 \mathrm{H}, \mathrm{H}-7), 3.78(\mathrm{~m}, 1 \mathrm{H}, \mathrm{H}-6), 3.75(\mathrm{~m}, 1 \mathrm{H}, \mathrm{H}-5), 3.70$ (m, 1H, H3), 3.41 (s, 3H, $\mathrm{H}-\mathrm{OCH}_{2} \mathrm{OCH}_{3}$ ), 2.76 (ddd, $1 \mathrm{H}, \mathrm{H}-10, J_{\text {gem }}=14.9, J_{10,9}=J_{10,11}=11.9 \mathrm{~Hz}$ ), 2.63 (br s, 1H, H-OH), 2.44 $\left(\mathrm{dq}, 1 \mathrm{H}, \mathrm{H}-2, J_{2,3}=10.5, J_{2,15}=6.9 \mathrm{~Hz}\right), 2.00\left(\mathrm{dd}, 1 \mathrm{H}, \mathrm{H}-10, J_{\text {gem }}=14.9, J_{10,11}=2.3 \mathrm{~Hz}\right), 1.81\left(\mathrm{dd}, 1 \mathrm{H}, \mathrm{H}-4, J_{4,5}=\right.$ 15.1, $\left.J_{4,3}=6.4 \mathrm{~Hz}\right), 1.74(\mathrm{~m}, 4 \mathrm{H}, \mathrm{H}-4, \mathrm{H}-12), 1.68$ (d, 3H, H-14, $\left.J_{14,13}=6.9 \mathrm{~Hz}\right), 1.63$ (s, 3H, H-8), 1.43 (s, 3H, H$\left.\mathrm{OC}\left(\mathrm{CH}_{3}\right)_{2} \mathrm{O}\right), 1.41\left(\mathrm{~s}, 3 \mathrm{H}, \mathrm{H}-\mathrm{OC}\left(\mathrm{CH}_{3}\right)_{2} \mathrm{O}\right), 1.21\left(\mathrm{~d}, 3 \mathrm{H}, \mathrm{H}-15, J_{15,2}=6.9 \mathrm{~Hz}\right) ;{ }^{13} \mathrm{C} \mathrm{NMR}\left(\mathrm{CDCl}_{3}, 100 \mathrm{MHz}\right) \delta 173.9$, 134.0, 132.6, 123.1, 122.0, 109.1, 95.0, 94.7, 80.5, 74.5, 71.2, 56.3, 47.7, 42.1, 32.4, 32.3, 27.5, 27.1, 18.9, 15.9, 15.1, 13.3; ESIMS-LR m/z $435\left[(\mathrm{M}+\mathrm{Na})^{+}\right]$; HRMS (ESI) $\mathrm{m} / \mathrm{z}$ : $\left[(\mathrm{M}+\mathrm{Na})^{+}\right]$Calcd for $\mathrm{C}_{22} \mathrm{H}_{36} \mathrm{O}_{7} \mathrm{Na}$ 435.2359; Found 435.2375; $[\alpha]^{20}{ }_{\mathrm{D}}-149.6\left(c 1.02, \mathrm{CHCl}_{3}\right)$. Stereochemistry at the $\mathrm{C} 7$ position of $\mathbf{3 4}$ was determined by NOE correlation and conformational search using MacroModel (Figure S1). 
$(3 \mathrm{a} S, 5 R, 6 S, 9 R, 13 R, 13 \mathrm{a} R, E)-9-[(Z)-B u t-2-e n-2-y l]-5-m e t h o x y m e t h o x y-2,2,6,12-t e t r a m e t h y l-7-0 x 0-$

3a,4,6,7,9,10,13,13a-octahydro-5H-[1,3] dioxolo[4,5-f][1] oxacyclododecin-13-yl 4-nitrobenzoate (S5)

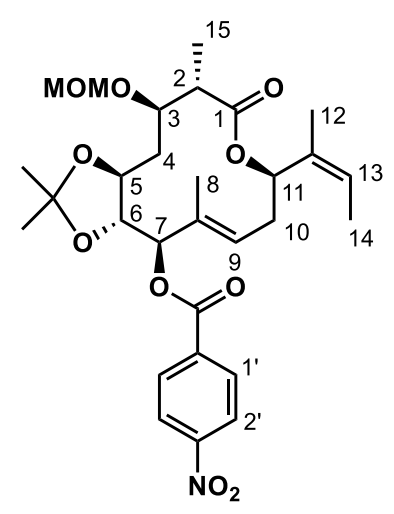

A solution of $34(10 \mathrm{mg}, 24 \mu \mathrm{mol})$ and $p$-nitrobenzoic acid $(6 \mathrm{mg}, 36 \mu \mathrm{mol})$ in toluene $(0.5 \mathrm{~mL})$ was treated with $\mathrm{PhOPPh}_{2}(10 \mathrm{mg}, 36 \mu \mathrm{mol})$ and bis(2-methoxyethyl) Azodicarboxylate (DMEAD) $(8.4 \mathrm{mg}, 36 \mu \mathrm{mol})$ at $0{ }^{\circ} \mathrm{C}$. The mixture was warmed to room temperature and stirred for $1 \mathrm{~h}$. The mixture was concentrated in vacuo. The residue was purified by flash silica gel column chromatography $(\phi 1 \times 8 \mathrm{~cm}, 13 \%$ EtOAc/hexane) to afford $\mathbf{S 5}(9.0 \mathrm{mg}, 16 \mu \mathrm{mol}, 67 \%)$ as a white solid. ${ }^{1} \mathrm{H} \mathrm{NMR}\left(\mathrm{CDCl}_{3}\right.$, $400 \mathrm{MHz}) \delta 8.28\left(\mathrm{~d}, 2 \mathrm{H}, \mathrm{H}-2^{\prime}, J_{2^{\prime}, 1^{\prime}}=8.7 \mathrm{~Hz}\right), 8.20\left(\mathrm{~d}, 2 \mathrm{H}, \mathrm{H}-1^{\prime}, J_{1^{\prime}, 2^{\prime}}=8.7 \mathrm{~Hz}\right), 6.07(\mathrm{~d}$, $\left.1 \mathrm{H}, \mathrm{H}-11, J_{11,10}=10.5 \mathrm{~Hz}\right), 5.78\left(\mathrm{~d}, 1 \mathrm{H}, \mathrm{H}-9, J_{9,10}=10.1 \mathrm{~Hz}\right), 5.41\left(\mathrm{~d}, 1 \mathrm{H}, \mathrm{H}-7, J_{7,6}=\right.$ $9.2 \mathrm{~Hz}), 5.36\left(\mathrm{q}, 1 \mathrm{H}, \mathrm{H}-13, J_{13,14}=8.2 \mathrm{~Hz}\right), 4.90\left(\mathrm{~d}, 1 \mathrm{H}, \mathrm{H}-\mathrm{OCH}_{2} \mathrm{O}, J_{\text {gem }}=6.9 \mathrm{~Hz}\right), 4.58\left(\mathrm{~d}, 1 \mathrm{H}, \mathrm{H}-\mathrm{OC} H_{2} \mathrm{O}, J_{g e m}=\right.$ $6.9 \mathrm{~Hz}), 3.89\left(\mathrm{t}, 1 \mathrm{H}, \mathrm{H}-6, J_{6,7}=J_{6,5}=9.2 \mathrm{~Hz}\right), 3.78\left(\mathrm{dd}, 1 \mathrm{H}, \mathrm{H}-3, J_{3,2}=10.5, J_{3,4}=6.0 \mathrm{~Hz}\right), 3.68\left(\mathrm{dd}, 1 \mathrm{H}, \mathrm{H}-5, J_{5,4}=\right.$ $J_{5,6}=9.2 \mathrm{~Hz}$ ), $3.43\left(\mathrm{~s}, 3 \mathrm{H}, \mathrm{H}-\mathrm{OCH}_{2} \mathrm{OCH}_{3}\right), 2.73\left(\mathrm{ddd}, 1 \mathrm{H}, \mathrm{H}-10, J_{g e m}=14.7, J_{10,11}=10.5, J_{10,9}=10.1 \mathrm{~Hz}\right), 2.48(\mathrm{dq}$, $\left.1 \mathrm{H}, \mathrm{H}-2, J_{2,3}=10.5, J_{2,15}=6.9 \mathrm{~Hz}\right), 2.04\left(\mathrm{~d}, 1 \mathrm{H}, \mathrm{H}-10, J_{g e m}=14.7 \mathrm{~Hz}\right), 1.86\left(\mathrm{dd}, 1 \mathrm{H}, \mathrm{H}-4, J_{\text {gem }}=14.6, J_{4,3}=6.0 \mathrm{~Hz}\right)$, 1.77 (m, 10H, H-4, H-8, H-12, H-14), 1.39 (s, 6H, H-OC $\left.\left(\mathrm{CH}_{3}\right)_{2} \mathrm{O}\right), 1.24$ (d, 3H, H-15, $\left.J_{15,2}=6.9 \mathrm{~Hz}\right) ;{ }^{13} \mathrm{C}$ NMR $\left(\mathrm{CDCl}_{3}, 100 \mathrm{MHz}\right) \delta 174.1,163.7,150.6,135.9,133.6,131.3,131.0,130.0,123.6,109.8,94.8,84.2,79.5,75.4,70.8$, 56.3, 47.7, 41.0, 32.6, 27.4, 26.7, 18.6, 16.0, 13.2, 12.2, 0.08; ESIMS-LR $m / z 584$ [(M+Na) $\left.{ }^{+}\right]$; HRMS (ESI) m/z: $\left[(\mathrm{M}+\mathrm{Na})^{+}\right]$Calcd for $\mathrm{C}_{29} \mathrm{H}_{39} \mathrm{NO}_{10} \mathrm{Na} 584.2472$; Found 584.2471; $[\alpha]^{20}{ }_{\mathrm{D}}-54.0\left(c\right.$ 0.86, $\left.\mathrm{CHCl}_{3}\right)$.

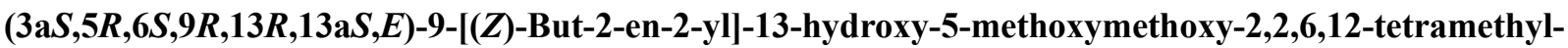

\section{3a,4,5,6,9,10,13,13a-octahydro-7H-[1,3]dioxolo[4,5-f][1] oxacyclododecin-7-one (6)}

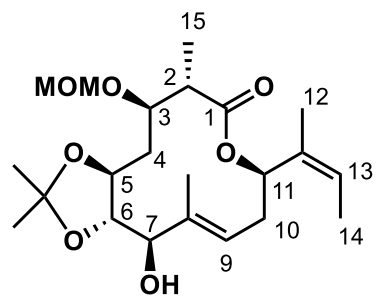

A solution of $\mathbf{S 5}(9.0 \mathrm{mg}, 16 \mu \mathrm{mol})$ in $\mathrm{MeOH}(0.5 \mathrm{~mL})$ was treated with $\mathrm{LiOH}(0.7 \mathrm{mg}$, $16 \mu \mathrm{mol})$ at $0{ }^{\circ} \mathrm{C}$ for $30 \mathrm{~min}$. The reaction was quenched with $1 \mathrm{M} \mathrm{aq}$. $\mathrm{HCl}$. The mixture was partitioned between EtOAc and $\mathrm{H}_{2} \mathrm{O}$. The organic phase was washed with $1 \mathrm{M} a q$. $\mathrm{HCl}$, sat. aq. $\mathrm{NaHCO}_{3}, \mathrm{H}_{2} \mathrm{O}$ and brine, dried $\left(\mathrm{Na}_{2} \mathrm{SO}_{4}\right)$, filtered and concentrated in vacuo. The residue was purified by flash silica gel column chromatography $(\phi 1 \times 8 \mathrm{~cm}, 20 \%$ EtOAc/hexane) to afford $6\left(6.6 \mathrm{mg}, 16 \mu \mathrm{mol}\right.$, quant.) as a colorless oil. ${ }^{1} \mathrm{H}$ NMR $\left(\mathrm{CDCl}_{3}, 400 \mathrm{MHz}\right) \delta 6.05(\mathrm{~d}, 1 \mathrm{H}$ $\left.\mathrm{H}-11, J_{11,10}=10.1 \mathrm{~Hz}\right), 5.53\left(\mathrm{~d}, 1 \mathrm{H}, \mathrm{H}-9, J_{9,10}=10.1 \mathrm{~Hz}\right), 5.38\left(\mathrm{q}, 1 \mathrm{H}, \mathrm{H}-13, J_{13,14}=8.2 \mathrm{~Hz}\right), 4.90\left(\mathrm{~d}, 1 \mathrm{H}, \mathrm{H}-\mathrm{OCH} \mathrm{H}_{2} \mathrm{O}\right.$, $\left.J_{\text {gem }}=6.4 \mathrm{~Hz}\right), 4.57\left(\mathrm{~d}, 1 \mathrm{H}, \mathrm{H}-\mathrm{OCH}_{2} \mathrm{O}, J_{\text {gem }}=6.4 \mathrm{~Hz}\right), 3.97\left(\mathrm{~d}, 1 \mathrm{H}, \mathrm{H}-7, J_{7,6}=8.7 \mathrm{~Hz}\right), 3.71\left(\mathrm{dd}, 1 \mathrm{H}, \mathrm{H}-3, J_{3,2}=10.6\right.$, $\left.J_{3,4}=6.0 \mathrm{~Hz}\right), 3.59\left(\mathrm{dd}, 1 \mathrm{H}, \mathrm{H}-6, J_{6,5}=J_{6,7}=8.7 \mathrm{~Hz}\right), 3.53\left(\mathrm{q}, 1 \mathrm{H}, \mathrm{H}-5, J_{5,4}=6.9 \mathrm{~Hz}\right), 3.43\left(\mathrm{~s}, 3 \mathrm{H}, \mathrm{H}-\mathrm{OCH}_{2} \mathrm{OCH}_{3}\right)$, $2.75\left(\mathrm{ddd}, 1 \mathrm{H}, \mathrm{H}-10, J_{g e m}=14.9, J_{10,9}=11.9, J_{10,11}=10.1 \mathrm{~Hz}\right), 2.47\left(\mathrm{dq}, 1 \mathrm{H}, \mathrm{H}-2, J_{2,3}=10.6, J_{2,15}=6.9 \mathrm{~Hz}\right), 2.33(\mathrm{br}$ s, 1H, H-OH), 2.00 (d, 1H, H-10, Jgem = 14.9 Hz), 1.68-1.79 (m, 11H, H-4, H-8, H-12, H-14), 1.42 (s, 3H, H$\left.\mathrm{OC}\left(\mathrm{CH}_{3}\right)_{2} \mathrm{O}\right), 1.40\left(\mathrm{~s}, 3 \mathrm{H}, \mathrm{H}-\mathrm{OC}\left(\mathrm{CH}_{3}\right)_{2} \mathrm{O}\right), 1.23\left(\mathrm{~d}, 3 \mathrm{H}, \mathrm{H}-15, J_{15,2}=6.9 \mathrm{~Hz}\right) ;{ }^{13} \mathrm{C} \mathrm{NMR}\left(\mathrm{CDCl}_{3}, 100 \mathrm{MHz}\right) \delta 174.2$, 
135.0, 133.8, 127.2, 123.4, 109.4, 94.8, 82.0, 81.8, 75.7, 71.0, 56.3, 47.8, 41.0, 32.5, 27.4, 27.3, 18.7, 16.0, 13.3, 11.5; ESIMS-LR m/z $435\left[(\mathrm{M}+\mathrm{Na})^{+}\right]$; HRMS (ESI) m/z: $\left[(\mathrm{M}+\mathrm{Na})^{+}\right]$Calcd for $\mathrm{C}_{22} \mathrm{H}_{36} \mathrm{O}_{7} \mathrm{Na}$ 435.2359; Found $435.2375 ;[\alpha]^{20}{ }_{D}-7.3\left(c 1.10, \mathrm{CHCl}_{3}\right)$. Stereochemistry at the $\mathrm{C} 7$ position of $\mathbf{6}$ was determined by NOE correlation and conformational search using MacroModel (Figure S1).

$(2 R, 3 R, 4 S, 5 S)$-2-Acetoxymethyl-5-\{\{(3aS,5R,6S,9R,13R,13a $R, E)-9-[(Z)$-but-2-en-2-yl]-5-methoxymethoxy2,2,6,12-tetramethyl-7-oxo-3a,4,6,7,9,10,13,13a-octahydro-5H-[1,3]dioxolo[4,5-f][1] ]oxacyclododecin-13yl\}oxy\}tetrahydrofuran-3,4-diyl diacetate (35)

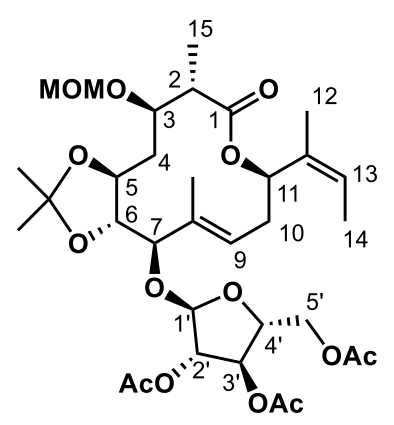

A solution of 6 (5 mg, $12 \mu \mathrm{mol}), 7(10 \mathrm{mg}, 24 \mu \mathrm{mol})$ and MS4A $(50 \mathrm{mg})$ in $\mathrm{CH}_{2} \mathrm{Cl}_{2}$ $(1 \mathrm{~mL})$ was treated with a solution of TMSOTf $(0.2 \mu \mathrm{L}, 1.2 \mu \mathrm{mol})$ in $\mathrm{CH}_{2} \mathrm{Cl}_{2}(0.2 \mathrm{~mL})$ at $-20{ }^{\circ} \mathrm{C}$. The mixture was warmed to $-7{ }^{\circ} \mathrm{C}$ and stirred for $30 \mathrm{~min}$. The reaction was quenched with sat. aq. $\mathrm{NaHCO}_{3}$. The mixture was partitioned between EtOAc and $\mathrm{H}_{2} \mathrm{O}$. The organic phase was washed with $1 \mathrm{M}$ aq. $\mathrm{HCl}$, sat. aq. $\mathrm{NaHCO}_{3}, \mathrm{H}_{2} \mathrm{O}$ and brine, dried $\left(\mathrm{Na}_{2} \mathrm{SO}_{4}\right)$, filtered and concentrated in vacuo. The residue was purified by flash silica gel column chromatography $(\phi 1 \times 8 \mathrm{~cm}, 20 \rightarrow 25 \%$ EtOAc/hexane) to afford $\mathbf{3 5}(8.0 \mathrm{mg}, 11.9$ $\mu$ mol mmol, $98 \%)$ as a colorless oil. ${ }^{1} \mathrm{H}$ NMR $\left(\mathrm{CDCl}_{3}, 400 \mathrm{MHz}\right) \delta 6.02\left(\mathrm{~d}, 1 \mathrm{H}, \mathrm{H}-11, J_{11,10}=9.6 \mathrm{~Hz}\right), 5.50(\mathrm{~d}, 1 \mathrm{H}$, $\left.\mathrm{H}-9, J_{9,10}=11.0 \mathrm{~Hz}\right), 5.35$ (q, 1H, H-13, $\left.J_{13,14}=6.9 \mathrm{~Hz}\right), 5.29$ (s, 1H, H-1'), 5.17 (s, 1H, H-2'), 4.90 (d, 1H, H-3', $J_{3^{\prime}, 4}$ $=3.6 \mathrm{~Hz}), 4.87\left(\mathrm{~d}, 1 \mathrm{H}, \mathrm{H}-\mathrm{OC} H_{2} \mathrm{O}, J_{g e m}=6.4 \mathrm{~Hz}\right), 4.55\left(\mathrm{~d}, 1 \mathrm{H}, \mathrm{H}-\mathrm{OCH} H_{2} \mathrm{O}, J_{g e m}=6.4 \mathrm{~Hz}\right), 4.35\left(\mathrm{dt}, 1 \mathrm{H}, \mathrm{H}-4^{\prime}, J_{4^{\prime}, 3^{\prime}}=\right.$ 3.6, $\left.J_{4^{\prime}, 5^{\prime}}=6.4 \mathrm{~Hz}\right), 4.17\left(\mathrm{~m}, 2 \mathrm{H}, \mathrm{H}-5^{\prime}\right), 3.99\left(\mathrm{~d}, 1 \mathrm{H}, \mathrm{H}-7, J_{7,6}=9.0 \mathrm{~Hz}\right), 3.71\left(\mathrm{dd}, 1 \mathrm{H}, \mathrm{H}-3, J_{3,2}=10.1, J_{3,4}=5.5 \mathrm{~Hz}\right)$, $3.67\left(\mathrm{dd}, 1 \mathrm{H}, \mathrm{H}-6, J_{6,7}=J_{6,5}=9.0 \mathrm{~Hz}\right), 3.49\left(\mathrm{dd}, 1 \mathrm{H}, \mathrm{H}-5, J_{5,6}=J_{5,4}=9.0 \mathrm{~Hz}\right), 3.40\left(\mathrm{~s}, 3 \mathrm{H}, \mathrm{H}-\mathrm{OCH}_{2} \mathrm{OCH}_{3}\right), 2.72$ $\left(\mathrm{ddd}, 1 \mathrm{H}, \mathrm{H}-10, J_{\text {gem }}=14.6, J_{10,9}=J_{10,11}=11.0\right), 2.45\left(\mathrm{dq}, 1 \mathrm{H}, \mathrm{H}-2, J_{2,3}=10.1, J_{2,15}=6.4 \mathrm{~Hz}\right), 2.11(\mathrm{~m}, 1 \mathrm{H}, \mathrm{H}-4)$, $2.10(\mathrm{~m}, 9 \mathrm{H}, \mathrm{H}-\mathrm{Ac}), 1.96$ (d, 1H, H-10, $\left.J_{\text {gem }}=14.6 \mathrm{~Hz}\right), 1.78\left(\mathrm{dd}, 1 \mathrm{H}, \mathrm{H}-4, J_{\text {gem }}=15.6, J_{4,3}=5.5 \mathrm{~Hz}\right), 1.74(\mathrm{~s}, 3 \mathrm{H}$, $\mathrm{H}-8), 1.67$ (d, 3H, H-14, $\left.J_{14,13}=6.9 \mathrm{~Hz}\right), 1.63(\mathrm{~s}, 3 \mathrm{H}, \mathrm{H}-12), 1.38\left(\mathrm{~s}, 3 \mathrm{H}, \mathrm{H}-\mathrm{OC}\left(\mathrm{CH}_{3}\right)_{2} \mathrm{O}\right), 1.36\left(\mathrm{~s}, 3 \mathrm{H}, \mathrm{H}-\mathrm{OC}(\mathrm{CH})_{2} \mathrm{O}\right)$, $1.23\left(\mathrm{~d}, 3 \mathrm{H}, \mathrm{H}-15, J_{15,2}=6.4 \mathrm{~Hz}\right) ;{ }^{13} \mathrm{C} \mathrm{NMR}\left(\mathrm{CDCl}_{3}, 100 \mathrm{MHz}\right) \delta 174.1,170.7,170.3,169.6,134.4,133.8,126.9$, 123.4, 109.2, 105.4, 94.8, 85.7, 81.5, 81.2, 80.9, 75.6, 70.8, 63.6, 56.3, 47.7, 40.1, 32.4, 30.1, 27.5, 27.1, 20.9, 18.6, 16.0, 13.3, 12.1; ESIMS-LR $m / z 693$ [(M+Na) $)^{+}$; HRMS (ESI) m/z: $\left[(\mathrm{M}+\mathrm{Na})^{+}\right]$Calcd for $\mathrm{C}_{33} \mathrm{H}_{50} \mathrm{O}_{14} \mathrm{Na} 693.3098$; Found 693.3110; $[\alpha]^{20}{ }_{\mathrm{D}}-40.5\left(c 0.60, \mathrm{CHCl}_{3}\right)$. 
Scheme S4. Synthesis of 7

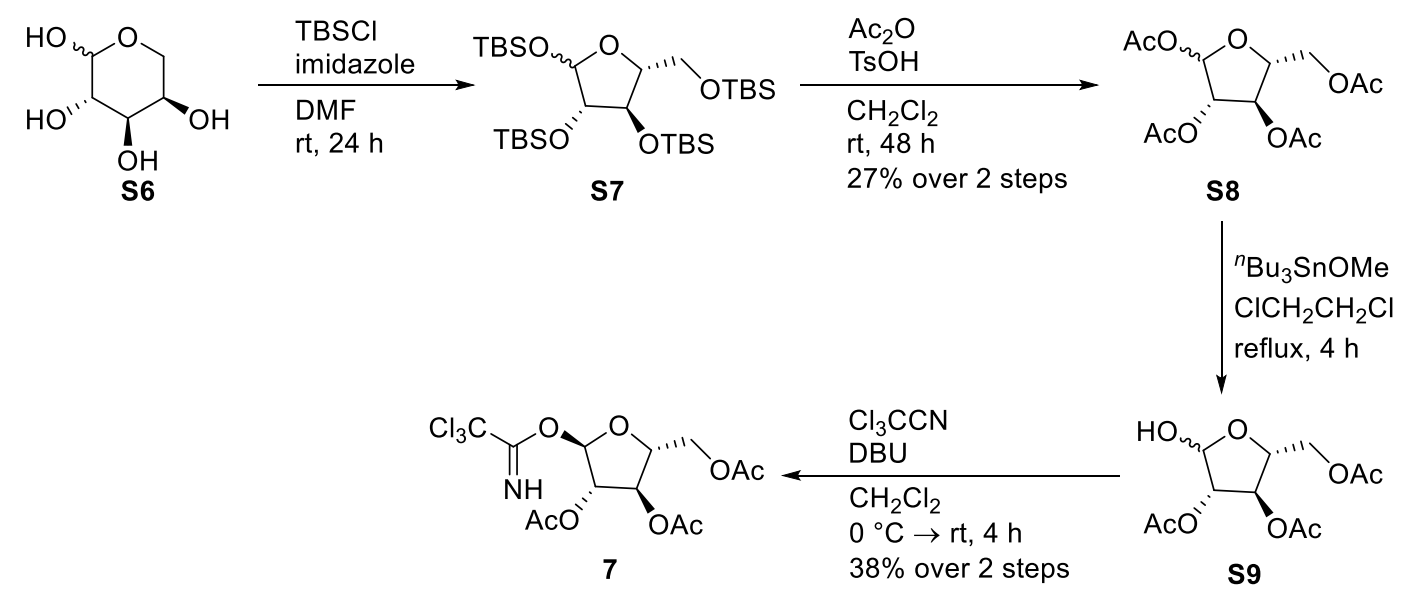

\section{1,2,3,5-Tetra-O-acetyl-D-arabinofuranose (S8)}

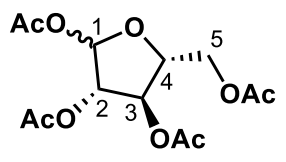

A solution of D-arabinose (S6) $(600 \mathrm{mg}, 4 \mathrm{mmol})$ in DMF $(40 \mathrm{~mL})$ was treated with imidazole $(680 \mathrm{mg}, 20 \mathrm{mmol})$ and $\mathrm{TBSCl}(1.8 \mathrm{~g}, 24 \mathrm{mmol})$ at room temperature for $24 \mathrm{~h}$. The mixture was filtered to afford a crude $\mathbf{S} 7$ as a white solid. A solution of the crude $\mathbf{S} 7$ (1.55 g) in $\mathrm{CH}_{2} \mathrm{Cl}_{2}$ (5 $\mathrm{mL})$ was treated with $\mathrm{TsOH}(3.8 \mathrm{~g}, 20 \mathrm{mmol})$ and $\mathrm{Ac}_{2} \mathrm{O}(3.78 \mathrm{~mL}, 40 \mathrm{mmol})$ at room temperature for $48 \mathrm{~h}$. The reaction was quenched with $\mathrm{Et}_{3} \mathrm{~N}$. The mixture was partitioned between $\mathrm{CH}_{2} \mathrm{Cl}_{2}$ and $\mathrm{H}_{2} \mathrm{O}$. The organic phase was washed with sat. aq. $\mathrm{NaHCO}_{3}, \mathrm{H}_{2} \mathrm{O}$ and brine, dried $\left(\mathrm{Na}_{2} \mathrm{SO}_{4}\right)$, filtered and concentrated in vacuo. The residue was purified by flash silica gel column chromatography $(\phi 4 \times 13 \mathrm{~cm}, 33 \%$ EtOAc/hexane) to afford an anomeric mixture S8 (341 mg, $1.07 \mathrm{mmol}, 27 \%$ over 2 steps, $\alpha / \beta=1: 5)$ as a colorless oil. Data for $\alpha$ anomer: ${ }^{1} \mathrm{H}$ NMR $\left(\mathrm{CDCl}_{3}, 400\right.$ MHz) $\delta 6.36$ (br s, 1H, H-1), 5.33 (m, 1H, H-1), 5.10 (m, 1H, H-3), 4.40 (m, 1H, H-4), 4.23 (m, 2H, H-5), 2.10 (m, $12 \mathrm{H}, \mathrm{Ac})$. Data for $\beta$ anomer: ${ }^{1} \mathrm{H} \mathrm{NMR}\left(\mathrm{CDCl}_{3}, 400 \mathrm{MHz}\right) \delta 6.18$ (s, 1H, H-1), 5.19 (s, 1H, H-2), 5.02 (m, 1H, H-3), $4.35(\mathrm{~m}, 1 \mathrm{H}, \mathrm{H}-4), 4.20(\mathrm{~m}, 2 \mathrm{H}, \mathrm{H}-5), 2.10(\mathrm{~m}, 12 \mathrm{H}, \mathrm{Ac})$; ESIMS-LR $m / z 341\left[(\mathrm{M}+\mathrm{Na})^{+}\right]$. This is a known compound. ${ }^{\mathrm{S} 7}$

\section{2,3,5-Tri-O-acetyl-a-L-arabinofuranosyl trichloroacetimidate (7)}

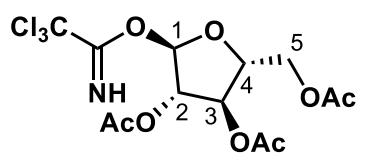

A solution of $\mathbf{S 8}(100 \mathrm{mg}, 0.31 \mathrm{mmol})$ in $\mathrm{ClCH}_{2} \mathrm{CH}_{2} \mathrm{Cl}(5 \mathrm{~mL})$ was treated with ${ }^{n} \mathrm{Bu}_{3} \mathrm{SnOMe}(182 \mu \mathrm{L}, 0.63 \mathrm{mmol})$ at room temperature. The mixture was warmed to reflux and stirred for $4 \mathrm{~h}$. The mixture was concentrated in vacuo. The residue was purified by silica gel column chromatography $(\phi 4 \times 10 \mathrm{~cm}, \mathrm{EtOAc} / \mathrm{hexane}=2: 3)$ to afford a crude $\mathbf{S 9}$. A solution of the crude S9 in $\mathrm{CH}_{2} \mathrm{Cl}_{2}(2 \mathrm{~mL})$ was treated with $\mathrm{Cl}_{3} \mathrm{CCN}(73 \mu \mathrm{L}, 0.73 \mathrm{mmol})$ and $\mathrm{DBU}(4 \mu \mathrm{L}, 0.029 \mathrm{mmol})$ at $0{ }^{\circ} \mathrm{C}$. The mixture was warmed to room temperature and stirred for $4 \mathrm{~h}$. The mixture was concentrated in vacuo. The residue was purified by flash silica gel column chromatography $\left(\phi 4 \times 10 \mathrm{~cm}, 25 \% \mathrm{EtOAc} /\right.$ hexane, $\left.0.1 \% \mathrm{Et}_{3} \mathrm{~N}\right)$ to afford $7(52 \mathrm{mg}, 0.12$ mmol, $38 \%$ over 2 steps) as a colorless oil. ${ }^{1} \mathrm{H}$ NMR $\left(\mathrm{CDCl}_{3}, 400 \mathrm{MHz}\right) \delta 8.62(\mathrm{~s}, 1 \mathrm{H}, \mathrm{NH}), 6.34(\mathrm{~s}, 1 \mathrm{H}, \mathrm{H}-1), 5.37$ 
(s, 1H, H-2), $5.04\left(\mathrm{~d}, 1 \mathrm{H}, \mathrm{H}-3, J_{3,4}=3.7 \mathrm{~Hz}\right), 4.44\left(\mathrm{ddd}, 1 \mathrm{H}, \mathrm{H}-4, J_{4,3}=3.7, J_{4,5}=4.1, J_{4,5}=5.5 \mathrm{~Hz}\right), 4.41(\mathrm{dd}, 1 \mathrm{H}$, $\left.\mathrm{H}-5, J_{\text {gem }}=11.9, J_{5,4}=4.1 \mathrm{~Hz}\right), 4.25\left(\mathrm{dd}, 1 \mathrm{H}, \mathrm{H}-5, J_{g e m}=11.9, J_{5,4}=5.5 \mathrm{~Hz}\right), 2.14(\mathrm{~s} 3 \mathrm{H}, \mathrm{Ac}), 2.06(\mathrm{~s}, 6 \mathrm{H}, \mathrm{Ac})$; ESIMS-LR $m / z 441\left[(\mathrm{M}+\mathrm{Na})^{+}\right]$. This is a known compound. ${ }^{\mathrm{S} 8}$

Scheme S5. Synthesis of $\mathbf{3 7}$

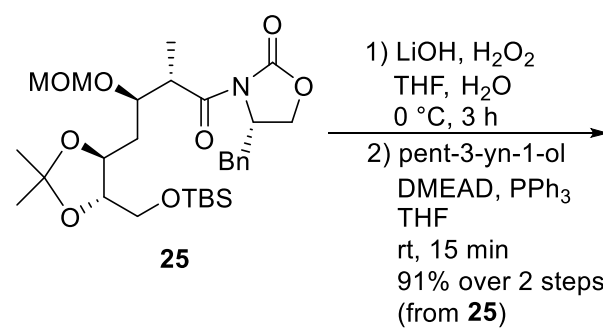

(from 25)

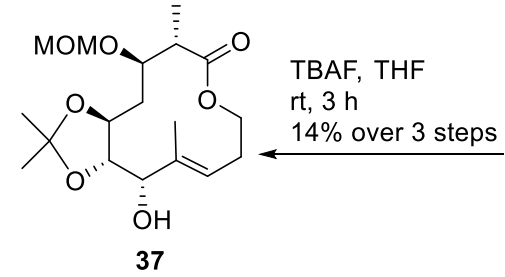

\begin{tabular}{|c|c|c|}
\hline entry & conditions & result \\
\hline 1 & $(\mathrm{COCl})_{2}, \mathrm{DMSO}, \mathrm{Et}_{3} \mathrm{~N}, \mathrm{CH}_{2} \mathrm{Cl}_{2},-78{ }^{\circ} \mathrm{C} \rightarrow \mathrm{rt}$ & complex mixture \\
\hline 2 & TEMPO, $\mathrm{Phl}(\mathrm{OAc})_{2}, \mathrm{MeCN}, \mathrm{pH} 7$ buffer, $0{ }^{\circ} \mathrm{C}$ & complex mixture \\
\hline 3 & TPAP, NMO, MS4A, $\mathrm{CH}_{2} \mathrm{Cl}_{2}, \mathrm{MeCN}$ & complex mixture \\
\hline 4 & $\mathrm{SO}_{3} \cdot \mathrm{Py}$., DMSO, $\mathrm{Et}_{3} \mathrm{~N}, \mathrm{CH}_{2} \mathrm{Cl}_{2}$ & complex mixture \\
\hline 5 & IBX, $\mathrm{NaHCO}_{3}, \mathrm{MeCN}, 80^{\circ} \mathrm{C}$ & complex mixture \\
\hline 6 & $\begin{array}{l}\text { Dess-Martin periodinane, } \mathrm{CH}_{2} \mathrm{Cl}_{2} \\
\quad \text { (aqueous workup) }\end{array}$ & complex mixture \\
\hline 7 & $\begin{array}{l}\text { Dess-Martin periodinane, } \mathrm{CH}_{2} \mathrm{Cl}_{2} \\
\text { (without aqueous workup) }\end{array}$ & quant. \\
\hline
\end{tabular}

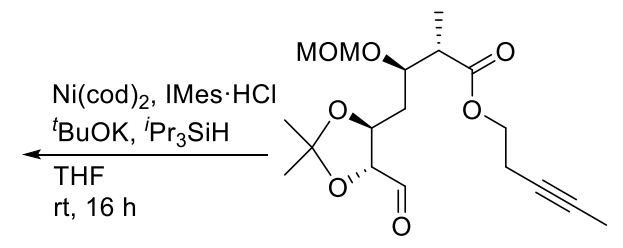

S11

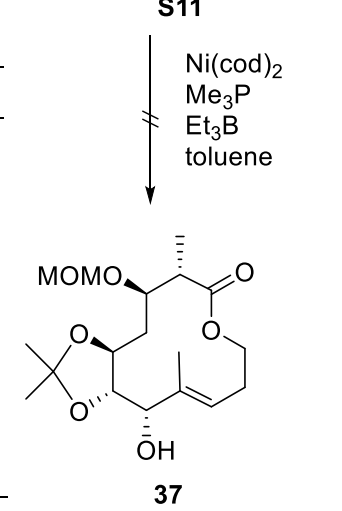

Pent-3-yn-1-yl (2S,3R)-4-[(4S,5S)-5-tert-butyldimethylsilyloxymethyl-2,2-dimethyl-1,3-dioxolan-4-yl]-3methoxymethoxy-2-methylbutanoate (S10)

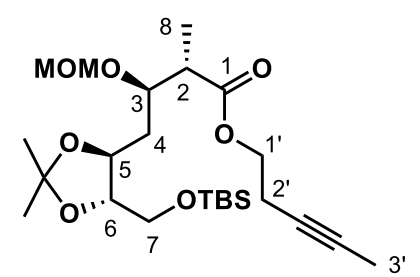

A solution of $25(1.80 \mathrm{~g}, 3.18 \mathrm{mmol})$ in THF $(24 \mathrm{~mL})$ and $\mathrm{H}_{2} \mathrm{O}(6 \mathrm{~mL})$ was treated with $\mathrm{H}_{2} \mathrm{O}_{2}\left(2.17 \mathrm{~mL}, 19.1 \mathrm{mmol}, 30 \%\right.$ in $\left.\mathrm{H}_{2} \mathrm{O}\right)$ and $\mathrm{LiOH}(401 \mathrm{mg}, 9.54 \mathrm{mmol})$ at $0{ }^{\circ} \mathrm{C}$ for 3 h. The reaction was quenched with sat. aq. $\mathrm{Na}_{2} \mathrm{~S}_{2} \mathrm{O}_{3}$. The mixture was partitioned between $\mathrm{Et}_{2} \mathrm{O}$ and sat. aq. $\mathrm{NaHCO}_{3}$. The organic phase was washed with brine, dried $\left(\mathrm{Na}_{2} \mathrm{SO}_{4}\right)$, filtered and concentrated in vacuo to give $\mathbf{9}$. A solution of 9 (1.12 g), pent3-yn-1-ol (424 $\mu \mathrm{L}, 4.64 \mathrm{mmol})$ and $\mathrm{PPh}_{3}(1.22 \mathrm{~g}, 4.64 \mathrm{mmol})$ was treated with DMEAD (1.09 g, $\left.4.64 \mathrm{mmol}\right)$ at room temperature for $15 \mathrm{~min}$. The mixture was concentrated in vacuo. The residue was purified by silica gel column 
chromatography ( $\phi 4 \times 15 \mathrm{~cm}, 10 \%$ EtOAc/hexane) to afford $\mathbf{S 1 0}(1.0 \mathrm{~g}, 2.12 \mathrm{mmol}, 91 \%$ over 2 steps $)$ as a colorless oil. ${ }^{1} \mathrm{H}$ NMR $\left(\mathrm{CDCl}_{3}, 400 \mathrm{MHz}\right) \delta 4.58$ (m, 2H, H-OCH $\left.\mathrm{O}\right), 4.08$ (m, 2H, H-1'), 4.05 (m, 1H, H-3), 3.88 (m, 1H, H5), 3.71 (m, 1H, H-6), 3.60 (m, 2H, H-7), 3.28 (s, 3H, H-OCH $\mathrm{OCH}_{3}$ ), 2.72 (m, 1H, H-2), 2.40 (m, 2H, H-2'), 1.87 $\left(\mathrm{dt}, 2 \mathrm{H}, \mathrm{H}-4, J_{4,3}=4.2, J_{4,5}=1.8 \mathrm{~Hz}\right), 1.71\left(\mathrm{~s}, 3 \mathrm{H}, \mathrm{H}-3^{\prime}\right), 1.32\left(\mathrm{~s}, 3 \mathrm{H}, \mathrm{H}-\mathrm{OC}\left(\mathrm{CH}_{3}\right)_{2} \mathrm{O}\right), 1.30\left(\mathrm{~s}, 3 \mathrm{H}, \mathrm{H}-\mathrm{OC}(\mathrm{CH})_{2} \mathrm{O}\right)$,

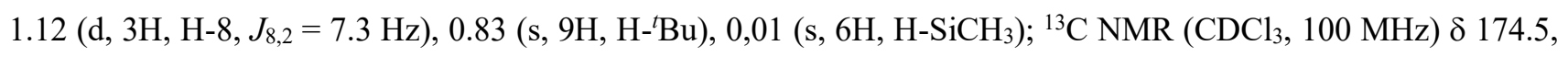
108.8, 96.6, 81.3, 75.8, 74.9, 63.5, 62.9, 55.9, 43.0, 36.0, 27.4, 27.0, 26.0, 19.3, 18.5, 11.6, 3.4, -5.3; ESIMS-LR $m / z$ $495\left[(\mathrm{M}+\mathrm{Na})^{+}\right]$; HRMS (ESI) m/z: $\left[(\mathrm{M}+\mathrm{Na})^{+}\right] \mathrm{Calcd}$ for $\mathrm{C}_{24} \mathrm{H}_{44} \mathrm{O}_{7} \mathrm{~N}_{1} \mathrm{NaSi} 495.2754$; Found 495.2751; $[\alpha]^{20}{ }_{\mathrm{D}}-3.6(c$ $\left.1.80, \mathrm{CHCl}_{3}\right)$.

\section{Pent-3-yn-1-yl (2S,3R)-4-[(4S,5S)-5-hydroxymethyl-2,2-dimethyl-1,3-dioxolan-4-yl]-3-methoxymethoxy-2-} methylbutanoate (36)

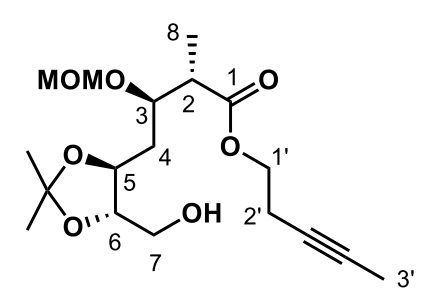

A solution of $\mathbf{S 1 0}(224 \mathrm{mg}, 0.47 \mathrm{mmol})$ in THF $(5 \mathrm{~mL})$ was treated with $3 \mathrm{HF}^{-\mathrm{Et}_{3} \mathrm{~N}}$ $(383 \mu \mathrm{L}, 2.35 \mathrm{mmol})$ at room temperature for $19 \mathrm{~h}$. The mixture was partitioned between EtOAc and $\mathrm{H}_{2} \mathrm{O}$. The organic phase was washed with $1 \mathrm{M}$ aq. $\mathrm{HCl}$, sat. aq. $\mathrm{NaHCO}_{3}, \mathrm{H}_{2} \mathrm{O}$ and brine, dried $\left(\mathrm{Na}_{2} \mathrm{SO}_{4}\right)$, filtered and concentrated in vacuo. The residue was purified by silica gel column chromatography $(\phi 3 \times 10 \mathrm{~cm}, 25 \%$ EtOAc/hexane) to afford $36(167 \mathrm{mg}, 0.47 \mathrm{mmol}, 99 \%)$ as a colorless oil. ${ }^{1} \mathrm{H} \mathrm{NMR}\left(\mathrm{CDCl}_{3}, 400 \mathrm{MHz}\right) \delta 4.68(\mathrm{~s}, 2 \mathrm{H}$, $\mathrm{H}-\mathrm{OCH} \mathrm{H}_{2} \mathrm{O}$ ), 4.15 (m, 2H, H-1'), 4.03 (m, 2H, H-3, H-5), 3.80 (m, 1H, H-7), 3.76 (dt, 1H, H-6, J6,5 = 8.2, J6,7 $=4.1$ $\mathrm{Hz}$ ), 3.65 (m, 1H, H-7), 3.37 (s, 3H, H-OCH $\mathrm{OCH}_{3}$ ), 2.78 (dq, 1H, H-2, $J_{2,3}=J_{2,8}=7.3 \mathrm{~Hz}$ ), 2.47 (m, 2H, H-2'), 2.10 (m, 1H, H-OH), $1.98\left(\mathrm{dt}, 1 \mathrm{H}, \mathrm{H}-4, J_{\text {gem }}=15.1, J_{4,3}=J_{4,5}=6.4 \mathrm{~Hz}\right), 1.85\left(\mathrm{dt}, 1 \mathrm{H}, \mathrm{H}-4, J_{\text {gem }}=15.1, J_{4,3}=J_{4,5}=5.5\right.$ $\mathrm{Hz}), 1.78\left(\mathrm{t}, 3 \mathrm{H}, \mathrm{H}-3^{\prime}, J_{3^{\prime}, 2^{\prime}}=2.3 \mathrm{~Hz}\right), 1.41$ (s, 3H, H-OC(CH$\left.)_{2} \mathrm{O}\right), 1.40\left(\mathrm{~s}, 3 \mathrm{H}, \mathrm{H}-\mathrm{OC}\left(\mathrm{CH}_{3}\right)_{2} \mathrm{O}\right), 1.20\left(\mathrm{~d}, 3 \mathrm{H}, \mathrm{H}-8, J_{8,2}\right.$ $=7.3 \mathrm{~Hz}) ;{ }^{13} \mathrm{C} \mathrm{NMR}\left(\mathrm{CDCl}_{3}, 100 \mathrm{MHz}\right) \delta 174.4,108.9,96.8,81.6,74.9,74.1,62.9,61.9,56.0,43.5,36.0,27.4,27.1$, 19.2, 12.2, 3.5; ESIMS-LR m/z $381\left[(\mathrm{M}+\mathrm{Na})^{+}\right]$; HRMS (ESI) m/z: $\left[(\mathrm{M}+\mathrm{Na})^{+}\right]$Calcd for $\mathrm{C}_{18} \mathrm{H}_{30} \mathrm{O}_{7} \mathrm{Na} 381.1902$; Found 381.1889; $[\alpha]^{20}{ }_{\mathrm{D}}+4.8\left(c 1.50, \mathrm{CHCl}_{3}\right)$.

\section{Pent-3-yn-1-yl (2S,3R)-4-[(4S,5R)-5-formyl-2,2-dimethyl-1,3-dioxolan-4-yl]-3-methoxymethoxy-2-} methylbutanoate (S11)

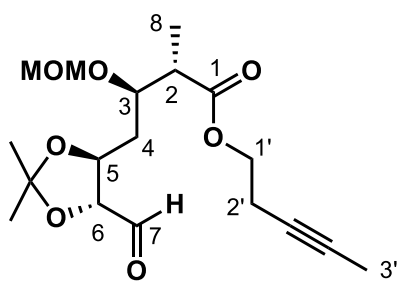

A solution of $\mathbf{3 6}(30 \mathrm{mg}, 84 \mu \mathrm{mol})$ in $\mathrm{CH}_{2} \mathrm{Cl}_{2}(0.8 \mathrm{~mL})$ was treated with Dess-Martin periodinane ( $54 \mathrm{mg}, 126 \mu \mathrm{mol})$ at room temperature for $30 \mathrm{~min}$. The mixture was diluted with hexane and filtered through a Cerite pad. The filtrate was concentrated in vacuo, and the residue was purified by silica gel column chromatography $(\phi 1 \times 8 \mathrm{~cm}, 40 \%$ EtOAc/hexane) to afford a crude S11 (30 mg) as a colorless oil. The crude aldehyde was used for the next reaction without further purification. ${ }^{1} \mathrm{H} \mathrm{NMR}\left(\mathrm{CDCl}_{3}, 400 \mathrm{MHz}\right) \delta 9.73(\mathrm{~s}, 1 \mathrm{H}, \mathrm{CHO}), 4.65\left(\mathrm{~d}, 1 \mathrm{H}, \mathrm{H}-\mathrm{OCH} \mathrm{H}_{2} \mathrm{O}, J_{\text {gem }}=7.0\right.$ 
$\mathrm{Hz}), 4.62\left(\mathrm{~d}, 1 \mathrm{H}, \mathrm{H}-\mathrm{OCH} H_{2} \mathrm{O}, J_{\text {gem }}=7.0 \mathrm{~Hz}\right), 4.16$ (m, 2H, H-1'), 4.15 (m, 1H, H-3), 4.11 (m, 1H, H-5), 4.00 (m, 1H, H-5), 3.36 (s, 3H, H-OCH $\mathrm{OCH}_{3}$ ), 2.77 (dq, 1H, H-2, $J_{2,3}=12.8, J_{2,8}=7.3 \mathrm{~Hz}$ ), 2.46 (m, 2H, H-2'), 2.06 (dt, 1H, H4, $\left.J_{\text {gem }}=15.0, J_{4,3}=6.6 \mathrm{~Hz}\right), 1.96\left(\mathrm{dt}, 1 \mathrm{H}, \mathrm{H}-4, J_{\text {gem }}=15.0,5.2 \mathrm{~Hz}\right), 1.77\left(\mathrm{t}, 3 \mathrm{H}, \mathrm{H}-3^{\prime}, J_{3^{\prime}, 2^{\prime}}=2.6 \mathrm{~Hz}\right), 1.47(\mathrm{~s}, 3 \mathrm{H}, \mathrm{H}-$ $\left.\mathrm{OC}\left(\mathrm{CH}_{3}\right)_{2} \mathrm{O}\right), 1.41\left(\mathrm{~s}, 3 \mathrm{H}, \mathrm{H}-\mathrm{OC}\left(\mathrm{CH}_{3}\right)_{2} \mathrm{O}\right), 1.20\left(\mathrm{~d}, 3 \mathrm{H}, \mathrm{H}-8, J_{8,2}=7.3 \mathrm{~Hz}\right)$.

\section{(3aS,5R,6S,13S,13aR)-5-Methoxymethoxy-2,2,6,12-tetramethyl-13-triisopropylsilyloxydecahydro-7H-}

\section{[1,3]dioxolo[4,5-f][1] oxacyclododecin-7-one (S12)}

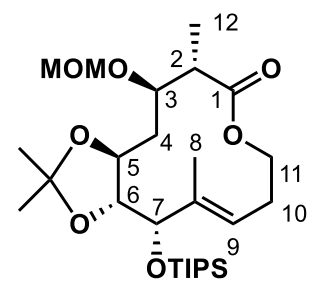

A solution of $\mathrm{Ni}(\mathrm{cod})_{2}(9.4 \mathrm{mg}, 35 \mu \mathrm{mol})$, IMes $\cdot \mathrm{HCl}(12 \mathrm{mg}, 35 \mu \mathrm{mol})$ and ${ }^{t} \mathrm{BuOK}(3$ $\mathrm{mg}, 18 \mu \mathrm{mol})$ in THF $(10 \mathrm{~mL})$ was stirred at room temperature for $10 \mathrm{~min}$. The prepared Niligand solution $(1.6 \mathrm{~mL}, 65 \mu \mathrm{mol})$ was added to a solution of the crude $\mathbf{S 1 1}$ and ${ }^{i} \mathrm{Pr}_{3} \mathrm{SiH}$ (28 $\mu \mathrm{L}, 138 \mu \mathrm{mol})$ in THF $(3.8 \mathrm{~mL})$ at room temperature for $16 \mathrm{~h}$. The reaction was diluted with hexane and filtered through a short silica gel column. The filtrate was concentrated in vacuo.

The residue was semi-purified by flash silica gel column chromatography $(\phi 1.5 \times 10 \mathrm{~cm}, 10 \%$ EtOAc/hexane $)$ to afford a crude $\mathbf{S 1 2}(4.9 \mathrm{mg})$. The crude $\mathbf{S 1 2}$ was used for the next reaction without further purification. ${ }^{1} \mathrm{H}$ NMR $\left(\mathrm{CDCl}_{3}, 400 \mathrm{MHz}\right) \delta 5.63\left(\mathrm{~d}, 1 \mathrm{H}, \mathrm{H}-9, J_{9,10}=11.5 \mathrm{~Hz}\right), 4.91\left(\mathrm{~d}, 1 \mathrm{H}, \mathrm{H}-\mathrm{OCH} \mathrm{H}_{2} \mathrm{O}, J_{\text {gem }}=6.9 \mathrm{~Hz}\right), 4.68$ (ddd, $1 \mathrm{H}, \mathrm{H}-11$, $\left.J_{g e m}=J_{11,10}=12.0, J_{11,10}=2.8 \mathrm{~Hz}\right), 4.58\left(\mathrm{~d}, 1 \mathrm{H}, \mathrm{H}-\mathrm{OCH}_{2} \mathrm{O}, J_{\text {gem }}=6.4 \mathrm{~Hz}\right), 4.43(\mathrm{br} \mathrm{s}, 1 \mathrm{H}, \mathrm{H}-7), 3.95(\mathrm{~m}, 2 \mathrm{H}, \mathrm{H}-5, \mathrm{H}-$ 11), $3.70\left(\mathrm{ddd}, 1 \mathrm{H}, \mathrm{H}-3, J_{3,2}=J_{g e m}=11.0, J_{3,4}=4.1 \mathrm{~Hz}\right), 3.62\left(\mathrm{dd}, 1 \mathrm{H}, \mathrm{H}-6, J_{6,5}=8.2, J_{6,7}=2.3 \mathrm{~Hz}\right), 3.39(\mathrm{~s}, 3 \mathrm{H}, \mathrm{H}-$ $\left.\mathrm{OCH}_{2} \mathrm{OCH}_{3}\right), 2.70\left(\mathrm{dddd}, 1 \mathrm{H}, \mathrm{H}-10, J_{\text {gem }}=16.0, J_{10,9}=12.0, J_{10,11}=7.8, J_{10,11}=4.8 \mathrm{~Hz}\right), 2.43\left(\mathrm{dq}, 1 \mathrm{H}, \mathrm{H}-2, J_{2,3}=\right.$ 10.0, $\left.J_{2,12}=6.7 \mathrm{~Hz}\right), 2.08\left(\mathrm{~d}, 1 \mathrm{H}, \mathrm{H}-10, J_{g e m}=16.0 \mathrm{~Hz}\right), 1.65(\mathrm{~m}, 2 \mathrm{H}, \mathrm{H}-4), 1.56(\mathrm{~s}, 3 \mathrm{H}, \mathrm{H}-8), 1.37(\mathrm{~s}, 3 \mathrm{H}, \mathrm{H}-$ $\left.\mathrm{OC}\left(\mathrm{CH}_{3}\right)_{2} \mathrm{O}\right), 1.33\left(\mathrm{~s}, 3 \mathrm{H}, \mathrm{H}-\mathrm{OC}\left(\mathrm{CH}_{3}\right)_{2} \mathrm{O}\right), 1.23\left(\mathrm{~d}, 3 \mathrm{H}, \mathrm{H}-12, J_{12,2}=6.7 \mathrm{~Hz}\right), 1.05\left(\mathrm{~m}, 21 \mathrm{H}, \mathrm{H}-\mathrm{SiCH}\left(\mathrm{CH}_{3}\right)_{2}\right)$.

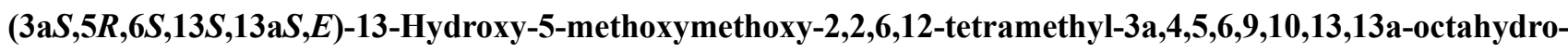
7H-[1,3]dioxolo[4,5-f][1] oxacyclododecin-7-one (37)

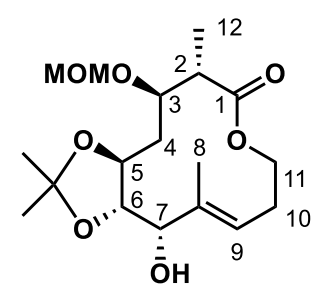

A solution of the crude $\mathbf{S 1 2}(4.9 \mathrm{mg})$ in THF $(0.5 \mathrm{~mL})$ was treated with TBAF $(10 \mu \mathrm{L}$, $10 \mu \mathrm{mol}, 1 \mathrm{M}$ in THF) at room temperature for $3 \mathrm{~h}$. The mixture was partitioned between EtOAc and $\mathrm{H}_{2} \mathrm{O}$. The organic phase was washed with $1 \mathrm{M}$ aq. $\mathrm{HCl}$, sat. aq. $\mathrm{NaHCO}_{3}, \mathrm{H}_{2} \mathrm{O}$ and brine, dried $\left(\mathrm{Na}_{2} \mathrm{SO}_{4}\right)$, filtered and concentrated in vacuo. The residue was purified by hi-flash silica gel column chromatography $(\phi 1.6 \times 6 \mathrm{~cm}, 30 \rightarrow 50 \%$ EtOAc/hexane) to afford 37 (3.6 mg, $95 \mu \mathrm{mol}, 14 \%$ over 3 steps) as a colorless oil. ${ }^{1} \mathrm{H}$ NMR $\left(\mathrm{CDCl}_{3}, 400 \mathrm{MHz}\right) \delta 5.70\left(\mathrm{~d}, 1 \mathrm{H}, \mathrm{H}-9, J_{9,10}=11.9\right.$ $\mathrm{Hz}), 4.91\left(\mathrm{~d}, 1 \mathrm{H}, \mathrm{OCH}_{2} \mathrm{O}, J_{g e m}=6.4 \mathrm{~Hz}\right), 4.80\left(\mathrm{dd}, 1 \mathrm{H}, \mathrm{H}-11, J_{g e m}=J_{11,10}=11.0 \mathrm{~Hz}\right), 4.58\left(\mathrm{~d}, 1 \mathrm{H}, \mathrm{H}-\mathrm{OCH} H_{2} \mathrm{O}, J_{g e m}=\right.$ $6.4 \mathrm{~Hz}$ ), 4.30 (br s, 1H, H-7), 3.94 (dd, 1H, H-11, $\left.J_{g e m}=11.0, J_{11,10}=3.9 \mathrm{~Hz}\right), 3.81$ (ddd, $1 \mathrm{H}, \mathrm{H}-5, J_{5,4}=4.1, J_{5,6}=$ $8.2 \mathrm{~Hz}$ ), 3.74 (m, 1H, H-3), 3.72 (m, 1H, H-6), 3.40 (s, 3H, H-OCH $\mathrm{OCH}_{3}$ ), 2.77 (dddd, 1H, H-10, Jgem = 16.0, J10,9 $\left.=11.9, J_{10,11}=3.9, J_{10,11}=11.0 \mathrm{~Hz}\right), 2.46\left(\mathrm{dq}, 1 \mathrm{H}, \mathrm{H}-2, J_{2,3}=10.5, J_{2,12}=6.9 \mathrm{~Hz}\right), 2.18\left(\mathrm{~d}, 1 \mathrm{H}, \mathrm{H}-10, J_{\text {gem }}=16.0 \mathrm{~Hz}\right)$, 
2.14 (m, 1H, H-OH), 1.67 (m, 2H, H-4), 1.61 (br s, 3H, H-8), 1.43 (s, 3H, H-OC(CH3) $2 \mathrm{O}), 1.40\left(\mathrm{~s}, 3 \mathrm{H}, \mathrm{H}-\mathrm{OC}(\mathrm{CH})_{2} \mathrm{O}\right)$, $1.25\left(\mathrm{~d}, 3 \mathrm{H}, \mathrm{H}-12, J_{12,2}=6.9 \mathrm{~Hz}\right) ;{ }^{13} \mathrm{C} \mathrm{NMR}\left(\mathrm{CDCl}_{3}, 100 \mathrm{MHz}\right) \delta 174.5,132.9,122.1,109.1,95.3,80.5,74.4,71.4$, 62.9, 56.4, 47.8, 42.0, 28.4, 27.6, 27.2, 16.0, 15.1, 0.15; ESIMS-LR m/z 381 [(M+Na) $)^{+}$; HRMS (ESI) m/z: [(M+Na) $\left.{ }^{+}\right]$ Calcd for $\mathrm{C}_{18} \mathrm{H}_{30} \mathrm{O}_{7} \mathrm{Na} 381.1889$; Found 381.1902; $[\alpha]^{20}{ }_{\mathrm{D}}-127.6\left(c\right.$ 0.30, $\left.\mathrm{CHCl}_{3}\right)$.

Scheme S6. Synthesis of $\mathbf{3 9}$ and S17

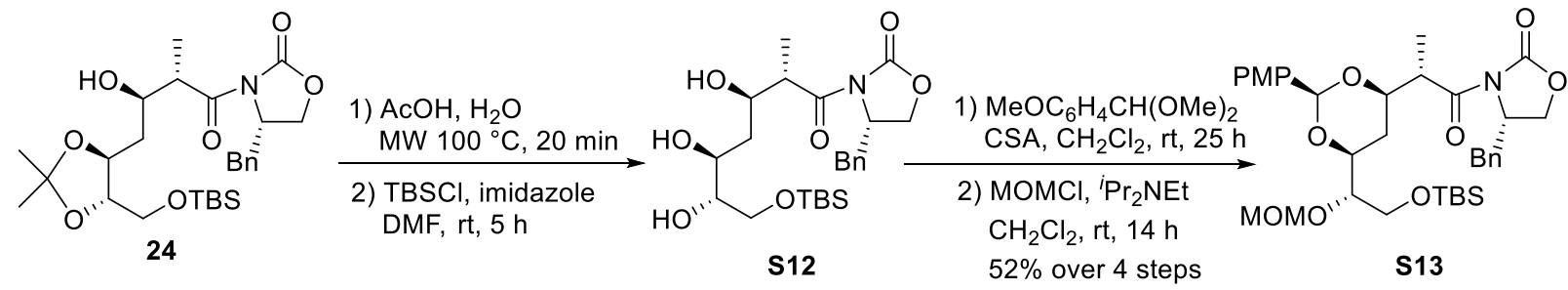

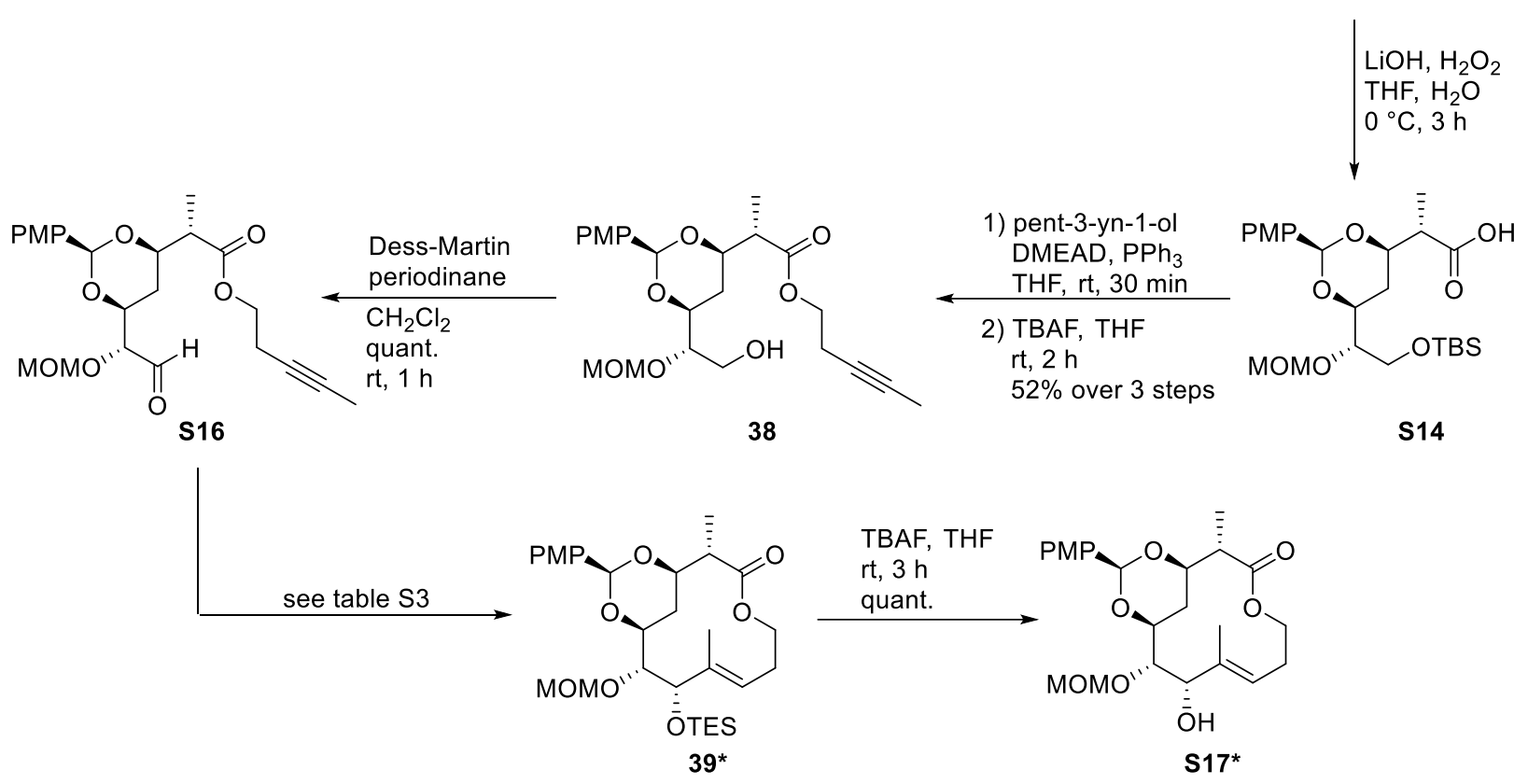

\begin{tabular}{|c|c|c|c|c|c|c|c|}
\hline entry & $\begin{array}{l}\mathrm{Ni(cod})_{2} \\
(\mathrm{~mol} \%)\end{array}$ & $\begin{array}{l}\text { IMes } \cdot \mathrm{HCl} \\
(\mathrm{mol} \%)\end{array}$ & $\begin{array}{l}{ }^{t} \mathrm{BuOK} \\
(\mathrm{mol} \%)\end{array}$ & $\begin{array}{l}\text { reductant } \\
\text { (eq.) }\end{array}$ & temp. & solvent & yield $(\%)$ \\
\hline 1 & 50 & 50 & 50 & $\mathrm{Et}_{3} \mathrm{SiH}(2)$ & rt & $\begin{array}{l}\text { THF } \\
(5 \mathrm{mM})\end{array}$ & - \\
\hline 2 & 50 & 50 & 50 & $\mathrm{Et}_{3} \mathrm{SiH}(2)$ & $\mathrm{rt}$ & $\begin{array}{l}\text { toluene } \\
(5 \mathrm{mM})\end{array}$ & 21 \\
\hline 3 & 50 & 50 & 50 & $\mathrm{Et}_{3} \mathrm{SiH}(2)$ & $60^{\circ} \mathrm{C}$ & $\begin{array}{l}\text { toluene } \\
(5 \mathrm{mM})\end{array}$ & 46 \\
\hline 4 & 50 & 50 & 50 & $\mathrm{Et}_{3} \mathrm{SiH}(2)$ & $70^{\circ} \mathrm{C}$ & $\begin{array}{l}\text { toluene } \\
(5 \mathrm{mM})\end{array}$ & 61 \\
\hline
\end{tabular}




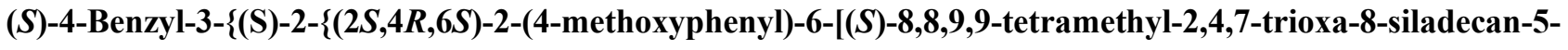

\section{yl]-1,3-dioxan-4-yl\}propanoyl\}oxazolidin-2-one (S13)}

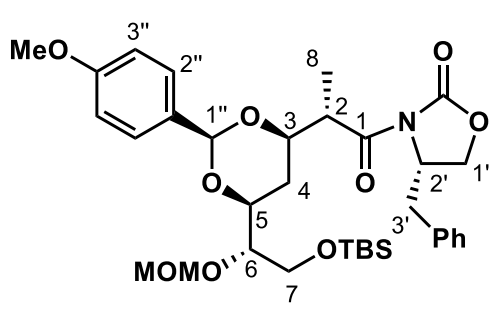

A solution of $24(500 \mathrm{mg}, 0.96 \mathrm{mmol})$ in $\mathrm{AcOH}(1 \mathrm{~mL})$ and $\mathrm{H}_{2} \mathrm{O}(3 \mathrm{~mL})$ was stirred at $100{ }^{\circ} \mathrm{C}$ under microwave irradiation for $20 \mathrm{~min}(0 \mathrm{bar}, 10 \mathrm{~W})$. The mixture was concentrated in vacuo. A solution of the residue in DMF $(5 \mathrm{~mL})$ was treated with imidazole $(170 \mathrm{mg}, 2.5 \mathrm{mmol})$ and $\mathrm{TBSCl}(158 \mathrm{mg}, 1.05 \mathrm{mmol})$ at room temperature for $5 \mathrm{~h}$. The reaction was quenched with $\mathrm{MeOH}$. The mixture was partitioned between EtOAc and $1 \mathrm{M}$ aq. $\mathrm{HCl}$. The organic phase was washed with sat. aq. $\mathrm{NaHCO}_{3}, \mathrm{H}_{2} \mathrm{O}$ and brine, dried $\left(\mathrm{Na}_{2} \mathrm{SO}_{4}\right)$, filtered and concentrated in vacuo. A solution of the residue in $\mathrm{CH}_{2} \mathrm{Cl}_{2}(10 \mathrm{~mL})$ was treated with CSA (23 mg, $0.1 \mathrm{mmol})$ and $p$-anisaldehyde dimethyl acetal $(511 \mu \mathrm{L}, 3.0 \mathrm{mmol})$ at room temperature for $25 \mathrm{~h}$. The mixture was partitioned between EtOAc and $\mathrm{H}_{2} \mathrm{O}$. The organic phase was washed with $1 \mathrm{M} a q$. $\mathrm{HCl}$, sat. aq. $\mathrm{NaHCO}_{3}, \mathrm{H}_{2} \mathrm{O}$ and brine, dried $\left(\mathrm{Na}_{2} \mathrm{SO}_{4}\right)$, filtered and concentrated in vacuo. A solution of the residue in $\mathrm{CH}_{2} \mathrm{Cl}_{2}(10$ $\mathrm{mL}$ ) was treated with ${ }^{i} \operatorname{Pr}_{2} \mathrm{NEt}(697 \mu \mathrm{L}, 4 \mathrm{mmol})$ and $\mathrm{MOMCl}(150 \mu \mathrm{L}, 2 \mathrm{mmol})$ at room temperature for $14 \mathrm{~h}$. The reaction was quenched with $\mathrm{H}_{2} \mathrm{O}$. The mixture was partitioned between EtOAc and $\mathrm{H}_{2} \mathrm{O}$. The organic phase was washed with $1 \mathrm{M}$ aq. $\mathrm{HCl}$, sat. aq. $\mathrm{NaHCO}_{3}, \mathrm{H}_{2} \mathrm{O}$ and brine, dried $\left(\mathrm{Na}_{2} \mathrm{SO}_{4}\right)$, filtered and concentrated in vacuo. The residue was purified by silica gel column chromatography $(\phi 4 \times 10 \mathrm{~cm}, 17 \%$ EtOAc/hexane) to afford $\mathbf{S 1 3}(318 \mathrm{mg}$, $0.494 \mathrm{mmol}, 52 \%$ over 4 steps $)$ as a colorless oil. ${ }^{1} \mathrm{H} \mathrm{NMR}\left(\mathrm{CDCl}_{3}, 400 \mathrm{MHz}\right) \delta 7.38\left(\mathrm{~d}, 2 \mathrm{H}, \mathrm{H}-2 ", J_{2 ", 3 "}=6.0 \mathrm{~Hz}\right)$, 7.26 (m, 5H, H-Ph), 6.85 (d, 2H, H-3", $J_{3^{\prime \prime}, 2^{\prime \prime}}=6.0 \mathrm{~Hz}$ ), 5.50 (s, 1H, H-1"), 4.80 (d, 1H, H-OCH $\left.\mathrm{H}_{2} \mathrm{O}, J_{\text {gem }}=6.0 \mathrm{~Hz}\right)$, $4.72\left(\mathrm{~d}, 1 \mathrm{H}, \mathrm{H}-\mathrm{OCH} \mathrm{H}_{2} \mathrm{O}, J_{\text {gem }}=6.0 \mathrm{~Hz}\right), 4.65$ (m, 1H, H-2'), 4.11 (m, 4H, H-3, H-5, H-1'), 3.83 (m, 2H, H-2, H-6), 3.79 (s, $\left.3 \mathrm{H}, \mathrm{H}-\mathrm{C}_{6} \mathrm{H}_{4} \mathrm{OCH} H_{3}\right), 3.70$ (m, 2H, H-7), 3.38 (s, 3H, H-OCH $\left.\mathrm{OCH}_{3}\right), 3.25$ (d, 1 H, H-3', $\left.J_{g e m}=13.3 \mathrm{~Hz}\right), 2.76$ $\left(\mathrm{dd}, 1 \mathrm{H}, \mathrm{H}-3^{\prime}, J_{3^{\prime}, 2^{\prime}}=J_{\text {gem }}=13.3 \mathrm{~Hz}\right), 1.68(\mathrm{~m}, 2 \mathrm{H}, \mathrm{H}-4), 1.35(\mathrm{~m}, 3 \mathrm{H}, \mathrm{H}-8), 0.88\left(\mathrm{~s}, 9 \mathrm{H}, \mathrm{H}-{ }^{t} \mathrm{Bu}\right), 0.05(\mathrm{~s}, 6 \mathrm{H}, \mathrm{H}-$ $\left.\mathrm{SiCH}_{3}\right) ;{ }^{13} \mathrm{C} \mathrm{NMR}\left(\mathrm{CDCl}_{3}, 100 \mathrm{MHz}\right) \delta 174.6,156.0,129.7,129.1,127.4,113.5,104.4,100.6,97.1,66.0,56.0,55.6$, 55.3, 52.5, 42.3, 38.0, 26.0, 13.4, 6.61, 0.15, -5.3; ESIMS-LR m/z $666\left[(\mathrm{M}+\mathrm{Na})^{+}\right]$; HRMS (ESI) m/z: [(M+Na) $\left.{ }^{+}\right]$ Calcd for $\mathrm{C}_{34} \mathrm{H}_{49} \mathrm{NO}_{9} \mathrm{NaSi} 666.3074$; Found 666.3090; $[\alpha]^{20}{ }_{\mathrm{D}}+48.8\left(c 0.14, \mathrm{CHCl}_{3}\right)$.

Pent-3-yn-1-yl (S)-2-\{(2S,4R,6S)-6-[(S)-2-hydroxy-1-methoxymethoxyethyl]-2-(4-methoxyphenyl)-1,3-dioxan4-yl\}propanoate (38)

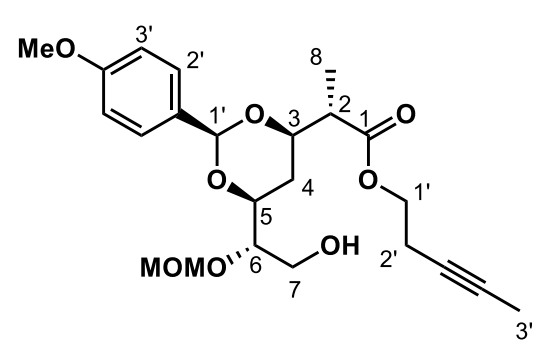

A solution of $\mathbf{S 1 3}(68 \mathrm{mg}, 88 \mu \mathrm{mol})$ in THF $(0.8 \mathrm{~mL})$ and $\mathrm{H}_{2} \mathrm{O}(0.2 \mathrm{~mL})$ was treated with $\mathrm{H}_{2} \mathrm{O}_{2}\left(80 \mu \mathrm{L}, 0.70 \mathrm{mmol}, 30 \%\right.$ in $\left.\mathrm{H}_{2} \mathrm{O}\right)$ and $\mathrm{LiOH}(15 \mathrm{mg}$, $0.35 \mathrm{mmol}$ ) at $0{ }^{\circ} \mathrm{C}$ for $3 \mathrm{~h}$. The reaction was quenched with sat. aq. $\mathrm{Na}_{2} \mathrm{~S}_{2} \mathrm{O}_{3}$. The mixture was partitioned between $\mathrm{Et}_{2} \mathrm{O}$ and sat. aq. $\mathrm{NaHCO}_{3}$. The organic phase was washed with brine, dried $\left(\mathrm{Na}_{2} \mathrm{SO}_{4}\right)$, filtered and concentrated in vacuo to give S14. A solution of the residue and pent-3-yn-1-ol $(24 \mu \mathrm{L}, 0.26$ 
mmol) in THF $(1 \mathrm{~mL})$ was treated with $\mathrm{PPh}_{3}(69 \mathrm{mg}, 0.26 \mathrm{mmol})$ and DMEAD $(69 \mathrm{mg}, 0.26 \mathrm{mmol})$ at room temperature for $30 \mathrm{~min}$. The mixture was concentrated in vacuo. The residue was purified by silica gel column chromatography $(\phi 2 \times 7 \mathrm{~cm}, 17 \%$ EtOAc/hexane) to afford a crude ester. A solution of the crude ester in THF (1 $\mathrm{mL})$ was treated with TBAF $(132 \mu \mathrm{L}, 132 \mu \mathrm{mol}, 1 \mathrm{M}$ in THF) at room temperature for $2 \mathrm{~h}$. The mixture was partitioned between EtOAc and $\mathrm{H}_{2} \mathrm{O}$. The organic phase was washed with $1 \mathrm{M} a q$. $\mathrm{HCl}$, sat. aq. $\mathrm{NaHCO}_{3}, \mathrm{H}_{2} \mathrm{O}$ and brine, dried $\left(\mathrm{Na}_{2} \mathrm{SO}_{4}\right)$, filtered and concentrated in vacuo. The residue was purified by hi-flash silica gel column chromatography ( $\phi 4 \times 12 \mathrm{~cm}, 50 \%$ EtOAc/hexane) to afford 38 (20 mg, $46 \mu \mathrm{mol}, 52 \%$ over 3 steps) as a colorless oil. ${ }^{1} \mathrm{H}$ NMR $\left(\mathrm{CDCl}_{3}, 400 \mathrm{MHz}\right) \delta 7.37$ (d, 2H, H-2', $\left.J_{2^{\prime}, 3^{\prime}}=8.7 \mathrm{~Hz}\right), 6.87$ (d, 2H, H-3', $\left.J_{3^{\prime}, 2^{\prime}}=8.7 \mathrm{~Hz}\right), 5.50$ (s, 1H, H$\left.1^{\prime}\right), 4.80\left(\mathrm{~d}, 1 \mathrm{H}, \mathrm{H}-\mathrm{OCH}_{2} \mathrm{O}, J_{\text {gem }}=6.9 \mathrm{~Hz}\right), 4.75\left(\mathrm{~d}, 1 \mathrm{H}, \mathrm{H}-\mathrm{OCH}_{2} \mathrm{O}, J_{\text {gem }}=6.9 \mathrm{~Hz}\right), 4.17$ (t, 2H, H-1', $\left.J_{1^{\prime}, 2^{\prime}}=6.0 \mathrm{~Hz}\right)$, 4.05 (m, 2H, H-3, H-5), 3.81 (m, 1H, H-6), 3.80 (s, 3H, H-C $\left.\mathrm{C}_{4} \mathrm{OCH}_{3}\right), 3.69$ (m, 2H, H-7), 3.43 (s, 3H, H$\left.\mathrm{OCH}_{2} \mathrm{OCH} \mathrm{H}_{3}\right), 2.70$ (m, 2H, H-2, H-OH), 2.47 (m, 2H, H-2'), 1.76 (t, 3H, H-1', $\left.J_{1^{\prime}, 2^{\prime}}=6.0 \mathrm{~Hz}\right), 1.65$ (m, 2H, H-4), 1.30 (d, 3H, H-8, $\left.J_{8,2}=7.4 \mathrm{~Hz}\right) ;{ }^{13} \mathrm{C} \mathrm{NMR}\left(\mathrm{CDCl}_{3}, 100 \mathrm{MHz}\right) \delta 173.9,160.0,151.2,130.9,127.4,113.6,100.8,97.6,81.7$, 74.8, 63.0, 62.4, 56.1, 55.4, 45.3, 30.1, 19.4, 13.3, 6.62, 3.61, 0.14; ESIMS-LR $m / z 459$ [(M+Na) $]$; HRMS (ESI) $\mathrm{m} / \mathrm{z}:\left[(\mathrm{M}+\mathrm{Na})^{+}\right]$Calcd for $\mathrm{C}_{23} \mathrm{H}_{32} \mathrm{O}_{8} \mathrm{Na} 459.1995$; Found 459.1988; $[\alpha]^{20}{ }_{\mathrm{D}}-10.9\left(c 0.12, \mathrm{CHCl}_{3}\right)$.

\section{Pent-3-yn-1-yl (S)-2-\{(2S,4R,6S)-6-[(R)-1-methoxymethoxy-2-oxoethyl]-2-(4-methoxyphenyl)-1,3-dioxan-4-} yl\}propanoate (S16)

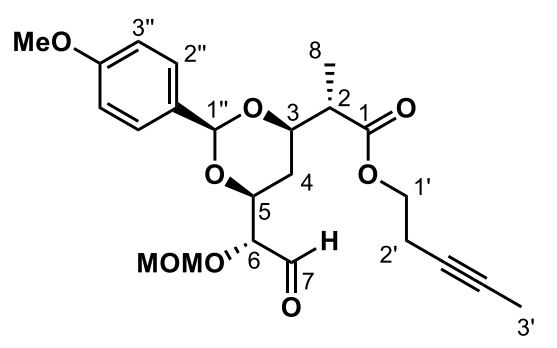

A solution of $38(57 \mathrm{mg}, 0.13 \mathrm{mmol})$ in $\mathrm{CH}_{2} \mathrm{Cl}_{2}(2 \mathrm{~mL})$ was treated with Dess-Martin periodinane $(110 \mathrm{mg}, 0.26 \mathrm{mmol})$ at room temperature for $1 \mathrm{~h}$. Ethyl acetate, sat.aq. $\mathrm{NaHCO}_{3}$ and sat. aq. $\mathrm{Na}_{2} \mathrm{~S}_{2} \mathrm{O}_{3}$ were added to the mixture, which was stirred for further $10 \mathrm{~min}$. The organic phase was washed with brine, dried $\left(\mathrm{Na}_{2} \mathrm{SO}_{4}\right)$, filtered and concentrated in vacuo to afford a crude $\mathbf{S 1 6}$ (57 $\mathrm{mg})$. The crude $\mathbf{S 1 6}$ was used for the next reaction without further purification. ${ }^{1} \mathrm{H}$ NMR $\left(\mathrm{CDCl}_{3}, 400 \mathrm{MHz}\right) \delta 9.78$ (s, 1H, CHO), 7.36 (d, 2H, H-2", $\left.J_{2^{\prime \prime}, 3^{\prime \prime}}=8.7 \mathrm{~Hz}\right), 6.85$ (d, 2H, H-3", $J_{3^{\prime \prime 2}} 2^{\prime \prime}=8.7 \mathrm{~Hz}$ ), 5.49 (s, 1H, H-1"), 4.76 (d, 1H, H-1', $\left.J_{\text {gem }}=6.8 \mathrm{~Hz}\right), 4.73\left(\mathrm{~d}, 1 \mathrm{H}, \mathrm{H}-1\right.$ ', $\left.J_{\text {gem }}=6.8 \mathrm{~Hz}\right), 4.32\left(\mathrm{ddd}, 1 \mathrm{H}, \mathrm{H}-5, J_{5,4}=11.0, J_{5,4}=2.7, J_{5,6}=4.6 \mathrm{~Hz}\right), 4.16$ (t, 2H, H-11, $\left.J_{11,12}=6.8 \mathrm{~Hz}\right), 4.05$ (m, 2H, H-3, H-6), 3.80 (s, 3H, $\left.\mathrm{H}_{-} \mathrm{C}_{6} \mathrm{H}_{4} \mathrm{OCH}_{3}\right), 3.42\left(\mathrm{~s}, 3 \mathrm{H}, \mathrm{H}-\mathrm{OCH}_{2} \mathrm{OCH}_{3}\right), 2.66$ $\left(\mathrm{dq}, 1 \mathrm{H}, \mathrm{H}-2, J_{2,3}=J_{2,8}=7.3 \mathrm{~Hz}\right), 2.45\left(\mathrm{~m}, 2 \mathrm{H}, \mathrm{H}-2^{\prime}\right), 1.79(\mathrm{~m}, 1 \mathrm{H}, \mathrm{H}-4), 1.76\left(\mathrm{t}, 3 \mathrm{H}, \mathrm{H}-3^{\prime}, J_{3^{\prime}, 2^{\prime}}=2.8 \mathrm{~Hz}\right), 1.65$ (dt, $\left.1 \mathrm{H}, \mathrm{H}-4, J_{g e m}=13.0, J_{4,5}=2.7, J_{4,3}=2.3 \mathrm{~Hz}\right), 1.30\left(\mathrm{~d}, 3 \mathrm{H}, \mathrm{H}-8, J_{8,2}=7.4 \mathrm{~Hz}\right)$. 


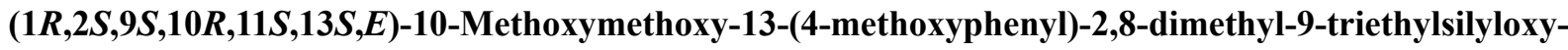
4,12,14-trioxabicyclo[9.3.1]pentadec-7-en-3-one (39)

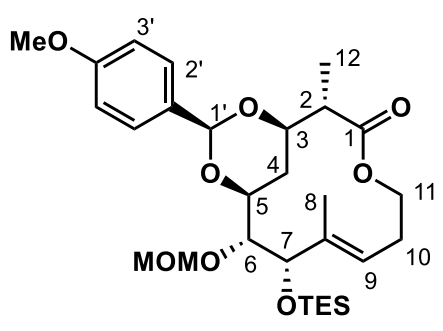

A solution of $\mathrm{Ni}(\operatorname{cod})_{2}(55 \mathrm{mg}, 0.2 \mathrm{mmol}), \mathrm{IMes} \cdot \mathrm{HCl}(68 \mathrm{mg}, 0.2 \mathrm{mmol})$ and ${ }^{t} \mathrm{BuOK}(22 \mathrm{mg}, 0.2 \mathrm{mmol})$ in toluene $(5 \mathrm{~mL})$ was stirred at room temperature for 10 $\min$. The prepared Ni-ligand solution $(1.6 \mathrm{~mL}, 65 \mu \mathrm{mol})$ was added to a solution of the crude $\mathbf{S 1 6}$ and $\mathrm{Et}_{3} \mathrm{SiH}(42 \mu \mathrm{L}, 0.26 \mathrm{mmol})$ in toluene $(24 \mathrm{~mL})$ at $70{ }^{\circ} \mathrm{C}$ for $1 \mathrm{~h}$. The mixture was diluted with hexane, filtered through a short silica gel and concentrated in vacuo. The residue was purified by flash silica gel column chromatography $(\phi 2 \times 10 \mathrm{~cm}, 10 \%$ EtOAc/hexane) to afford 39 (44 mg, $79 \mu \mathrm{mol}, 61 \%$ over 2 steps) as a colorless oil. ${ }^{1} \mathrm{H}$ NMR $\left(\mathrm{CDCl}_{3}, 400 \mathrm{MHz}\right) \delta$ $7.38\left(\mathrm{~d}, 2 \mathrm{H}, \mathrm{H}-2^{\prime}, J_{2^{\prime}, 3^{\prime}}=8.7 \mathrm{~Hz}\right), 6.85$ (d, 2H, H-3', $\left.J_{3^{\prime}, 2^{\prime}}=8.7 \mathrm{~Hz}\right), 5.43(\mathrm{~m}, 1 \mathrm{H}, \mathrm{H}-9), 5.41\left(\mathrm{~m}, 1 \mathrm{H}, \mathrm{H}-1^{\prime}\right), 4.91(\mathrm{~m}$, 1H, H-11), 4.86 (d, 1H, H-OCH $\left.H_{2} \mathrm{O}, J_{\text {gem }}=7.3 \mathrm{~Hz}\right), 4.73$ (d, 1H, H-OCH $\mathrm{H}_{2} \mathrm{O}, J_{\text {gem }}=7.3 \mathrm{~Hz}$ ), 4.48 (br s, 1H, H-7), 4.31 (m, 1H, H-11), 4.11 (m, 1H, H-5), 3.90 (m, 1H, H-6), 3.79 (s, 3H, H-C $\left.\mathrm{H}_{4} \mathrm{OCH}_{3}\right), 3.55$ (m, 1H, H-3), 3.40 (s, 3H, H$\left.\mathrm{OCH}_{2} \mathrm{OCH}_{3}\right), 2.80$ (m, 1H, H-10), 2.51 (m, 1H, H-2), 2.18 (m, 1H, H-10), 1.87 (d, 1H, H-4, Jgem $\left.=13.2 \mathrm{~Hz}\right), 1.80(\mathrm{~s}$, $3 \mathrm{H}, \mathrm{H}-8), 1.25$ (m, 3H, H-12), 1.23 (m, 1H, H-4), 0.90 (m, 9H, H- $\left.\mathrm{SiCH}_{2} \mathrm{CH}_{3}\right), 0.64$ (m, 6H, H-SiCH $\left.\mathrm{CH}_{3}\right) ;{ }^{13} \mathrm{C} \mathrm{NMR}$ $\left(\mathrm{CDCl}_{3}, 100 \mathrm{MHz}\right) \delta 174.2,159.8,136.5,129.2,127.7,113.5,112.4,100.6,98.9,55.4,29.7,21.2,18.1,7.1,5.0$, 0.08; ESIMS-LR m/z 573 [(M+Na) $)^{+}$; HRMS (ESI) m/z: $\left[(\mathrm{M}+\mathrm{Na})^{+}\right]$Calcd for $\mathrm{C}_{29} \mathrm{H}_{46} \mathrm{O}_{8} \mathrm{NaSi}$ 573.2860; Found 573.2876; $[\alpha]^{20}{ }_{\mathrm{D}}+20.3\left(c 0.62, \mathrm{CHCl}_{3}\right)$. Conformer of 39 was detected by ${ }^{1} \mathrm{H} \mathrm{NMR}$ analysis and conformational search using MacroModel (Figure S1).

\section{$(1 R, 2 S, 9 S, 10 S, 11 S, 13 S, E)-9-H y d r o x y-10-m e t h o x y m e t h o x y-13-(4-m e t h o x y p h e n y l)-2,8-d i m e t h y l-4,12,14-$} trioxabicyclo[9.3.1]pentadec-7-en-3-one (S17)

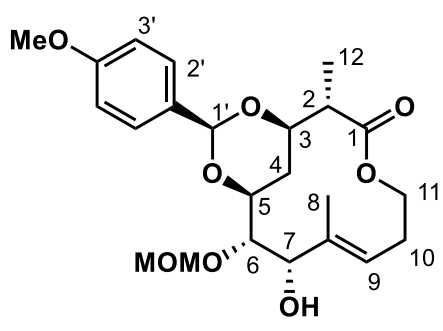

A solution of $39(5 \mathrm{mg}, 9.1 \mu \mathrm{mol})$ in THF $(0.5 \mathrm{~mL})$ was treated with TBAF $(18$ $\mu \mathrm{L}, 18 \mu \mathrm{mol}, 1 \mathrm{M}$ in THF) at room temperature for $3 \mathrm{~h}$. The mixture was partitioned between EtOAc and $\mathrm{H}_{2} \mathrm{O}$. The organic phase was washed with $1 \mathrm{M}$ aq. $\mathrm{HCl}$, sat. aq. $\mathrm{NaHCO}_{3}, \mathrm{H}_{2} \mathrm{O}$ and brine, dried $\left(\mathrm{Na}_{2} \mathrm{SO}_{4}\right)$, filtered and concentrated in vacuo. The residue was purified by flash silica gel column chromatography $(\phi 1 \times 5 \mathrm{~cm}, 20 \%$ EtOAc/hexane) to afford $\mathbf{S 1 7}(4.0 \mathrm{mg}, 8.7 \mu \mathrm{mol}, 96 \%)$ as a colorless oil. ${ }^{1} \mathrm{H} \mathrm{NMR}\left(\mathrm{CDCl}_{3}, 400 \mathrm{MHz}, 25{ }^{\circ} \mathrm{C}\right) \delta 7.35$ $\left(\mathrm{d}, 2 \mathrm{H}, \mathrm{H}-2^{\prime}, J_{2^{\prime}, 3^{\prime}}=8.3 \mathrm{~Hz}\right), 6.87$ (d, 2H, H-3', $\left.J_{3^{\prime}, 2^{\prime}}=8.3 \mathrm{~Hz}\right), 5.49$ (m, 1H, H-9), 5.39 (br s, 1H, H-1'), 4.79 (d, 2H, $\mathrm{H}-\mathrm{OCH}_{2} \mathrm{O}, J_{\text {gem }}=6.4 \mathrm{~Hz}$ ), $4.40(\mathrm{~s}, 1 \mathrm{H}, \mathrm{H}-7), 4.18(\mathrm{~m}, 1 \mathrm{H}, \mathrm{H}-5), 3.97$ (m, 1H, H-6), 3.79 (s, 3H, H-C $\left.6 \mathrm{H}_{4} \mathrm{OCH}\right)_{3}, 3.37$

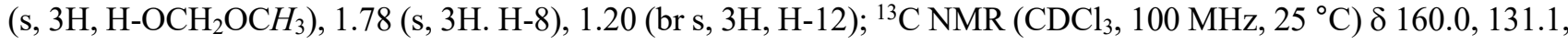
127.5, 113.7, 100.2, 65.3, 56.2, 55.4, 29.9, 23.2, 14.2, 6.5 0.14; ESIMS-LR m/z 459 [(M+Na) $\left.{ }^{+}\right]$; HRMS (ESI) m/z: $\left[(\mathrm{M}+\mathrm{Na})^{+}\right]$Calcd for $\mathrm{C}_{23} \mathrm{H}_{32} \mathrm{O}_{8} \mathrm{Na} 459.1995$; Found 459.1988; $[\alpha]^{20}{ }_{D}-7.3\left(c 1.10, \mathrm{CHCl}_{3}\right)$. Conformer of $\mathbf{S 1 7}$ was detected by variable temperature ${ }^{1} \mathrm{H}$ NMR analysis and conformational search using MacroModel (Figure S1, S2). 
Scheme S7. Synthesis of $\mathbf{S 2 0}$
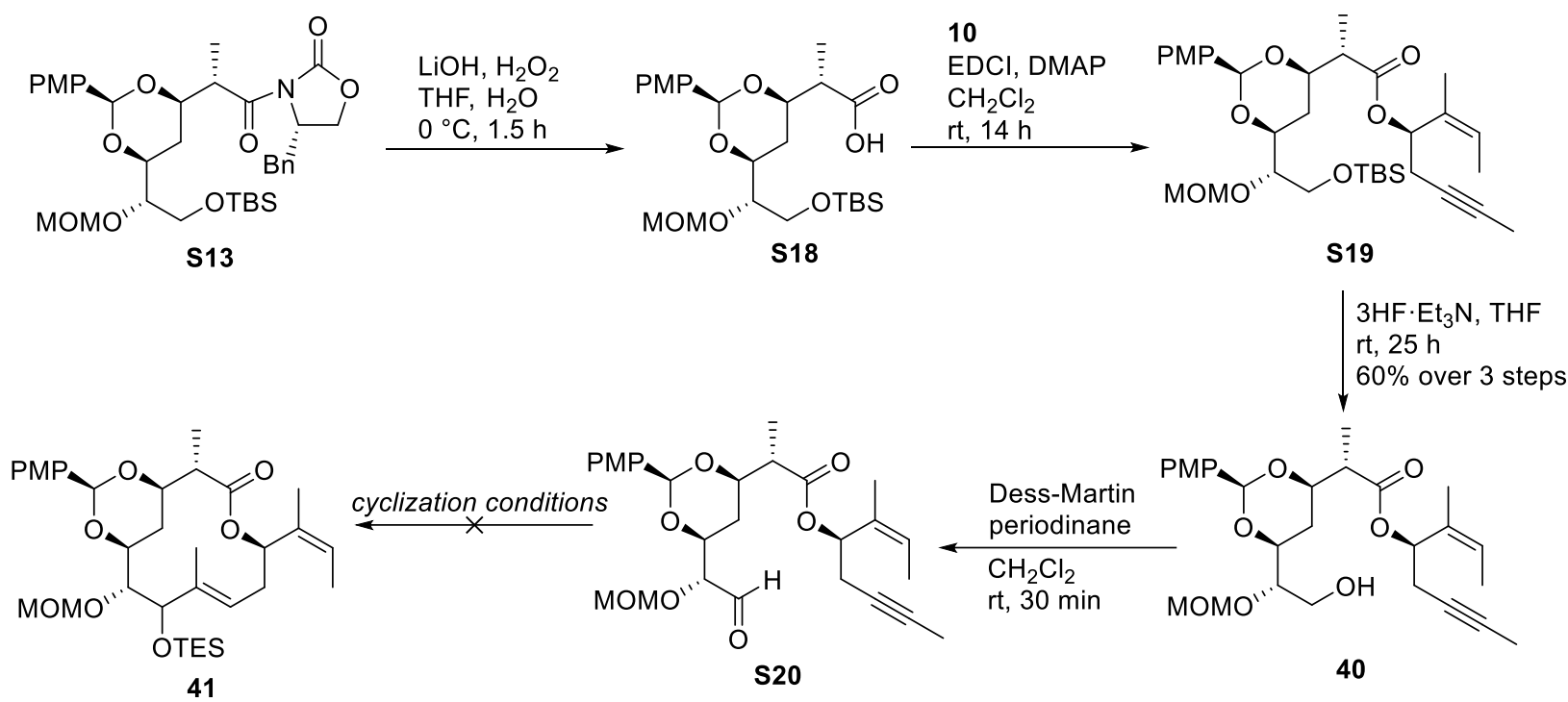

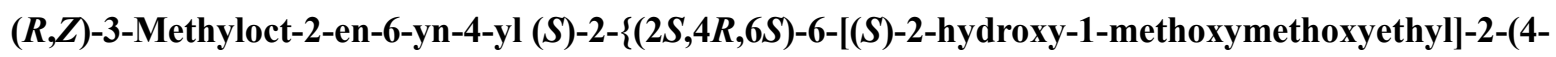
methoxyphenyl)-1,3-dioxan-4-yl\}propanoate (40)

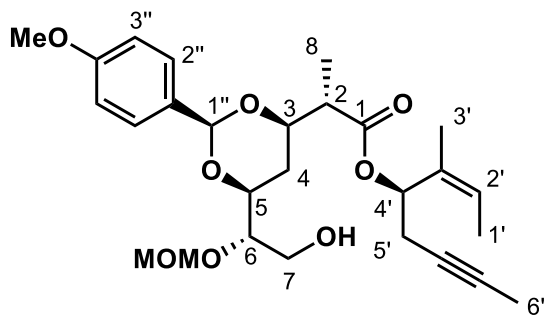

A solution of $\mathbf{S 1 3}(150 \mathrm{mg}, 0.23 \mathrm{mmol})$ in THF $(4 \mathrm{~mL})$ and $\mathrm{H}_{2} \mathrm{O}(1 \mathrm{~mL})$ was treated with $\mathrm{H}_{2} \mathrm{O}_{2}\left(105 \mu \mathrm{L}, 0.92 \mathrm{mmol}, 30 \%\right.$ in $\left.\mathrm{H}_{2} \mathrm{O}\right)$ and $\mathrm{LiOH}(19 \mathrm{mg}$, $0.46 \mathrm{mmol}$ ) at $0{ }^{\circ} \mathrm{C}$ for $1.5 \mathrm{~h}$. The reaction was quenched with sat. aq. $\mathrm{Na}_{2} \mathrm{~S}_{2} \mathrm{O}_{3}$, and the mixture was partitioned between $\mathrm{Et}_{2} \mathrm{O}$ and sat. aq. $\mathrm{NaHCO}_{3}$. The organic phase was washed with brine, dried $\left(\mathrm{Na}_{2} \mathrm{SO}_{4}\right)$, filtered and concentrated in vacuo to give S18. A solution of the residue and $\mathbf{1 0}(100 \mathrm{mg}, 0.23 \mathrm{mmol})$ in $\mathrm{CH}_{2} \mathrm{Cl}_{2}(2 \mathrm{~mL})$ was treated with DMAP $(6 \mathrm{mg}, 46 \mu \mathrm{mol})$ and EDCI $(88 \mathrm{mg}, 0.46 \mathrm{mmol})$ at room temperature for $14 \mathrm{~h}$. The mixture was partitioned between EtOAc and $\mathrm{H}_{2} \mathrm{O}$. The organic phase was washed with $1 \mathrm{M}$ aq. $\mathrm{HCl}$, sat. aq. $\mathrm{NaHCO}_{3}, \mathrm{H}_{2} \mathrm{O}$ and brine, dried $\left(\mathrm{Na}_{2} \mathrm{SO}_{4}\right)$, filtered and concentrated in vacuo to give S19. A solution of the residue in THF (3 mL) was treated with $3 \mathrm{HF} \cdot \mathrm{Et}_{3} \mathrm{~N}(326 \mu \mathrm{L}, 2 \mathrm{mmol})$ at room temperature for $25 \mathrm{~h}$. The mixture was partitioned between EtOAc and $\mathrm{H}_{2} \mathrm{O}$. The organic phase was washed with $1 \mathrm{M}$ aq. $\mathrm{HCl}$, sat. aq. $\mathrm{NaHCO}_{3}, \mathrm{H}_{2} \mathrm{O}$ and brine, dried $\left(\mathrm{Na}_{2} \mathrm{SO}_{4}\right)$, filtered and concentrated in vacuo. The residue was purified by flash silica gel column chromatography $(\phi 1 \times 10 \mathrm{~cm}$, $20 \rightarrow 33 \%$ EtOAc/hexane) to afford 40 (68 mg, $0.14 \mathrm{mmol}, 60 \%$ over 3 steps) as a colorless oil. ${ }^{1} \mathrm{H} \mathrm{NMR}\left(\mathrm{CDCl}_{3}\right.$, $400 \mathrm{MHz}) \delta 7.36$ (d, 2H, H-2", $\left.J_{2^{\prime \prime}, 3^{\prime \prime}}=8.7 \mathrm{~Hz}\right), 6.88$ (d, 2H, H-3", $\left.J_{3^{\prime \prime}, 2^{\prime \prime}}=8.7 \mathrm{~Hz}\right), 5.76$ (t, 1H, H-4', $\left.J_{4^{\prime}, 3^{\prime}}=7.3 \mathrm{~Hz}\right)$, $5.50\left(\mathrm{~s}, 1 \mathrm{H}, \mathrm{H}-1^{\prime}\right), 5.44\left(\mathrm{q}, 1 \mathrm{H}, \mathrm{H}-2^{\prime}, J_{2^{\prime}, 1^{\prime}}=6.8 \mathrm{~Hz}\right), 4.79\left(\mathrm{~d}, 1 \mathrm{H}, \mathrm{H}-\mathrm{OCH} \mathrm{H}_{2} \mathrm{O}, J_{\text {gem }}=6.9 \mathrm{~Hz}\right), 4.75\left(\mathrm{~d}, 1 \mathrm{H}, \mathrm{H}-\mathrm{OCH} \mathrm{H}_{2} \mathrm{O}\right.$, $\left.\left.J_{\text {gem }}=6.9 \mathrm{~Hz}\right), 4.03(\mathrm{~m}, 2 \mathrm{H}, \mathrm{H}-3, \mathrm{H}-5), 3.81(\mathrm{~m}, 1 \mathrm{H}, \mathrm{H}-6), 3.80\left(\mathrm{~s}, 3 \mathrm{H}, \mathrm{H}-\mathrm{C}_{6} \mathrm{H}_{4} \mathrm{OCH}\right)_{3}\right), 3.70\left(\mathrm{~d}, 2 \mathrm{H}, \mathrm{H}-7, J_{7,6}=5.0\right.$ $\mathrm{Hz}$ ), 3.42 (s, 3H, $\mathrm{H}-\mathrm{OCH}_{2} \mathrm{OCH}_{3}$ ), 2.67 (br s, $\left.1 \mathrm{H}, \mathrm{H}-\mathrm{OH}\right), 2.64$ (dq, $1 \mathrm{H}, \mathrm{H}-2, J_{2,3}=J_{2,8}=7.3 \mathrm{~Hz}$ ), 2.53 (ddd, 1H, H$\left.5^{\prime}, J_{\text {gem }}=15.2, J_{5^{\prime}, 4^{\prime}}=7.3, J_{5^{\prime}, 6^{\prime}}=2.8 \mathrm{~Hz}\right), 2.39\left(\mathrm{ddd}, 1 \mathrm{H}, \mathrm{H}-5^{\prime}, J_{g e m}=15.2, J_{5^{\prime}, 4^{\prime}}=7.3, J_{5^{\prime}, 6^{\prime}}=2.3 \mathrm{~Hz}\right), 1.63(\mathrm{~m}, 11 \mathrm{H}, \mathrm{H}-$ 
4, H-1', H-3', H-6'), 1.28 (d, 3H, H-8, $\left.J_{8,2}=7.3 \mathrm{~Hz}\right) ;{ }^{13} \mathrm{C} \mathrm{NMR}\left(\mathrm{CDCl}_{3}, 100 \mathrm{MHz}\right) \delta 173.1,160.0,132.2,130.9,129.2$, 128.8, 127.4, 125.0, 113.6, 100.8, 97.6, 81.7, 74.5, 71.0, 62.4, 56.0, 55.4, 45.4, 41.7, 30.1, 23.1, 17.9, 13.4, 6.6, 3.6, 0.14, ; ESIMS-LR $m / z 513$ [(M+Na) $)^{+}$; HRMS (ESI) m/z: [(M+Na $\left.)^{+}\right]$Calcd for $\mathrm{C}_{27} \mathrm{H}_{38} \mathrm{O}_{8} \mathrm{Na}$ 513.2464; Found $513.2459 ;[\alpha]^{20}{ }_{D}-28.3\left(c 0.10, \mathrm{CHCl}_{3}\right)$.

\section{$(R, Z)-3-M e t h y l o c t-2-e n-6-y n-4-y l(S)-2-\{(2 S, 4 R, 6 S)-6-[(R)-1-m e t h o x y m e t h o x y-2-0 x o e t h y l]-2-(4-$}

\section{methoxyphenyl)-1,3-dioxan-4-yl\}propanoate (S20)}

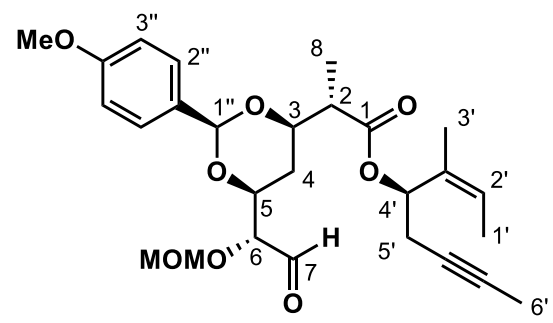

A solution of $40(67 \mathrm{mg}, 0.137 \mathrm{mmol})$ in $\mathrm{CH}_{2} \mathrm{Cl}_{2}(1.5 \mathrm{~mL})$ was treated with Dess-Martin periodinane (116 mg, $0.273 \mathrm{mmol}$ ) at room temperature for 30 min. The reaction was quenched with sat. aq. $\mathrm{Na}_{2} \mathrm{~S}_{2} \mathrm{O}_{3}$ and sat. aq. $\mathrm{NaHCO}_{3}$. The mixture was partitioned between EtOAc and $\mathrm{H}_{2} \mathrm{O}$. The organic phase was washed with brine, dried $\left(\mathrm{Na}_{2} \mathrm{SO}_{4}\right)$, filtered and concentrated in vacuo to afford a crude $\mathbf{S 2 0}(67 \mathrm{mg})$ The crude $\mathbf{S 2 0}$ was used for the next reaction without further purification. ${ }^{1} \mathrm{H}$ $\operatorname{NMR}\left(\mathrm{CDCl}_{3}, 400 \mathrm{MHz}\right) \delta 9.78(\mathrm{~s}, 1 \mathrm{H}, \mathrm{CHO}), 7.35$ (d, 2H, H-2", $\left.J_{2^{\prime \prime}, 3^{\prime \prime}}=8.7 \mathrm{~Hz}\right), 6.85$ (d, 2H, H-3", $\left.J_{3^{\prime \prime}, 2^{2}}=8.7 \mathrm{~Hz}\right)$, $5.76\left(\mathrm{~d}, 1 \mathrm{H}, \mathrm{H}-4^{\prime}, J_{4^{\prime}, 5^{\prime}}=7.3 \mathrm{~Hz}\right), 5.48\left(\mathrm{~s}, 1 \mathrm{H}, \mathrm{H}-1^{\prime \prime}\right), 5.40$ (q, 1H, H-2', $\left.J_{2^{\prime}, 1^{\prime}}=6.9 \mathrm{~Hz}\right), 4.78$ (d, 1H, H-OCH $H_{2} \mathrm{O}, J_{g e m}=$ $6.9 \mathrm{~Hz}$ ), $4.73\left(\mathrm{~d}, 1 \mathrm{H}, \mathrm{H}-\mathrm{OCH} \mathrm{H}_{2} \mathrm{O}, J_{\text {gem }}=6.9 \mathrm{~Hz}\right), 4.31$ (m, 1H, H-5), 4.04 (m, 2H, H-3, H-6), 3.79 (s, 3H, H-C $\left.6 \mathrm{H}_{4} \mathrm{OCH}\right)_{3}$, $3.52\left(\mathrm{~s}, 3 \mathrm{H}, \mathrm{H}-\mathrm{OCH}_{2} \mathrm{OCH}_{3}\right), 2.64$ (dq, $\left.1 \mathrm{H}, \mathrm{H}-2, J_{2,8}=J_{2,3}=7.3 \mathrm{~Hz}\right), 2.52\left(\mathrm{~m}, 1 \mathrm{H}, \mathrm{H}-5^{\prime}\right), 2.40\left(\mathrm{~m}, 1 \mathrm{H}, \mathrm{H}-5^{\prime}\right), 1.78(\mathrm{~m}$, 2H, H-4), 1.72 (s, 3H, H-6'), 1.70 (m, 3H, H-1'), 1.65 (s, 3H, H-3') 1.28 (d, 3H, H-8, J8,2 = 7.3 Hz). 


\section{Scheme S8. Synthesis of $\mathbf{S 3 0}$}

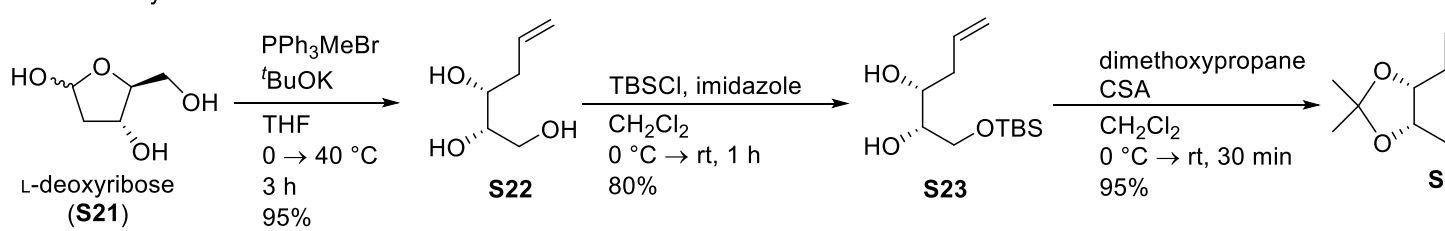

$\mid \begin{aligned} & \mathrm{K}_{2} \mathrm{OsO}_{4} \cdot 2 \mathrm{H}_{2} \mathrm{O} \\ & \mathrm{NalO}_{4} \\ & 2,6 \text {-lutidine } \\ & \text { dioxane, } \mathrm{H}_{2} \mathrm{O} \\ & 0^{\circ} \mathrm{C} \rightarrow \mathrm{rt}, 2 \mathrm{~h}\end{aligned}$

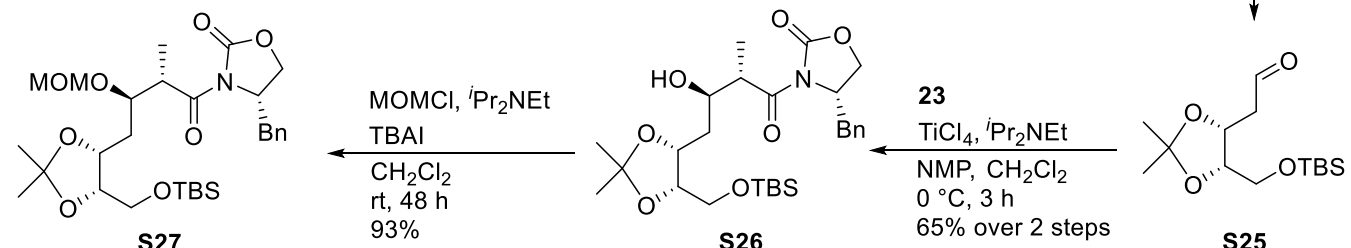<smiles>CO[C@H](C[C@@H]1OC(C)(C)O[C@@H]1C[O+][Sb])[C@@H](OC)C(=O)O</smiles>

S28

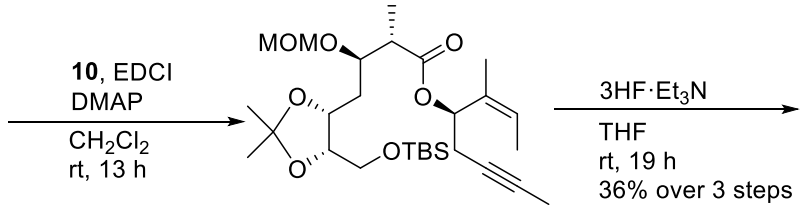

S29

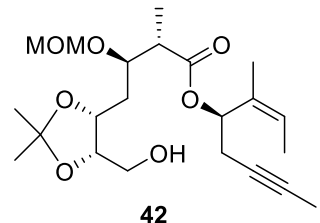

42

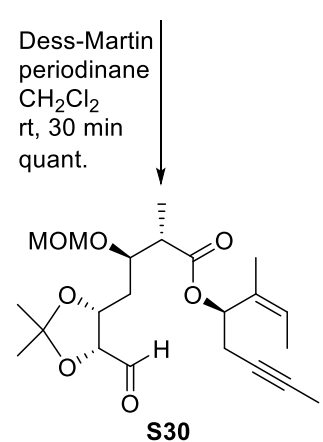

\section{(2S,3R)-Hex-5-ene-1,2,3-triol (S22)}<smiles>CC(C)=CC[C@@H](O)[C@H](O)C(C)O</smiles>

A solution of methyltriphenylphosphonium bromide $(21.7 \mathrm{~g}, 60.8 \mathrm{mmol})$ in THF $(250 \mathrm{~mL})$ was treated with ${ }^{t} \mathrm{BuOK}(6.83 \mathrm{~g}, 60.8 \mathrm{mmol})$ at $0{ }^{\circ} \mathrm{C}$ for $30 \mathrm{~min}$. L-Deoxyribose $(4.08 \mathrm{~g}, 30.4 \mathrm{mmol})$ was added to the mixture, which was warmed to $40{ }^{\circ} \mathrm{C}$ and stirred for $3 \mathrm{~h}$. The reaction was quenched with sat. aq. $\mathrm{NH}_{4} \mathrm{Cl}$. The mixture was filthered through a Cerite pad, and the filtrate was $\left.\mathrm{CHCl}_{3} / \mathrm{MeOH}\right)$ to afford $\mathbf{S 2 2}(3.8 \mathrm{~g}, 28.8 \mathrm{mmol}, 95 \%)$ as a white solid. ${ }^{1} \mathrm{H} \mathrm{NMR}\left(\mathrm{CD}_{3} \mathrm{OD}, 400 \mathrm{MHz}\right) \delta 5.90(\mathrm{~m}, 1 \mathrm{H}$, H-5), $5.13\left(\mathrm{~d}, 1 \mathrm{H}, \mathrm{H}-6 \mathrm{~b}, J_{6 \mathrm{~b}, 5}=17.4 \mathrm{~Hz}\right), 5.07\left(\mathrm{~d}, 1 \mathrm{H}, \mathrm{H}-6 \mathrm{a}, J_{6 \mathrm{a}, 5}=10.1 \mathrm{~Hz}\right), 3.74\left(\mathrm{dd}, 1 \mathrm{H}, \mathrm{H}-1, J_{\mathrm{gem}}=11.5, J_{1,2}=\right.$ $3.7 \mathrm{~Hz}), 3.56$ (m, 1H, H-1), 3.55 (m, 1H, H-2), 3.46 (m, 1H, H-3), 2.45 (m, 1H, H-4), 2.19 (m, 1H, H-4); ${ }^{13} \mathrm{C} \mathrm{NMR}$ $\left(\mathrm{CD}_{3} \mathrm{OD}, 100 \mathrm{MHz}\right) \delta 136.6,117.3,75.8,73.1,64.6,38.8 ;$ ESIMS-LR $m / z 155\left[(\mathrm{M}+\mathrm{Na})^{+}\right] ;[\alpha]^{20} \mathrm{D}+7.9(c 0.90$, $\mathrm{MeOH})$. This is a known compound. ${ }^{\mathrm{S} 9}$ 


\section{(2S,3R)-1-tert-Butyldimethylsilyloxyhex-5-ene-2,3-diol (S23)}<smiles>CCCCC(C)C(C)O</smiles>

A solution of $\mathbf{S 2 2}(1.6 \mathrm{~g}, 12 \mathrm{mmol})$ in $\mathrm{CH}_{2} \mathrm{Cl}_{2}(120 \mathrm{~mL})$ was treated with imidazole $(1.22 \mathrm{~g}, 18$ mmol) and $\mathrm{TBSCl}(1.8 \mathrm{~g}, 12 \mathrm{mmol})$ at $0{ }^{\circ} \mathrm{C}$. The mixture was warmed to room temperature and stirred for $1 \mathrm{~h}$. The reaction was quenched with $\mathrm{H}_{2} \mathrm{O}$, and the mixture was partitioned between $\mathrm{CH}_{2} \mathrm{Cl}_{2}$ and $\mathrm{H}_{2} \mathrm{O}$. The organic phase was washed with brine, dried $\left(\mathrm{Na}_{2} \mathrm{SO}_{4}\right)$, filtered and concentrated in vacuo. The residue was purified by hi-flash silica gel column chromatography $(\phi 4.6 \times 12 \mathrm{~cm}, 13 \rightarrow$ $32 \% \mathrm{EtOAc/hexane)}$ to afford $\mathbf{S 2 3}(2.38 \mathrm{~g}, 9.66 \mathrm{mmol}, 80 \%)$ as a colorless oil. ${ }^{1} \mathrm{H}$ NMR $\left(\mathrm{CDCl}_{3}, 400 \mathrm{MHz}\right) \delta 5.84$ (m, 1H, H-5), 5.13 (d, 1H, H-6b, J6b,5 $=17.4$ Hz), 5.10 (d, 1H, H-6a, $J_{6 \mathrm{a}, 5}=9.6 \mathrm{~Hz}$ ), 3.73 (m, 3H, H-1, H-2), 3.52 (br s, 1H, H-3), 2.75 (br s, 1H, H-OH), 2.59 (d, 1H, H-OH, $J=4.6 \mathrm{~Hz}$ ), 2.32 (dd, 2H, H-4, $\left.J_{4,3}=J_{4,5}=6.9 \mathrm{~Hz}\right), 0.88$ (s, 9H, $\left.\mathrm{H}-{ }^{\mathrm{t}} \mathrm{Bu}\right), 0.08$ (s, 6H, H-SiCH 3$) ;{ }^{13} \mathrm{C} \mathrm{NMR}\left(\mathrm{CDCl}_{3}, 100 \mathrm{MHz}\right) \delta$ 134.7, 118.3, 73.0, 72.0, 64.1, 38.0, 26.0, 18.4, -5.3 ; ESIMS-LR $m / z 269\left[(\mathrm{M}+\mathrm{Na})^{+}\right] ;[\alpha]^{20}{ }_{\mathrm{D}}-7.3\left(c 1.10, \mathrm{CHCl}_{3}\right)$.

\section{[(4S,5R)-5-tert-Butyldimethylsilyloxymethyl-2,2-dimethyl-1,3-dioxolan-4-yl] propene (S24)}<smiles>C=CCC1OC(C)(C)O[C@@H]1COCC</smiles>

A solution of $\mathbf{S 2 3}(20 \mathrm{mg}, 0.081 \mathrm{mmol})$ in $\mathrm{CH}_{2} \mathrm{Cl}_{2}(1 \mathrm{~mL})$ was treated with dimethoxypropane $(49 \mu \mathrm{L}, 9.4 \mathrm{mmol})$ and $\mathrm{CSA}(2 \mathrm{mg}, 8.1 \mu \mathrm{mol})$ at $0{ }^{\circ} \mathrm{C}$. The mixture was warmed to room temperature and stirred for $30 \mathrm{~min}$. The reaction was quenched with sat. aq. $\mathrm{NaHCO}_{3}$. The mixture was partitioned between $\mathrm{CH}_{2} \mathrm{Cl}_{2}$ and $\mathrm{H}_{2} \mathrm{O}$. The organic phase was dried $\left(\mathrm{Na}_{2} \mathrm{SO}_{4}\right)$, filtered $\rightarrow 12 \%$ EtOAc/hexane) to afford $\mathbf{S 2 4}(22 \mathrm{mg}, 0.0768 \mathrm{mmol}, 95 \%)$ as a colorless oil. ${ }^{1} \mathrm{H} \mathrm{NMR}\left(\mathrm{CDCl}_{3}, 400 \mathrm{MHz}\right) \delta$ $5.89\left(\mathrm{ddd}, 1 \mathrm{H}, \mathrm{H}-5, J_{5,6}=17.4, J_{5,6}=10.6, J_{5,4}=6.9 \mathrm{~Hz}\right), 5.12\left(\mathrm{dd}, 1 \mathrm{H}, \mathrm{H}-6, J_{6,5}=17.4, J_{g e m}=1.8 \mathrm{~Hz}\right), 5.09(\mathrm{dd}, 1 \mathrm{H}$, $\left.\mathrm{H}-6, J_{6,5}=10.6, J_{\text {gem }}=1.8 \mathrm{~Hz}\right), 4.20\left(\mathrm{ddd}, 1 \mathrm{H}, \mathrm{H}-3, J_{3,4}=9.1, J_{3,2}=7.8, J_{3,4}=4.6 \mathrm{~Hz}\right), 4.10\left(\mathrm{dd}, 1 \mathrm{H}, \mathrm{H}-2, J_{2,3}=7.8\right.$, $\left.J_{2,1}=7.3, J_{2,1}=5.0 \mathrm{~Hz}\right), 3.69\left(\mathrm{dd}, 1 \mathrm{H}, \mathrm{H}-1, J_{1,2}=7.3, J_{\text {gem }}=10.5 \mathrm{~Hz}\right), 3.64\left(\mathrm{dd}, 1 \mathrm{H}, \mathrm{H}-1, J_{1,2}=5.0, J_{\text {gem }}=10.5 \mathrm{~Hz}\right)$, 2.37 (m, 2H, H-4), 1.43 (s, 3H, H-OC( $\left.\left(\mathrm{CH}_{3}\right)_{2} \mathrm{O}\right), 1.34$ (s, 3H, H-OC( $\left.\left.\mathrm{CH}_{3}\right)_{2} \mathrm{O}\right), 0.89$ (s, 9H, H- $\left.{ }^{-} \mathrm{Bu}\right), 0.06$ (s, 6H, H$\left.\mathrm{SiCH}_{3}\right)$; ESIMS-LR $m / z 309\left[(\mathrm{M}+\mathrm{Na})^{+}\right] ;[\alpha]^{20}{ }_{\mathrm{D}}+18.4\left(c 1.60, \mathrm{CHCl}_{3}\right)$. This is a known compound. ${ }^{\mathrm{S} 10}$

\section{(S)-4-Benzyl-3-\{(2S,3R)-4-[(4R,5S)-5-tert-butyldimethylsilyloxymethyl-2,2-dimethyl-1,3-dioxolan-4-yl]-3-}

\section{hydroxy-2-methylbutanoyl\}oxazolidin-2-one (S26)}

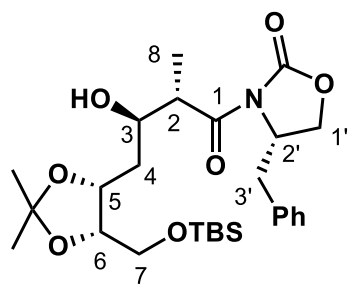

A solution of $\mathbf{S 2 4}(1.14 \mathrm{~g}, 3.98 \mathrm{mmol})$ in dioxane $(30 \mathrm{~mL})$ and $\mathrm{H}_{2} \mathrm{O}(10 \mathrm{~mL})$ was treated with 2,6-lutidine ( $922 \mu \mathrm{L}, 7.96 \mathrm{mmol}), \mathrm{K}_{2} \mathrm{OsO}_{4} \cdot 2 \mathrm{H}_{2} \mathrm{O}(2.9 \mathrm{mg}, 0.08 \mathrm{mmol})$ and $\mathrm{NaIO}_{4}(3.4 \mathrm{~g}, 15.9 \mathrm{mmol})$ at $0{ }^{\circ} \mathrm{C}$. The mixture was warmed to room temperture and stirred for $2 \mathrm{~h}$. The mixture was partitioned between $\mathrm{CH}_{2} \mathrm{Cl}_{2}$ and $\mathrm{H}_{2} \mathrm{O}$, and the organic phase was washed with $1 \mathrm{M}$ aq. $\mathrm{HCl}$, sat. aq. $\mathrm{NaHCO}_{3}, \mathrm{H}_{2} \mathrm{O}$ and brine, dried $\left(\mathrm{Na}_{2} \mathrm{SO}_{4}\right)$, filtered and concentrated in vacuo. The residue was purified by hi-flash silica gel column chromatography $(\phi 2.6 \times 10 \mathrm{~cm}, 4 \rightarrow$ 
$20 \%$ EtOAc/hexane) to afford $\mathbf{2 3}(1.19 \mathrm{~g})$ as a brown oil. A solution of $\mathbf{2 3}(1.39 \mathrm{~g}, 5.97 \mathrm{mmol})$ in $\mathrm{CH}_{2} \mathrm{Cl}_{2}(30 \mathrm{~mL})$ was treated with $\mathrm{TiCl}_{4}(654 \mathrm{mg}, 5.97 \mathrm{mmol})$ at $0{ }^{\circ} \mathrm{C}$ for $15 \mathrm{~min} . N, N$-Diisopropylethylamine $(1.04 \mathrm{~mL}, 5.97 \mathrm{mmol})$ was added to the mixture, which was stirred for $20 \mathrm{~min}$. $N$-Methylpyrrolidinone ( $594 \mu \mathrm{L}, 5.97 \mathrm{mmol}$ ) was added to the mixture, which was stirred for $10 \mathrm{~min}$. A solution of $\mathbf{S 2 5}(1.19 \mathrm{~g}, 3.98 \mathrm{mmol})$ in $\mathrm{CH}_{2} \mathrm{Cl}_{2}(10 \mathrm{~mL})$ was added to the mixture via cannula, and the whole mixture was stirred for $3 \mathrm{~h}$. The reaction was quenched with sat. aq. $\mathrm{NH}_{4} \mathrm{Cl}$. The organic phase was washed with $1 \mathrm{M}$ aq. $\mathrm{HCl}$, sat. aq. $\mathrm{NaHCO}_{3}, \mathrm{H}_{2} \mathrm{O}$ and brine, dried $\left(\mathrm{Na}_{2} \mathrm{SO}_{4}\right)$, filtered and concentrated in vacuo. The residue was purified by flash silica gel column chromatography $(\phi 4 \times 20 \mathrm{~cm}, 17 \rightarrow 20 \%$ EtOAc/hexane) to afford $\mathbf{S 2 6}(1.36 \mathrm{~g}, 2.6 \mathrm{mmol}, 65 \%$ over 2 steps $)$ as a white solid. ${ }^{1} \mathrm{H} \mathrm{NMR}\left(\mathrm{CDCl}_{3}, 400 \mathrm{MHz}\right) \delta$ 7.26 (m, 5H, H-Ph), 4.67 (ddt, 1H, H-2', $\left.J_{2^{\prime}, 1^{\prime}}=7.3, J_{2^{\prime}, 3^{\prime}}=9.6, J_{2^{\prime}, 3^{\prime}}=3.4 \mathrm{~Hz}\right), 4.43\left(\mathrm{ddd}, 1 \mathrm{H}, \mathrm{H}-5, J_{5,4}=8.2, J_{5,4}=\right.$ $5.5, J_{5,6}=8.2 \mathrm{~Hz}$ ), 4.17 (m, 4H, H-3, H-6, H-1'), 3.77 (dq, $1 \mathrm{H}, \mathrm{H}-2, J_{2,3}=4.6, J_{2,8}=7.4 \mathrm{~Hz}$ ), 3.66 (dd, $1 \mathrm{H}, \mathrm{H}-7, J_{7,6}$ $\left.=8.7, J_{g e m}=10.6 \mathrm{~Hz}\right), 3.57\left(\mathrm{dd}, 1 \mathrm{H}, \mathrm{H}-7, J_{7,6}=4.6, J_{g e m}=10.6 \mathrm{~Hz}\right), 3.41\left(\mathrm{~d}, 1 \mathrm{H}, \mathrm{H}-\mathrm{OH}, J_{\mathrm{OH}, 3}=5.3 \mathrm{~Hz}\right), 3.24(\mathrm{dd}$, $\left.1 \mathrm{H}, \mathrm{H}-3^{\prime}, J_{3^{\prime}, 2^{\prime}}=3.4, J_{\text {gem }}=13.5 \mathrm{~Hz}\right), 2.78\left(\mathrm{dd}, 1 \mathrm{H}, \mathrm{H}-3^{\prime}, J_{3^{\prime}, 2^{\prime}}=9.6, J_{\text {gem }}=13.5 \mathrm{~Hz}\right), 1.90\left(\mathrm{ddd}, 1 \mathrm{H}, \mathrm{H}-4, J_{4,3}=10.5\right.$, $\left.J_{4,5}=5.5, J_{\text {gem }}=14.2 \mathrm{~Hz}\right), 1.74\left(\mathrm{ddd}, 1 \mathrm{H}, \mathrm{H}-4, J_{4,3}=2.8, J_{4,5}=8.2, J_{\text {gem }}=14.2 \mathrm{~Hz}\right), 1.38\left(\mathrm{~s}, 3 \mathrm{H}, \mathrm{H}-\mathrm{OC}(\mathrm{CH})_{2} \mathrm{O}\right), 1.34$ (s, 3H, H-OC( $\left.\left.\mathrm{CH}_{3}\right)_{2} \mathrm{O}\right), 1.28$ (d, 3H, H-8, $\left.J_{8,2}=7.4 \mathrm{~Hz}\right), 0.90$ (s, 9H, H- $\left.{ }^{\mathrm{t}} \mathrm{Bu}\right), 0.08\left(\mathrm{~s}, 6 \mathrm{H}, \mathrm{H}-\mathrm{SiCH}_{3}\right) ;{ }^{13} \mathrm{C} \mathrm{NMR}\left(\mathrm{CDCl}_{3}\right.$, $100 \mathrm{MHz}) \delta 176.9,153.1,135.2,129.6,129.1,127.6,107.6,74.9,69.4,66.3,62.0,60.5,55.3,43.4,37.9,33.4,28.3$, 26.0, 25.6, 21.2, 18.4 14.3, 11.5, 0.13, -5.3; ESIMS-LR m/z 544 [(M+Na) $)^{+}$; HRMS (ESI) m/z: [(M+Na $\left.)^{+}\right]$Calcd for $\mathrm{C}_{27} \mathrm{H}_{43} \mathrm{NO}_{7} \mathrm{NaSi} 544.2706$; Found 544.2726; $[\alpha]^{20}{ }_{\mathrm{D}}+35.4\left(c 1.00, \mathrm{CHCl}_{3}\right)$.

\section{(S)-4-Benzyl-3-\{(2S,3R)-4-[(4R,5S)-5-tert-butyldimethylsilyloxymethyl-2,2-dimethyl-1,3-dioxolan-4-yl]-3-}

\section{methoxymethoxy-2-methylbutanoyl\}oxazolidin-2-one (S27)}

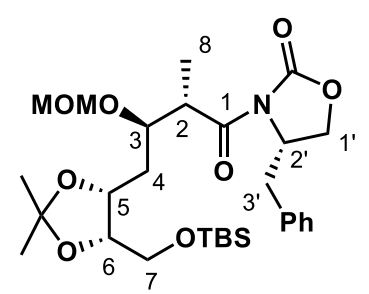

A solution of $\mathbf{S 2 6}(1.36 \mathrm{~g}, 2.6 \mathrm{mmol})$ in $\mathrm{CH}_{2} \mathrm{Cl}_{2}(26 \mathrm{~mL})$ was treated with ${ }^{i} \mathrm{Pr}_{2} \mathrm{NEt}(3.62$ $\mathrm{mL}, 20.8 \mathrm{mmol})$, TBAI $(96 \mathrm{mg}, 0.26 \mathrm{mmol})$ and $\mathrm{MOMCl}(790 \mu \mathrm{L}, 10.4 \mathrm{mmol})$ at room temperature for $48 \mathrm{~h}$. The reaction was quenched with $\mathrm{H}_{2} \mathrm{O}$. The mixture was partitioned between EtOAc and $\mathrm{H}_{2} \mathrm{O}$. The organic phase was washed with $1 \mathrm{M}$ aq. $\mathrm{HCl}$, sat. aq. $\mathrm{NaHCO}_{3}, \mathrm{H}_{2} \mathrm{O}$ and brine, dried $\left(\mathrm{Na}_{2} \mathrm{SO}_{4}\right)$, filtered and concentrated in vacuo. The residue was purified by hi-flash silica gel column chromatography $(\phi 2.6 \times 10 \mathrm{~cm}, 20 \rightarrow 30 \%$ EtOAc/hexane $)$ to afford $\mathbf{S 2 7}$ (1.37 g, $2.42 \mathrm{mmol}, 93 \%)$ as a colorless oil. ${ }^{1} \mathrm{H} \mathrm{NMR}\left(\mathrm{CDCl}_{3}, 400 \mathrm{MHz}\right) \delta 7.25$ (m, 5H, H-Ph), 4.67 (s, 2H, H$\left.\mathrm{OCH}_{2} \mathrm{O}\right), 4.60\left(\mathrm{~m}, 1 \mathrm{H}, \mathrm{H}-2^{\prime}\right), 4.30\left(\mathrm{ddd}, 1 \mathrm{H}, \mathrm{H}-5, J_{5,4}=11.0, J_{5,4}=2.3, J_{5,6}=5.9 \mathrm{~Hz}\right), 4.16\left(\mathrm{~m}, 3 \mathrm{H}, \mathrm{H}-3, \mathrm{H}-1^{\prime}\right), 4.06$

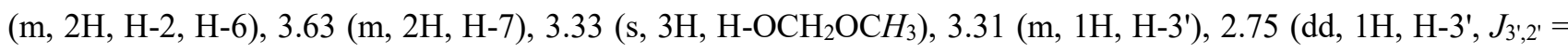
$\left.10.5, J_{g e m}=12.8 \mathrm{~Hz}\right), 1.89(\mathrm{~m}, 1 \mathrm{H}, \mathrm{H}-4), 1.75(\mathrm{~m}, 1 \mathrm{H}, \mathrm{H}-4), 1.41\left(\mathrm{~s}, 3 \mathrm{H}, \mathrm{H}-\mathrm{OC}\left(\mathrm{CH}_{3}\right)_{2} \mathrm{O}\right), 1.32\left(\mathrm{~s}, 3 \mathrm{H}, \mathrm{H}-\mathrm{OC}(\mathrm{CH})_{2} \mathrm{O}\right)$, $1.24\left(\mathrm{~d}, 3 \mathrm{H}, \mathrm{H}-8, J_{8,2}=5.9 \mathrm{~Hz}\right), 0.88\left(\mathrm{~s}, 9 \mathrm{H}, \mathrm{H}-{ }^{t} \mathrm{Bu}\right), 0.06\left(\mathrm{~s}, 6 \mathrm{H}, \mathrm{H}-\mathrm{SiCH}_{3}\right) ;{ }^{13} \mathrm{C} \mathrm{NMR}\left(\mathrm{CDCl}_{3}, 100 \mathrm{MHz}\right) \delta 174.8$, 153.4, 135.7, 129.6, 129.1, 127.4, 108.1, 97.3, 73.7, 66.2, 62.0, 56.4, 56.2, 42.1, 37.9, 32.5, 28.4, 26.0, 25.7, 18.3, 11.7, 6.6, -5.3; ESIMS-LR $m / z 588\left[(\mathrm{M}+\mathrm{Na})^{+}\right]$; HRMS (ESI) m/z: $\left[(\mathrm{M}+\mathrm{Na})^{+}\right]$Calcd for $\mathrm{C}_{29} \mathrm{H}_{47} \mathrm{NO}_{8} \mathrm{NaSi} 588.2969$; Found 588.2966; $[\alpha]^{20} \mathrm{D}+52.6\left(c 0.95, \mathrm{CHCl}_{3}\right)$. 
$(R, Z)-3-M e t h y l o c t-2-e n-6-y n-4-y l ~(2 S, 3 R)-4-[(4 R, 5 S)-5-h y d r o x y m e t h y l-2,2-d i m e t h y l-1,3-d i o x o l a n-4-y l]-3-$ methoxymethoxy-2-methylbutanoate (42)

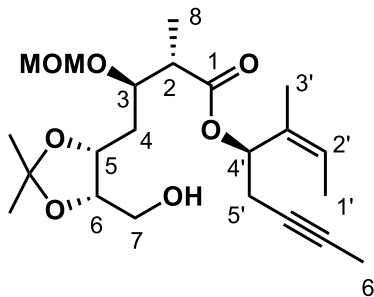

A solution of $\mathbf{S 2 7}(283 \mathrm{mg}, 0.5 \mathrm{mmol})$ in THF (4 mL) and $\mathrm{H}_{2} \mathrm{O}(1 \mathrm{~mL})$ was treated with $\mathrm{H}_{2} \mathrm{O}_{2}\left(228 \mu \mathrm{L}, 2 \mathrm{mmol}, 30 \%\right.$ in $\left.\mathrm{H}_{2} \mathrm{O}\right)$ and $\mathrm{LiOH}(42 \mathrm{mg}, 1.0 \mathrm{mmol})$ at $0{ }^{\circ} \mathrm{C}$ for $3 \mathrm{~h}$. The reaction was quenched with sat. aq. $\mathrm{Na}_{2} \mathrm{~S}_{2} \mathrm{O}_{3}$. The mixture was partitioned between $\mathrm{Et}_{2} \mathrm{O}$ and sat. aq. $\mathrm{NaHCO}_{3}$. The organic phase was washed with brine, dried $\left(\mathrm{Na}_{2} \mathrm{SO}_{4}\right)$,

filtered and concentrated in vacuo to give S28. A solution of the residue and $\mathbf{1 0}(59 \mathrm{mg}$, $0.428 \mathrm{mmol})$ in $\mathrm{CH}_{2} \mathrm{Cl}_{2}(3 \mathrm{~mL})$ was treated with DMAP (4 mg, $\left.28.5 \mu \mathrm{mol}\right)$ and EDCI (109 mg, $\left.0.57 \mathrm{mmol}\right)$ at room temperature for $13 \mathrm{~h}$. The mixture was partitioned between EtOAc and $\mathrm{H}_{2} \mathrm{O}$. The organic phase was washed with 1 $\mathrm{M}$ aq. $\mathrm{HCl}$, sat. aq. $\mathrm{NaHCO}_{3}, \mathrm{H}_{2} \mathrm{O}$ and brine, dried $\left(\mathrm{Na}_{2} \mathrm{SO}_{4}\right)$, filtered and concentrated in vacuo to give S29. A solution of the residue in THF $(3 \mathrm{~mL})$ was treated with $3 \mathrm{HF} \cdot \mathrm{Et}_{3} \mathrm{~N}(465 \mu \mathrm{L}, 2.85 \mathrm{mmol})$ at room temperature for 19 h. The mixture was partitioned between EtOAc and $\mathrm{H}_{2} \mathrm{O}$. The organic phase was washed with $1 \mathrm{M}$ aq. $\mathrm{HCl}$, sat. aq. $\mathrm{NaHCO}_{3}, \mathrm{H}_{2} \mathrm{O}$ and brine, dried $\left(\mathrm{Na}_{2} \mathrm{SO}_{4}\right)$, filtered and concentrated in vacuo. The residue was purified by hi-flash silica gel column chromatography $(\phi 2.0 \times 6.5 \mathrm{~cm}, 20 \rightarrow 35 \rightarrow 50 \%$ EtOAc/hexane $)$ to afford $42(42 \mathrm{mg}, 0.10 \mathrm{mmol}$, $36 \%$ over 3 steps $)$ as a colorless oil. ${ }^{1} \mathrm{H}$ NMR $\left(\mathrm{CDCl}_{3}, 400 \mathrm{MHz}\right) \delta 5.72\left(\mathrm{dd}, 1 \mathrm{H}, \mathrm{H}-4^{\prime}, J_{4^{\prime}, 5^{\prime}}=J_{4^{\prime}, 5^{\prime}}=5.0 \mathrm{~Hz}\right), 5.44(\mathrm{q}$, $\left.1 \mathrm{H}, \mathrm{H}-2^{\prime}, J_{2^{\prime}, 1^{\prime}}=6.8 \mathrm{~Hz}\right), 4.71\left(\mathrm{~d}, 1 \mathrm{H}, \mathrm{H}-9, J_{\text {gem }}=6.9 \mathrm{~Hz}\right), 4.65\left(\mathrm{~d}, 1 \mathrm{H}, \mathrm{H}-9, J_{\text {gem }}=6.9 \mathrm{~Hz}\right), 4.33\left(\mathrm{ddd}, 1 \mathrm{H}, \mathrm{H}-5, J_{5,4}\right.$ $\left.=9.6, J_{5,6}=6.4, J_{5,4}=3.6 \mathrm{~Hz}\right), 4.15\left(\mathrm{dt}, 1 \mathrm{H}, \mathrm{H}-6, J_{6,5}=6.4, J_{6,7}=4.6 \mathrm{~Hz}\right), 4.01\left(\mathrm{ddd}, 1 \mathrm{H}, \mathrm{H}-3, J_{3,4}=7.8, J_{3,2}=4.6\right.$, $\left.J_{3,4}=3.6 \mathrm{~Hz}\right), 3.60(\mathrm{~m}, 2 \mathrm{H}, \mathrm{H}-7), 3.37\left(\mathrm{~s}, 3 \mathrm{H}, \mathrm{OCH}_{2} \mathrm{OCH}_{3}\right), 2.76\left(\mathrm{dq}, 1 \mathrm{H}, \mathrm{H}-2, J_{2,3}=4.6, J_{2,8}=7.3 \mathrm{~Hz}\right), 2.75(\mathrm{ddq}$, $\left.1 \mathrm{H}, \mathrm{H}-5^{\prime}, J_{\text {gem }}=16.5, J_{5^{\prime}, 4^{\prime}}=5.0, J_{5^{\prime}, 6^{\prime}}=2.8 \mathrm{~Hz}\right), 2.41\left(\mathrm{ddq}, 1 \mathrm{H}, \mathrm{H}-5^{\prime}, J_{g e m}=16.5, J_{5^{\prime}, 4^{\prime}}=5.0, J_{5^{\prime}, 6^{\prime}}=2.3 \mathrm{~Hz}\right), 1.96(\mathrm{br}$ s, 1H, H-OH), 1.81 (m, 1H, H-4), 1.75 (m, 3H, H-1'), 1.72 (m, 3H, H-6'), 1.71 (m, 1H, H-4), 1.66 (m, 3H, H-3'), 1.45 $\left(\mathrm{s}, 3 \mathrm{H}, \mathrm{H}-\mathrm{OC}\left(\mathrm{CH}_{3}\right)_{2} \mathrm{O}\right), 1.35$ (s, 3H, H-OC $\left.\left(\mathrm{CH}_{3}\right)_{2} \mathrm{O}\right), 1.16\left(\mathrm{~d}, 3 \mathrm{H}, \mathrm{H}-8, J_{8,2}=7.3 \mathrm{~Hz}\right) ;{ }^{13} \mathrm{C} \mathrm{NMR}\left(\mathrm{CDCl}_{3}, 100 \mathrm{MHz}\right)$ $\delta 173.3,132.4,124.8,108.2,97.2,75.6,71.0,61.2,56.1,44.0,31.7,28.3,25.6,23.0,18.0,13.4,12.2$, 3.6 ; ESIMSLR $m / z 435\left[(\mathrm{M}+\mathrm{Na})^{+}\right]$; HRMS (ESI) m/z: $\left[(\mathrm{M}+\mathrm{Na})^{+}\right]$Calcd for $\mathrm{C}_{22} \mathrm{H}_{36} \mathrm{O}_{7} \mathrm{Na}$ 435.2359; Found 435.2333; $[\alpha]^{20}{ }_{\mathrm{D}}$ $+14.2\left(c 1.10, \mathrm{CHCl}_{3}\right)$.

$(R, Z)-3-M e t h y l o c t-2-e n-6-y n-4-y l ~(2 S, 3 R)-4-[(4 R, 5 R)-5-f o r m y l-2,2-d i m e t h y l-1,3-d i o x o l a n-4-y l]-3-$ methoxymethoxy-2-methylbutanoate (S30)

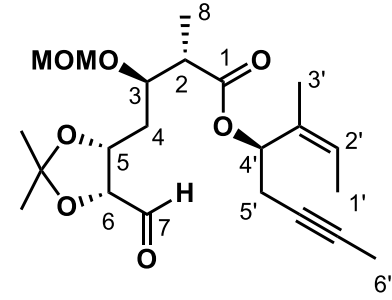

A solution of $42(11 \mathrm{mg}, 26 \mu \mathrm{mol})$ in $\mathrm{CH}_{2} \mathrm{Cl}_{2}(1 \mathrm{~mL})$ was treated with Dess-Martin periodinane $(23 \mathrm{mg}, 50 \mu \mathrm{mol})$ at room temperature for $30 \mathrm{~min}$. The reaction was quenched with sat. aq. $\mathrm{Na}_{2} \mathrm{~S}_{2} \mathrm{O}_{3}$ and sat. aq. $\mathrm{NaHCO}_{3}$. The mixture was partitioned between EtOAc and $\mathrm{H}_{2} \mathrm{O}$. The organic phase was washed with brine, dried $\left(\mathrm{Na}_{2} \mathrm{SO}_{4}\right)$, filtered and concentrated in vacuo. The residue was purified by silica gel column 
chromatography ( $\phi 2.0 \times 6.5 \mathrm{~cm}, 50 \%$ EtOAc/hexane) to afford a crude $\mathbf{S 3 0}(11 \mathrm{mg})$ as a colorless oil. The crude $\mathbf{S 3 0}$ was used for the next reaction without further purification. ${ }^{1} \mathrm{H} \mathrm{NMR}\left(\mathrm{CDCl}_{3}, 400 \mathrm{MHz}\right) \delta 9.64(\mathrm{~s}, 1 \mathrm{H}, \mathrm{CHO}), 5.71$ (m, 1H, H-4'), 5.44 (m, 1H, H-2'), 4.69 (m, 1H, H-OCH $2 \mathrm{O}), 4.65$ (m, 1H, H-9), 4.55 (m, 1H, H-6), 4.28 (m, 1H, H5), 4.02 (m, 1H, H-3), 3.36 (s, 3H, H-OCH $\mathrm{OCH}_{3}$ ), 2.70 (m, 1H, H-2), 2.52 (m, 1H, H-5'), 2.41 (m, 1H, H-5'), 1.92 (m, 1H, H-4), 1.74 (m, 3H, H-1'), 1.65 (m, 4H, H-4, H-6'), 1.70 (m, 3H, H-3'), 1.40 (s, 6H, H-OC(CH3) 2 ), 1.14 (m, $3 \mathrm{H}, \mathrm{H}-8)$.

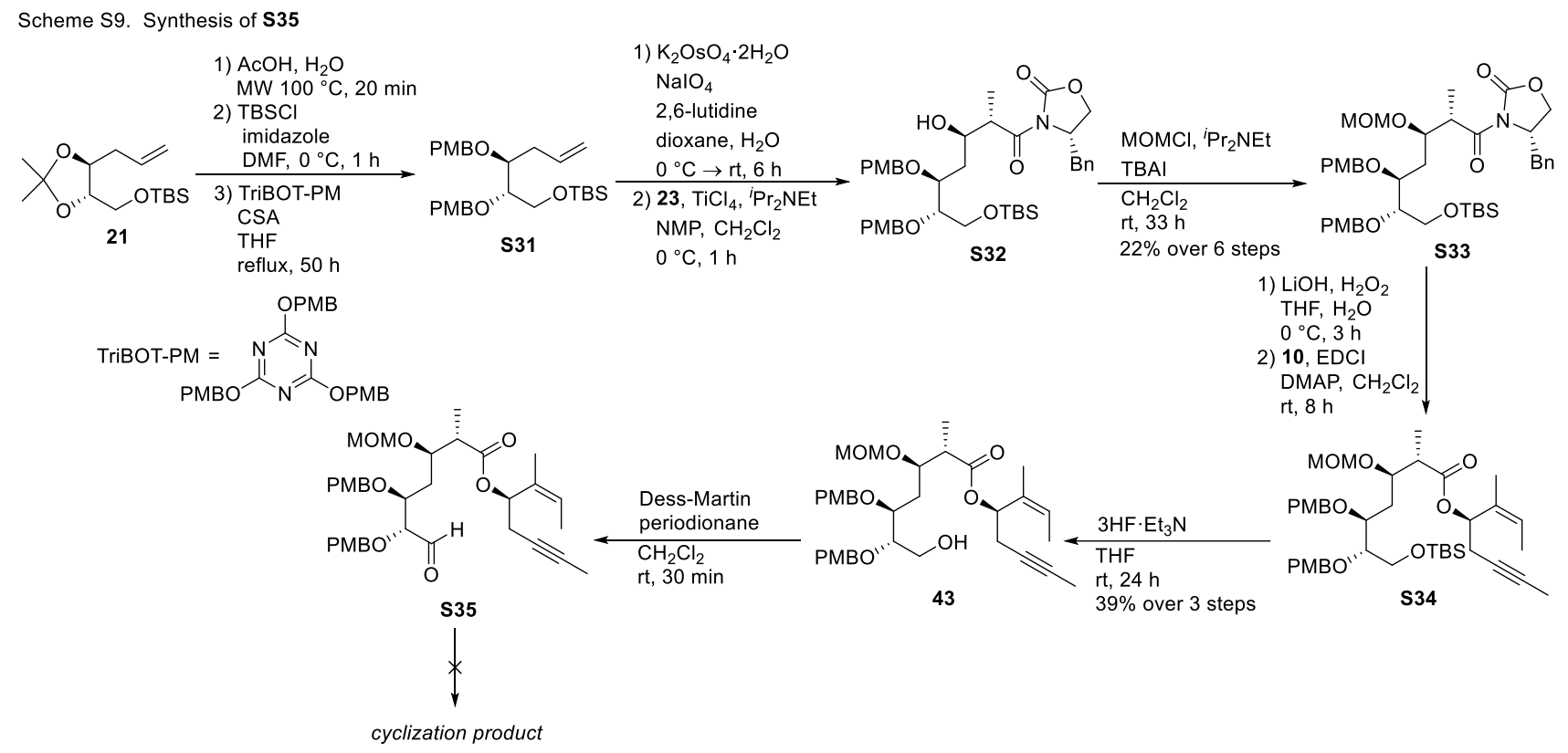

(S)-4-Benzyl-3[(2S,3R,5S,6S)-5,6-7-tert-butyldimethylsilyloxy-bis(4-methoxybenzyloxy)-3-methoxymethoxy-

\section{2-methylheptanoyl]oxazolidin-2-one (S33)}

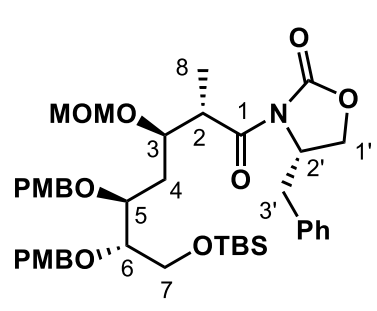

A solution of $21(859 \mathrm{mg}, 3.0 \mathrm{mmol})$ in $\mathrm{AcOH}(2 \mathrm{~mL})$ and $\mathrm{H}_{2} \mathrm{O}(2 \mathrm{~mL})$ was heated at $100{ }^{\circ} \mathrm{C}$ under microwave irradiation for $20 \min (0 \mathrm{bar}, 10 \mathrm{~W})$. The mixture was concentrated in vacuo. A solution of the residue in $\mathrm{CH}_{2} \mathrm{Cl}_{2}(30 \mathrm{~mL})$ was treated with imidazole (306 mg, $4.5 \mathrm{mmol}$ ) and TBSCl ( $452 \mathrm{mg}, 3 \mathrm{mmol})$ at $0{ }^{\circ} \mathrm{C}$ for $1 \mathrm{~h}$. The reaction was quenched with $\mathrm{MeOH}$. The mixture was extracted with $\mathrm{CH}_{2} \mathrm{Cl}_{2}$. The organic phase was dried $\left(\mathrm{Na}_{2} \mathrm{SO}_{4}\right)$, filtered and concentrated in vacuo. The residue was semi-purified by hi-flash silica gel column chromatography $(\phi 2.6 \times 10 \mathrm{~cm}, 12 \rightarrow 32 \%$ EtOAc/hexane) to afford a crude diol. A solution of the crude diol in THF $(30 \mathrm{~mL})$ was treated with CSA $(70 \mathrm{mg}, 0.30 \mathrm{mmol})$ and TriBOT-PM $(2.9 \mathrm{~g}, 6.0 \mathrm{mmol})$ at room temperature. The mixture was warmed to $60{ }^{\circ} \mathrm{C}$ and stirred for $25 \mathrm{~h}$. 10-Camphorsulfonic acid (70 mg, $0.30 \mathrm{mmol}$ ) and TriBOT-PM (1.45 g, $6.0 \mathrm{mmol})$ were added to the mixture, which was stirred for $25 \mathrm{~h}$. The mixture was partitioned between EtOAc and $\mathrm{H}_{2} \mathrm{O}$. The organic phase was washed with $1 \mathrm{M}$ aq. $\mathrm{HCl}$, sat. aq. $\mathrm{NaHCO}_{3}, \mathrm{H}_{2} \mathrm{O}$ and brine, dried $\left(\mathrm{Na}_{2} \mathrm{SO}_{4}\right)$, filtered and concentrated in vacuo. The residue was purified by hi-flash silica gel column chromatography $(\phi 2.6 \times$ 
$10 \mathrm{~cm}, 0 \rightarrow 5 \%$ EtOAc/hexane) to afford a crude $\mathbf{S 3 1}$. A solution of the crude $\mathbf{S 3 1}$ (570 $\mathrm{mg}, 1.17 \mathrm{mmol}$ ) in dioxane $(15 \mathrm{~mL})$ and $\mathrm{H}_{2} \mathrm{O}(5 \mathrm{~mL})$ was treated with 2,6-lutidine (271 $\left.\mu \mathrm{L}, 2.34 \mathrm{mmol}\right), \mathrm{K}_{2} \mathrm{OsO}_{4} \cdot 2 \mathrm{H}_{2} \mathrm{O}(8.5 \mathrm{mg}, 23 \mu \mathrm{mol})$ and $\mathrm{NaIO}_{4}(1.0 \mathrm{~g}, 4.68 \mathrm{mmol})$ at $0{ }^{\circ} \mathrm{C}$. The mixture was warmed to room temperture and stirred for $6 \mathrm{~h}$. The mixture was partitioned between $\mathrm{CH}_{2} \mathrm{Cl}_{2}$ and $\mathrm{H}_{2} \mathrm{O}$. The organic phase was washed with $1 \mathrm{M}$ aq. $\mathrm{HCl}$, sat. aq. $\mathrm{NaHCO}_{3}, \mathrm{H}_{2} \mathrm{O}$ and brine, dried $\left(\mathrm{Na}_{2} \mathrm{SO}_{4}\right)$, filtered and concentrated in vacuo. The residue was purified by hi-flash silica gel column chromatography $(\phi 2.6 \times 10 \mathrm{~cm}, 5 \rightarrow 20 \%$ EtOAc/hexane) to afford a crude aldehyde. A solution of $\mathbf{2 3}$ (268 mg, 1.15 $\mathrm{mmol})$ in $\mathrm{CH}_{2} \mathrm{Cl}_{2}$ was treated with $\mathrm{TiCl}_{4}(126 \mu \mathrm{L}, 1.15 \mathrm{mmol})$ at $0{ }^{\circ} \mathrm{C}$ for $15 \mathrm{~min} . N, N$-Diisopropylethylamine (200 $\mu \mathrm{L}, 1.15 \mathrm{mmol})$ was added to the mixture, which was stirred for $20 \mathrm{~min} . N$-Methylpyrrolidinone $(109 \mu \mathrm{L}, 1.15 \mathrm{mmol})$ was added to the mixture, which was stirred for $10 \mathrm{~min}$. A solution of the crude aldehyde obtained above ( $380 \mathrm{mg}$ ) in $\mathrm{CH}_{2} \mathrm{Cl}_{2}(2 \mathrm{~mL})$ was added to the mixture via cannula and the whole mixture was stirred for $1 \mathrm{~h}$. The reaction was quenched with sat. aq. $\mathrm{NH}_{4} \mathrm{Cl}$. The organic phase was washed with $1 \mathrm{M} a q . \mathrm{HCl}$, sat. aq. $\mathrm{NaHCO}_{3}, \mathrm{H}_{2} \mathrm{O}$ and brine, dried $\left(\mathrm{Na}_{2} \mathrm{SO}_{4}\right)$, filtered and concentrated in vacuo. The residue was purified by hi-flash silica gel column chromatography $(\phi 2.6 \times 10 \mathrm{~cm}, 20 \%$ EtOAc/hexane) to afford a crude $\mathbf{S 3 2}$. A solution of the crude $\mathbf{S 3 2}(630 \mathrm{mg})$ in $\mathrm{CH}_{2} \mathrm{Cl}_{2}(7 \mathrm{~mL})$ was treated with ${ }^{i} \mathrm{Pr}_{2} \mathrm{NEt}(1.07 \mathrm{~mL}, 6.16 \mathrm{mmol})$, TBAI (28 mg, $\left.77 \mu \mathrm{mol}\right)$ and MOMCl $(234 \mu \mathrm{L}, 3.08$ mmol) at room temperature for $33 \mathrm{~h}$. The reaction was quenched with $\mathrm{H}_{2} \mathrm{O}$. The mixture was partitioned between EtOAc and $\mathrm{H}_{2} \mathrm{O}$. The organic phase was washed with $1 \mathrm{M}$ aq. $\mathrm{HCl}$, sat. aq. $\mathrm{NaHCO}_{3}, \mathrm{H}_{2} \mathrm{O}$ and brine, dried $\left(\mathrm{Na}_{2} \mathrm{SO}_{4}\right)$, filtered and concentrated in vacuo. The residue was purified by hi-flash silica gel column chromatography $(\phi 2.0 \times$ $10 \mathrm{~cm}, 10 \rightarrow 30 \%$ EtOAc/hexane) to afford $\mathbf{S 3 3}$ ( $515 \mathrm{mg}, 0.67 \mathrm{mmol}, 22 \%$ over 6 steps) as a colorless oil. ${ }^{1} \mathrm{H}$ NMR $\left(\mathrm{CDCl}_{3}, 400 \mathrm{MHz}\right) \delta 7.30\left(\mathrm{~m}, 9 \mathrm{H}, \mathrm{H}-\mathrm{C}_{6} H_{4} \mathrm{OCH}_{3}, \mathrm{H}-\mathrm{Ph}\right), 6.85$ (d, 2H, H-C $\left.\mathrm{H}_{4} \mathrm{OCH}_{3}, J=8.7 \mathrm{~Hz}\right), 6.81(\mathrm{~d}, 2 \mathrm{H}, \mathrm{H}-$ $\left.\mathrm{C}_{6} \mathrm{H}_{4} \mathrm{OCH}_{3}, J=8.7 \mathrm{~Hz}\right), 4.66\left(\mathrm{~m}, 4 \mathrm{H}, \mathrm{H}-\mathrm{OCH}_{2} \mathrm{C}_{6} \mathrm{H}_{4} \mathrm{OCH}_{3}\right), 4.56\left(\mathrm{~m}, 1 \mathrm{H}, \mathrm{H}-2^{\prime}\right), 4.47$ (m, 2H, H-OCH $\left.\mathrm{O}\right), 4.18(\mathrm{~m}$, 1H, H-3), 4.00 (m, 1H, H-1'), 3.87 (m, 3H, H-5, H-6, H-1'), 3.78 (s, 3H, H-C $\left.\mathrm{C}_{6} \mathrm{H}_{4} \mathrm{OCH} \mathrm{H}_{3}\right) 3.76$ (s, 3H, H-C $6 \mathrm{H}_{4} \mathrm{OCH} \mathrm{H}_{3}$ ), 3.65 (m, 2H, H-7), 3.30 (s, 3H, H-OCH ${ }_{2} \mathrm{OCH}_{3}$ ), 3.21 (d, 1H, H-3'), 2.95 (m, 1H, H-2), 2.73 (m, 1H, H-3'), 2.03 (ddd, $\left.1 \mathrm{H}, \mathrm{H}-4, J_{g e m}=15.0, J_{4,5}=J_{4,3}=5.0 \mathrm{~Hz}\right), 1.75\left(\mathrm{ddd}, 1 \mathrm{H}, \mathrm{H}-4, J_{g e m}=15.0, J_{4,5}=7.8, J_{4,3}=5.5 \mathrm{~Hz}\right), 1.27(\mathrm{~d}, 3 \mathrm{H}, \mathrm{H}-8$,

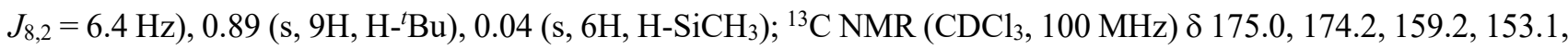
135.6, 131.1, 129.6, 129.3, 129.1, 129.0, 113.8, 96.2, 79.4, 74.5, 72.8, 71.0, 66.4, 66.0, 63.4, 56.2, 55.4, 55.3, 41.9, 38.1, 37.8, 32.4, 29.4, 26.1, 18.4, 13.3, 8.4, -5.2; ESIMS-LR $m / z 788\left[(\mathrm{M}+\mathrm{Na})^{+}\right]$; HRMS (ESI) m/z: [(M+Na) $\left.{ }^{+}\right]$ Calcd for $\mathrm{C}_{42} \mathrm{H}_{59} \mathrm{NO}_{10} \mathrm{NaSi} 788.3806$; Found 788.3817; $[\alpha]^{20}{ }_{\mathrm{D}}+41.9\left(c 0.20, \mathrm{CHCl}_{3}\right)$.

\section{$(R, Z)$-3-Methyloct-2-en-6-yn-4-yl (2S,3R,5S,6S)-7-hydroxy-5,6-bis(4-methoxybenzyloxy)-3-methoxymethoxy-}

\section{2-methylheptanoate (43)}

A solution of $\mathbf{S 3 3}(493 \mathrm{mg}, 0.64 \mathrm{mmol})$ in THF $(5.6 \mathrm{~mL})$ and $\mathrm{H}_{2} \mathrm{O}(1.4 \mathrm{~mL})$ was treated with $\mathrm{H}_{2} \mathrm{O}_{2}(291 \mu \mathrm{L}, 2.56$ 


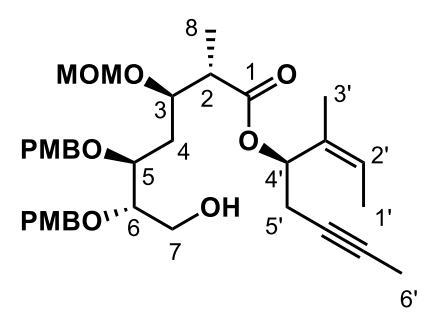

$0.79 \mathrm{mmol}$ ) at room temperature for $8 \mathrm{~h}$. The mixture was partitioned between EtOAc and $\mathrm{H}_{2} \mathrm{O}$. The organic phase was washed with $1 \mathrm{M}$ aq. $\mathrm{HCl}$, sat. aq. $\mathrm{NaHCO}_{3}, \mathrm{H}_{2} \mathrm{O}$ and brine, dried $\left(\mathrm{Na}_{2} \mathrm{SO}_{4}\right)$, filtered and concentrated in vacuo A solution of the residue $(280 \mathrm{mg})$ in THF $(4 \mathrm{~mL})$ was treated with $3 \mathrm{HF} \cdot \mathrm{Et}_{3} \mathrm{~N}(628 \mu \mathrm{L}, 3.9 \mathrm{mmol})$ at room temperature for $24 \mathrm{~h}$. The mixture was partitioned between EtOAc and $\mathrm{H}_{2} \mathrm{O}$. The organic phase was washed with $1 \mathrm{M} \mathrm{aq}$. $\mathrm{HCl}$, sat. aq. $\mathrm{NaHCO}_{3}, \mathrm{H}_{2} \mathrm{O}$ and brine, dried $\left(\mathrm{Na}_{2} \mathrm{SO}_{4}\right)$, filtered and concentrated in vacuo. The residue was purified by hiflash silica gel column chromatography $(\phi 2 \times 6.5 \mathrm{~cm}, 50 \%$ EtOAc/hexane) to afford 43 (160 mg, $0.25 \mathrm{mmol}, 39 \%$ over 3 steps) as a colorless oil. ${ }^{1} \mathrm{H}$ NMR $\left(\mathrm{CDCl}_{3}, 400 \mathrm{MHz}\right) \delta 7.26\left(\mathrm{~m}, 4 \mathrm{H}, \mathrm{H}-\mathrm{C}_{6} \mathrm{H}_{4} \mathrm{OCH}_{3}\right), 6.87(\mathrm{~m}, 4 \mathrm{H}, \mathrm{H}-$ $\left.\mathrm{C}_{6} H_{4} \mathrm{OCH}_{3}\right), 5.71\left(\mathrm{t}, 1 \mathrm{H}, \mathrm{H}-4^{\prime}, J_{4^{\prime}, 5^{\prime}}=7.3 \mathrm{~Hz}\right), 5.43\left(\mathrm{q}, 1 \mathrm{H}, \mathrm{H}-2^{\prime}, J_{2^{\prime}, 1^{\prime}}=6.4 \mathrm{~Hz}\right), 4.61\left(\mathrm{~d}, 1 \mathrm{H}, \mathrm{H}-\mathrm{OCH} \mathrm{H}_{2} \mathrm{O}, J_{g e m}=6.9\right.$ $\mathrm{Hz}), 4.60\left(\mathrm{~d}, 1 \mathrm{H}, \mathrm{H}-\mathrm{OCH}_{2} \mathrm{O}, J_{\text {gem }}=6.9 \mathrm{~Hz}\right), 4.53\left(\mathrm{~m}, 2 \mathrm{H}, \mathrm{H}-\mathrm{OCH}_{2} \mathrm{C}_{6} \mathrm{H}_{4} \mathrm{OCH}_{3}\right), 4.30\left(\mathrm{~m}, 2 \mathrm{H}, \mathrm{H}-\mathrm{OCH}_{2} \mathrm{C}_{6} \mathrm{H}_{4} \mathrm{OCH}_{3}\right)$, 4.06 (dd, 1H, H-3, $J_{3,4}=6.8 \mathrm{~Hz}, J_{3,2}=4.1$ ), 3.80 ( $, 6 \mathrm{H}, \mathrm{H}_{-} \mathrm{C}_{6} \mathrm{H}_{4} \mathrm{OCH} \mathrm{H}_{3}$ ), 3.77 (m, 2H, H-5, H-6), 3.64 (m, 2H, H-7), 3.30 (s, 3H, H-OCH ${ }_{2} \mathrm{OCH}_{3}$ ), 2.58 (dq, $1 \mathrm{H}, \mathrm{H}-2, J_{2,8}=7.3, J_{2,3}=4.1 \mathrm{~Hz}$ ), 2.50 (m, 1H, H-5'), 2.39 (m, 1H, H-5'), 1.90 (m, 3H, H-4, H-OH), 1.70 (m, 9H, H-1', H-3', H-6'), 1.12 (d, 3H, H-8, $J_{8,2}=7.3 \mathrm{~Hz}$ ); ${ }^{13} \mathrm{C}$ NMR $\left(\mathrm{CDCl}_{3}, 100 \mathrm{MHz}\right) \delta$ $173.5,159.5,132.4,130.4,129.8,124.7,114.0,96.8,79.0,74.5,72.5,72.0,71.0,61.8,55.9,55.4,43.1,32.8,22.9$, 17.9, 13.4, 11.0, 3.6; ESIMS-LR $m / z 635$ [(M+Na) $\left.)^{+}\right]$; HRMS (ESI) m/z: [(M+Na) $\left.{ }^{+}\right]$Calcd for $\mathrm{C}_{35} \mathrm{H}_{48} \mathrm{O}_{9} \mathrm{Na} 635.3196$; Found 635.3197; $[\alpha]^{20}{ }_{\mathrm{D}}+20.3\left(c 0.78, \mathrm{CHCl}_{3}\right)$.

\section{( $R, Z)-3$-Methyloct-2-en-6-yn-4-yl $(2 S, 3 R, 5 S, 6 R)$-5,6-bis(4-methoxybenzyloxy)-3-methoxymethoxy-2-methyl-}

\section{7-oxoheptanoate (S35)}

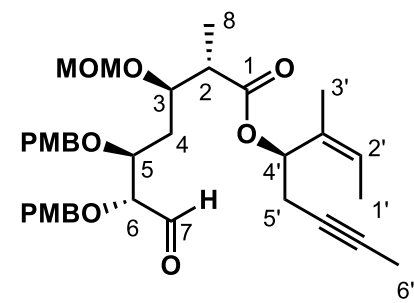

A solution of $43(10 \mathrm{mg}, 16 \mu \mathrm{mol})$ in $\mathrm{CH}_{2} \mathrm{Cl}_{2}(0.5 \mathrm{~mL})$ was treated with DessMartin periodinane $(10 \mathrm{mg}, 24 \mu \mathrm{mol})$ at room temperature for $30 \mathrm{~min}$. The mixture was diluted with hexane, filtered through a Cerite pad, and the filtrate was concentrated in vacuo. The residue was purified by silica gel column chromatography $(\phi 1 \times 8 \mathrm{~cm}$, $25 \%$ EtOAc/hexane) to afford a crude $\mathbf{S 3 5}(10 \mathrm{mg}$ ) as a colorless oil. The crude $\mathbf{S 3 5}$ was used for the next reaction without further purification. ${ }^{1} \mathrm{H} \mathrm{NMR}\left(\mathrm{CDCl}_{3}, 400 \mathrm{MHz}\right) \delta 9.66(\mathrm{~s}, 1 \mathrm{H}, \mathrm{CHO}), 7.27$ $\left(\mathrm{m}, 2 \mathrm{H}, \mathrm{H}-\mathrm{C}_{6} H_{4} \mathrm{OCH}_{3}\right), 7.18\left(\mathrm{~m}, 2 \mathrm{H}, \mathrm{H}-\mathrm{C}_{6} H_{4} \mathrm{OCH}_{3}\right), 6.85\left(\mathrm{~m}, 4 \mathrm{H}, \mathrm{H}-\mathrm{C}_{6} H_{4} \mathrm{OCH}_{3}\right), 5.72\left(\mathrm{dd}, 1 \mathrm{H}, \mathrm{H}-4^{\prime}, J_{4^{\prime}, 5^{\prime}}=J_{4^{\prime}, 5^{\prime}}=\right.$ $7.3 \mathrm{~Hz}$ ), 5.46 (q, $\left.1 \mathrm{H}, \mathrm{H}-2^{\prime}, J_{2^{\prime}, 1^{\prime}}=6.8 \mathrm{~Hz}\right), 4.48-4.60\left(\mathrm{~m}, 6 \mathrm{H}, \mathrm{H}-\mathrm{OCH}_{2} \mathrm{O}, \mathrm{H}-\mathrm{OCH}_{2} \mathrm{C}_{6} \mathrm{H}_{4} \mathrm{OCH}_{3}\right), 3.92(\mathrm{~m}, 1 \mathrm{H}, \mathrm{H}-6)$, 3.80 (m, 8H, H-3, H-5, H-C $\left.\mathrm{H}_{4} \mathrm{OCH}_{3}\right), 3.29$ (s, 3H, H-OCH $2 \mathrm{OCH}_{3}$ ), 2.62 (m, 1H, H-2), 2.51 (m, 1H, H-5), 2.39 (m, 1H, H-5'), 1.98 (m, 2H, H-4), 1.71 (m, 6H, H-1', H-6'), 1.62 (s, 3H, H-3'), 1.13 (d, 3H, H-8, J8,2 = 6.8 Hz). 


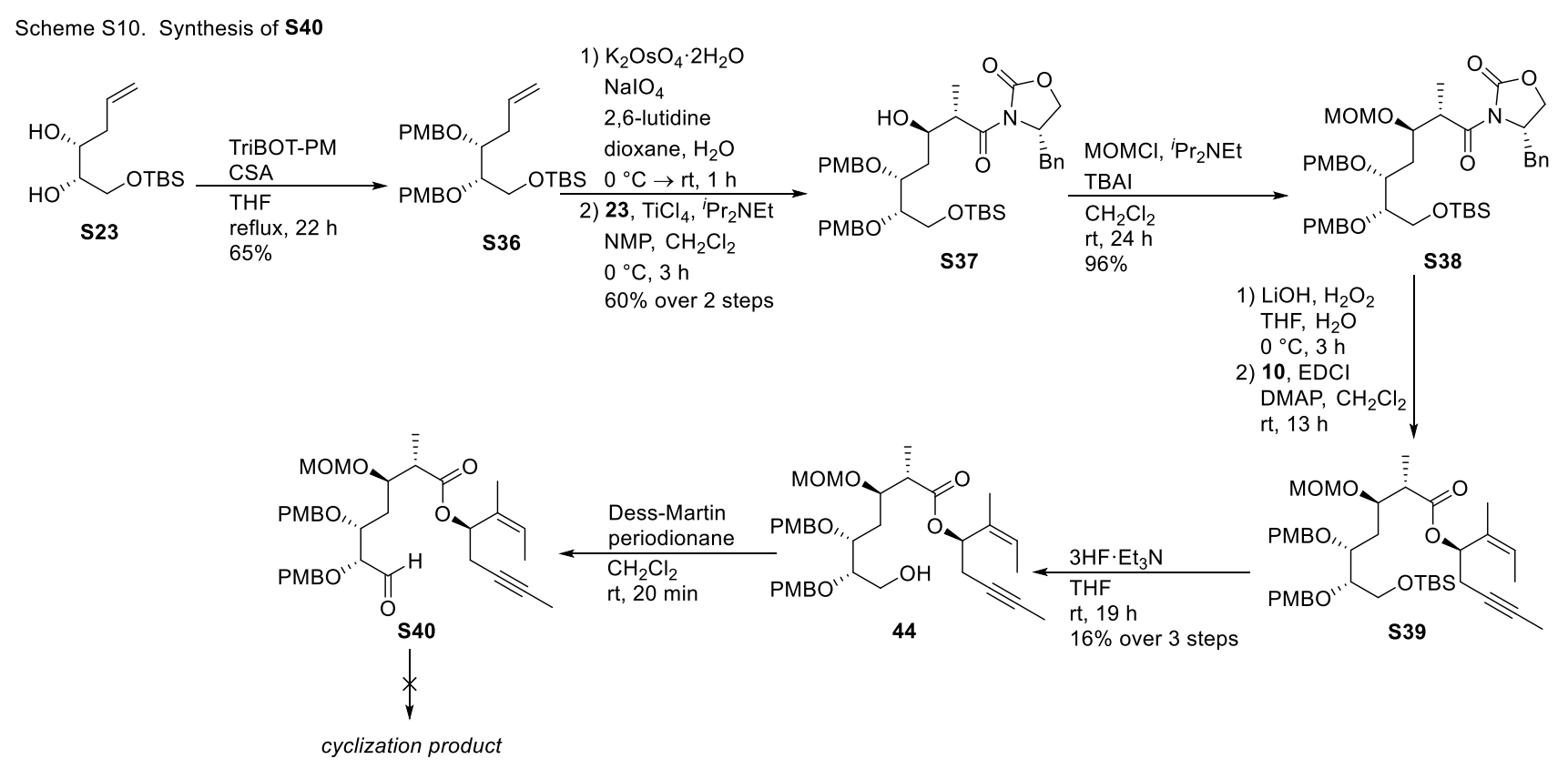

\section{(2S,3R)-1-tert-Butyldimethylsilyloxy-2,3-bis(4-methoxybenzyloxy)hex-5-ene (S36)}

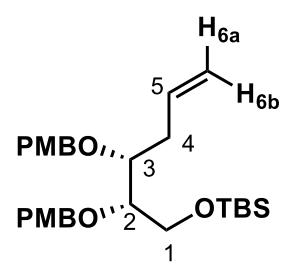

A solution of S23 (1.0 g, $4.06 \mathrm{mmol})$ in THF $(50 \mathrm{~mL})$ was treated with CSA (93 mg, 0.40 mmol) and TriBOT-PM (3.97 g, $8.12 \mathrm{mmol})$ at room temperature. The mixture was warmed to $60{ }^{\circ} \mathrm{C}$ and stirred for $22 \mathrm{~h}$. The mixture was partitioned between EtOAc and $\mathrm{H}_{2} \mathrm{O}$. The organic phase was washed with $1 \mathrm{M}$ aq. $\mathrm{HCl}$, sat. aq. $\mathrm{NaHCO}_{3}, \mathrm{H}_{2} \mathrm{O}$ and brine, dried $\left(\mathrm{Na}_{2} \mathrm{SO}_{4}\right)$, filtered and concentrated in vacuo to afford $\mathbf{S 3 6}(1.28 \mathrm{~g}, 2.65 \mathrm{mmol}, 65 \%)$ as a colorless oil. ${ }^{1} \mathrm{H}$ NMR $\left(\mathrm{CDCl}_{3}, 400 \mathrm{MHz}\right) \delta 7.23\left(\mathrm{~m}, 4 \mathrm{H}, \mathrm{H}-\mathrm{C}_{6} H_{4} \mathrm{OCH}_{3}\right), 6.86\left(\mathrm{~m}, 4 \mathrm{H}, \mathrm{H}-\mathrm{C}_{6} \mathrm{H}_{4} \mathrm{OCH}_{3}\right), 5.85\left(\mathrm{ddt}, 1 \mathrm{H}, \mathrm{H}-5, J_{5,6 \mathrm{~b}}=17.4, J_{5,6 \mathrm{a}}\right.$ $\left.=10.6, J_{5,4}=6.8 \mathrm{~Hz}\right), 5.10\left(\mathrm{dd}, 1 \mathrm{H}, \mathrm{H}-6 \mathrm{~b}, J_{6 \mathrm{~b}, 5}=17.4, J_{g e m}=1.8 \mathrm{~Hz}\right), 5.05\left(\mathrm{dd}, 1 \mathrm{H}, \mathrm{H}-6 \mathrm{a}, J_{6 \mathrm{a}, 5}=10.6, J_{g e m}=1.8 \mathrm{~Hz}\right)$, $4.66\left(\mathrm{~d}, 1 \mathrm{H}, \mathrm{H}-\mathrm{OCH}_{2} \mathrm{C}_{6} \mathrm{H}_{4} \mathrm{OCH}_{3}, J_{\text {gem }}=11.4 \mathrm{~Hz}\right), 4.55\left(\mathrm{~d}, 1 \mathrm{H}, \mathrm{H}-\mathrm{OCH}_{2} \mathrm{C}_{6} \mathrm{H}_{4} \mathrm{OCH}_{3}, J_{\text {gem }}=11.4 \mathrm{~Hz}\right), 4.49(\mathrm{~s}, 2 \mathrm{H}, \mathrm{H}-$ $\left.\mathrm{OCH}_{2} \mathrm{C}_{6} \mathrm{H}_{4} \mathrm{OCH}_{3}\right), 3.80\left(\mathrm{~s}, 6 \mathrm{H}, \mathrm{H}-\mathrm{C}_{6} \mathrm{H}_{4} \mathrm{OCH} H_{3}\right), 3.78(\mathrm{~m}, 1 \mathrm{H}, \mathrm{H}-1), 3.72\left(\mathrm{dd}, 1 \mathrm{H}, \mathrm{H}-1, J_{\text {gem }}=11.0, J_{1,2}=6.0 \mathrm{~Hz}\right), 3.60$ $\left(\mathrm{dd}, 1 \mathrm{H}, \mathrm{H}-3, J_{3,4}=11.4, J_{3,4}=6.8, J_{3,2}=5.2 \mathrm{~Hz}\right), 3.52\left(\mathrm{dd}, 1 \mathrm{H}, \mathrm{H}-2, J_{2,1}=9.6, J_{2,1}=6.0, J_{2,3}=5.2 \mathrm{~Hz}\right), 2.41(\mathrm{dd}, 1 \mathrm{H}$, $\left.\mathrm{H}-4, J_{4,5}=J_{4,3}=6.8 \mathrm{~Hz}\right), 2.39(\mathrm{~m}, 1 \mathrm{H}, \mathrm{H}-4), 0.90\left(\mathrm{~s}, 9 \mathrm{H}, \mathrm{H}-{ }^{t} \mathrm{Bu}\right), 0.05\left(\mathrm{~s}, 6 \mathrm{H}, \mathrm{H}-\mathrm{SiCH}_{3}\right) ;{ }^{13} \mathrm{C} \mathrm{NMR}\left(\mathrm{CDCl}_{3}, 100 \mathrm{MHz}\right)$ $\delta 159.2,135.6,131.1,129.6,117.0,113.8,80.2,72.5,72.0,63.0,55.4,35.1,26.1,18.4,-5.2 ;$ ESIMS-LR $m / z$ 509 $\left[(\mathrm{M}+\mathrm{Na})^{+}\right]$; HRMS (ESI) m/z: $\left[(\mathrm{M}+\mathrm{Na})^{+}\right]$Calcd for $\mathrm{C}_{28} \mathrm{H}_{42} \mathrm{O}_{5} \mathrm{NaSi} 509.2699$; Found 509.2695; $[\alpha]^{20}{ }_{\mathrm{D}}+1.86(c 1.30$, $\left.\mathrm{CHCl}_{3}\right)$. 


\section{(S)-4-Benzyl-3-\{(2S,3R,5R,6S)-7-tert-butyldimethylsilyloxy-3-hydroxy-5,6-bis(4-methoxybenzyloxy)-2-}

methylheptanoyl\}oxazolidin-2-one (S37)

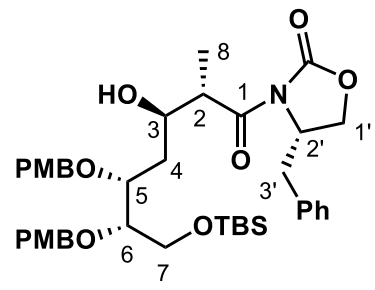

A solution of $\mathbf{S 3 6}(1.28 \mathrm{~g}, 2.63 \mathrm{mmol})$ in dioxane $(20 \mathrm{~mL})$ and $\mathrm{H}_{2} \mathrm{O}(7 \mathrm{~mL})$ was treated with 2,6-lutidine ( $609 \mu \mathrm{L}, 5.26 \mathrm{mmol}), \mathrm{K}_{2} \mathrm{OsO}_{4} \cdot 2 \mathrm{H}_{2} \mathrm{O}(20 \mathrm{mg}, 0.053 \mathrm{mmol})$ and $\mathrm{NaIO}_{4}$ $(2.25 \mathrm{~g}, 10.5 \mathrm{mmol})$ at $0{ }^{\circ} \mathrm{C}$. The mixture was warmed to room temperture and stirred for $1 \mathrm{~h}$. The mixture was partitioned between $\mathrm{CH}_{2} \mathrm{Cl}_{2}$ and $\mathrm{H}_{2} \mathrm{O}$. The organic phase was washed with $1 \mathrm{M}$ aq. $\mathrm{HCl}$, sat. aq. $\mathrm{NaHCO}_{3}, \mathrm{H}_{2} \mathrm{O}$ and brine, dried $\left(\mathrm{Na}_{2} \mathrm{SO}_{4}\right)$, filtered and concentrated in vacuo. The residue was purified by hi-flash silica gel column chromatography $(\phi 2.6 \times 10 \mathrm{~cm}, 2 \rightarrow 20 \%$ EtOAc/hexane) to afford a crude

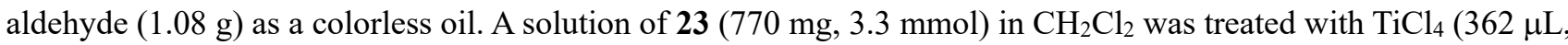
$3.3 \mathrm{mmol})$ at $0{ }^{\circ} \mathrm{C}$ for $15 \mathrm{~min} . N, N$-Diisopropylethylamine $(575 \mu \mathrm{L}, 3.3 \mathrm{mmol})$ was added to the mixture, which was stirred for $20 \mathrm{~min}$. $N$-Methylpyrrolidinone $(312 \mu \mathrm{L}, 3.3 \mathrm{mmol})$ was added to the mixture, which was stirred for 10 min. A solution of the crude aldehyde obtained above (1.08 $\mathrm{g})$ in $\mathrm{CH}_{2} \mathrm{Cl}_{2}$ was added to the mixture via cannula and the whole mixture was stirred for $3 \mathrm{~h}$. The reaction was quenched with sat. aq. $\mathrm{NH}_{4} \mathrm{Cl}$. The organic phase was washed with $1 \mathrm{M}$ aq. $\mathrm{HCl}$, sat. aq. $\mathrm{NaHCO}_{3}, \mathrm{H}_{2} \mathrm{O}$ and brine, dried $\left(\mathrm{Na}_{2} \mathrm{SO}_{4}\right)$, filtered and concentrated in vacuo. The residue was purified by hi-flash silica gel column chromatography $(\phi 2.6 \times 10 \mathrm{~cm}, 10 \rightarrow 30 \%$ EtOAc/hexane) to afford $\mathbf{S 3 7}$ (1.15 g, $1.59 \mathrm{mmol}, 60 \%$ over 2 steps) as a colorless oil. ${ }^{1} \mathrm{H} \mathrm{NMR}\left(\mathrm{CDCl}_{3}, 400 \mathrm{MHz}\right) \delta 7.26\left(\mathrm{~m}, 9 \mathrm{H}, \mathrm{H}-\mathrm{C}_{6} \mathrm{H}_{4} \mathrm{OCH}\right.$, $\mathrm{H}-\mathrm{Ph}), 6.83\left(\mathrm{~m}, 4 \mathrm{H}, \mathrm{H}-\mathrm{C}_{6} H_{4} \mathrm{OCH}_{3}\right), 4.62\left(\mathrm{~m}, 1 \mathrm{H}, \mathrm{H}-2^{2}\right), 4.61\left(\mathrm{~m}, 2 \mathrm{H}, \mathrm{H}-\mathrm{OCH}_{2} \mathrm{C}_{6} \mathrm{H}_{4} \mathrm{OCH}_{3}\right), 4.57(\mathrm{~d}, 1 \mathrm{H}, \mathrm{H}-$ $\left.\mathrm{OCH}_{2} \mathrm{C}_{6} \mathrm{H}_{4} \mathrm{OCH}_{3}, J_{\text {gem }}=11.0 \mathrm{~Hz}\right), 4.48\left(\mathrm{~d}, 1 \mathrm{H}, \mathrm{H}-\mathrm{OCH}_{2} \mathrm{C}_{6} \mathrm{H}_{4} \mathrm{OCH}_{3}, J_{\text {gem }}=11.0 \mathrm{~Hz}\right), 4.19(\mathrm{~m}, 2 \mathrm{H}, \mathrm{H}-2, \mathrm{H}-3), 4.14$ (m, 2H, H-3'), 3.86 (dq, 1H, H-5, J5,4 = 3.6, $\left.J_{5,6}=7.8 \mathrm{~Hz}\right), 3.78$ (s, 6H, H-C $\left.\mathrm{C}_{6} \mathrm{H}_{4} \mathrm{OCH} \mathrm{H}_{3}\right), 3.62$ (m, 3H, H-6, H-7), 3.24 $\left(\mathrm{dd}, 1 \mathrm{H}, \mathrm{H}-1^{\prime}, J_{1^{\prime}, 2^{\prime}}=9.6, J_{g e m}=13.4 \mathrm{~Hz}\right), 3.20(\mathrm{br} \mathrm{s}, 1 \mathrm{H}, \mathrm{H}-\mathrm{OH}), 2.75\left(\mathrm{dd}, 1 \mathrm{H}, \mathrm{H}-1^{\prime}, J_{1^{\prime}, 2^{\prime}}=9.6, J_{g e m}=13.4 \mathrm{~Hz}\right), 1.71$

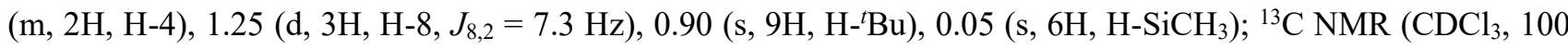
MHz) $\delta 177.2,159.3,153.1,135.3,131.5,129.7,129.1,127.5,113.9,80.8,72.6,72.3,68.8,66.2,63.0,55.4,43.0$, 37.9, 34.8, 26.1, 18.4, 11.5, -5.2; ESIMS-LR $m / z 744\left[(\mathrm{M}+\mathrm{Na})^{+}\right]$; HRMS (ESI) $\mathrm{m} / \mathrm{z}:\left[(\mathrm{M}+\mathrm{Na})^{+}\right]$Calcd for $\mathrm{C}_{40} \mathrm{H}_{55} \mathrm{NO}_{9} \mathrm{NaSi}$ 744.3544; Found 744.3543; $[\alpha]^{20}{ }_{\mathrm{D}}+40.9\left(c \quad 0.82, \mathrm{CHCl}_{3}\right)$.

\section{(S)-4-Benzyl-3[(2S,3R,5R,6S)-7-tert-butyldimethylsilyloxy-5,6-bis(4-methoxybenzyloxy)-3-methoxymethoxy-}

\section{2-methylheptanoyl]oxazolidin-2-one (S38)}

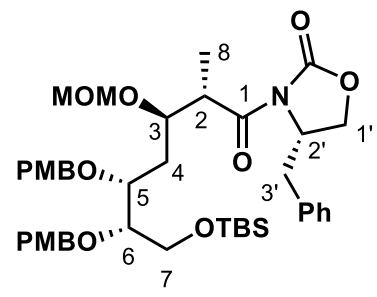

A solution of $\mathbf{S 3 7}(1.13 \mathrm{~g}, 1.56 \mathrm{mmol})$ in $\mathrm{CH}_{2} \mathrm{Cl}_{2}(13 \mathrm{~mL})$ was treated with ${ }^{i} \mathrm{Pr}_{2} \mathrm{NEt}$ $(2.17 \mathrm{~mL}, 12.48 \mathrm{mmol}), \mathrm{TBAI}(58 \mathrm{mg}, 0.156 \mathrm{mmol})$ and $\mathrm{MOMCl}(474 \mu \mathrm{L}, 6.24 \mathrm{mmol})$ at room temperature for $24 \mathrm{~h}$. The reaction was quenched with $\mathrm{H}_{2} \mathrm{O}$, and the mixture was partitioned between EtOAc and $\mathrm{H}_{2} \mathrm{O}$. The organic phase was washed with $1 \mathrm{Maq}$. $\mathrm{HCl}$, sat. aq. $\mathrm{NaHCO}_{3}, \mathrm{H}_{2} \mathrm{O}$ and brine, dried $\left(\mathrm{Na}_{2} \mathrm{SO}_{4}\right)$, filtered and concentrated in vacuo to afford $\mathbf{S 3 8}(1.15 \mathrm{~g}, 1.5 \mathrm{mmol}, 96 \%)$ as a colorless oil. ${ }^{1} \mathrm{H} \mathrm{NMR}\left(\mathrm{CDCl}_{3}, 400 \mathrm{MHz}\right) \delta 7.26\left(\mathrm{~m}, 9 \mathrm{H}, \mathrm{H}-\mathrm{C}_{6} \mathrm{H}_{4} \mathrm{OCH}_{3}, \mathrm{H}-\right.$ 
$\mathrm{Ph}), 6.84\left(\mathrm{~m}, 4 \mathrm{H}, \mathrm{H}-\mathrm{C}_{6} \mathrm{H}_{4} \mathrm{OCH}_{3}\right), 4.63$ (m, 1H, H-2'), 4.55 (m, 6H, H-OCH $\left.\mathrm{C}_{6} \mathrm{H}_{4} \mathrm{OCH}_{3}, \mathrm{H}-\mathrm{OCH} \mathrm{H}_{2} \mathrm{O}\right), 4.10$ (m, 4H, H2, H-3, H-3'), 3.77 (m, 10H, H-5, H-6, H-7, $\mathrm{H}_{-} \mathrm{C}_{6} \mathrm{H}_{4} \mathrm{OCH}_{3}$ ), 3.29 (s, 3H, H-OCH $\mathrm{OCH}_{3}$ ), 3.28 (m, 1H, H-1'), 2.74 $\left(\mathrm{dd}, 1 \mathrm{H}, \mathrm{H}-1^{\prime}, J_{1^{\prime}, 2^{\prime}}=10.1, J_{\text {gem }}=13.3 \mathrm{~Hz}\right), 1.84\left(\mathrm{dd}, 1 \mathrm{H}, \mathrm{H}-4, J_{\text {gem }}=14.6, J_{4,5}=10.6\right), 1.75\left(\mathrm{dd}, 1 \mathrm{H}, \mathrm{H}-4, J_{\text {gem }}=14.6\right.$, $\left.J_{4,5}=10.6 \mathrm{~Hz}\right), 1.21\left(\mathrm{~d}, 3 \mathrm{H}, \mathrm{H}-8, J_{8,2}=7.3 \mathrm{~Hz}\right), 0.88\left(\mathrm{~s}, 9 \mathrm{H}, \mathrm{H}-{ }^{t} \mathrm{Bu}\right), 0.04\left(\mathrm{~s}, 6 \mathrm{H}, \mathrm{H}-\mathrm{SiCH}_{3}\right) ;{ }^{13} \mathrm{C} \mathrm{NMR}\left(\mathrm{CDCl}_{3}, 100\right.$ MHz) $\delta 174.5,159.1,153.4,135.7,131.2,129.4,129.0,127.4,113.8,97.2,80.9,76.2,72.6,71.5,66.1,63.1,56.2$, 55.4, 41.9, 37.9, 34.4, 26.1, 18.4, 12.5, -5.1; ESIMS-LR m/z 788 [(M+Na) $)^{+}$; HRMS (ESI) m/z: [(M+Na) $\left.{ }^{+}\right]$Calcd for $\mathrm{C}_{42} \mathrm{H}_{59} \mathrm{NO}_{10} \mathrm{NaSi} 788.3806$; Found 788.3817; $[\alpha]^{20}{ }_{\mathrm{D}}+53.1\left(c \mathrm{c} 0.98, \mathrm{CHCl}_{3}\right)$.

\section{(R,Z)-3-Methyloct-2-en-6-yn-4-yl (2S,3R,5R,6S)-7-hydroxy-5,6-bis(4-methoxybenzyloxy)-3-methoxymethoxy-}

\section{2-methylheptanoate (44)}

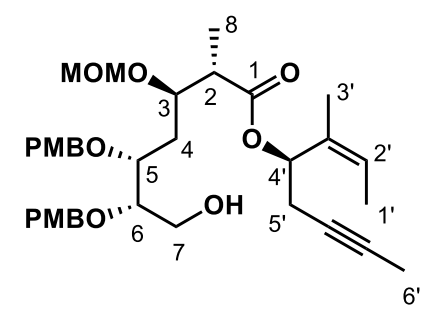

A solution of $\mathbf{S 3 8}(383 \mathrm{mg}, 0.5 \mathrm{mmol})$ in THF $(4 \mathrm{~mL})$ and $\mathrm{H}_{2} \mathrm{O}(1 \mathrm{~mL})$ was treated with $\mathrm{H}_{2} \mathrm{O}_{2}\left(228 \mu \mathrm{L}, 2 \mathrm{mmol}, 30 \%\right.$ in $\left.\mathrm{H}_{2} \mathrm{O}\right)$ and $\mathrm{LiOH}(42 \mathrm{mg}, 1 \mathrm{mmol})$ at $0{ }^{\circ} \mathrm{C}$ for $3 \mathrm{~h}$. The reaction was quenched with sat. aq. $\mathrm{Na}_{2} \mathrm{~S}_{2} \mathrm{O}_{3}$. The mixture was partitioned between $\mathrm{Et}_{2} \mathrm{O}$ and sat. aq. $\mathrm{NaHCO}_{3}$. The organic phase was washed with brine, dried $\left(\mathrm{Na}_{2} \mathrm{SO}_{4}\right)$,

filtered and concentrated in vacuo. A solution of the residue $(100 \mathrm{mg})$ and $\mathbf{1 0}(34 \mathrm{mg}$, $0.246 \mathrm{mmol})$ in $\mathrm{CH}_{2} \mathrm{Cl}_{2}(2 \mathrm{~mL})$ was treated with DMAP (2 $\left.\mathrm{mg}, 0.016 \mathrm{mmol}\right)$ and EDCI (63 $\left.\mathrm{mg}, 0.328 \mathrm{mmol}\right)$ at room temperature for $13 \mathrm{~h}$. The mixture was partitioned between EtOAc and $\mathrm{H}_{2} \mathrm{O}$. The organic phase was washed with 1 $\mathrm{M}$ aq. $\mathrm{HCl}$, sat. aq. $\mathrm{NaHCO}_{3}, \mathrm{H}_{2} \mathrm{O}$ and brine, dried $\left(\mathrm{Na}_{2} \mathrm{SO}_{4}\right)$, filtered and concentrated in vacuo. A solution of the residue $(180 \mathrm{mg})$ in THF $(2 \mathrm{~mL})$ was treated with $3 \mathrm{HF} \cdot \mathrm{Et}_{3} \mathrm{~N}(267 \mu \mathrm{L}, 1.64 \mathrm{mmol})$ at room temperature for $19 \mathrm{~h}$. The mixture was partitioned between EtOAc and $\mathrm{H}_{2} \mathrm{O}$. The organic phase was washed with $1 \mathrm{Maq}$. $\mathrm{HCl}$, sat. aq. $\mathrm{NaHCO}_{3}$, $\mathrm{H}_{2} \mathrm{O}$ and brine, dried $\left(\mathrm{Na}_{2} \mathrm{SO}_{4}\right)$, filtered and concentrated in vacuo. The residue was purified by hi-flash silica gel column chromatography $(\phi 2.0 \times 6.5 \mathrm{~cm}, 20 \rightarrow 35 \rightarrow 50 \%$ EtOAc/hexane) to afford 44 (50 mg, $0.082 \mathrm{mmol}, 16 \%$ over 3 steps) as a colorless oil. ${ }^{1} \mathrm{H}$ NMR $\left(\mathrm{CDCl}_{3}, 400 \mathrm{MHz}\right) \delta 7.29\left(\mathrm{~m}, 4 \mathrm{H}, \mathrm{H}-\mathrm{C}_{6} \mathrm{H}_{4} \mathrm{OCH}_{3}\right), 6.89\left(\mathrm{~m}, 2 \mathrm{H}, \mathrm{H}-\mathrm{C}_{6} \mathrm{H}_{4} \mathrm{OCH}_{3}\right)$, $6.86\left(\mathrm{~m}, 2 \mathrm{H}, \mathrm{H}-\mathrm{C}_{6} H_{4} \mathrm{OCH}_{3}\right), 5.72\left(\mathrm{t}, 1 \mathrm{H}, \mathrm{H}-4^{\prime}, J_{4^{\prime}, 5^{\prime}}=9.6, J_{4^{\prime}, 3^{\prime}}=3.2 \mathrm{~Hz}\right), 5.43\left(\mathrm{q}, 1 \mathrm{H}, \mathrm{H}-2^{\prime}, J_{2^{\prime}, 1^{\prime}}=6.8 \mathrm{~Hz}\right), 4.73(\mathrm{~d}$, $\left.1 \mathrm{H}, \mathrm{H}-\mathrm{OCH}_{2} \mathrm{C}_{6} \mathrm{H}_{4} \mathrm{OCH}_{3}, J_{\text {gem }}=11.0 \mathrm{~Hz}\right), 4.67\left(\mathrm{~d}, 1 \mathrm{H}, \mathrm{H}-\mathrm{OCH}_{2} \mathrm{C}_{6} \mathrm{H}_{4} \mathrm{OCH}_{3}, J_{\text {gem }}=11.9 \mathrm{~Hz}\right), 4.65\left(\mathrm{~d}, 1 \mathrm{H}, \mathrm{H}-\mathrm{OCH} \mathrm{H}_{2} \mathrm{O}\right.$, $\left.J_{\text {gem }}=6.8 \mathrm{~Hz}\right), 4.54\left(\mathrm{~d}, 1 \mathrm{H}, \mathrm{H}-\mathrm{OCH}_{2} \mathrm{C}_{6} \mathrm{H}_{4} \mathrm{OCH}_{3}, J_{\text {gem }}=11.9 \mathrm{~Hz}\right), 4.51\left(\mathrm{~d}, 1 \mathrm{H}, \mathrm{H}-\mathrm{OCH} \mathrm{H}_{2} \mathrm{O}, J_{\text {gem }}=6.8 \mathrm{~Hz}\right), 4.49(\mathrm{~d}, 1 \mathrm{H}$, $\left.\mathrm{H}-\mathrm{OCH}_{2} \mathrm{C}_{6} \mathrm{H}_{4} \mathrm{OCH}_{3}, J_{\text {gem }}=11.0 \mathrm{~Hz}\right), 4.09\left(\mathrm{ddd}, 1 \mathrm{H}, \mathrm{H}-3, J_{3,4}=9.6 \mathrm{~Hz}, J_{3,2}=J_{3,4}=3.2 \mathrm{~Hz}\right), 3.85\left(\mathrm{~d}, 1 \mathrm{H}, \mathrm{H}-5, J_{5,4}=\right.$ $9.6 \mathrm{~Hz}$ ), 3.80 (s, 6H, H-C $\mathrm{H}_{4} \mathrm{OCH}_{3}$ ), 3.72 (m, 2H, H-7), 3.59 (m, 1H, H-6), 3.35 (s, 3H, H-OCH $\mathrm{OCH}_{3}$ ), 2.65 (dq, $1 \mathrm{H}, \mathrm{H}-2, J_{2,8}=7.3 \mathrm{~Hz}, J_{2,3}=3.2 \mathrm{~Hz}$ ), 2.54 (ddd, $1 \mathrm{H}, \mathrm{H}-5^{\prime}, J_{\text {gem }}=17.0, J_{5^{\prime}, 4^{\prime}}=7.3, J_{5^{\prime}, 6^{\prime}}=2.8 \mathrm{~Hz}$ ), 2.40 (ddd, $1 \mathrm{H}, \mathrm{H}-5^{\prime}$, $\left.J_{\text {gem }}=17.0, J_{5^{\prime}, 4^{\prime}}=7.3, J_{5^{\prime}, 6^{\prime}}=2.3 \mathrm{~Hz}\right), 2.24\left(\mathrm{dd}, 1 \mathrm{H}, \mathrm{H}-\mathrm{OH}, J_{\mathrm{OH}, 7}=7.3, J_{\mathrm{OH}, 7}=5.0 \mathrm{~Hz}\right), 1.78(\mathrm{~m}, 2 \mathrm{H}, \mathrm{H}-4), 1.72(\mathrm{~m}$,

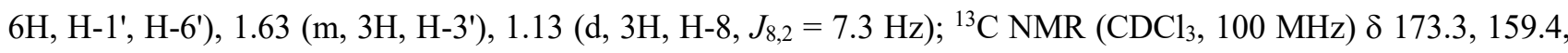
$132.4,130.6,130.4,129.6,124.7,114.0,97.4,81.0,74.9$, 73.0, 72.0, 71.0, 61.6, 56.0, 55.4, 44.5, 35.6, 23.0, 17.9, 13.3, 11.4, 3.6; ESIMS-LR $m / z 635\left[(\mathrm{M}+\mathrm{Na})^{+}\right]$; HRMS (ESI) m/z: $\left[(\mathrm{M}+\mathrm{Na})^{+}\right]$Calcd for $\mathrm{C}_{35} \mathrm{H}_{48} \mathrm{O}{ }_{9} \mathrm{Na} 635.3196$; 


\section{(R,Z)-3-Methyloct-2-en-6-yn-4-yl (2S,3R,5R,6R)-5,6-bis(4-methoxybenzyloxy)-3-methoxymethoxy-2-} methyl-7-oxoheptanoate (S40)

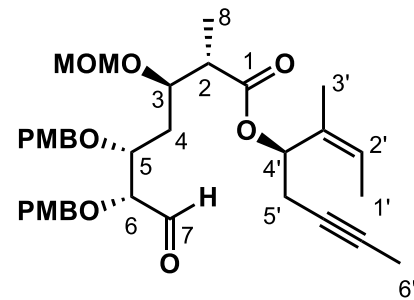

A solution of $44(11 \mathrm{mg}, 0.018 \mathrm{mmol})$ in $\mathrm{CH}_{2} \mathrm{Cl}_{2}(1 \mathrm{~mL})$ was treated with DessMartin periodinane (15 $\mathrm{mg}, 0.03 \mathrm{mmol}$ ) at room temperature for $20 \mathrm{~min}$. The reaction was quenched with sat. aq. $\mathrm{Na}_{2} \mathrm{~S}_{2} \mathrm{O}_{3}$ and sat. aq. $\mathrm{NaHCO}_{3}$. The mixture was partitioned between EtOAc and $\mathrm{H}_{2} \mathrm{O}$. The organic phase was washed with brine, dried $\left(\mathrm{Na}_{2} \mathrm{SO}_{4}\right)$, filtered and concentrated in vacuo. The residue was purified by silica gel column chromatography ( $\phi 1 \times 8 \mathrm{~cm}, 33 \%$ EtOAc/hexane) to afford a crude $\mathbf{S 4 0}(11 \mathrm{mg})$ as a colorless oil. The crude S40 was used for the next reaction without further purification. ${ }^{1} \mathrm{H} \mathrm{NMR}\left(\mathrm{CDCl}_{3}, 400 \mathrm{MHz}\right) \delta 9.68(\mathrm{~s}, 1 \mathrm{H}, \mathrm{CHO})$, 7.25 (m, 4H, H-C $\left.\mathrm{C}_{6} \mathrm{H}_{4} \mathrm{OCH}_{3}\right), 6.86\left(\mathrm{~m}, 4 \mathrm{H}, \mathrm{H}-\mathrm{C}_{6} H_{4} \mathrm{OCH}_{3}\right), 5.70\left(\mathrm{t}, 1 \mathrm{H}, \mathrm{H}-4^{\prime}, J_{4^{\prime} 5^{\prime}}=J_{4^{\prime}, 5^{\prime}}=7.0 \mathrm{~Hz}\right), 5.43\left(\mathrm{q}, 1 \mathrm{H}, \mathrm{H}-2^{\prime}\right.$, $\left.J_{2^{\prime}, 1^{\prime}}=6.6 \mathrm{~Hz}\right), 4.48-4.60\left(\mathrm{~m}, 6 \mathrm{H}, \mathrm{H}-\mathrm{OCH}_{2} \mathrm{O}, \mathrm{H}-\mathrm{OCH}_{2} \mathrm{C}_{6} \mathrm{H}_{4} \mathrm{OCH}_{3}\right), 4.47(\mathrm{~m}, 1 \mathrm{H}, \mathrm{H}-6), 4.09$ (m, 1H, H-3), 3.96 (m, 1H, H-5), 3.80 (s, 6H, H-C $6 \mathrm{H}_{4} \mathrm{OCH}_{3}$ ), 3.32 (s, 3H, H-OCH $\mathrm{OCH}_{3}$ ), 2.62 (m, 1H, H-2), 2.51 (m, 1H, H-5'), 2.39 (m, 1H, H-5'), 1.86 (m, 2H, H-4), 1.71 (m, 6H, H-1', H-6'), 1.62 (s, 3H, H-3'), 1.13 (d, 3H, H-8, J8,2 = 7.3 Hz). 

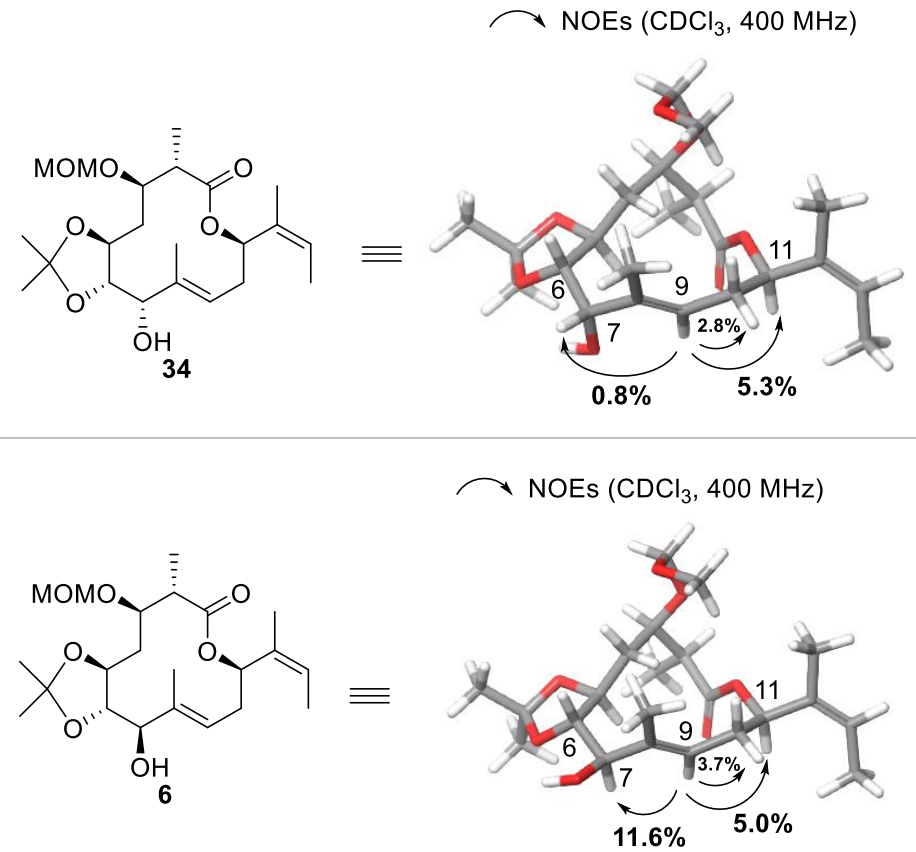

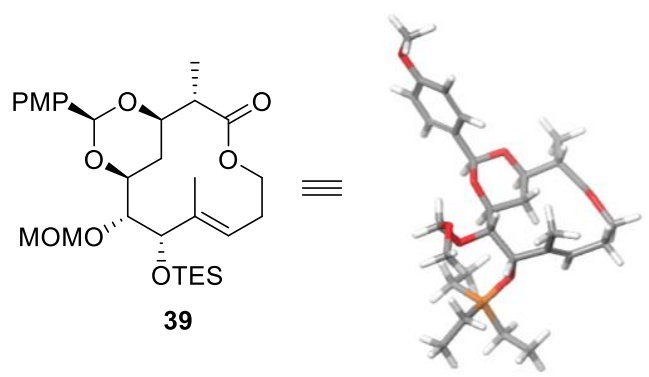

$0 \mathrm{~kJ} / \mathrm{mol}^{*}$

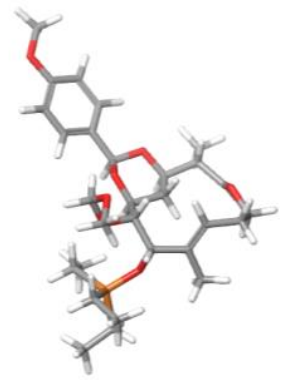

$+23 \mathrm{~kJ} / \mathrm{mol}^{*}$

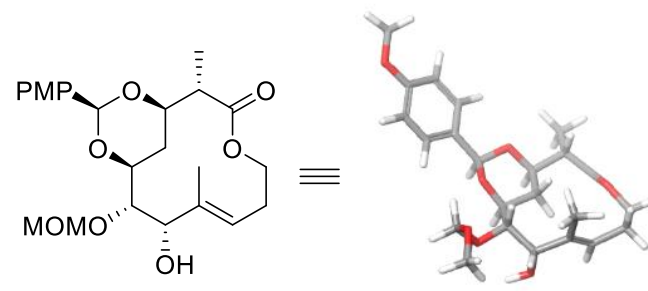

$0 \mathrm{~kJ} / \mathrm{mol}^{*}$

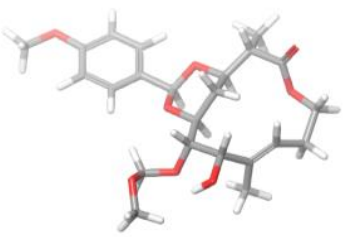

$+9.1 \mathrm{~kJ} / \mathrm{mol}^{*}$

Figure S1. The initial structure of each compound was generated manually, then conformational search was carried out by MacroModel using OPLS-2005 force field. A pool of conformers was generated for each ligand with MCMM conformational algorithm, by accepting all conformers with energies up to $21.0 \mathrm{~kJ} / \mathrm{mol}$ from the global minimum. The maximum numberof Monte Carlo steps was set to 1000. For compound $\mathbf{3 4}$ and $\mathbf{6}$, the global minimum structure was selected for further analysis (Figure S1). For compound $\mathbf{3 9}$ and S17, the global minimum and second lowest local minimum structure were selected. *relative potential energy 
${ }^{1} \mathrm{H} \mathrm{NMR}\left(\mathrm{CDCl}_{3}, 400 \mathrm{MHz}\right)$

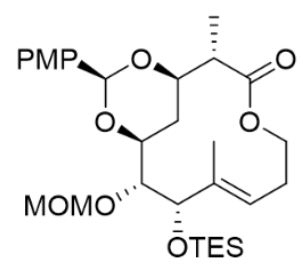
$25^{\circ} \mathrm{C}$

39

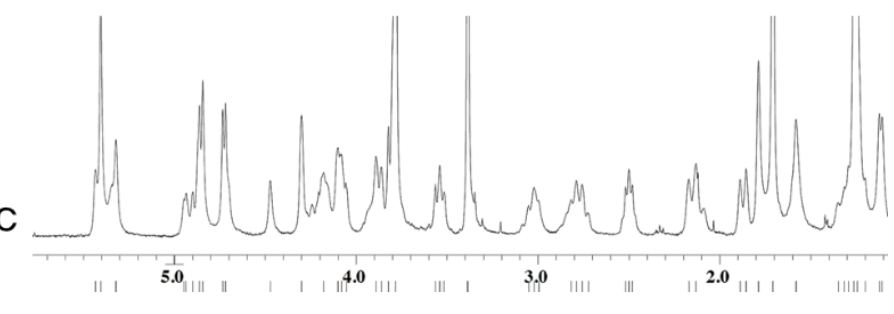

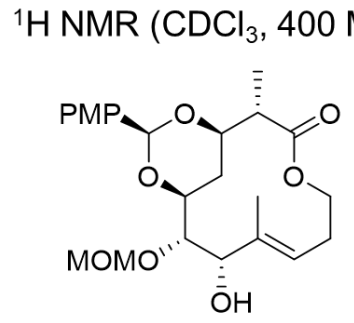

S17
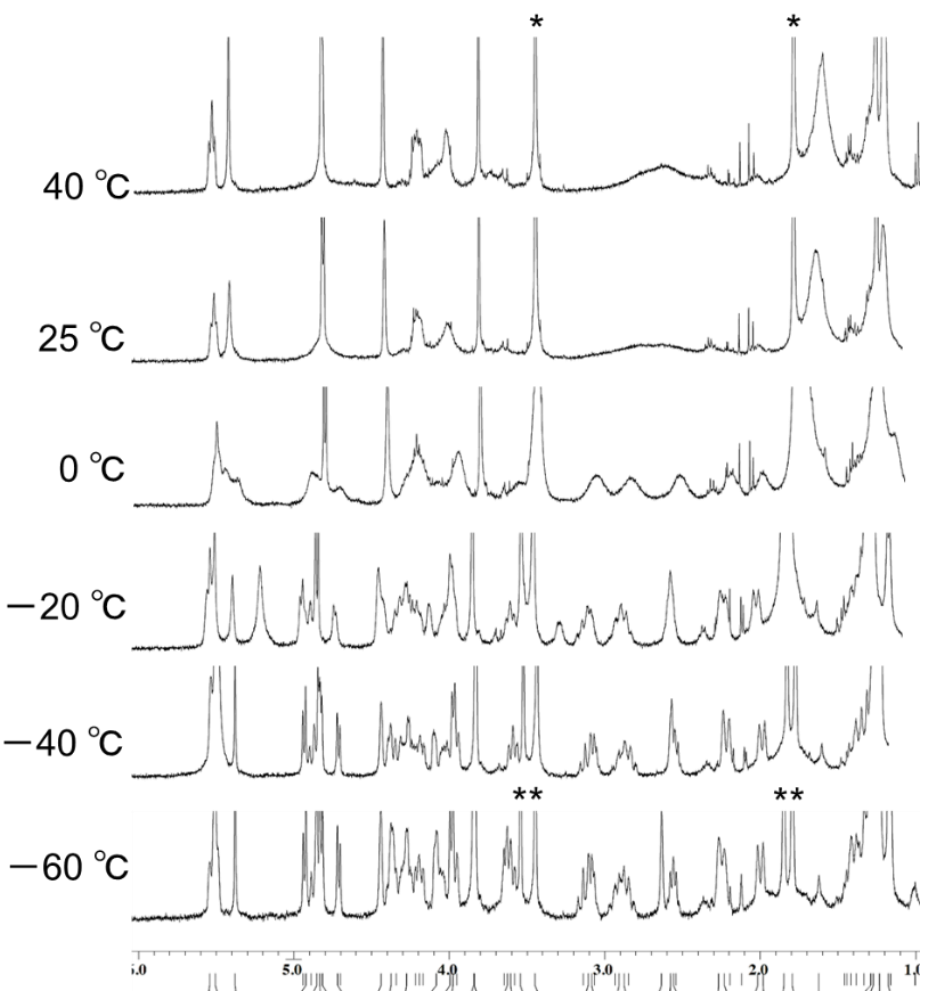

Figure S2. ${ }^{1} \mathrm{H}$ NMR spectra of $\mathbf{3 9}$ and $\mathbf{S 1 7}$ was measured in $\mathrm{CDCl}_{3}$ solution using JEOL ECX400P. Conformer of S17 was detected by variable temperature ${ }^{1} \mathrm{H}$ NMR analysis $\left(40{ }^{\circ} \mathrm{C}, 25{ }^{\circ} \mathrm{C}, 0{ }^{\circ} \mathrm{C},-20{ }^{\circ} \mathrm{C},-40{ }^{\circ} \mathrm{C}\right.$ and $\left.-60{ }^{\circ} \mathrm{C}\right)$. 


\section{References}

S1) Speltz, T. E.; Fanning, S. W.; Mayne, C. G.; Fowler, C.; Tajkhorshid, E.; Greene, G. L.; Moore, T. W. Angew. Chem. Int. Ed. 2016, 5, 4252-4255.

S2) Hilpert, L. J.; Sieger, S. V.; Haydl, A. M.; Breit, B. Angew. Chem. Int. Ed. 2019, 58, 3378-3381.

S3) Fujino, H.; Nagatomo, M.; Paudel, A.; Panthee, S.; Hamamoto, H.; Sekimizu, K.; Inoue, M. Angew. Chem. Int. Ed. 2017, 56, 11865-11869.

S4) Liu, J. H.; Long, Y. Q. Tetrahedron Lett. 2009, 50, 4592-4594.

S5) Dias, L. C.; Ferreira, M. A. B. J. Org. Chem. 2012, 77, 4046-4062.

S6) Kwon, Y.; Schulthoff, S.; Dao, Q. M.; Wirtz, C.; Fürstner A. Chem. Eur. J. 2018, 24, 109-114.

S7) Dureau, R.; Legentil, L.; Daniellou, R.; Ferrières, V. J. Org. Chem. 2012, 77, 1301-1307.

S8) Gurjar, M. K.; Reddy, L. K.; Hotha, S. J. Org. Chem. 2001, 66, 4657-4660.

S9) Urosa, A.; Marcos, I. S.; Díez, D.; Padrón, J.; Basabe, P. J. Org. Chem. 2015, 80, 6447-6455.

S10) Bolte, B.; Basutto, J. A.; Bryan, C. S.; Garson, M. J.; Banwell, M. G.; Ward, J. S. J. Org. Chem. 2015, 80, 460-470. 


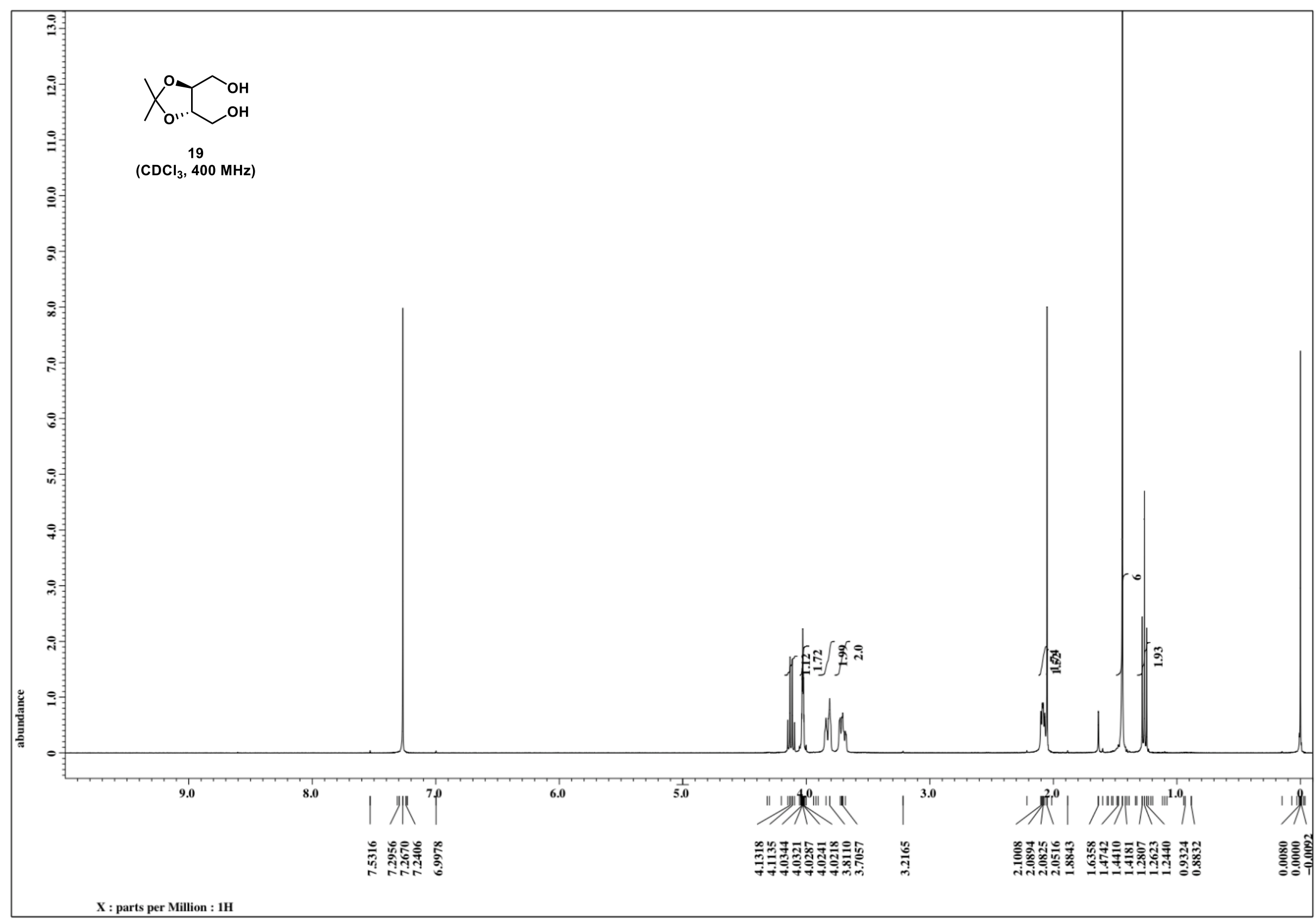




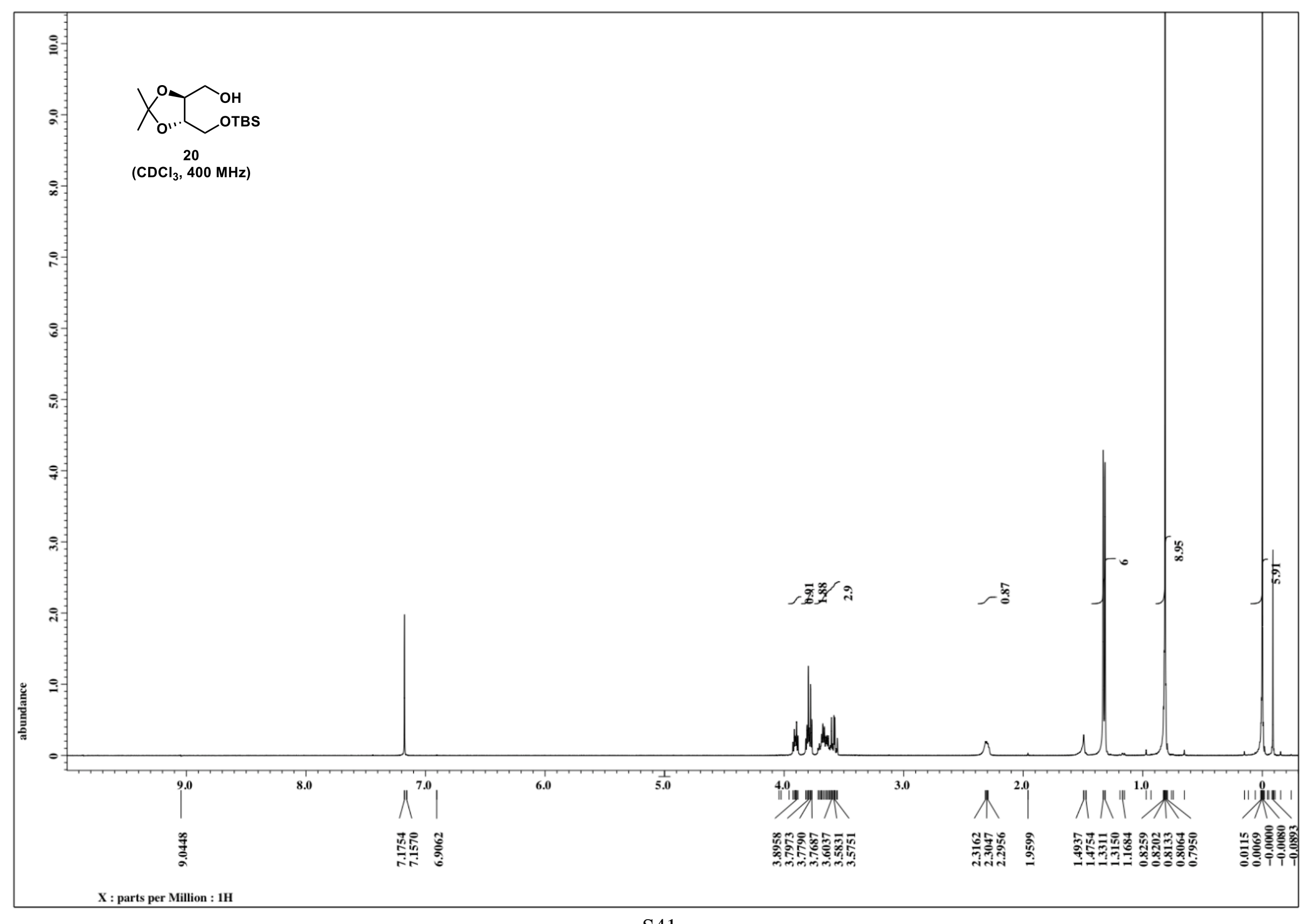




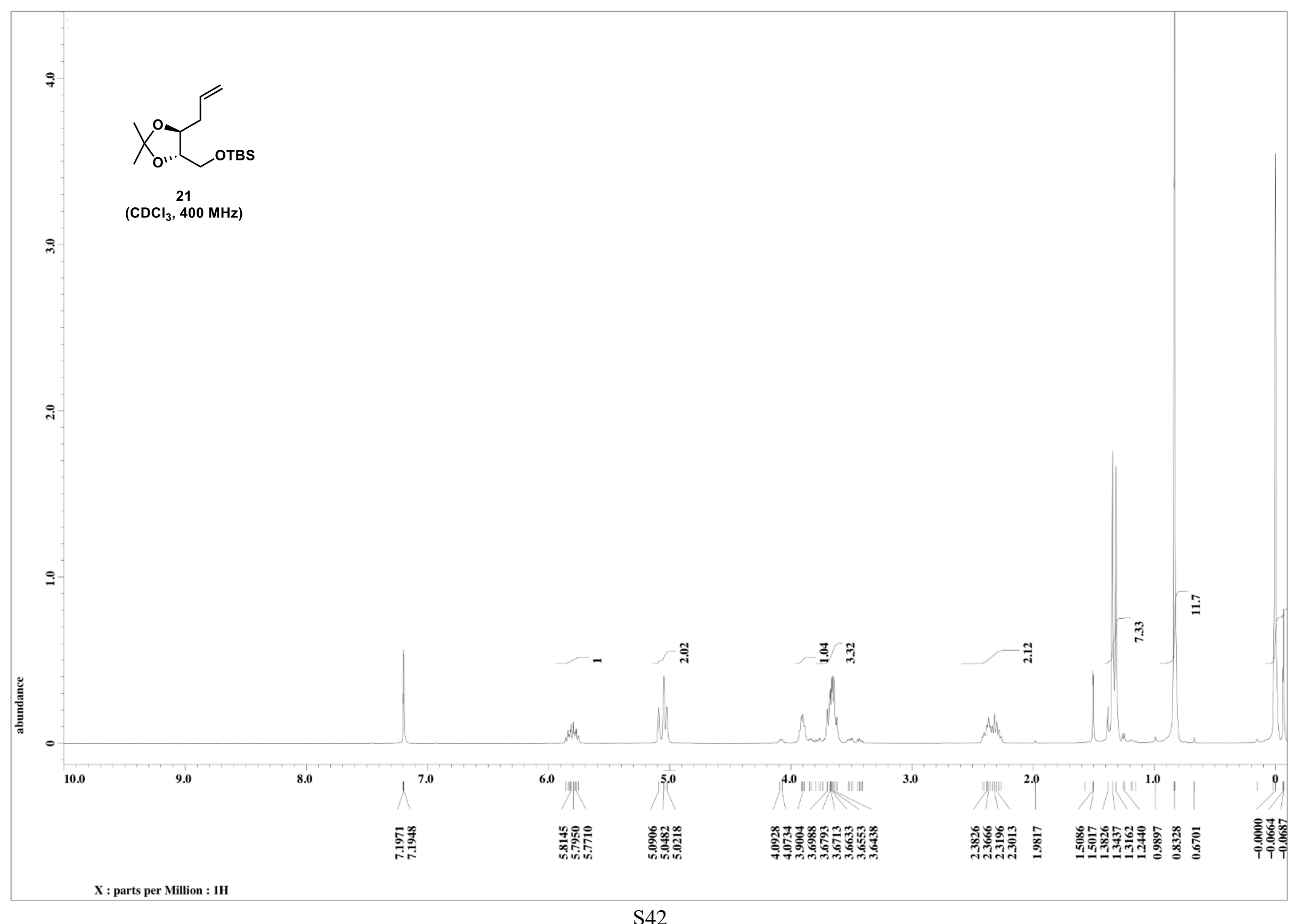




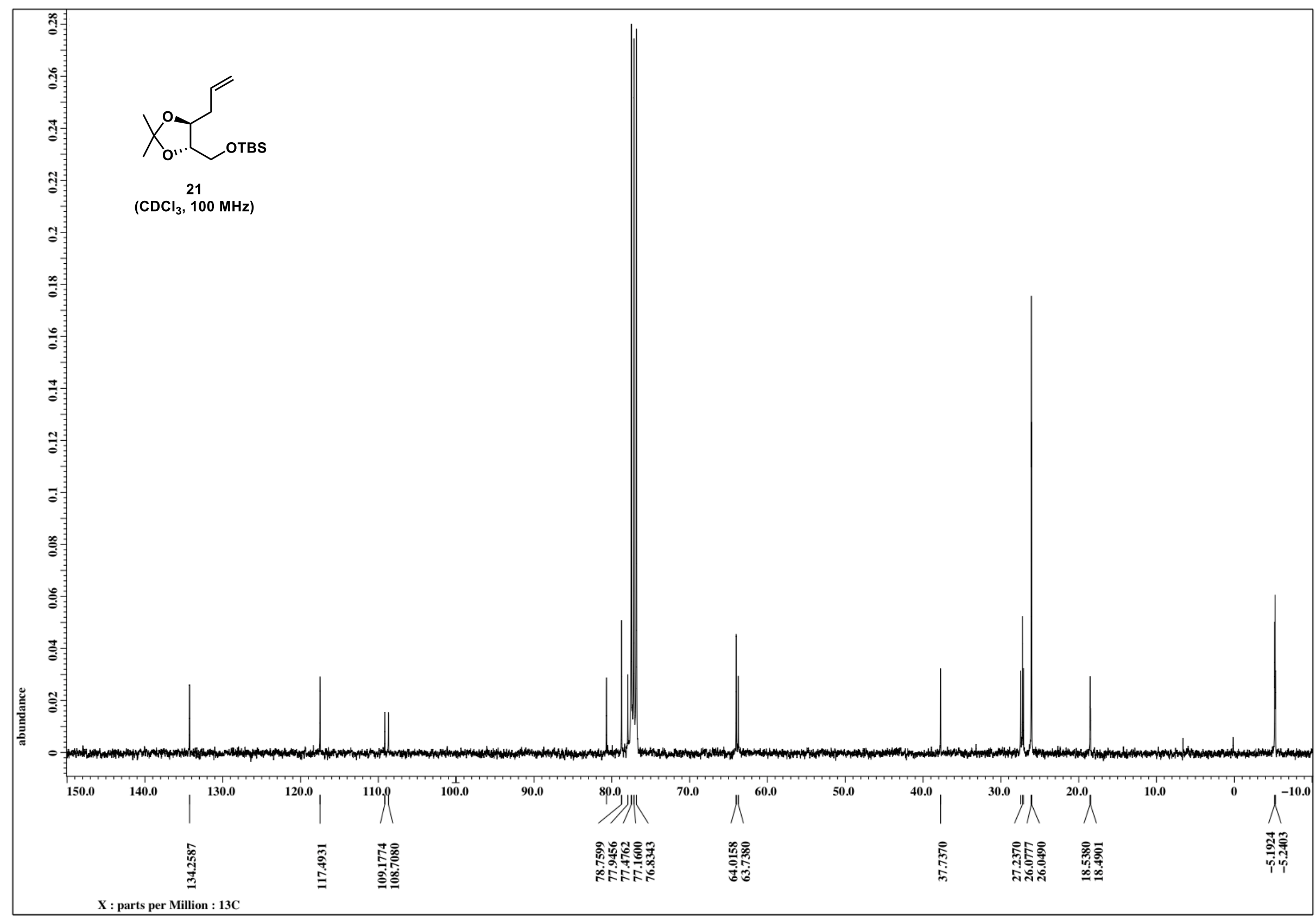




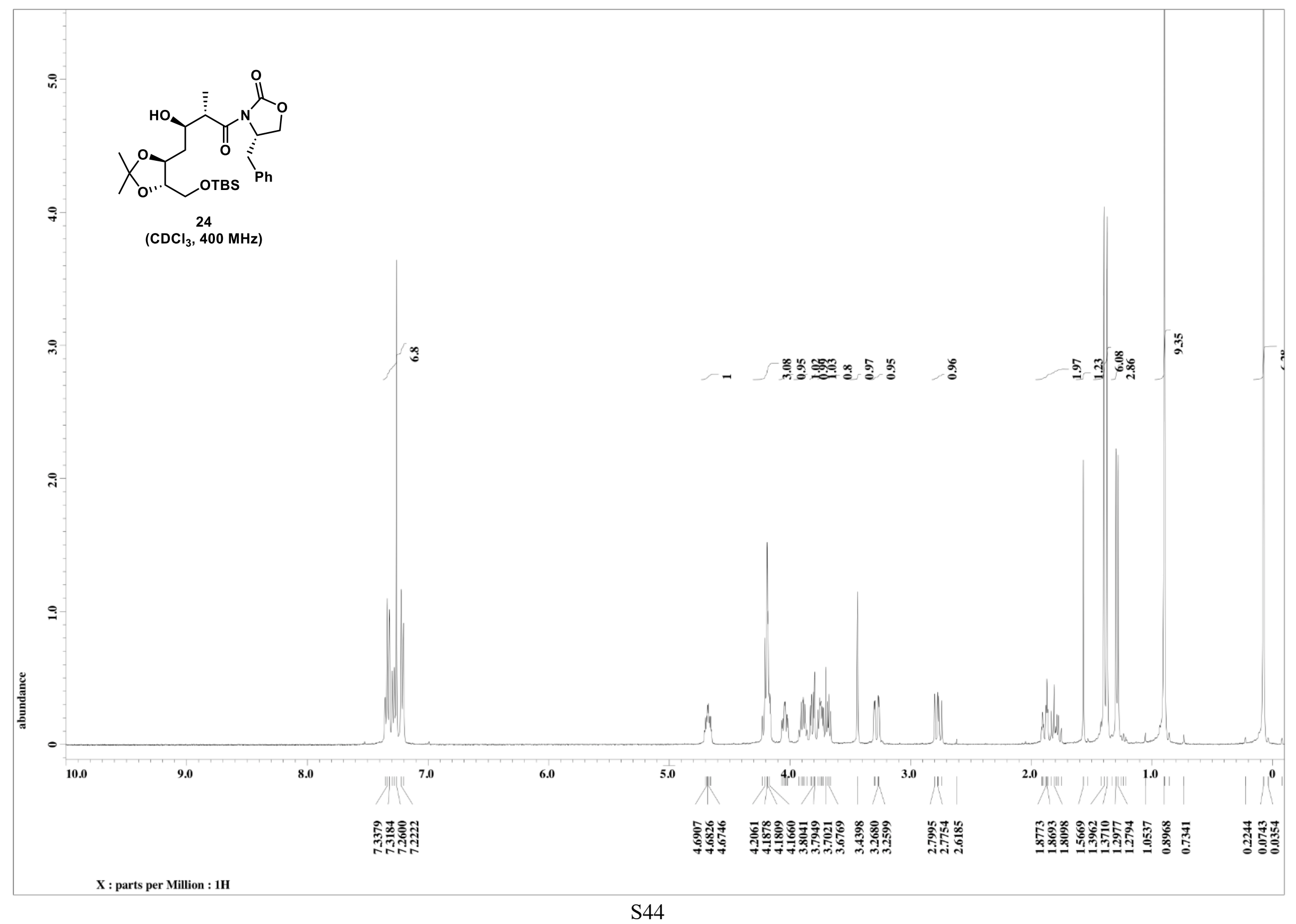




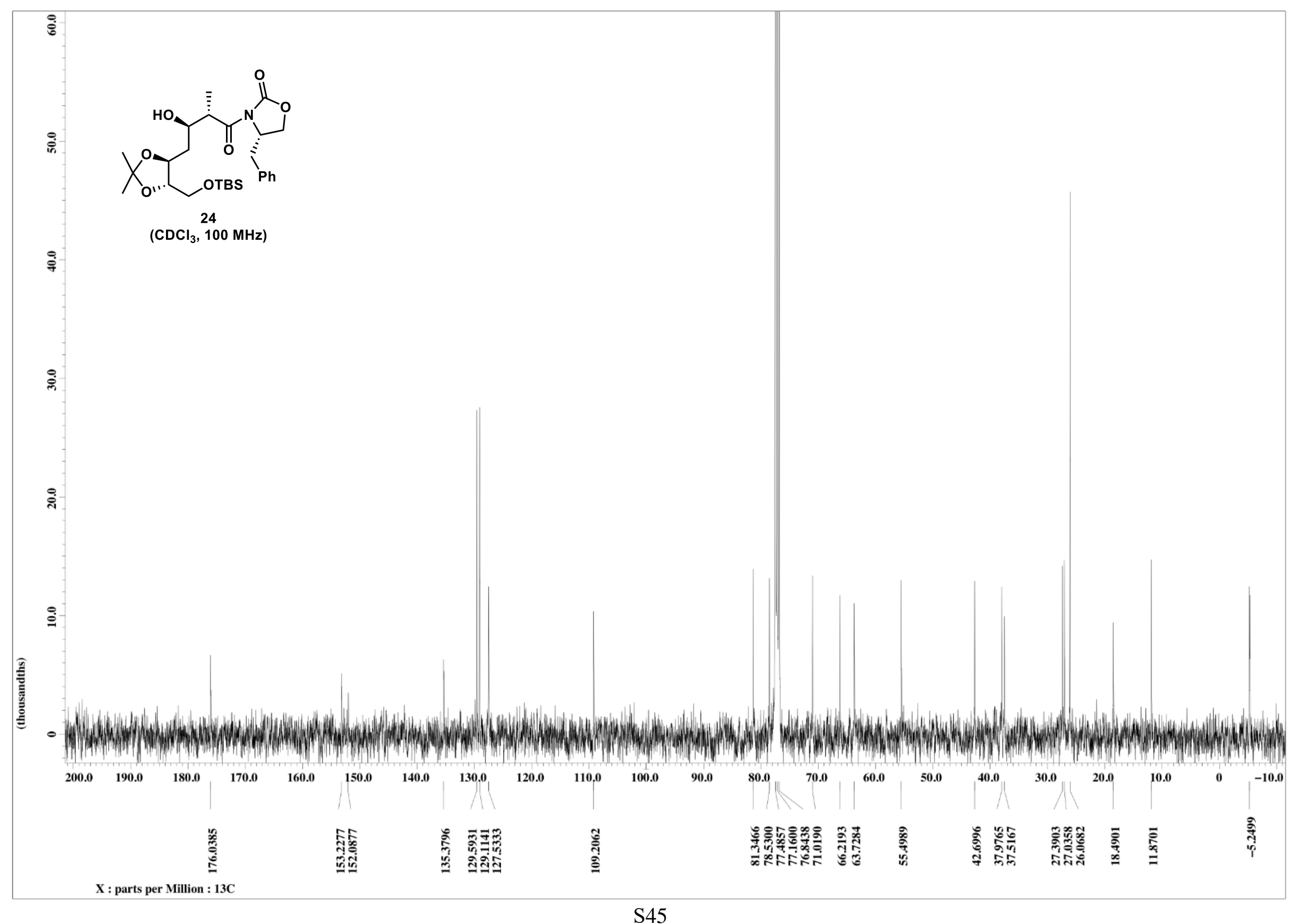




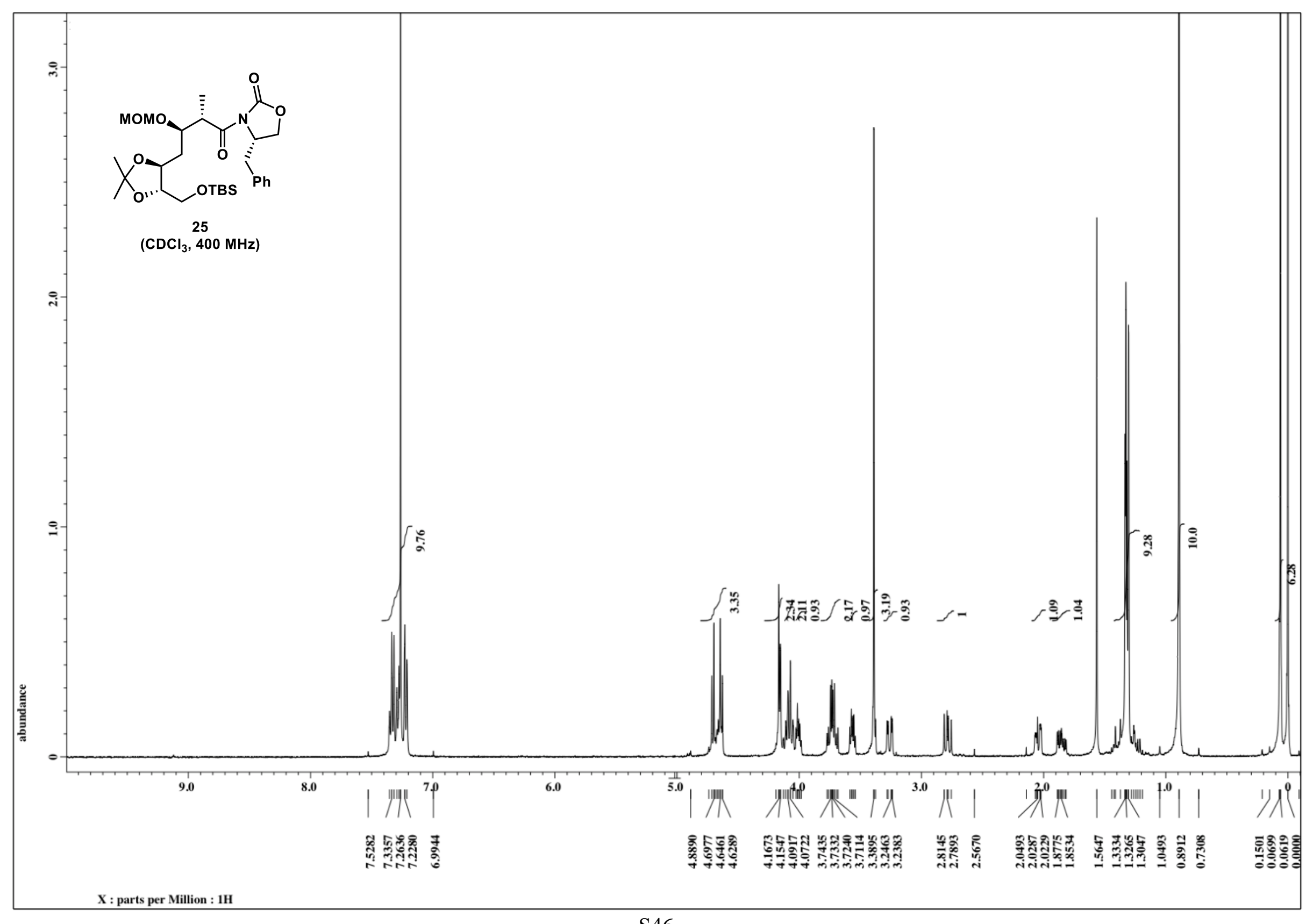




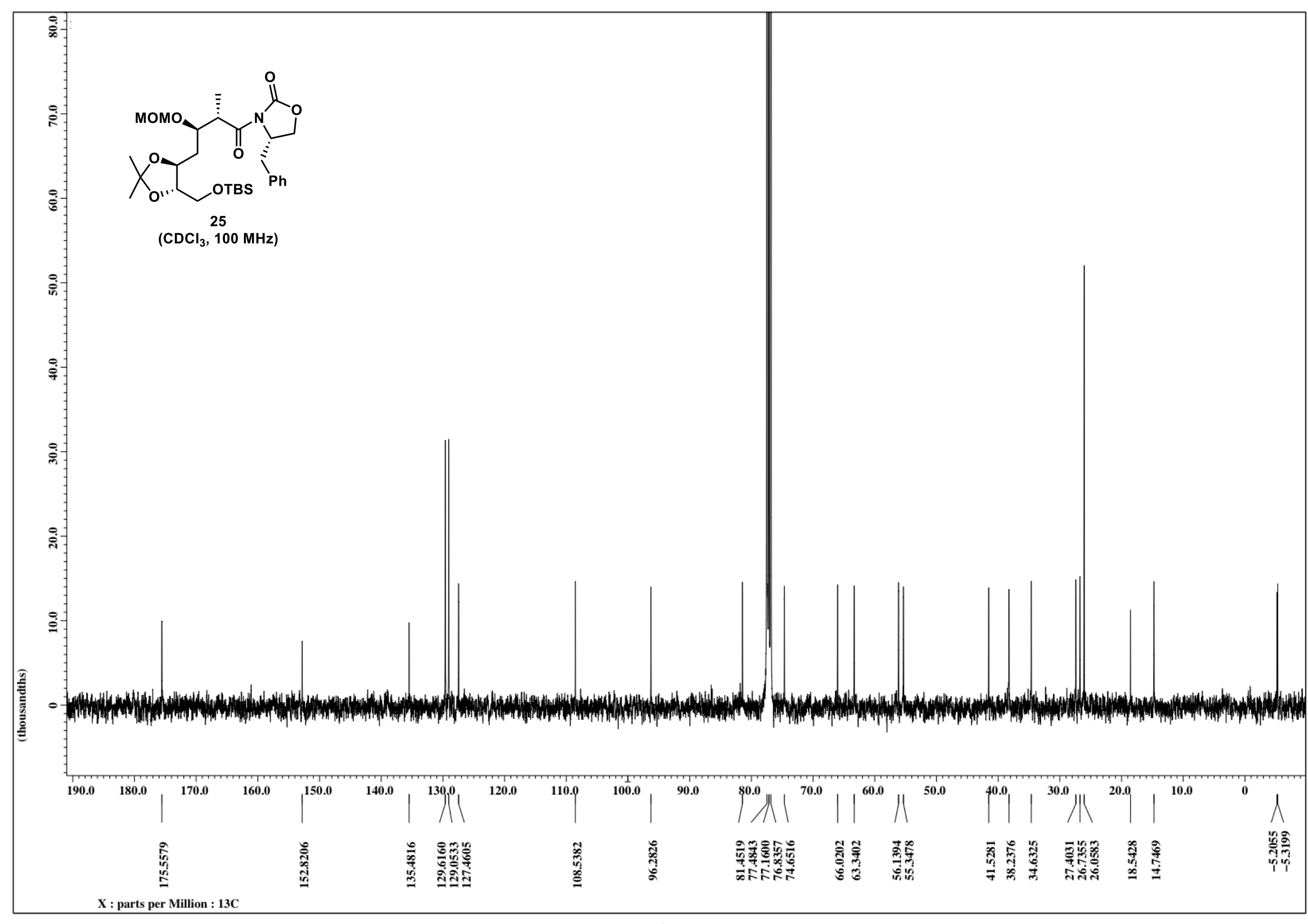




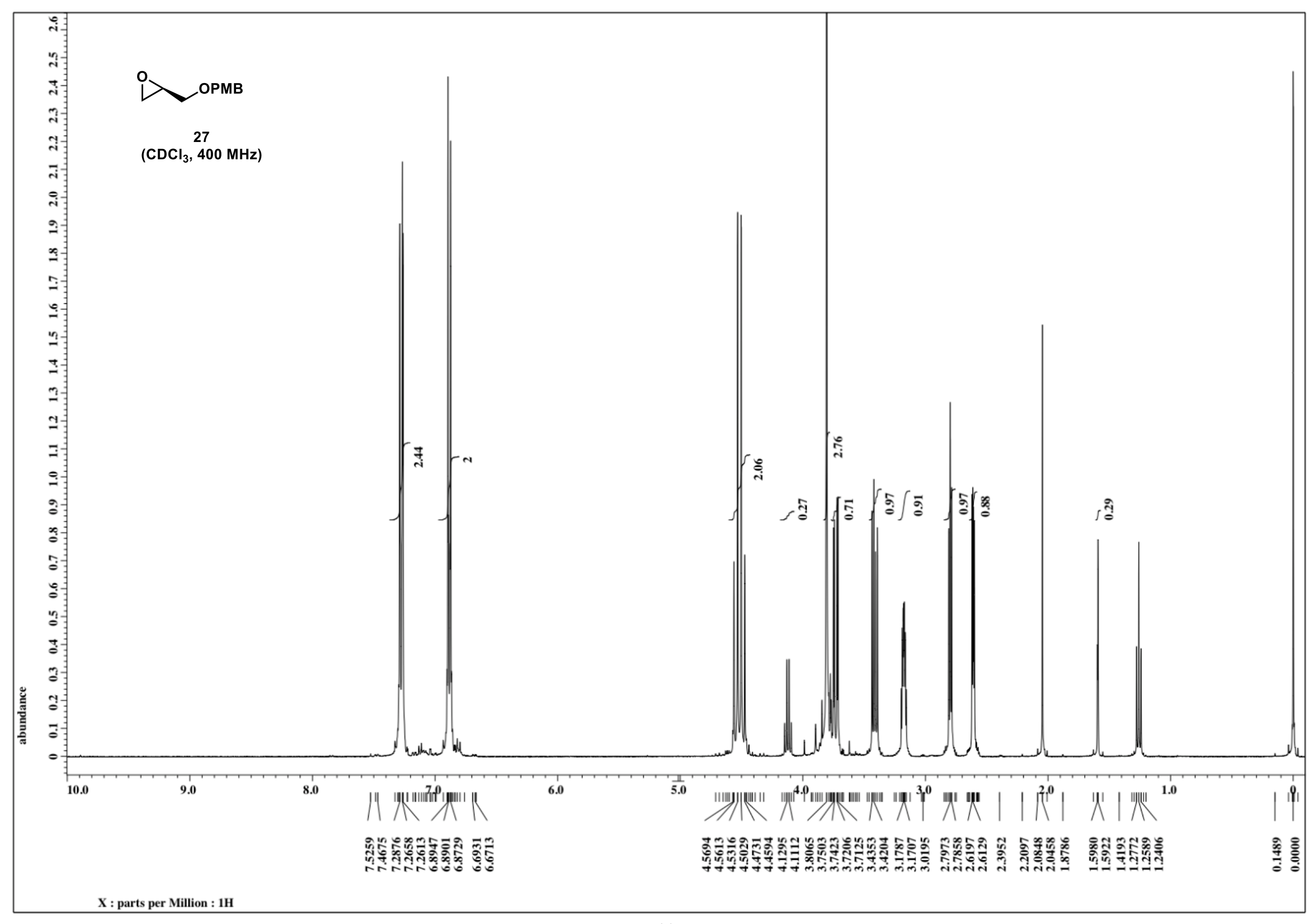




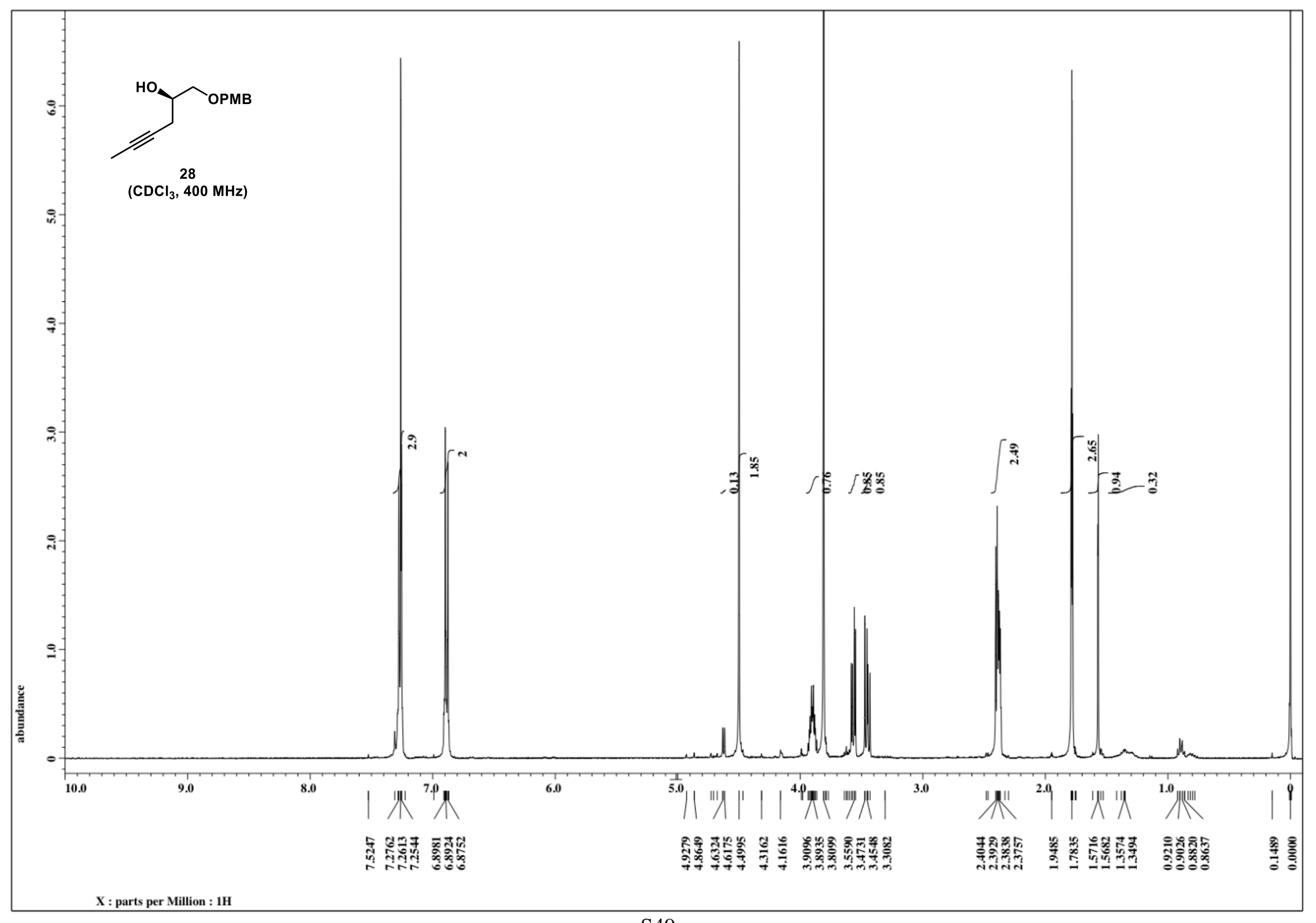




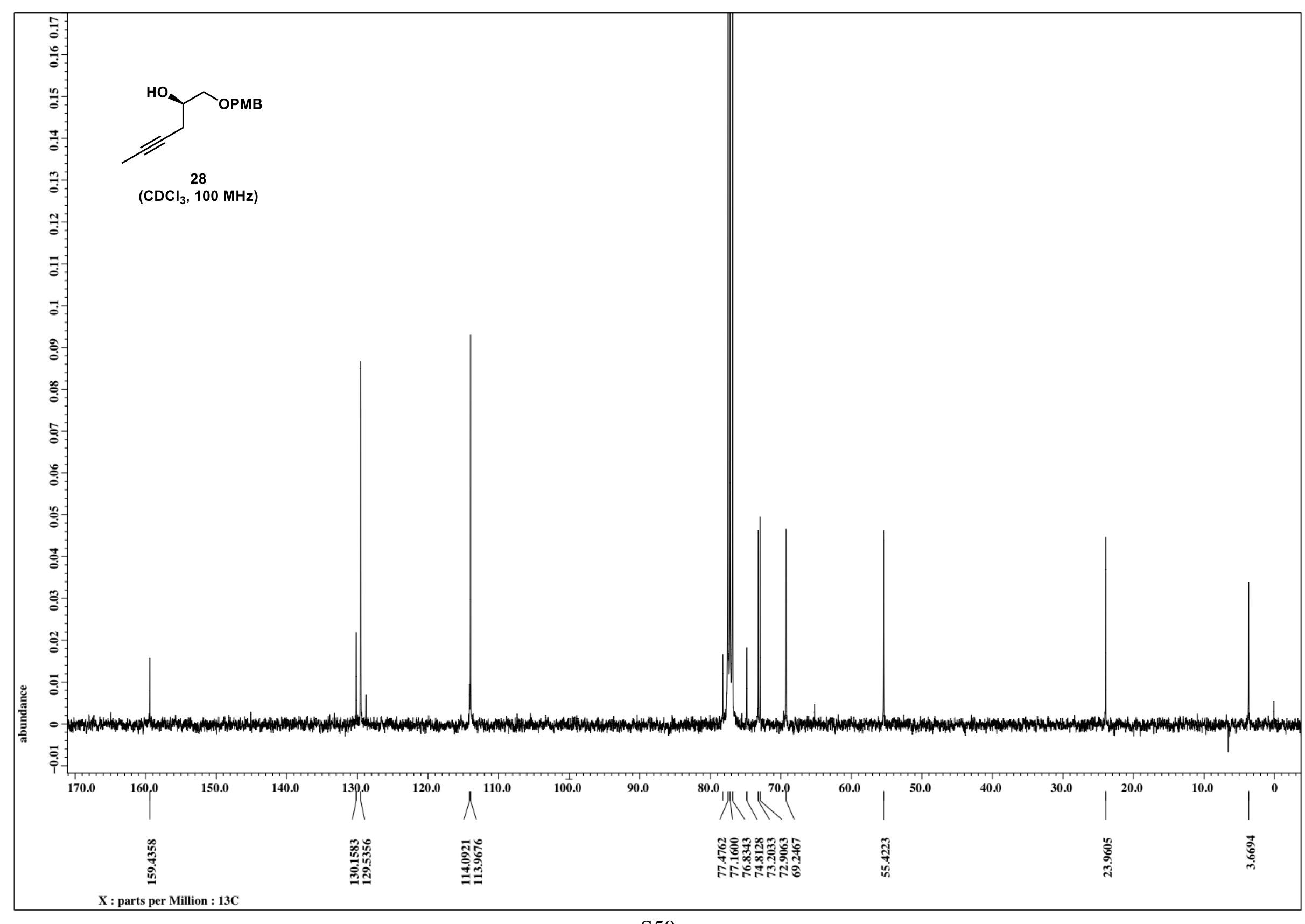




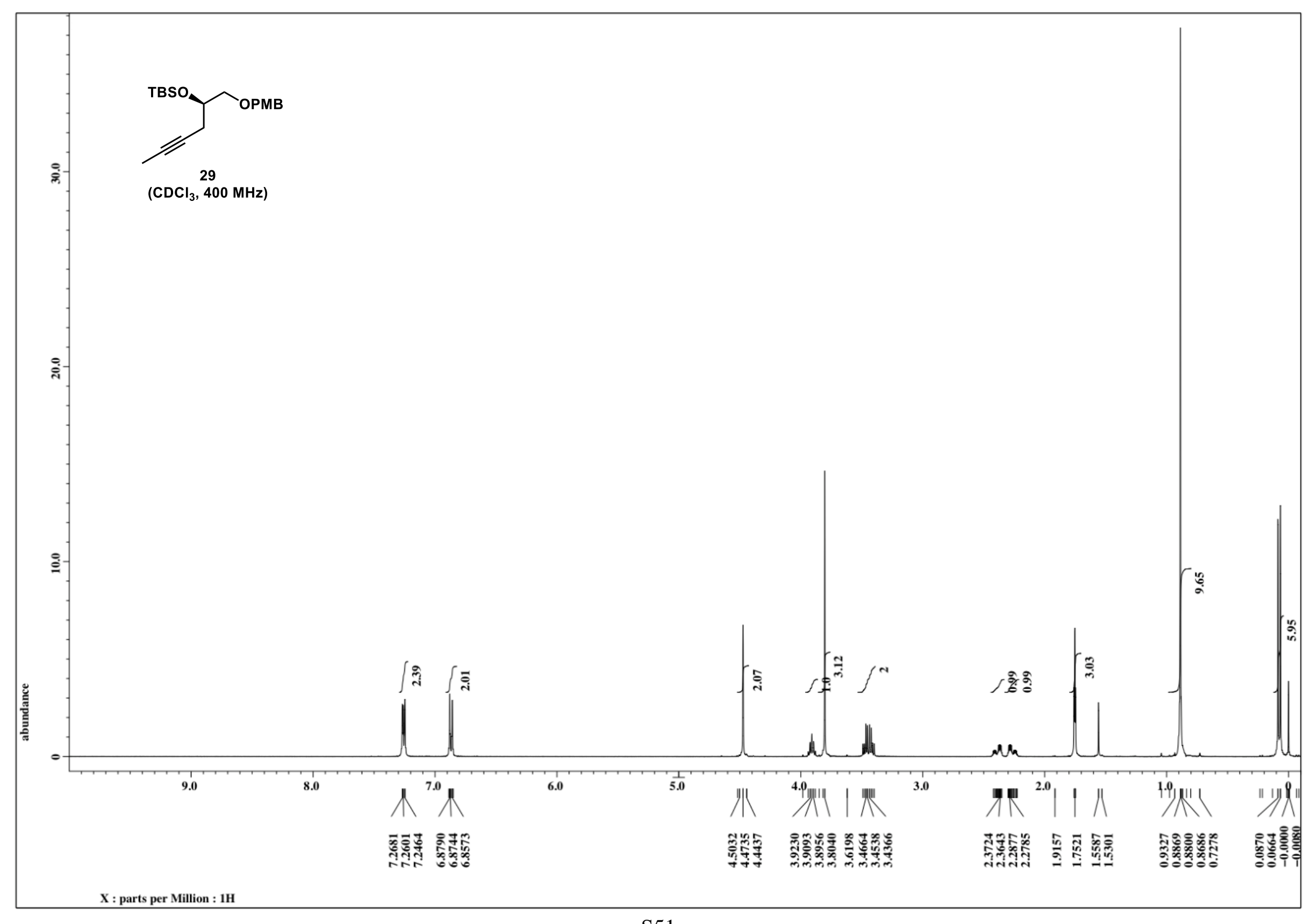




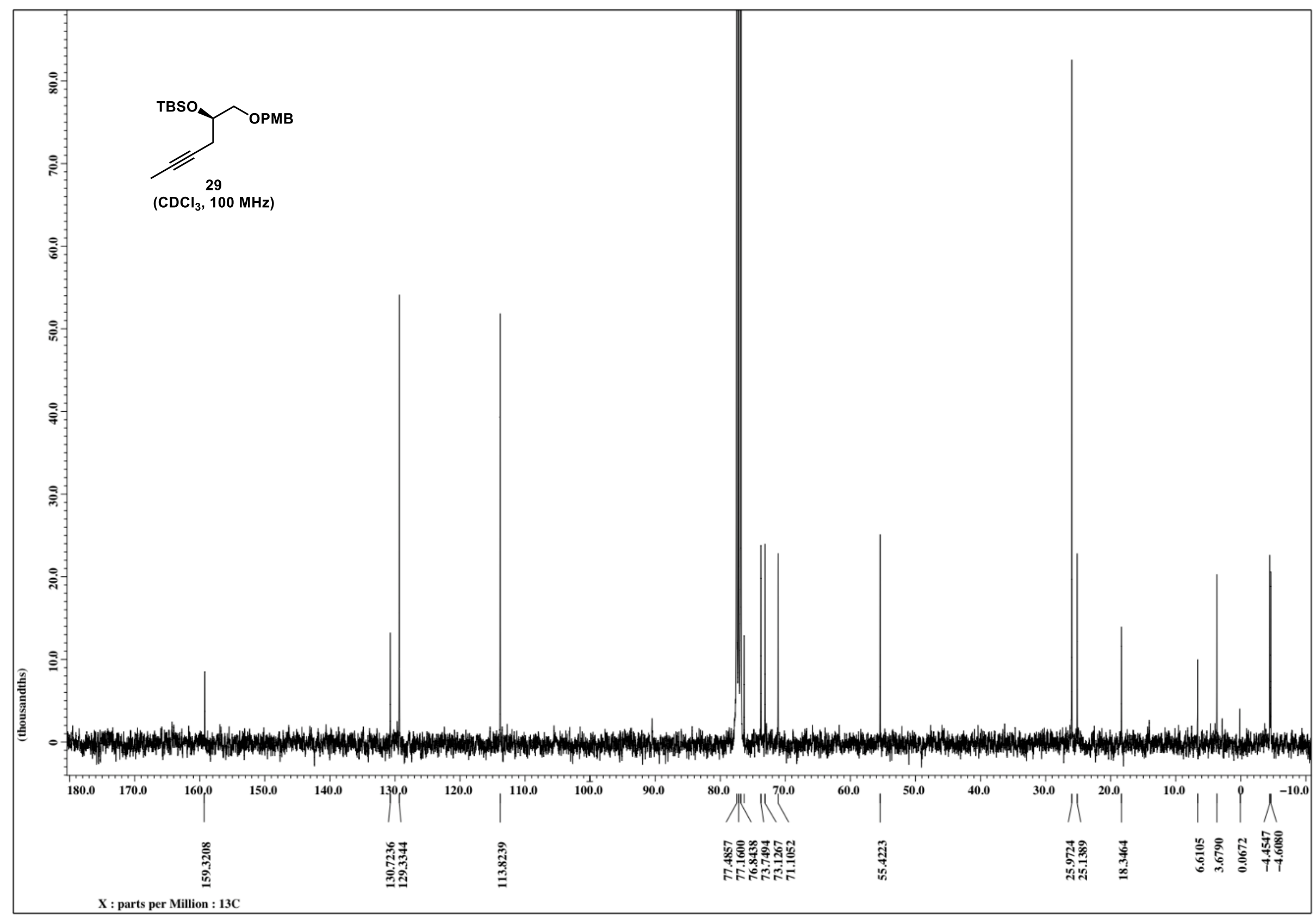




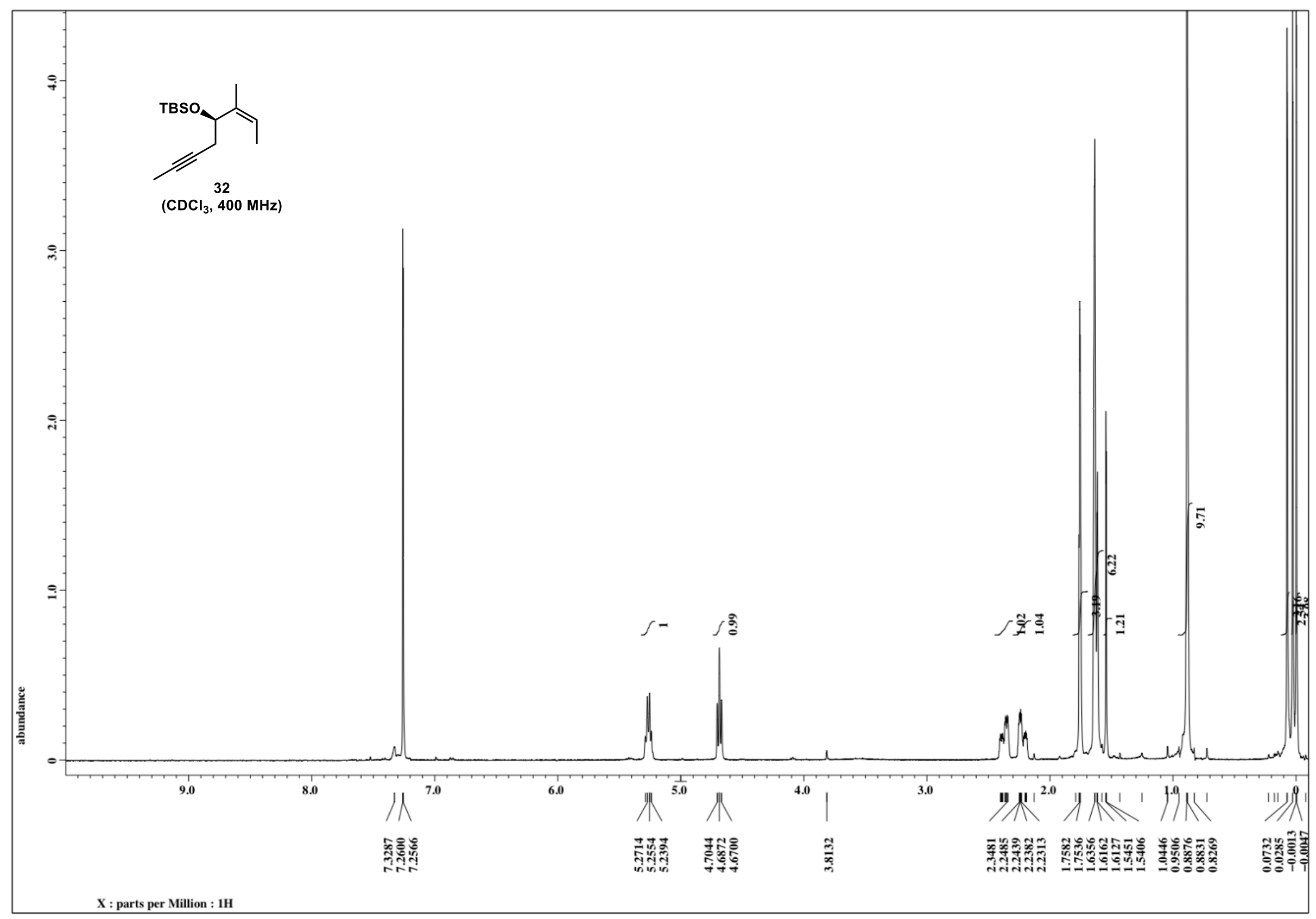




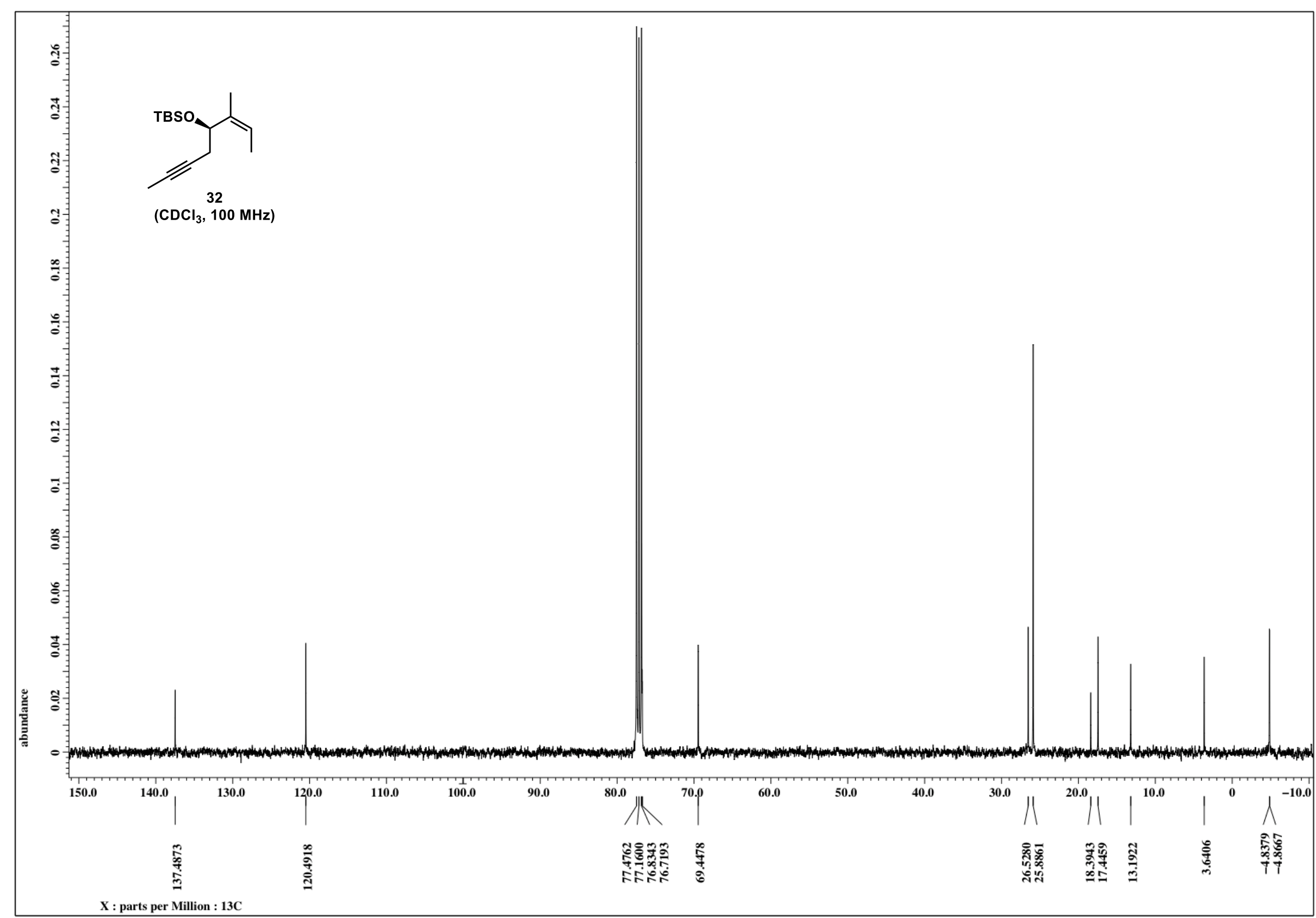




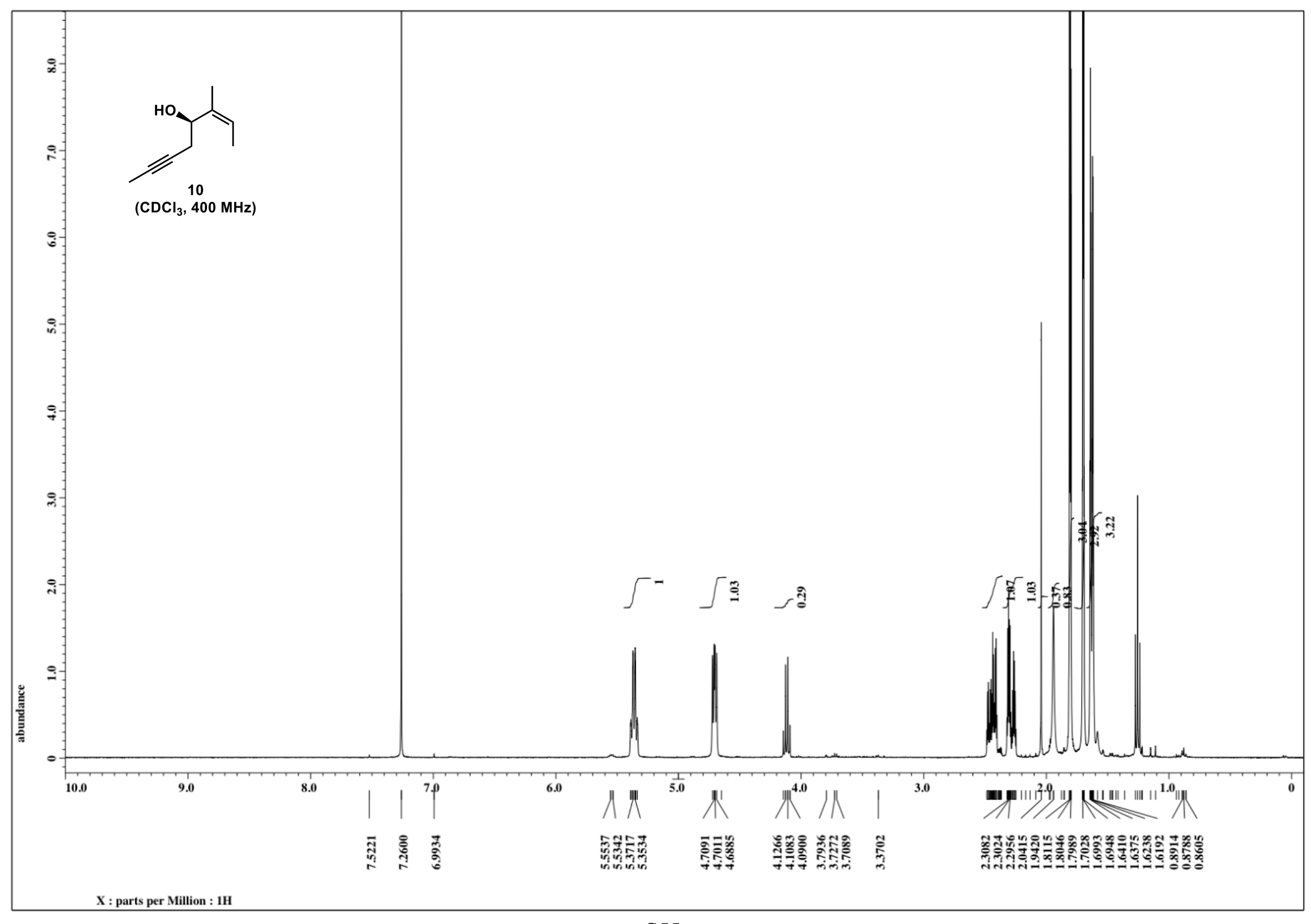




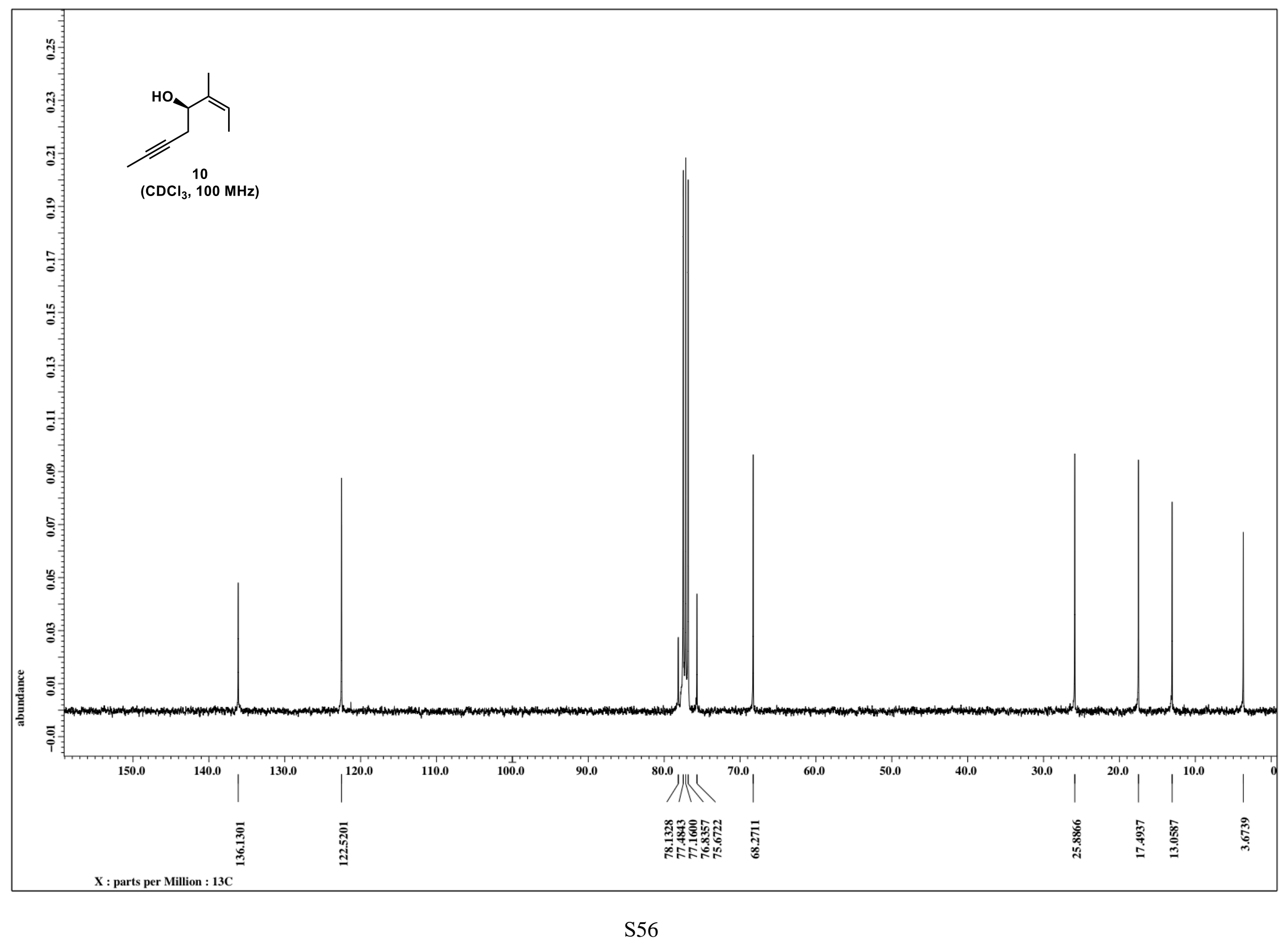




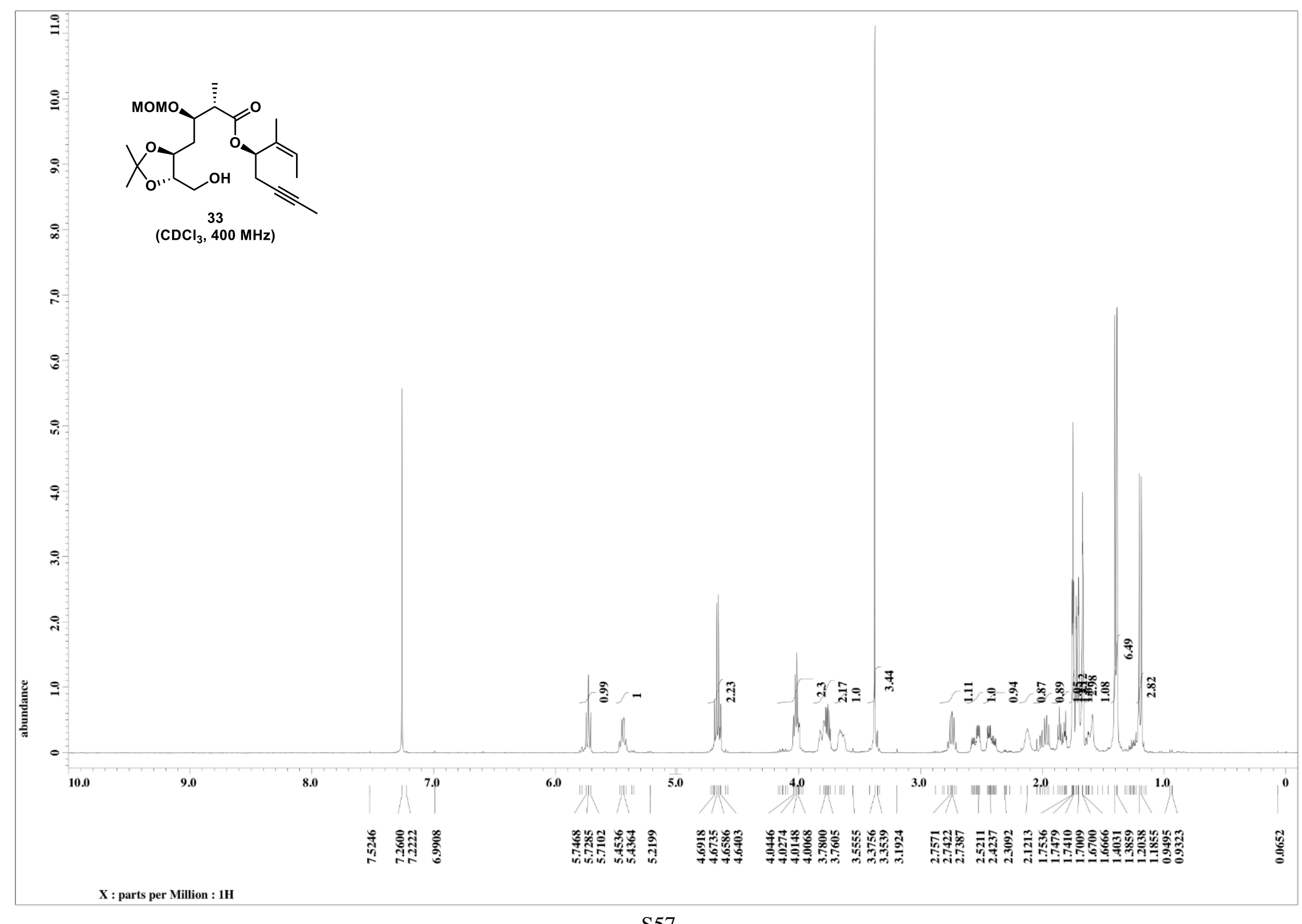




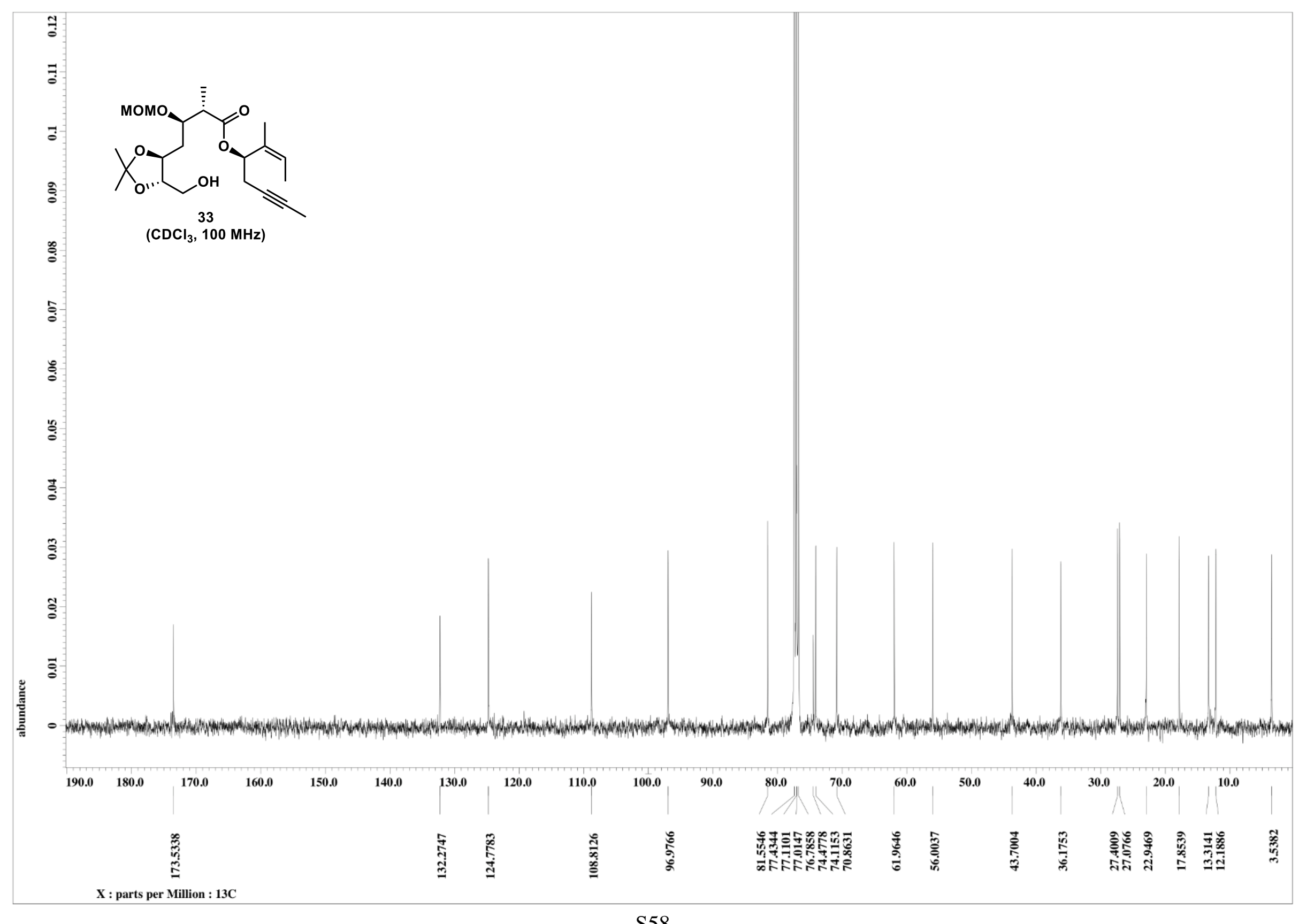




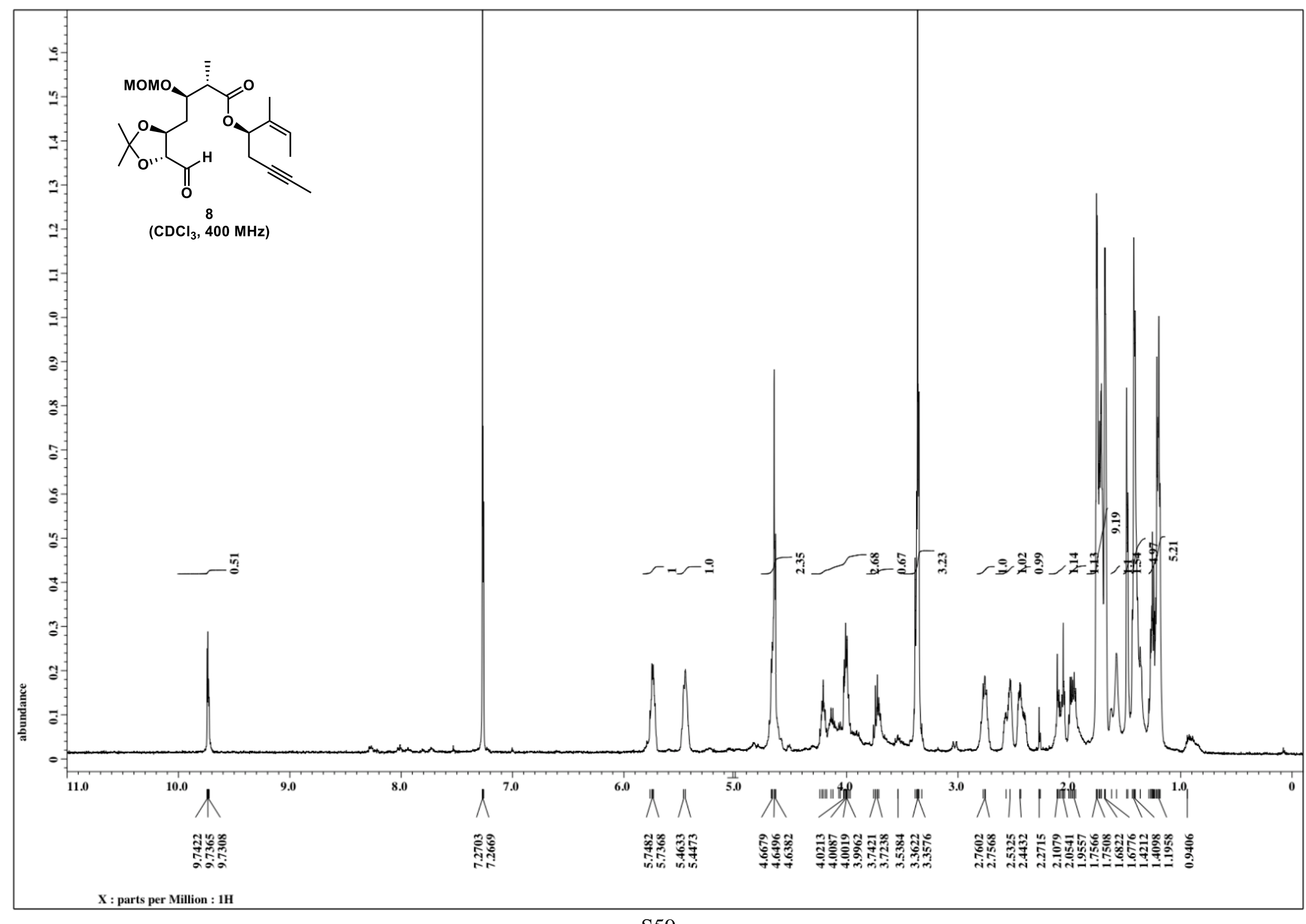




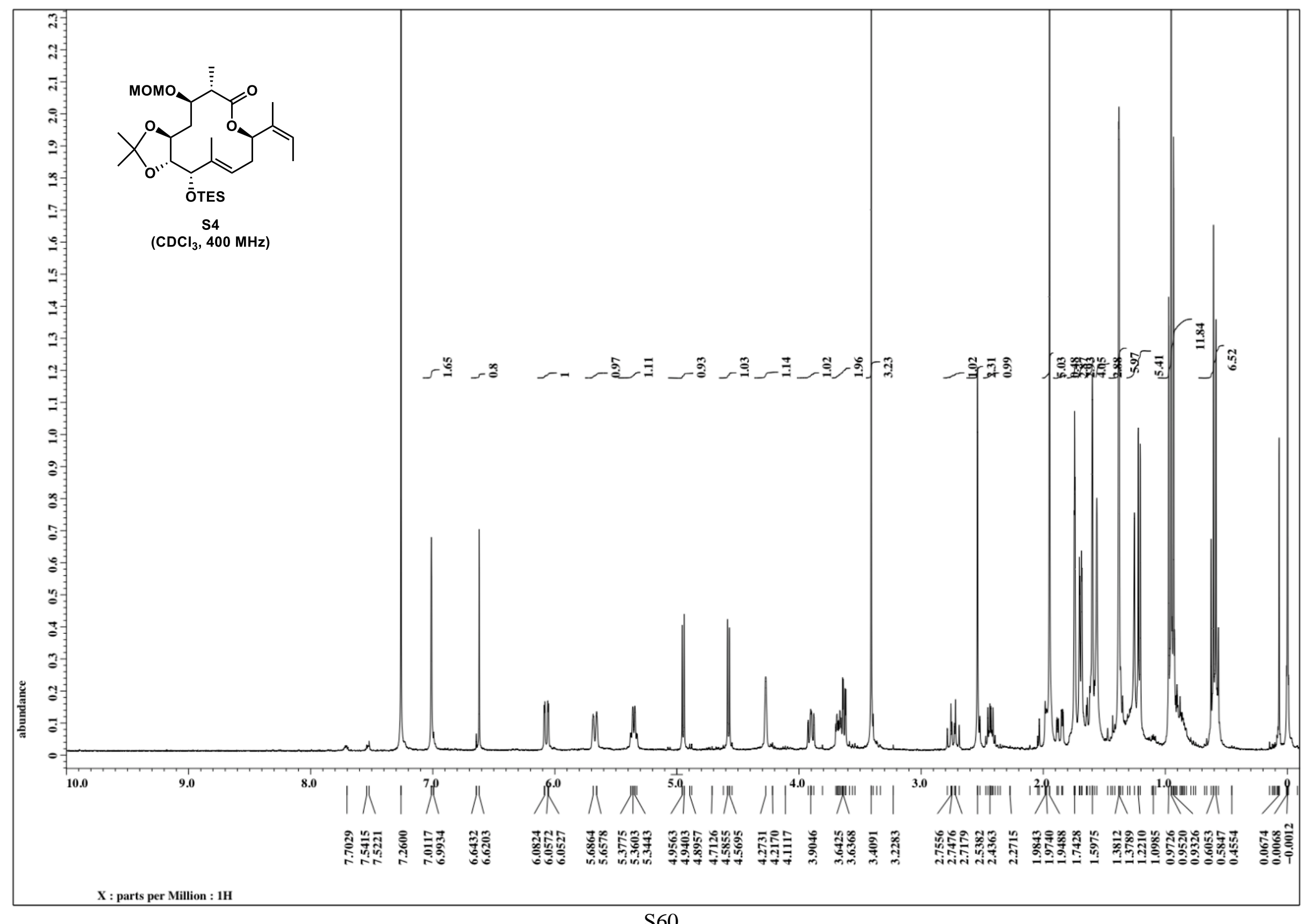




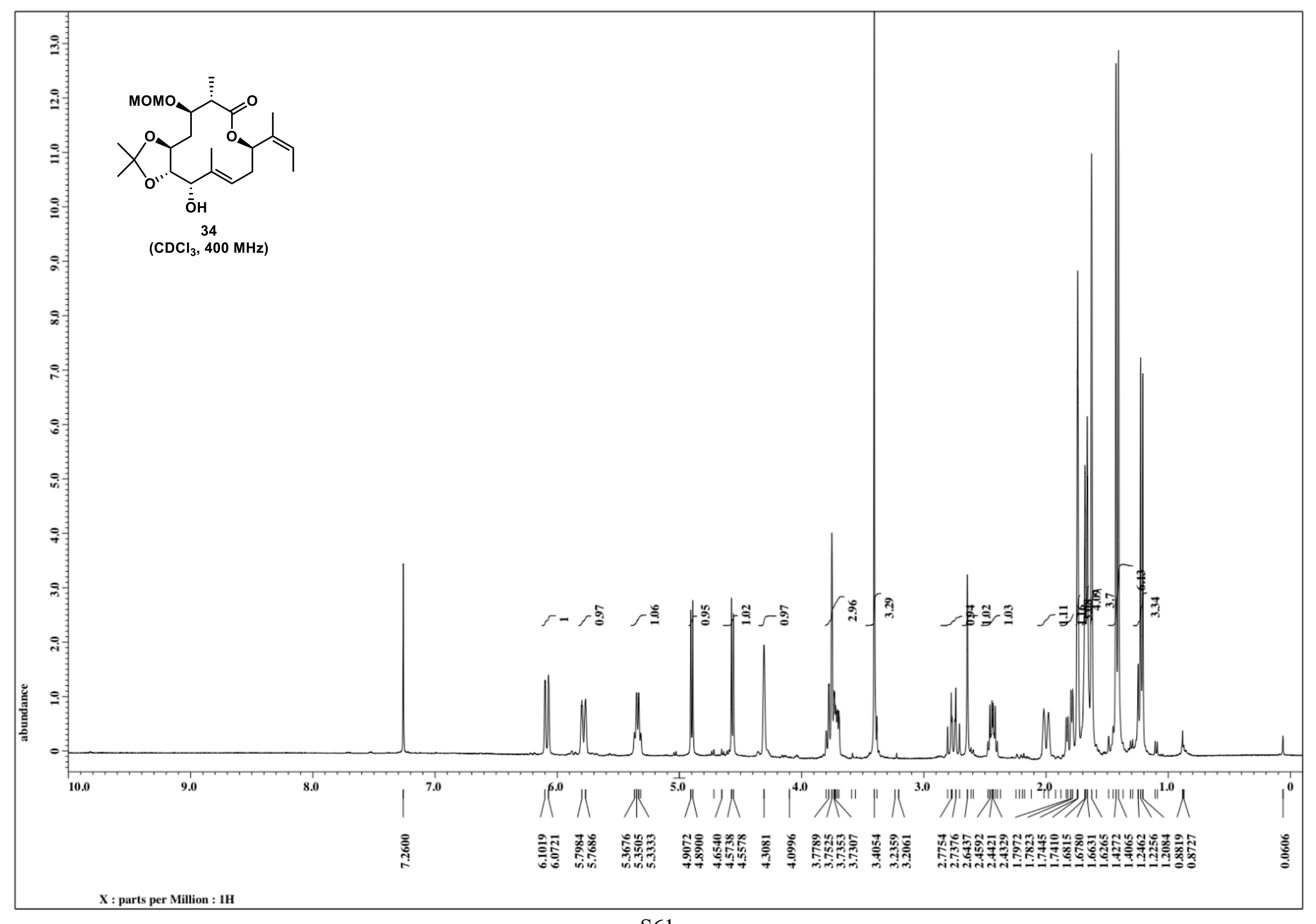




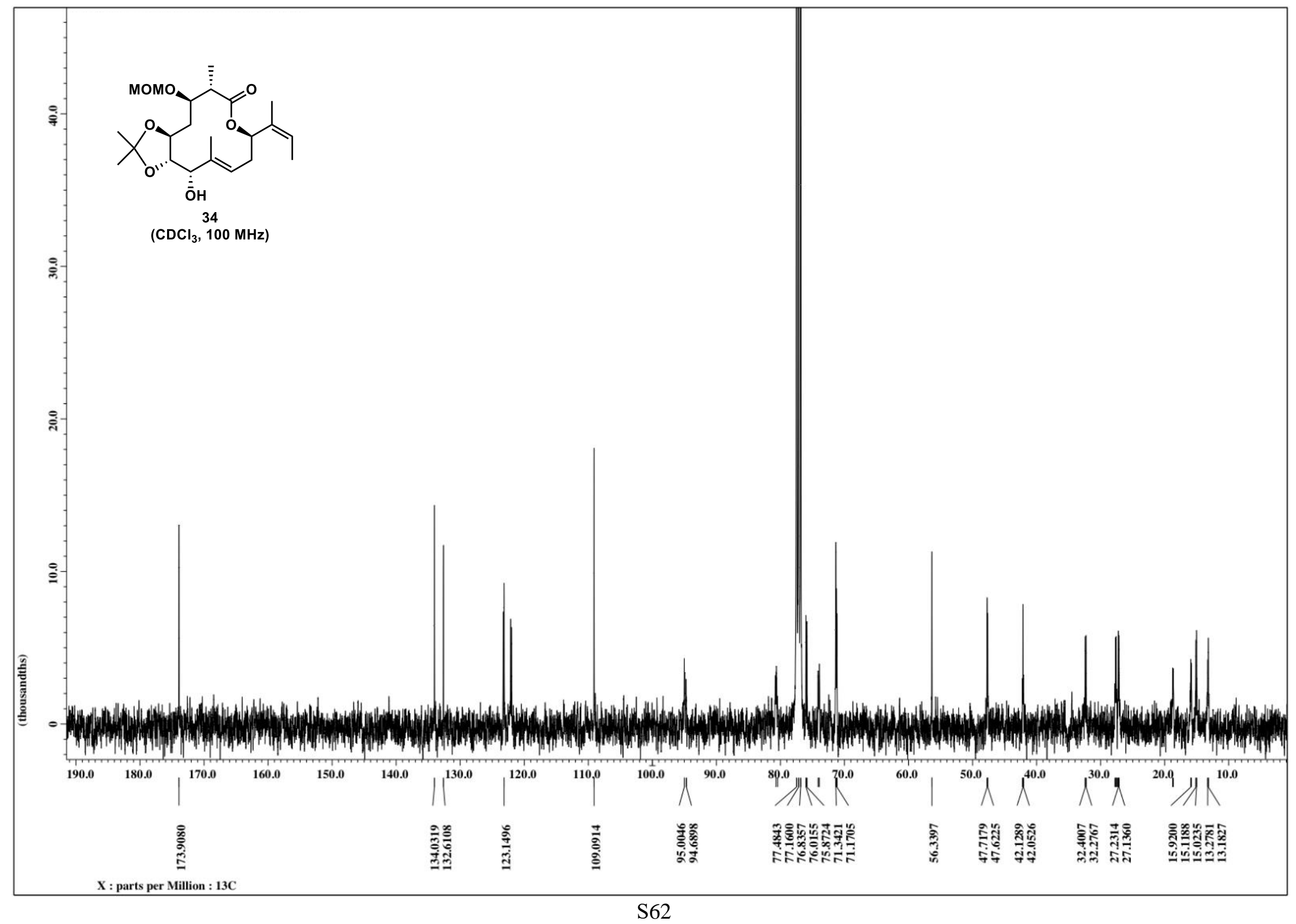




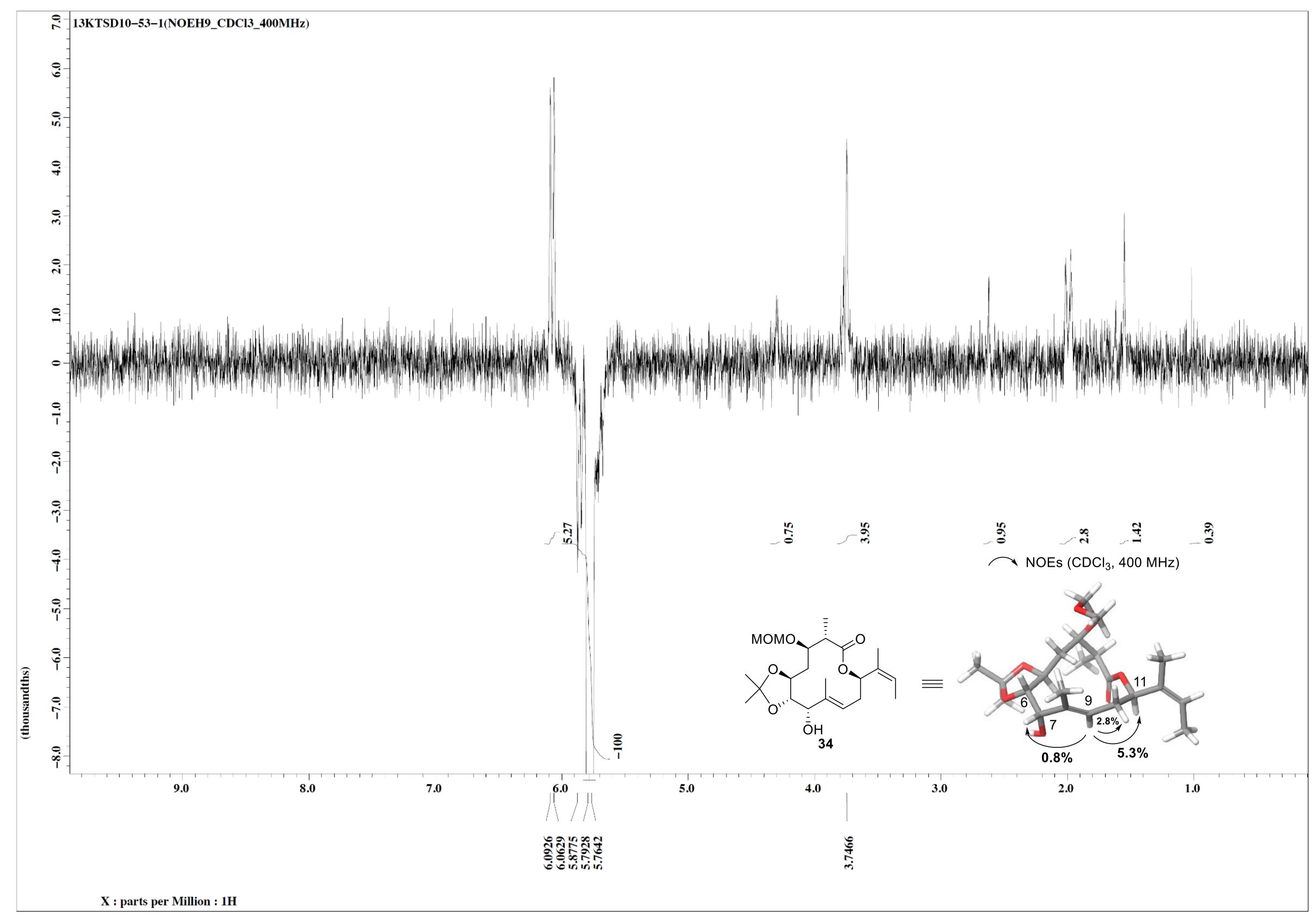




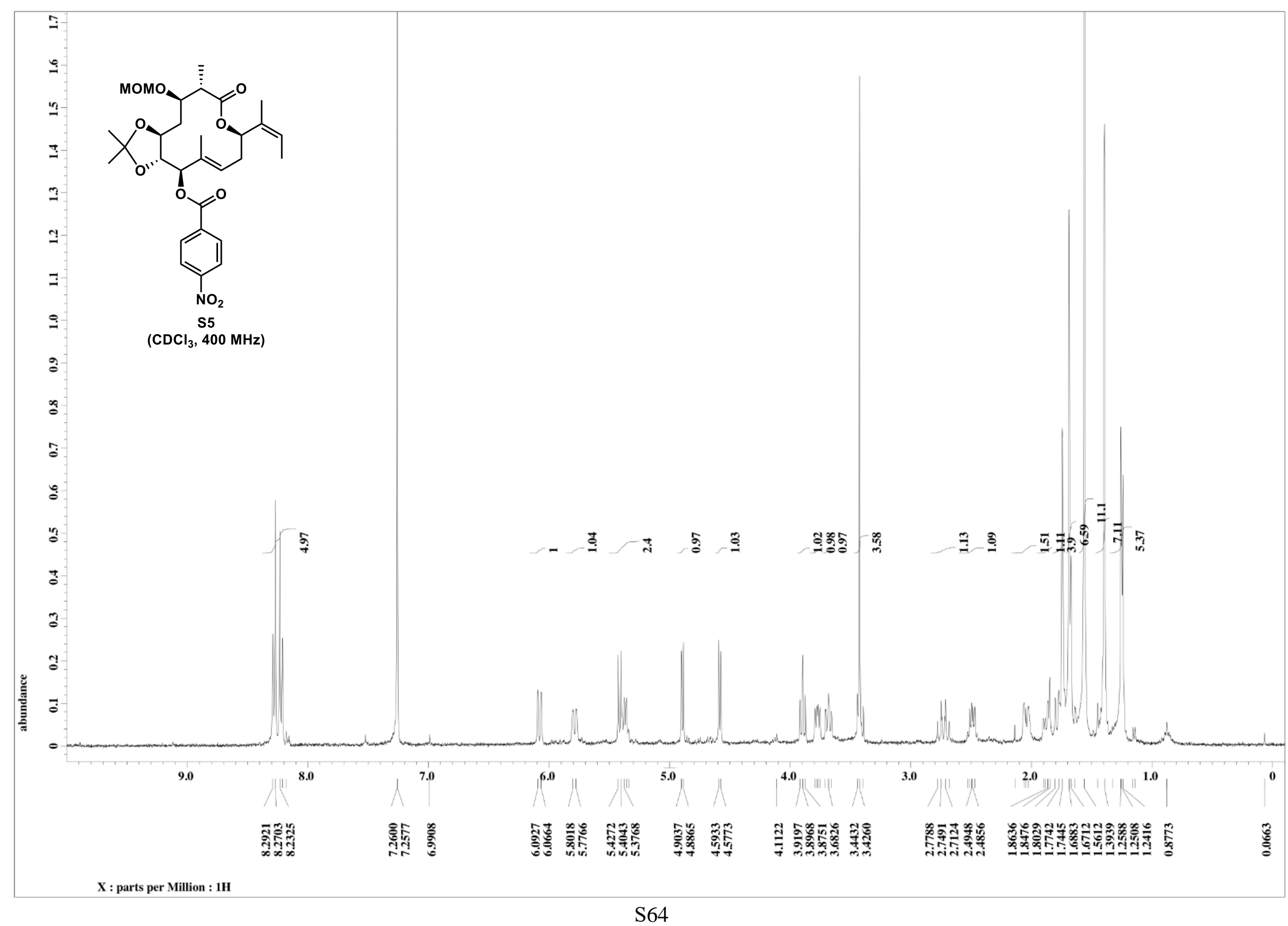




$$
1
$$




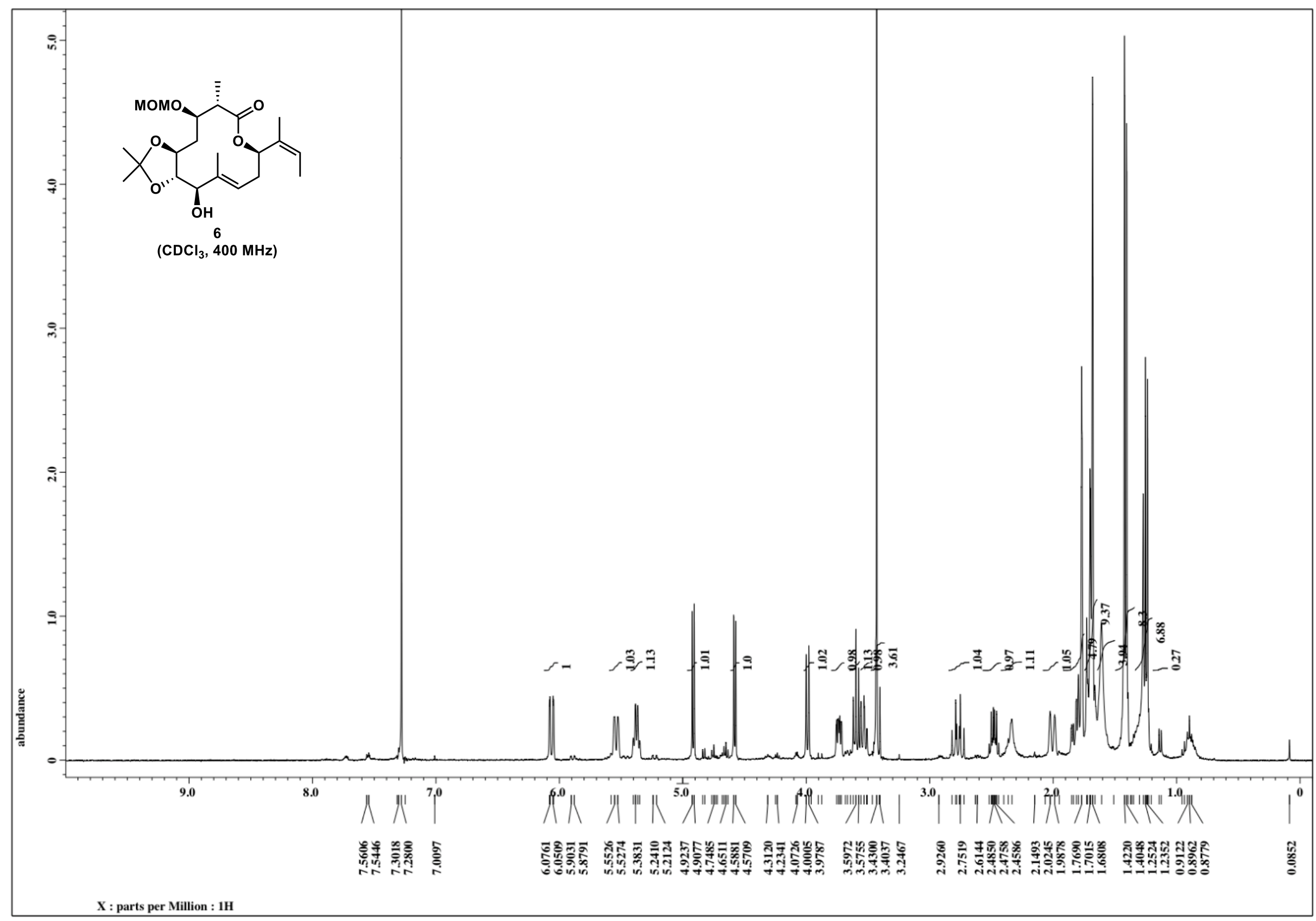




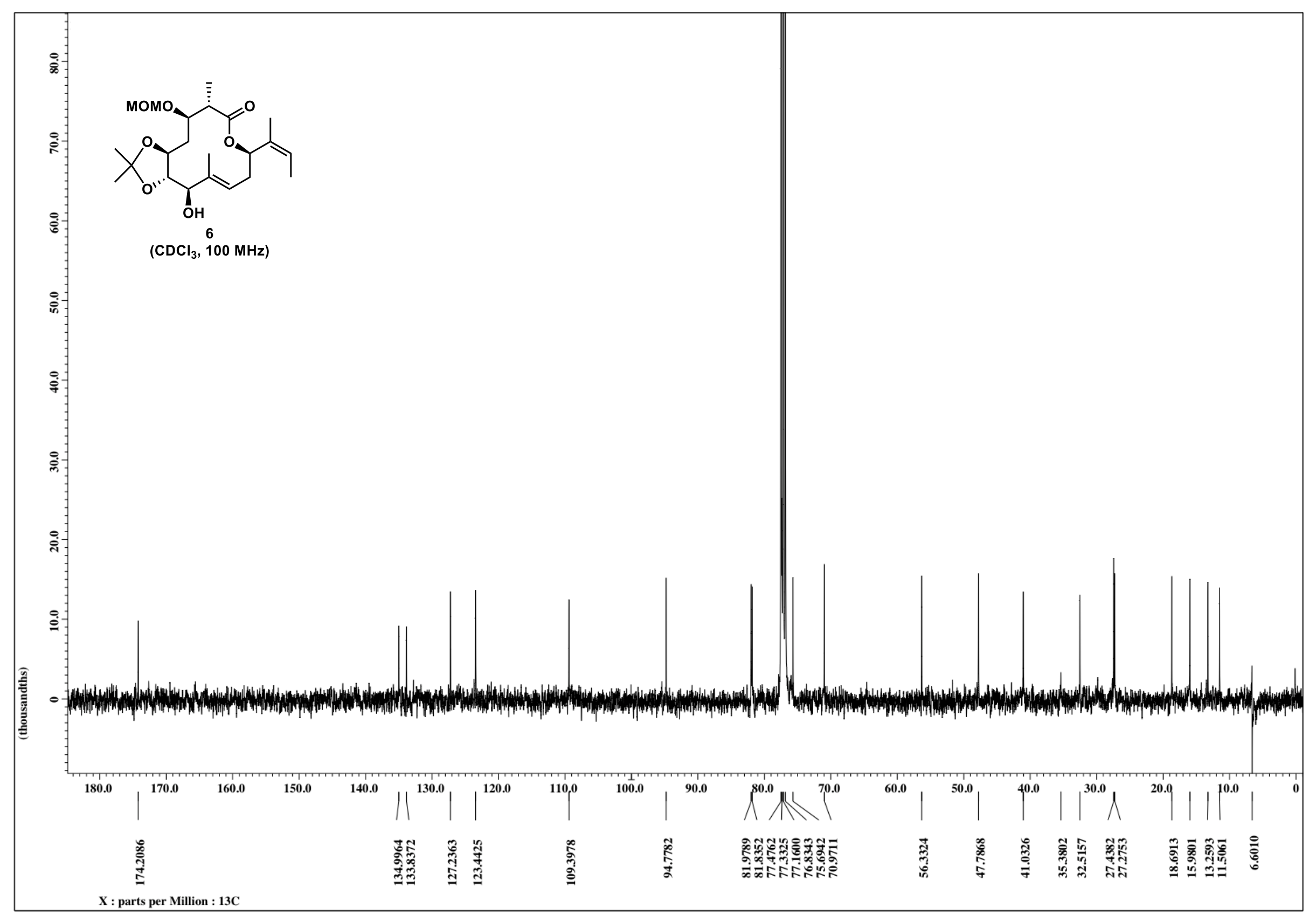




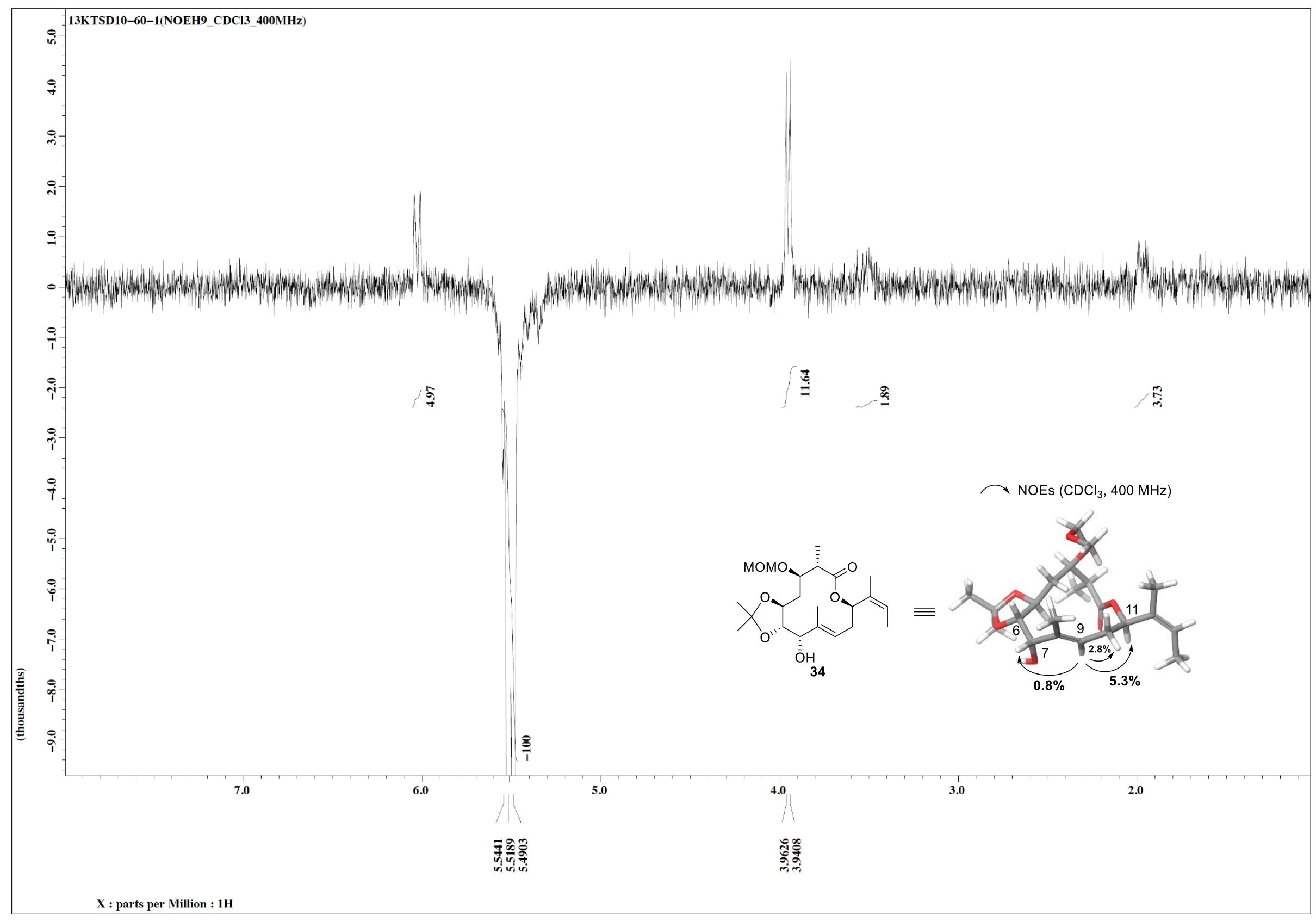




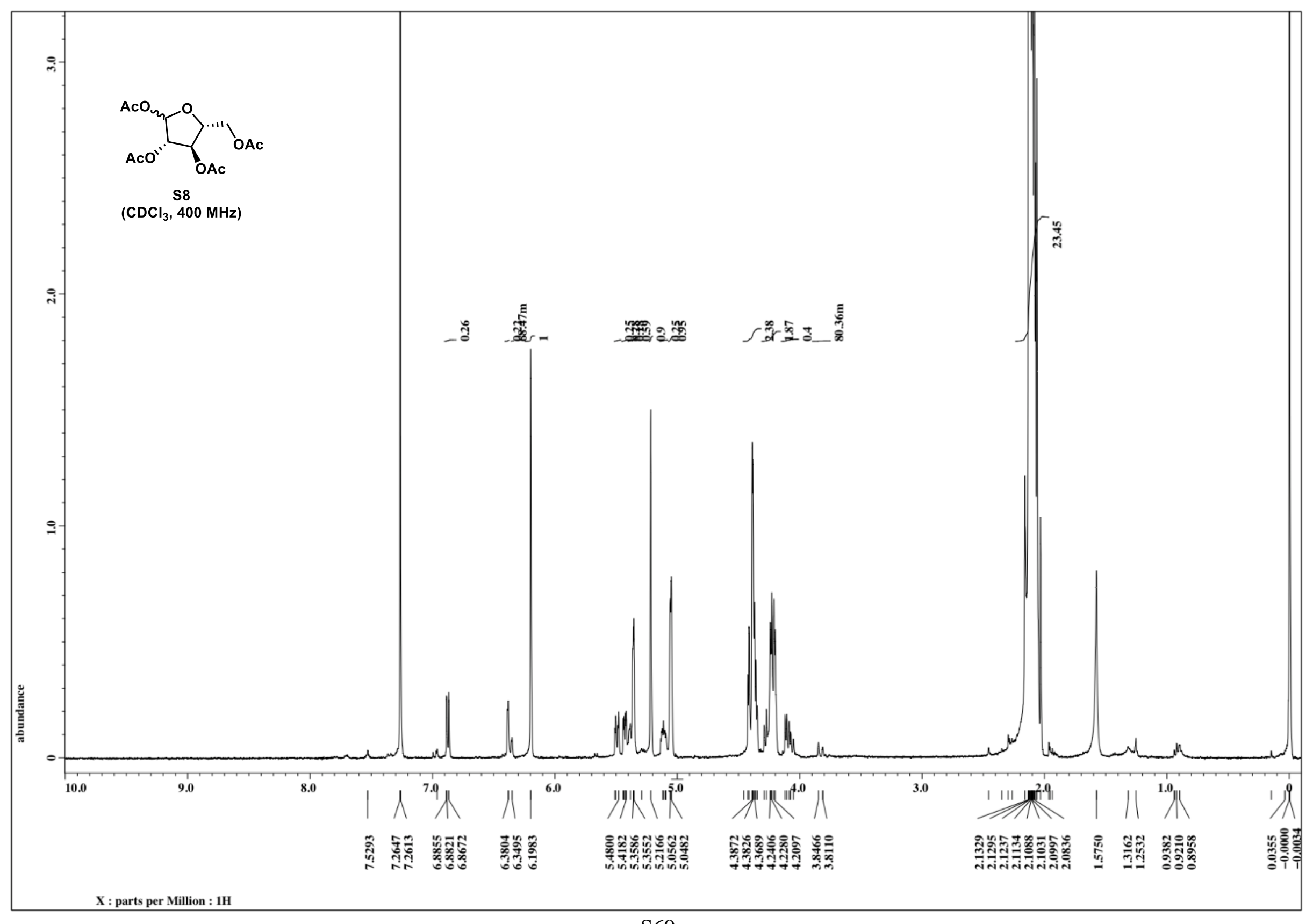




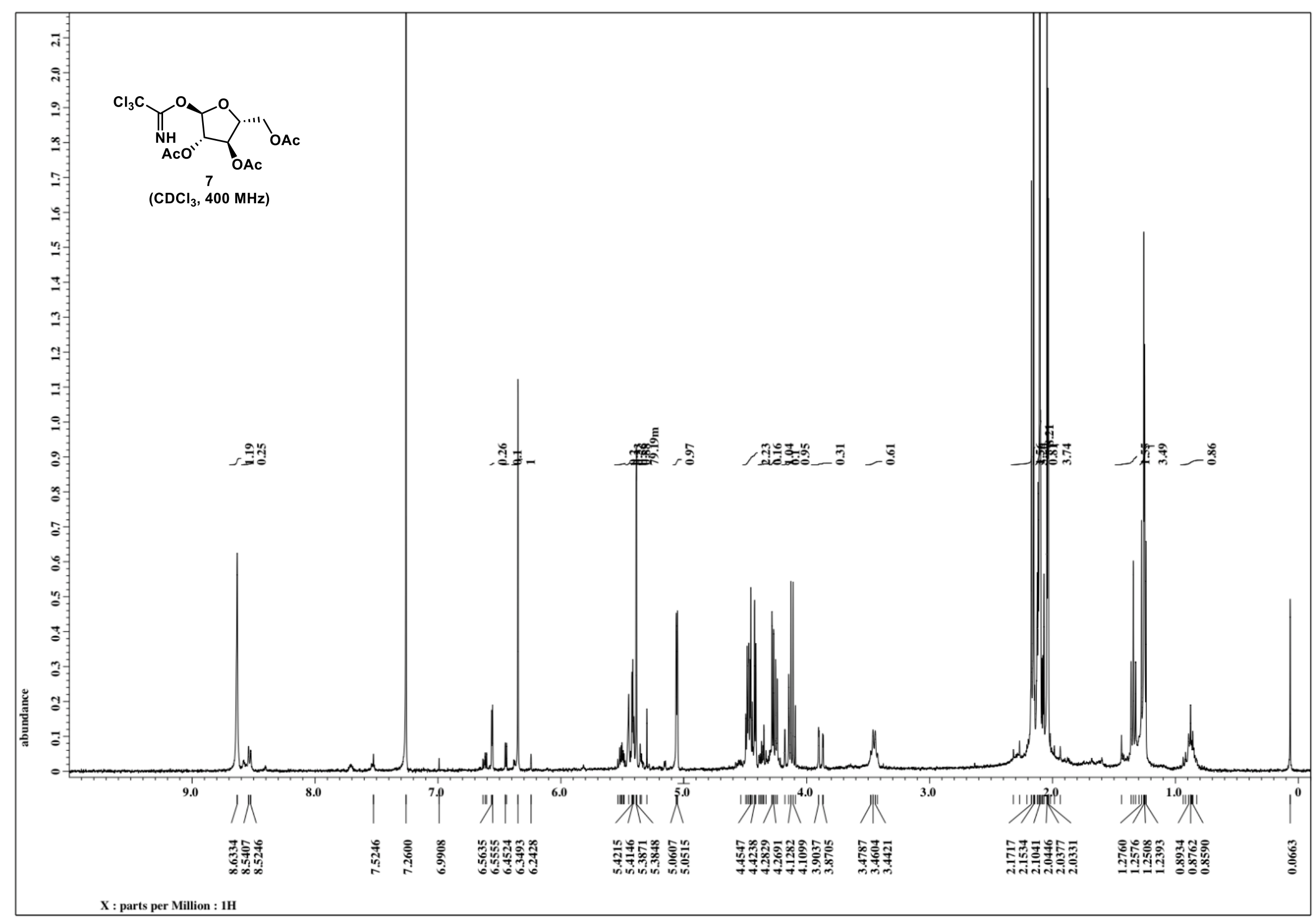




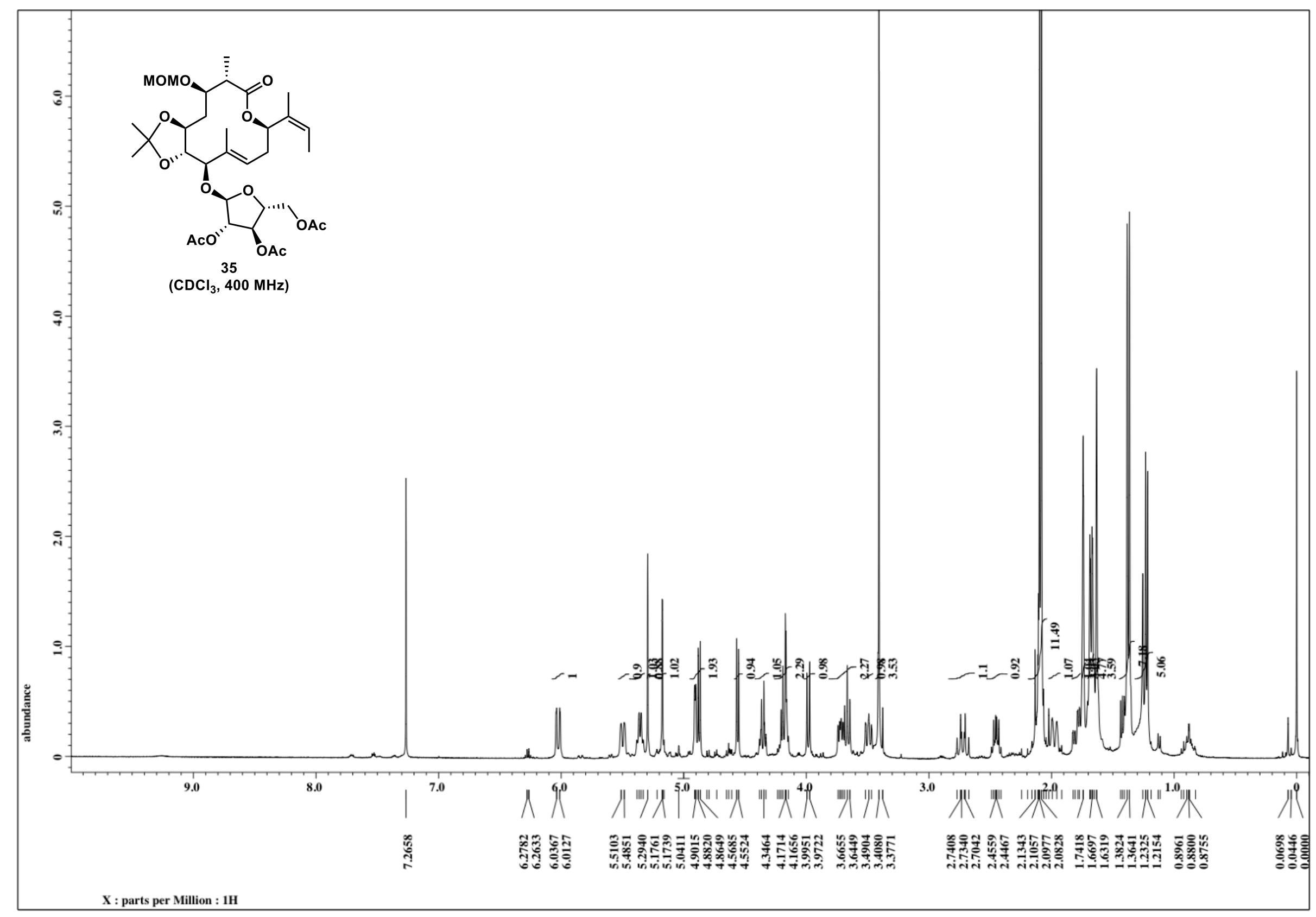




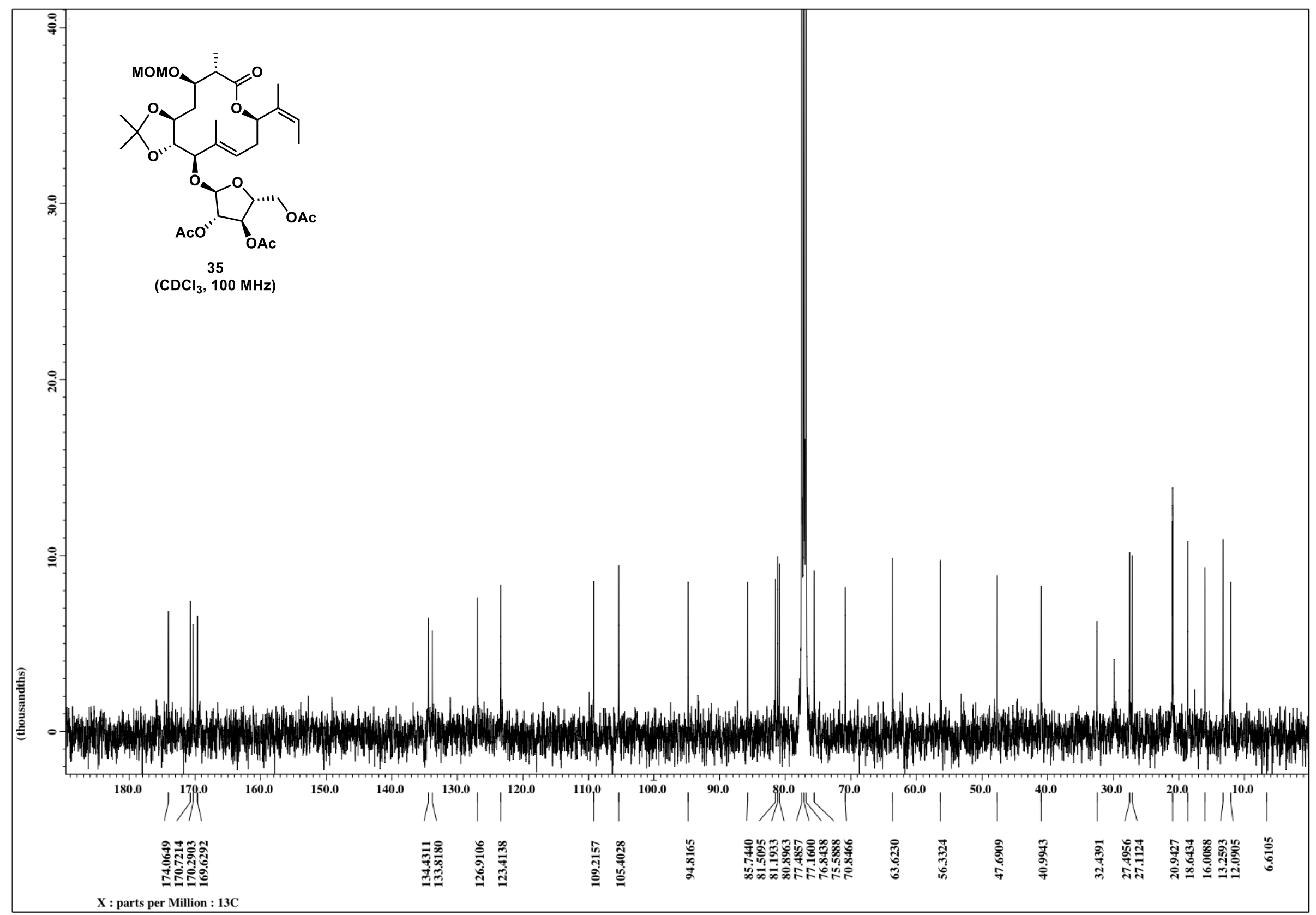




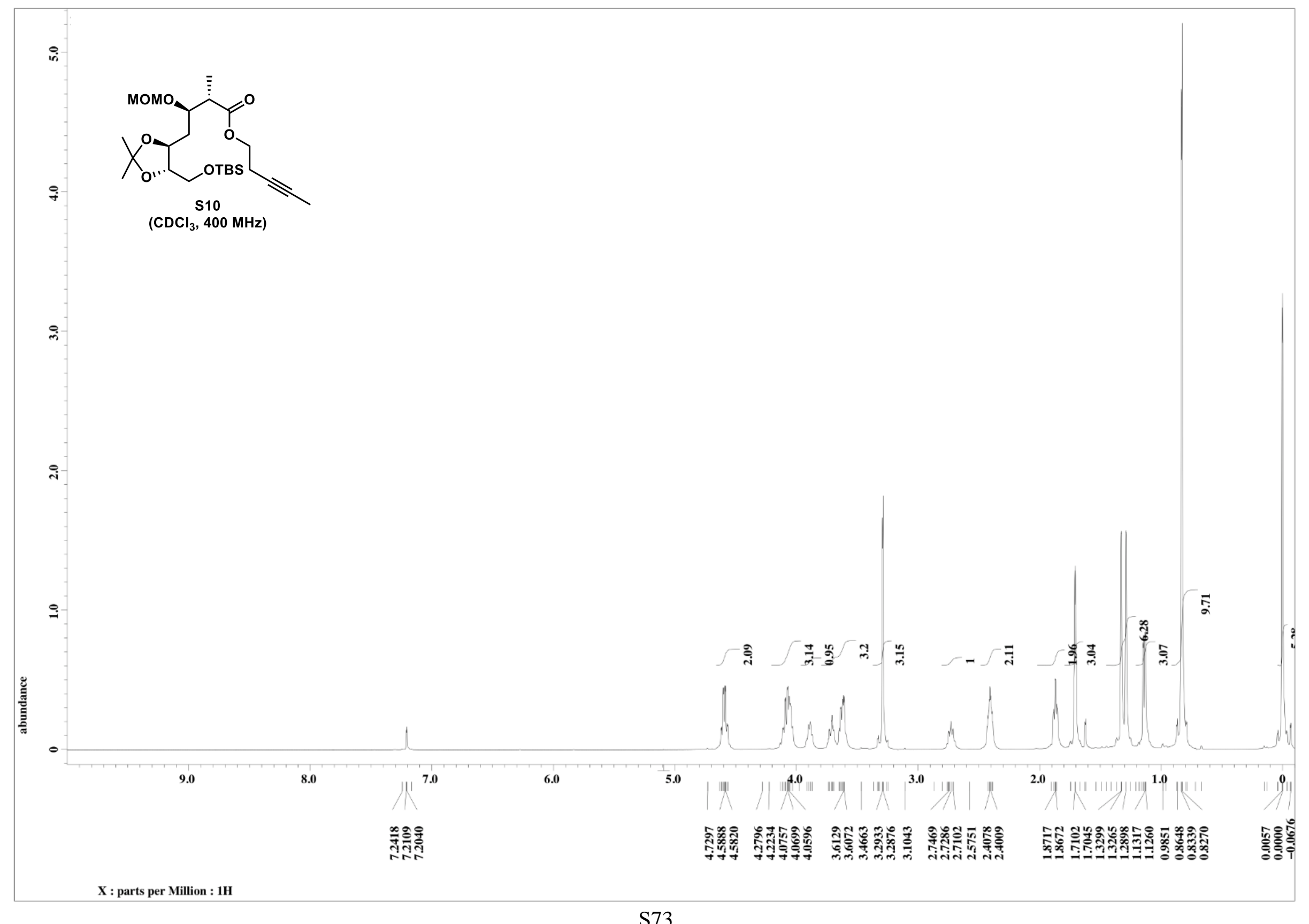




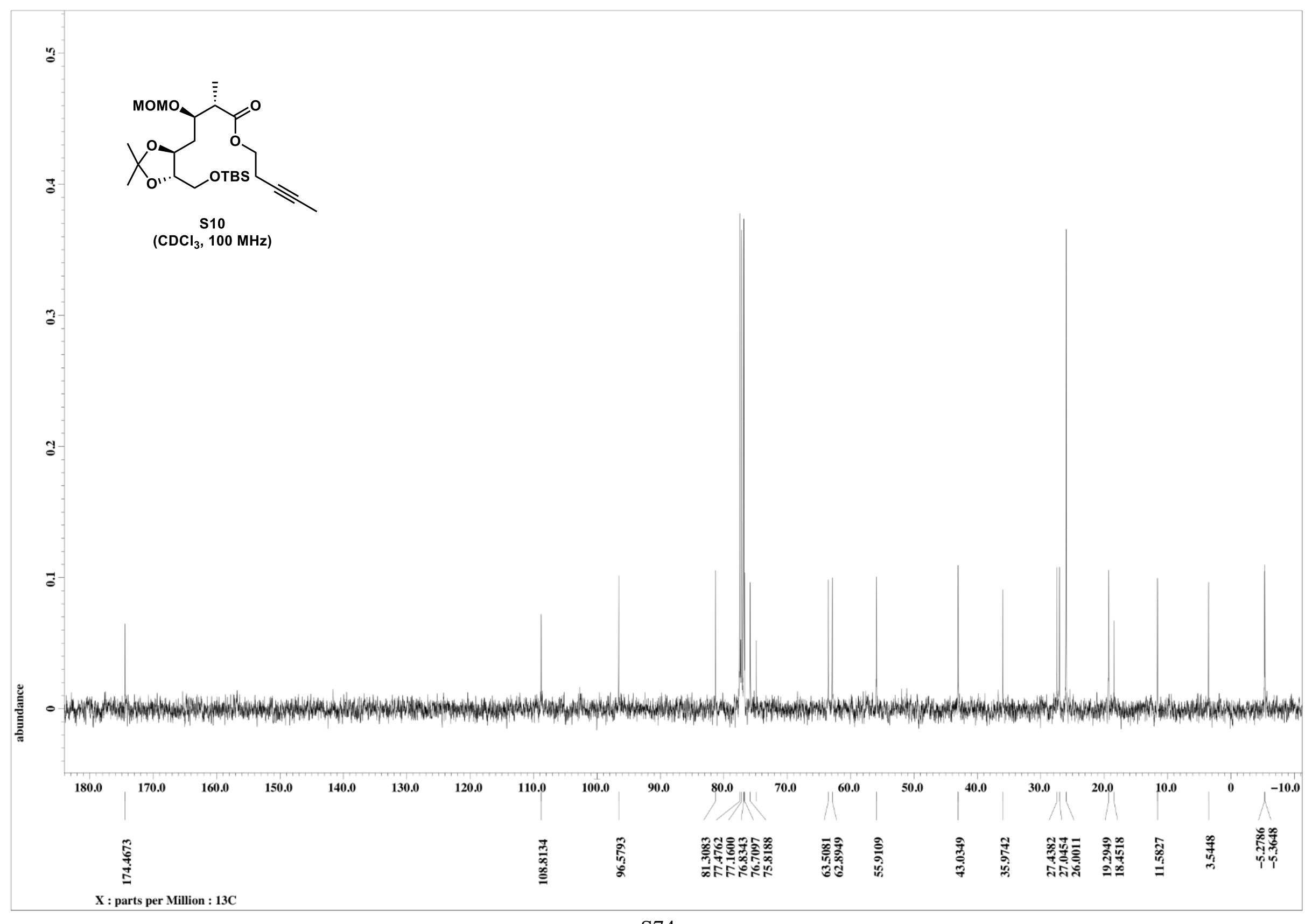




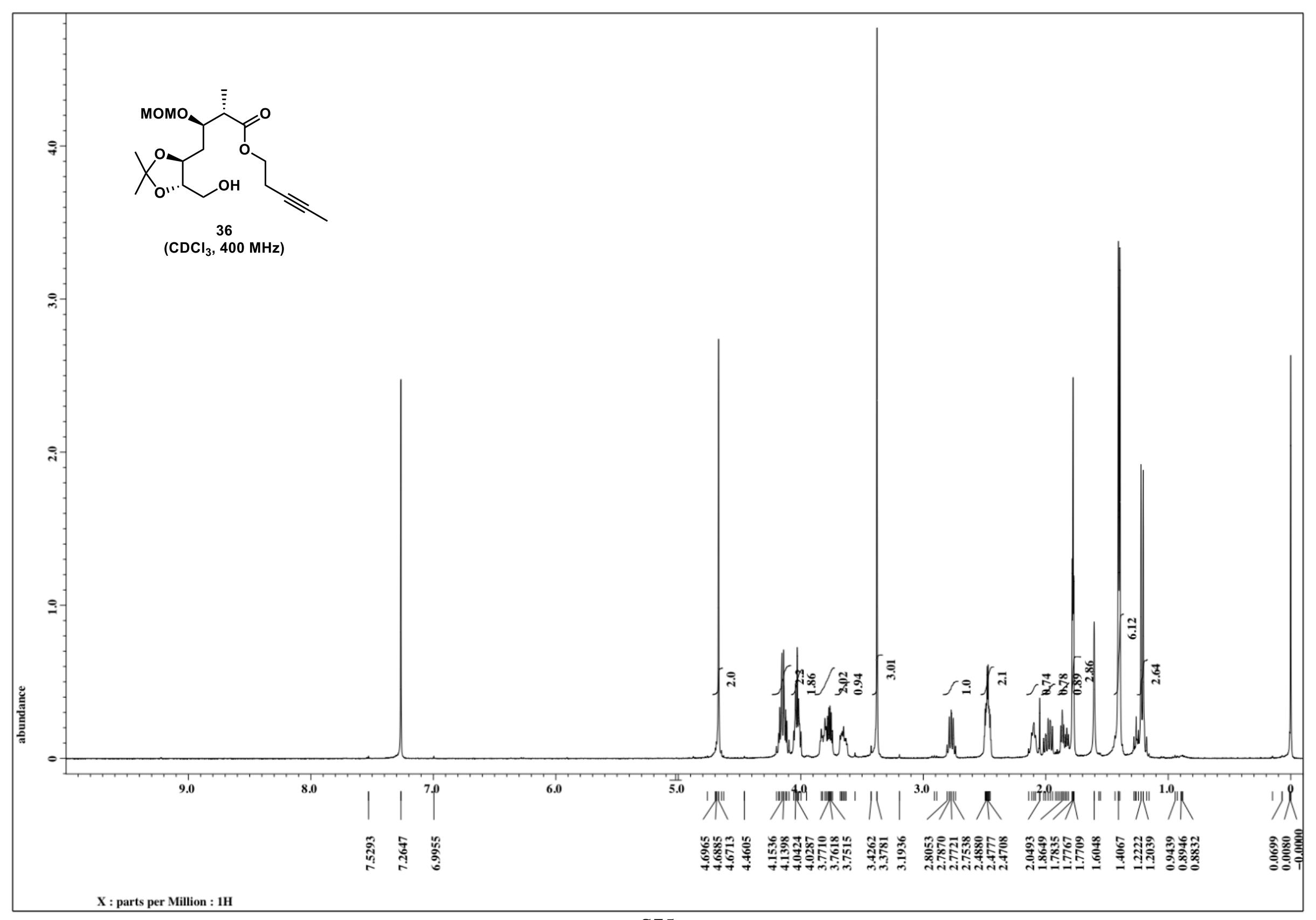




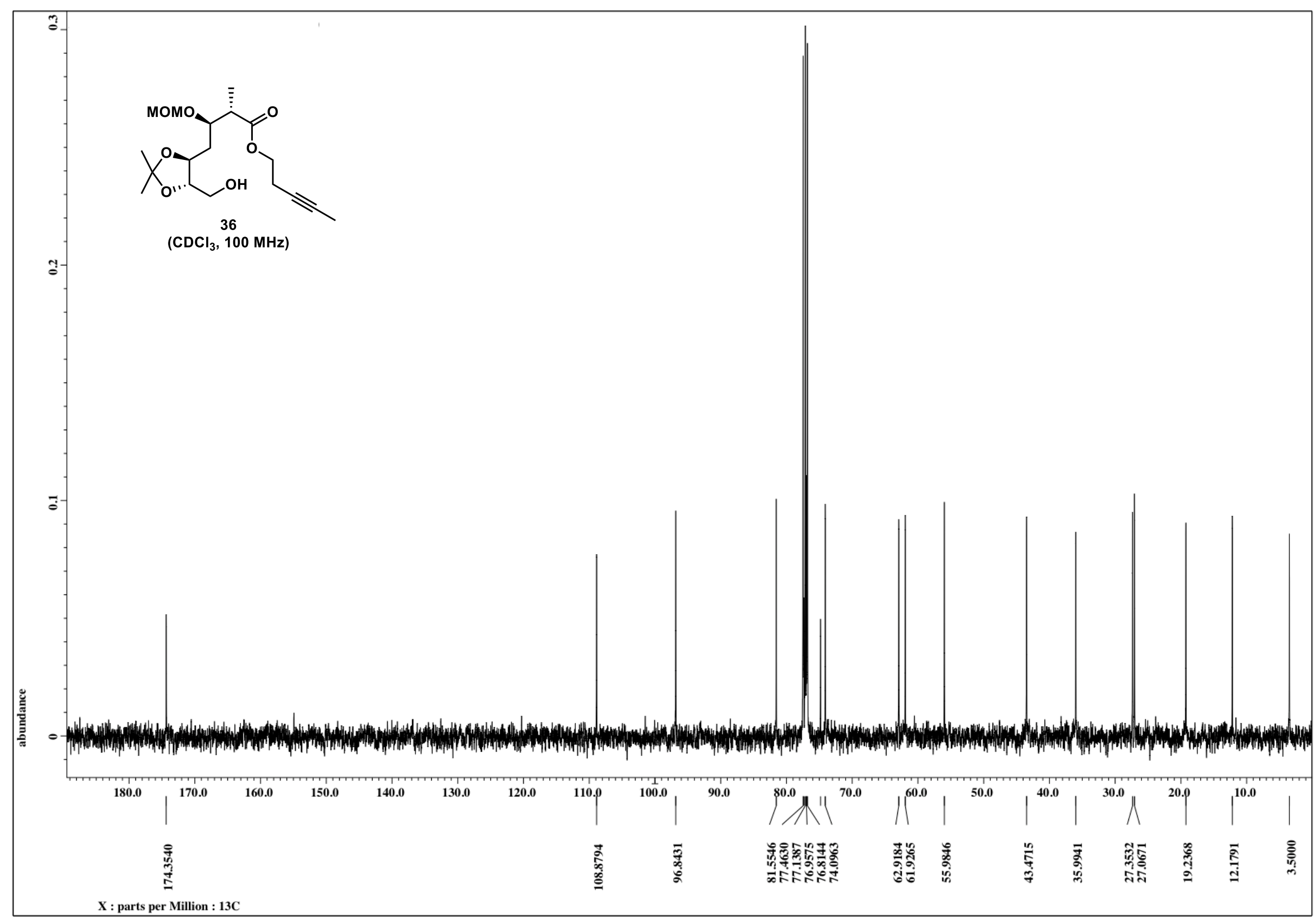




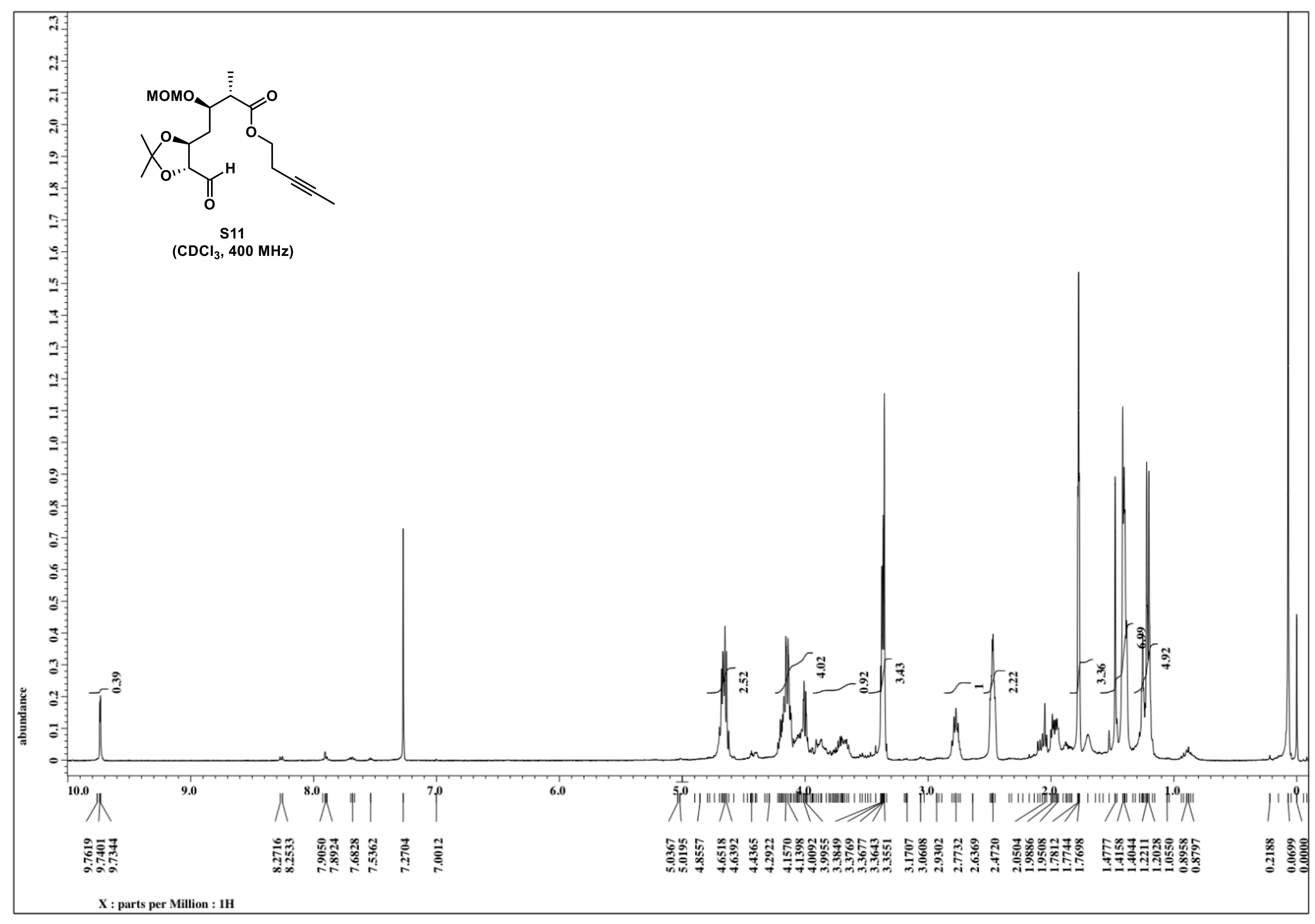




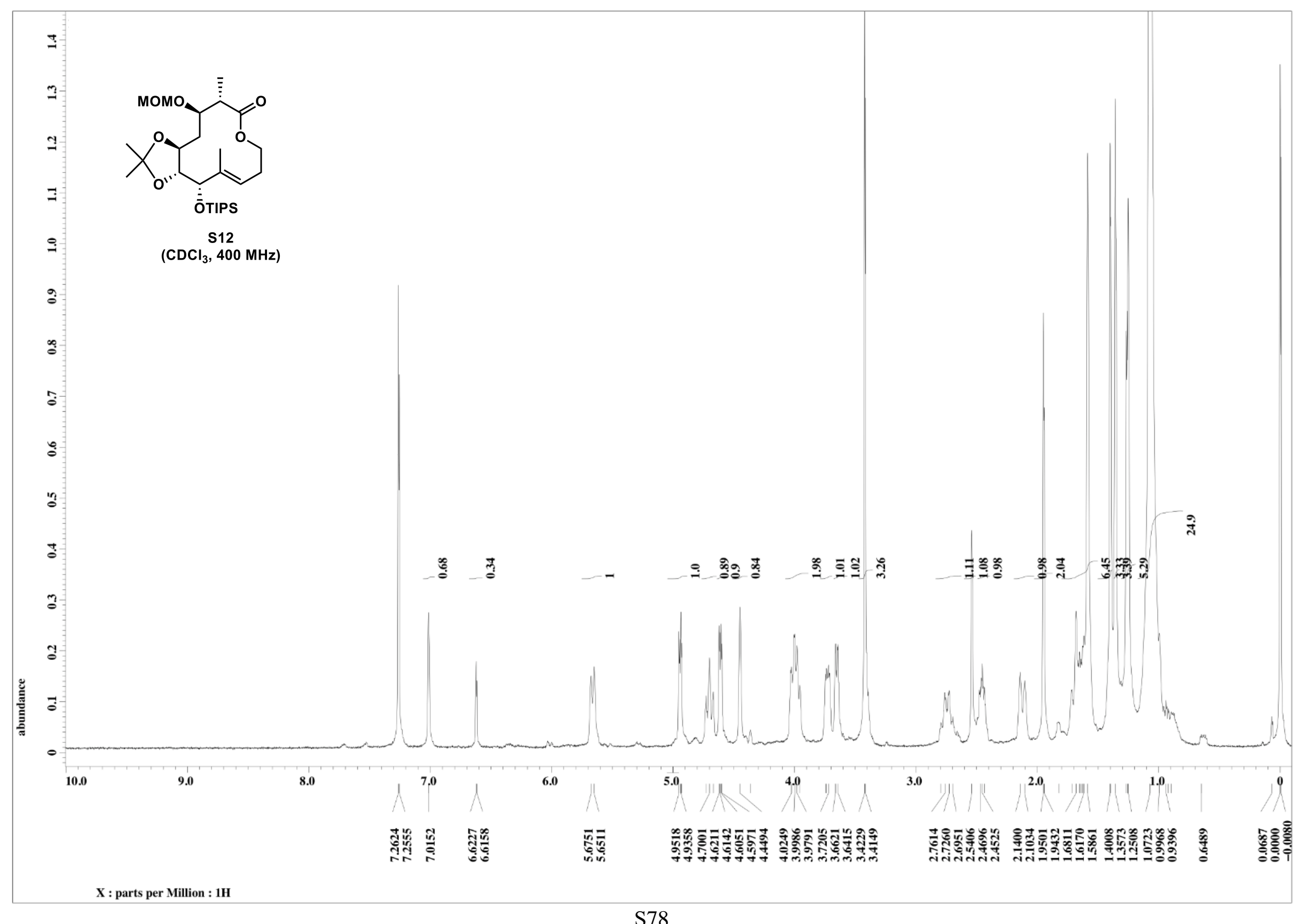




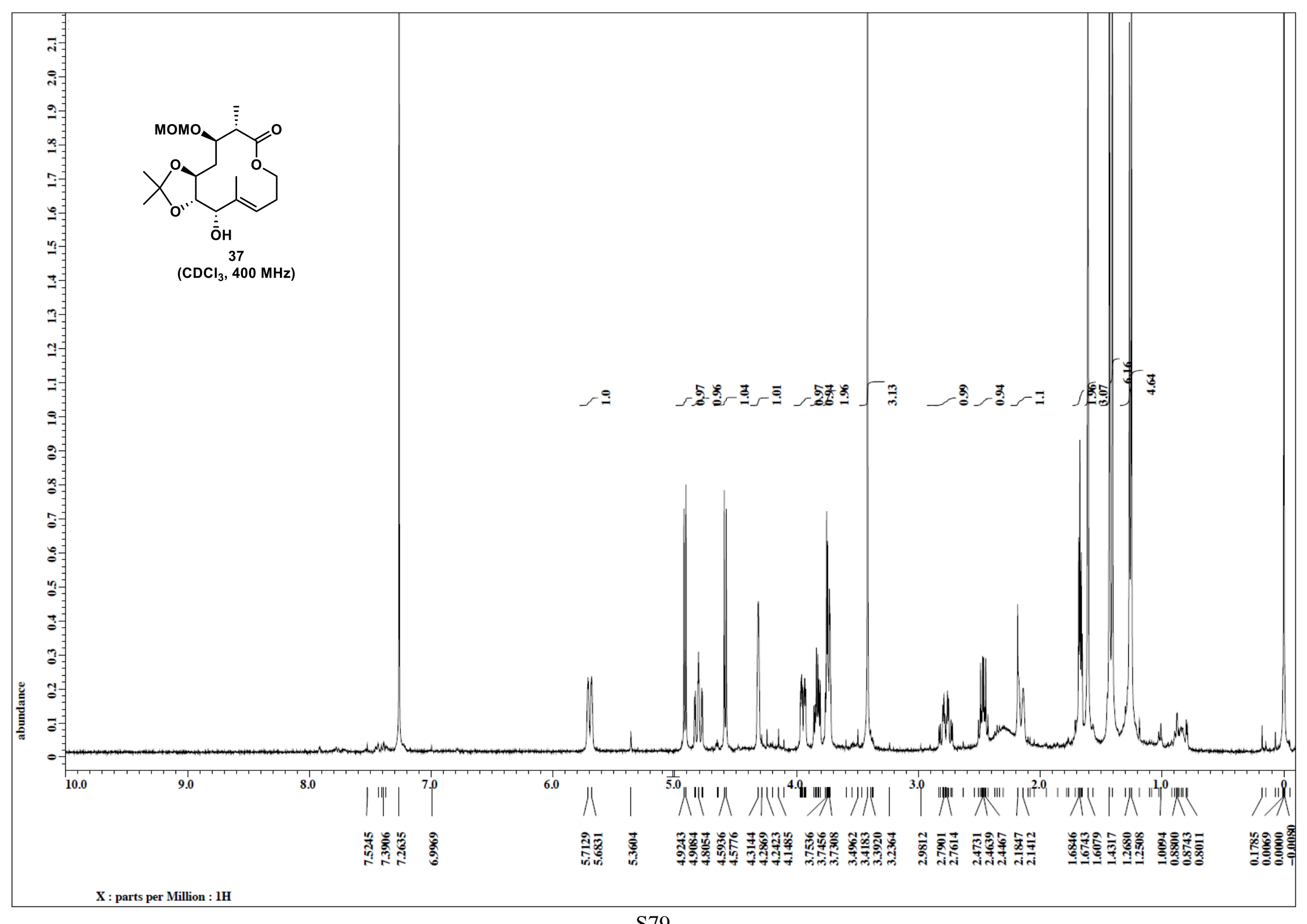




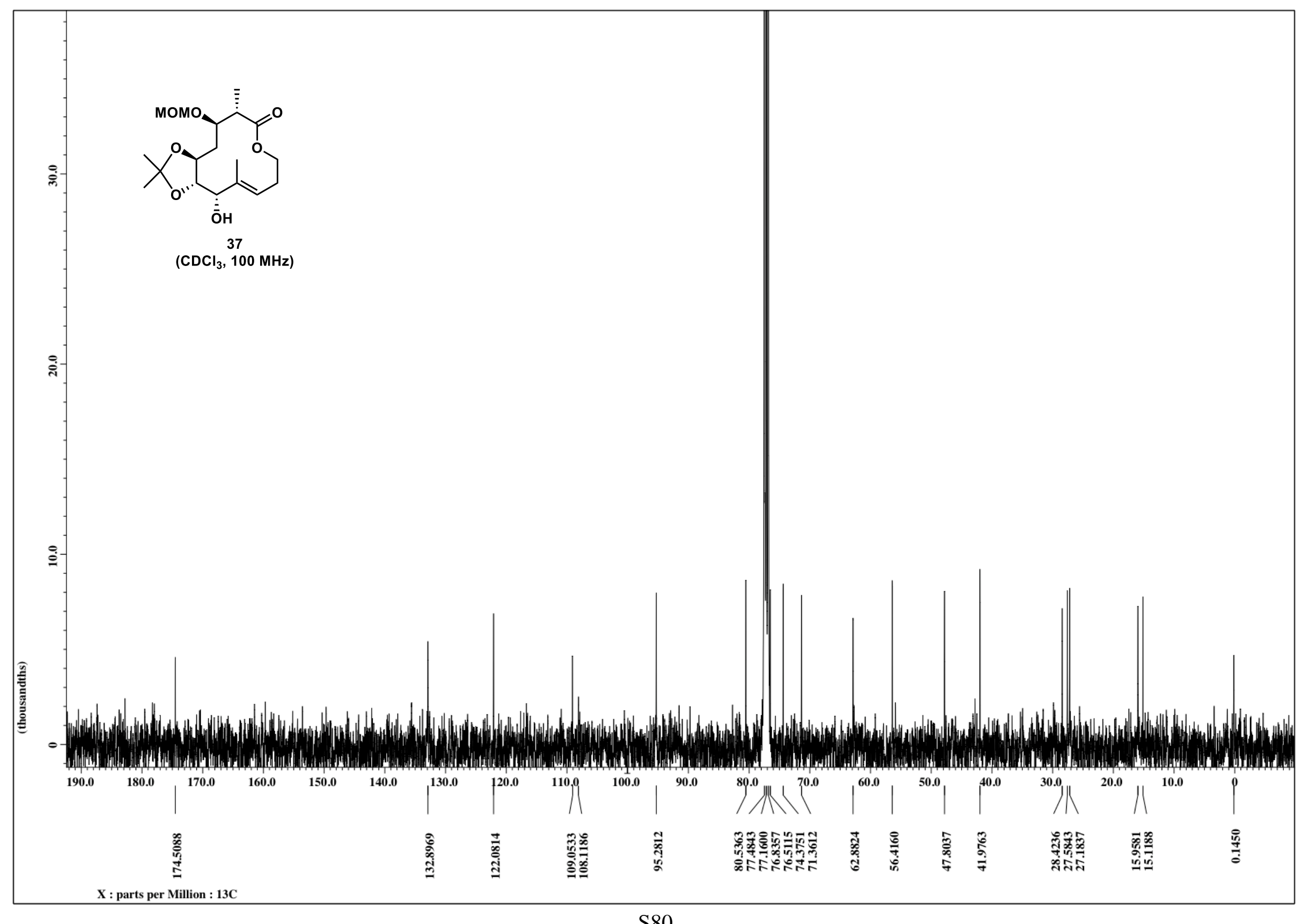




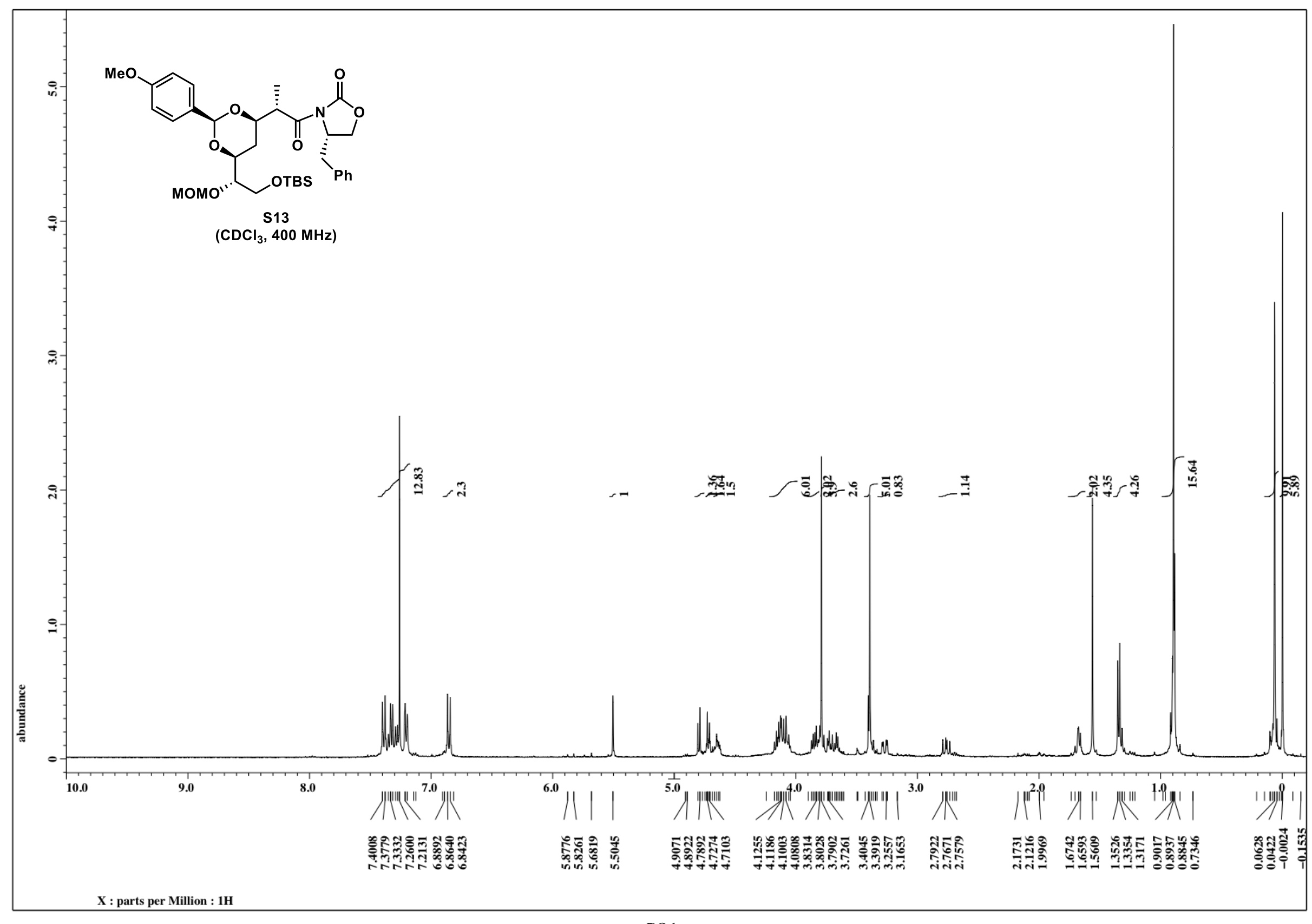




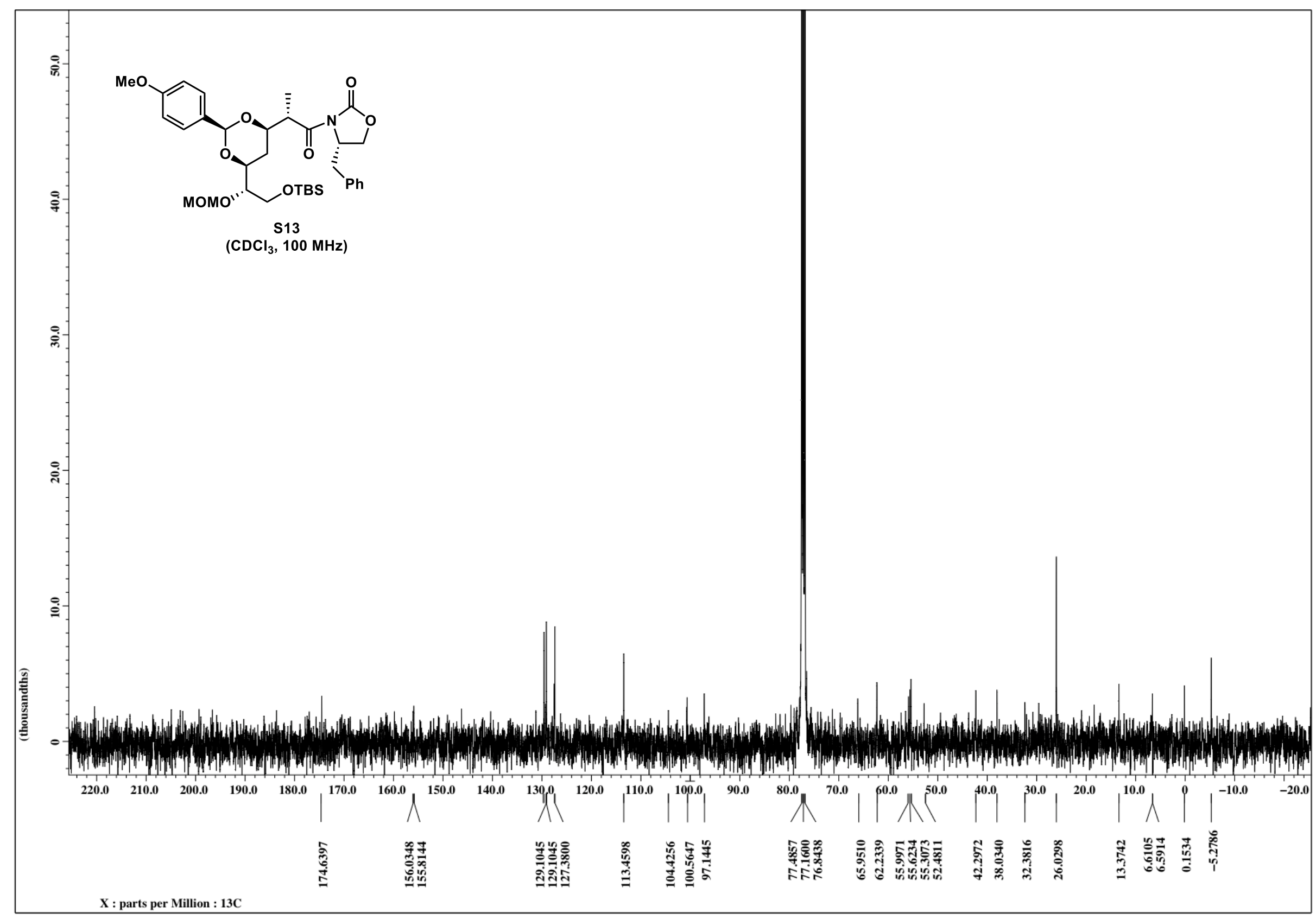




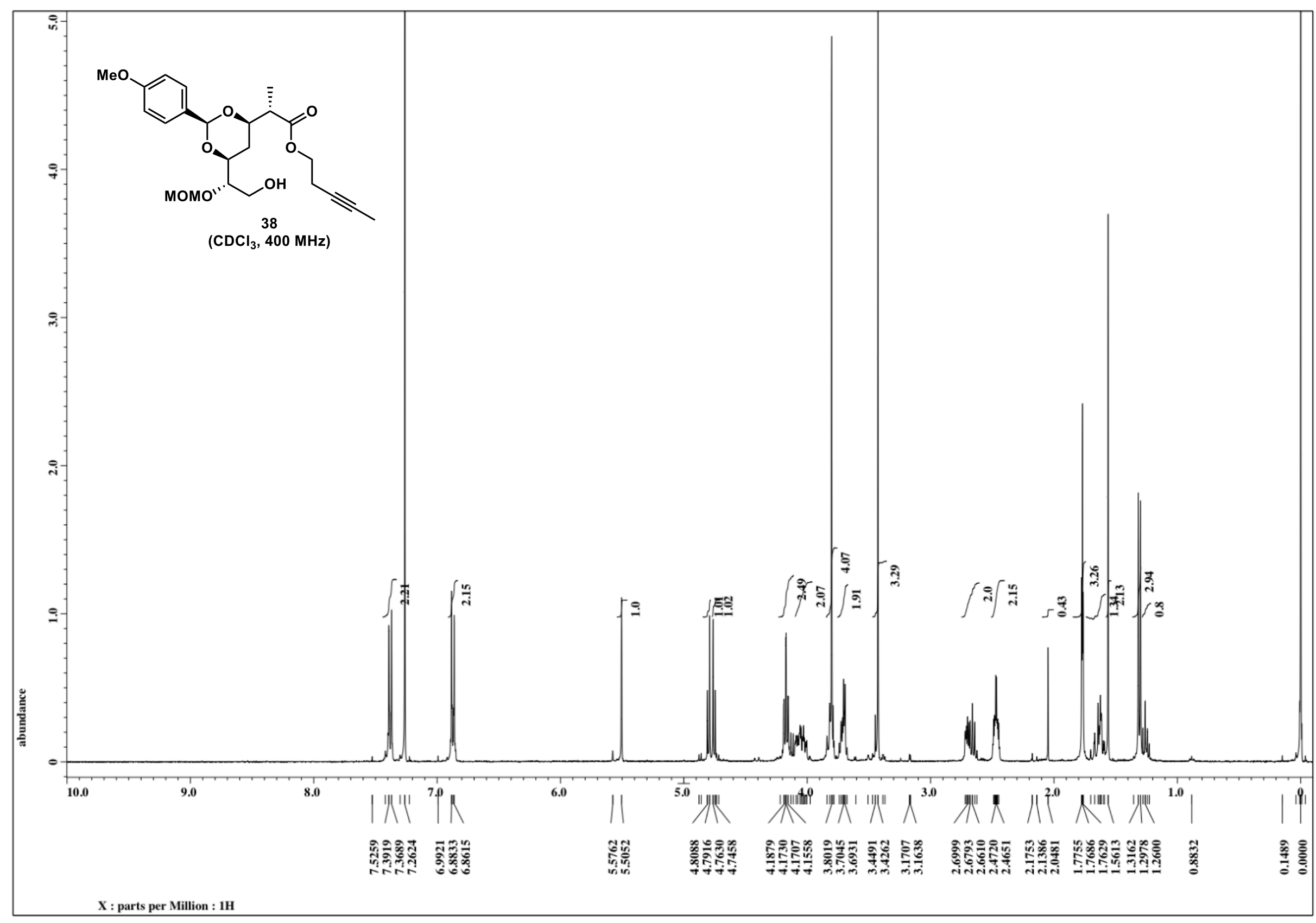




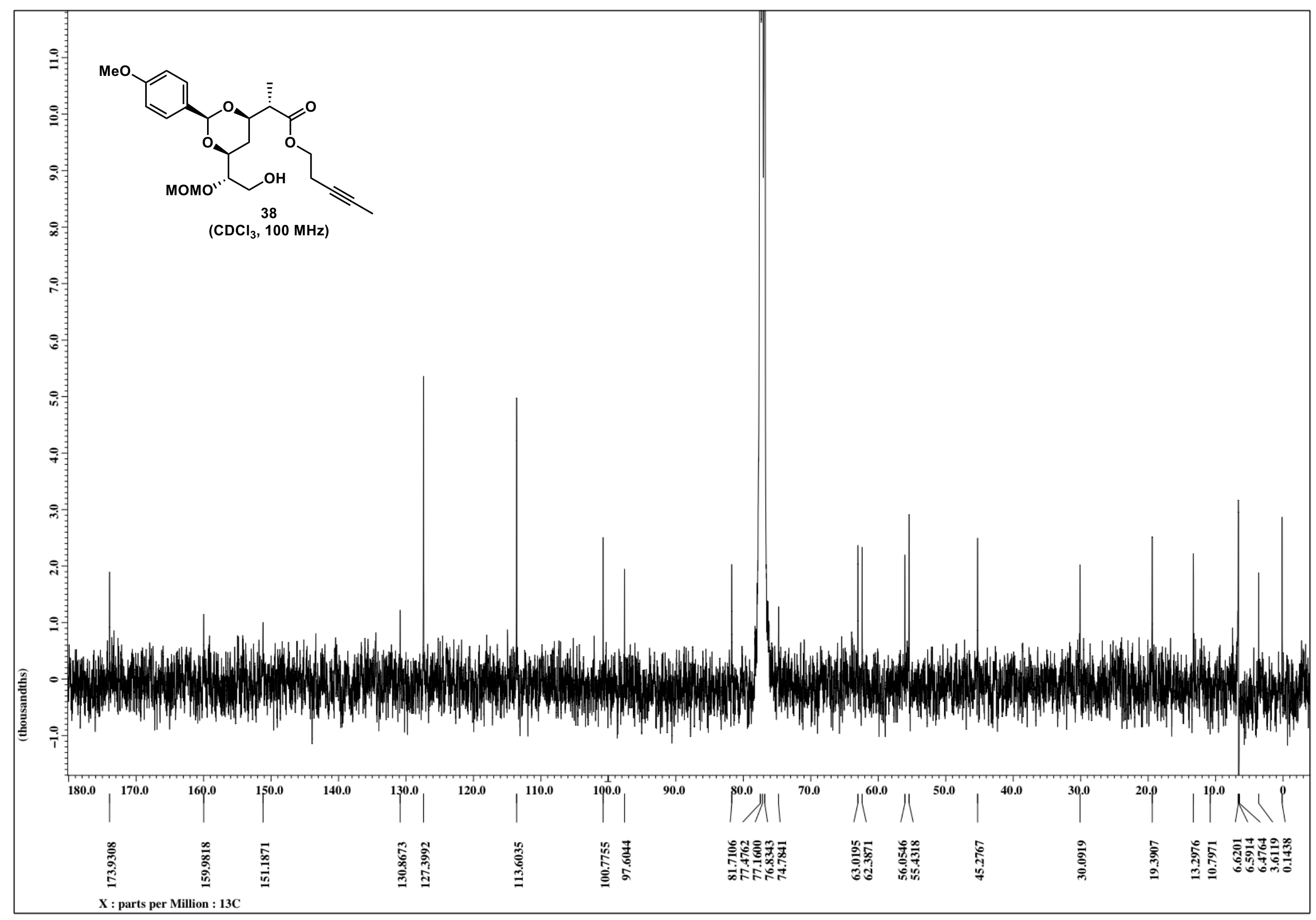




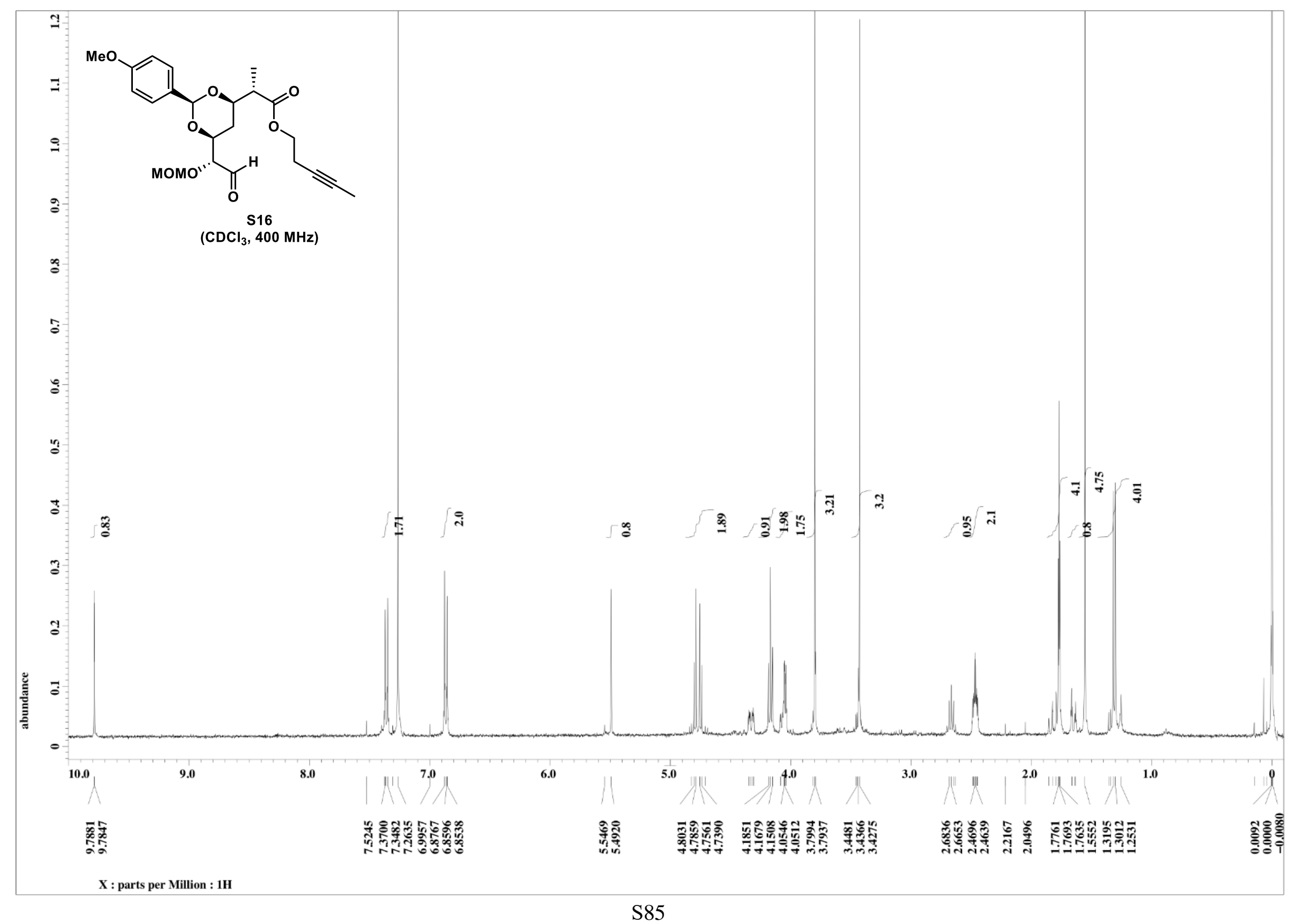




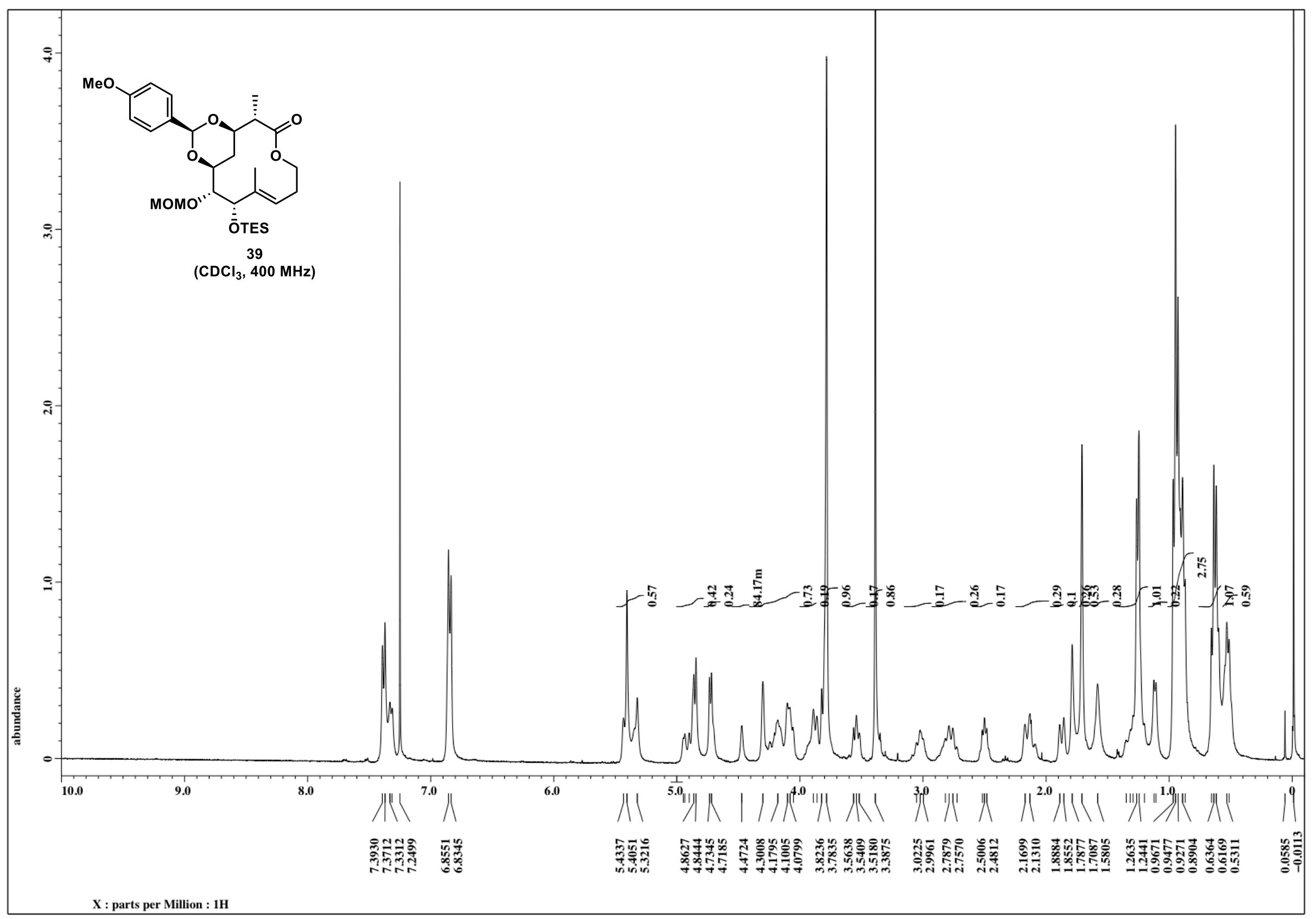




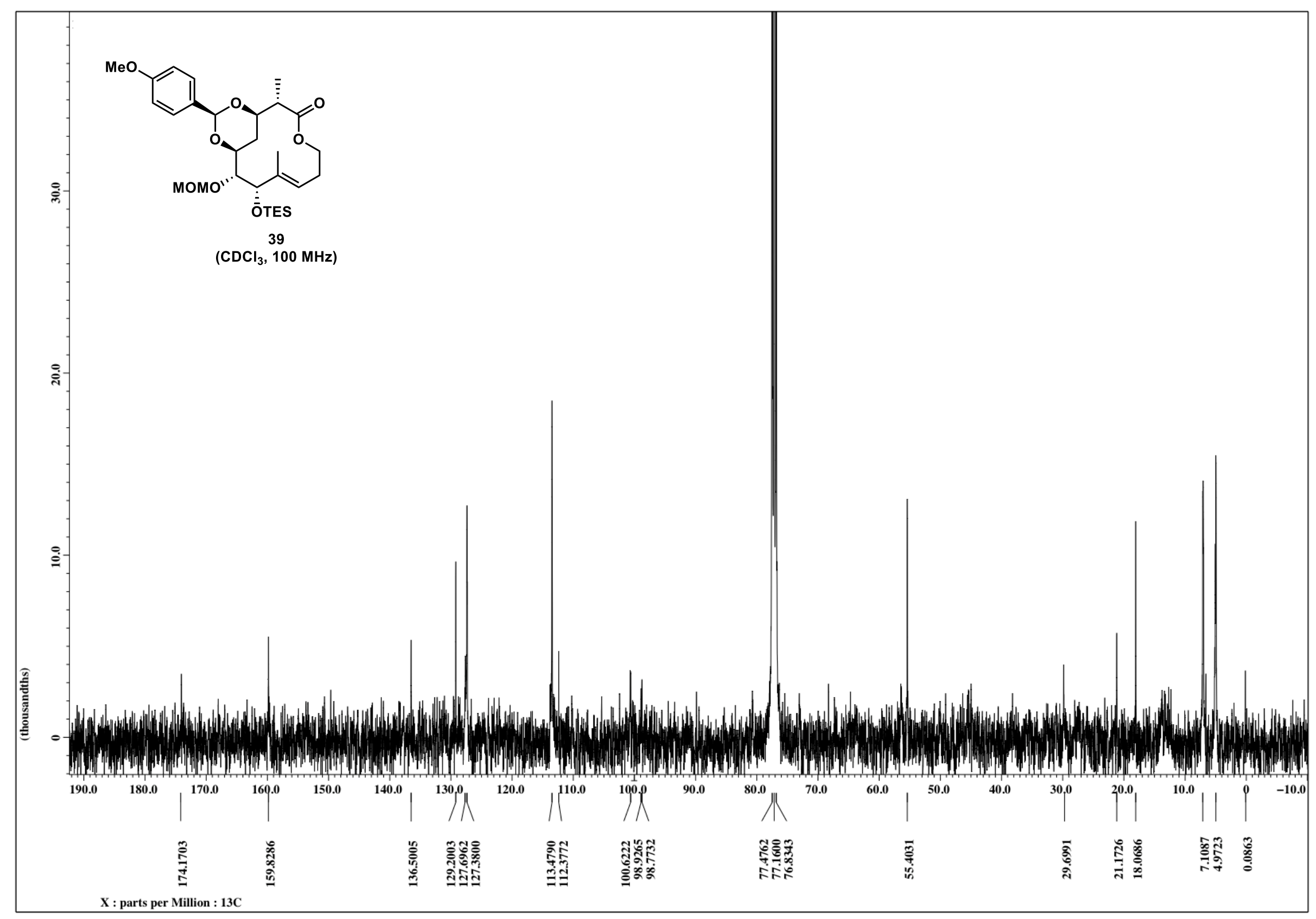




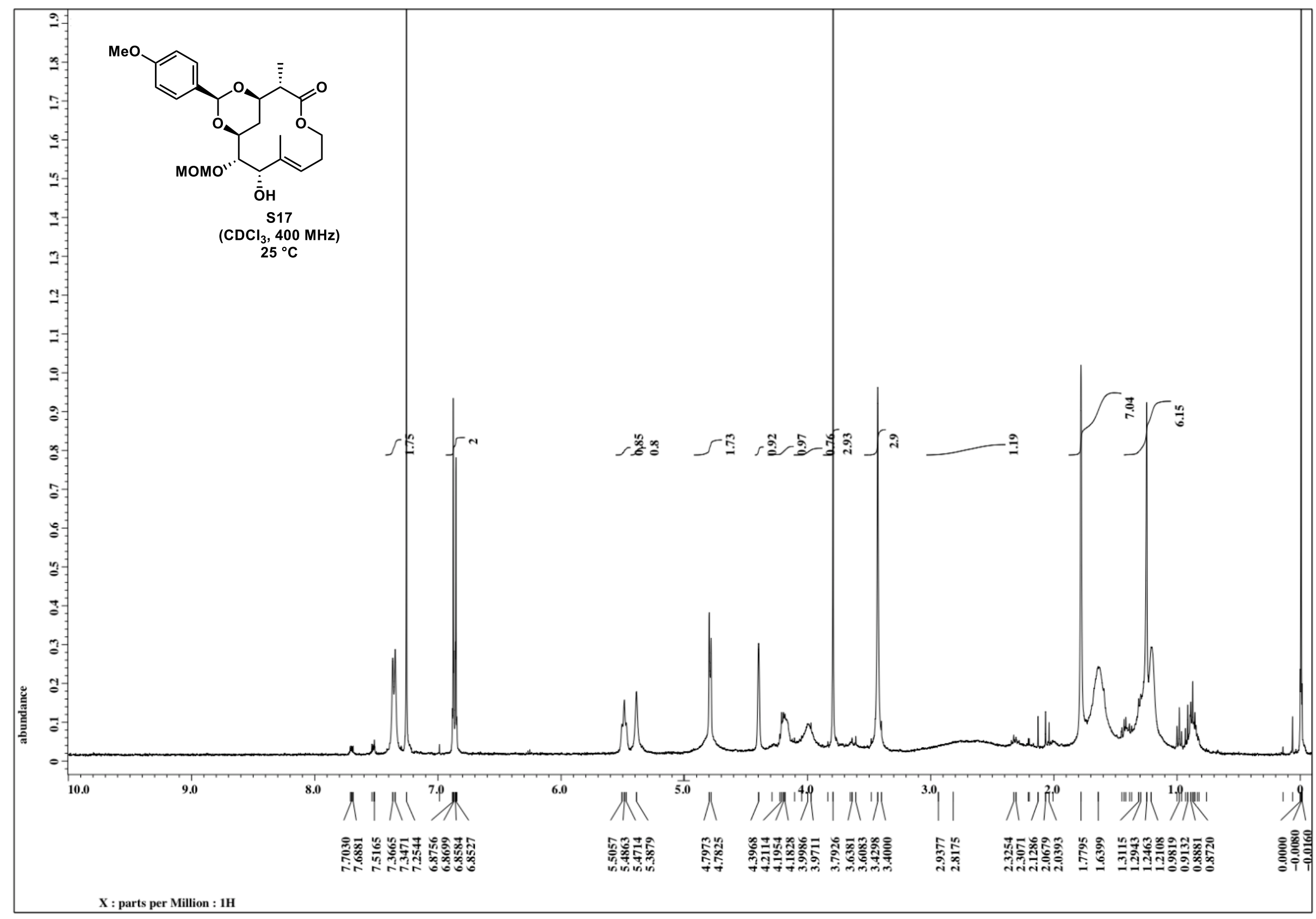




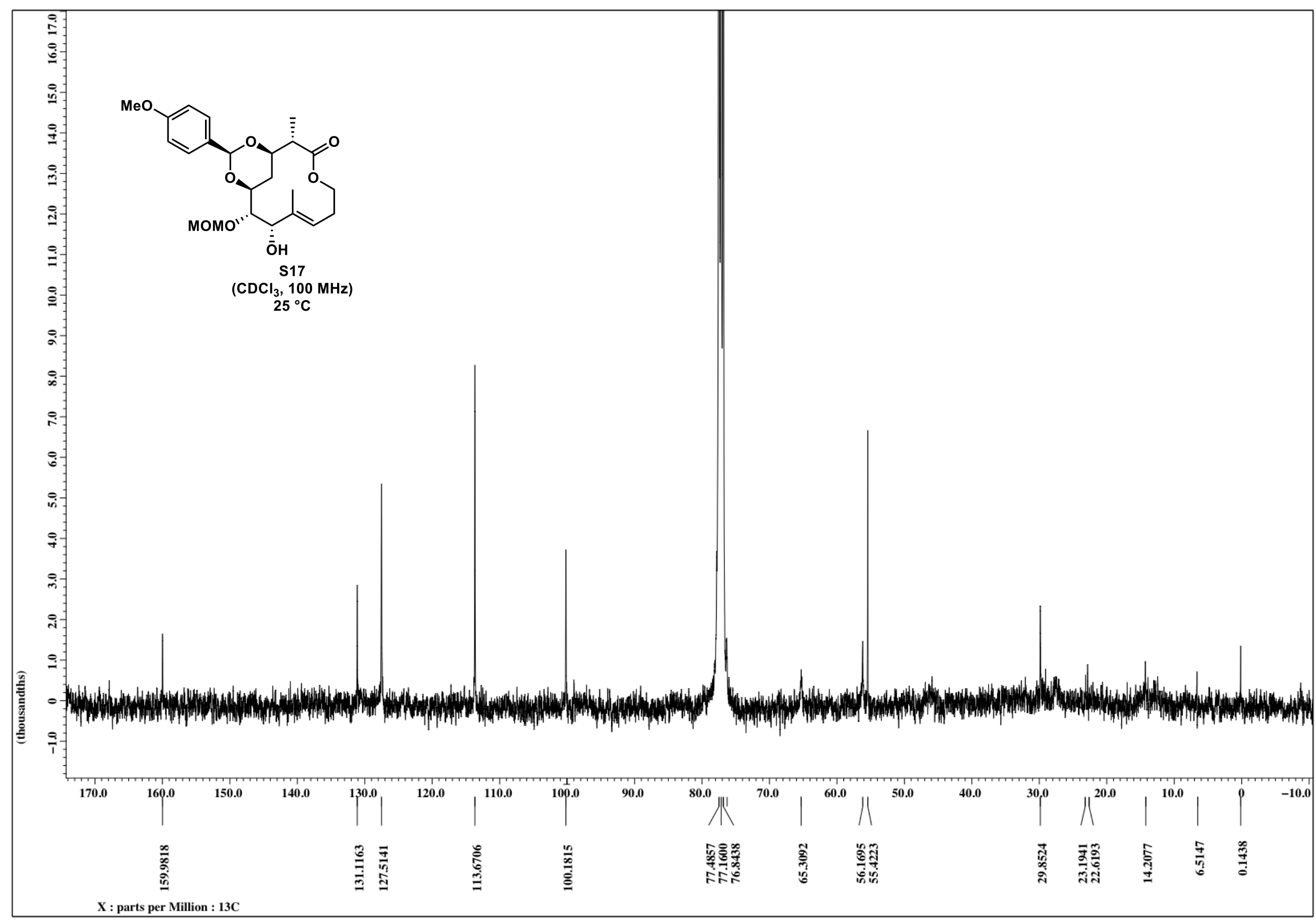




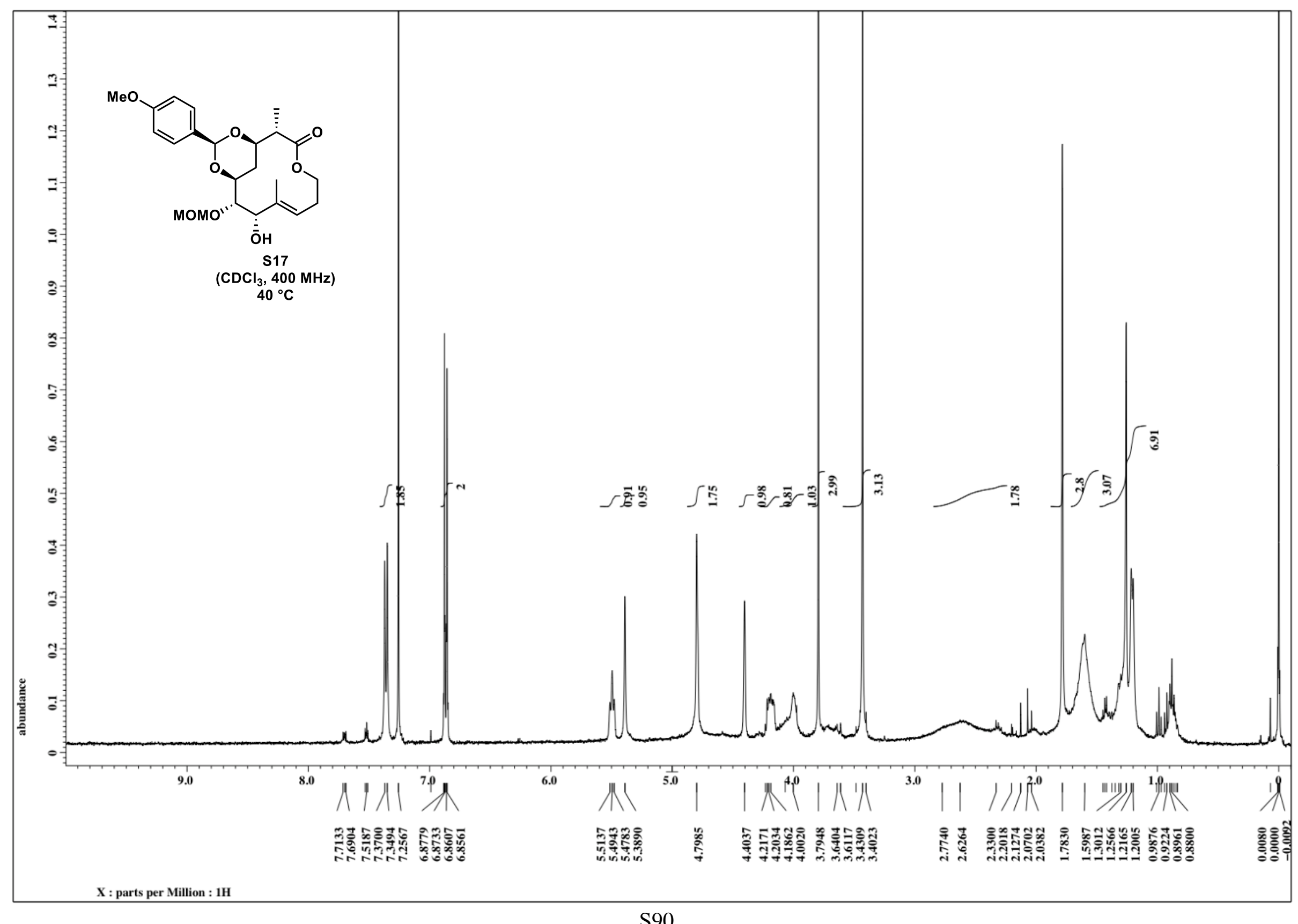




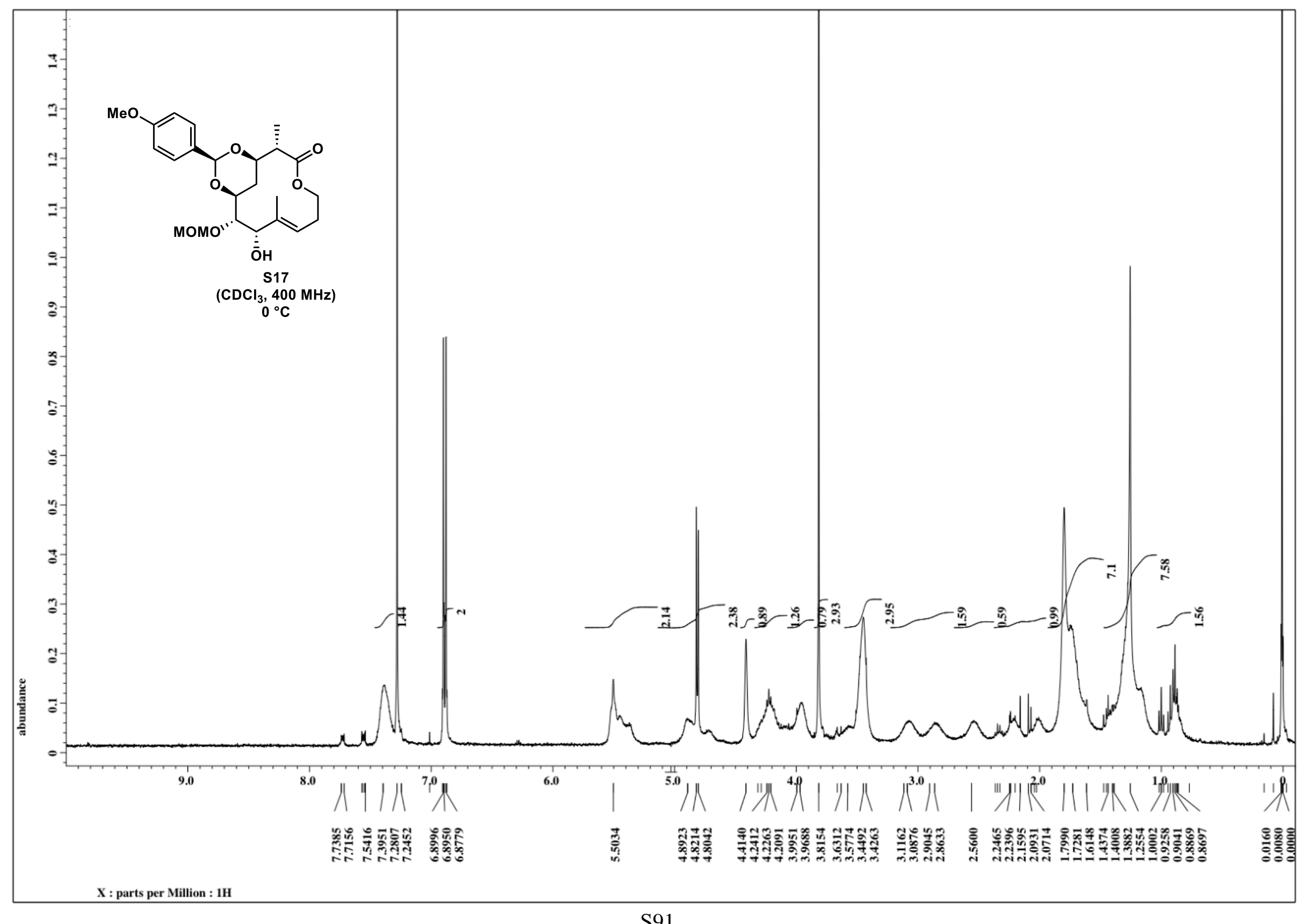




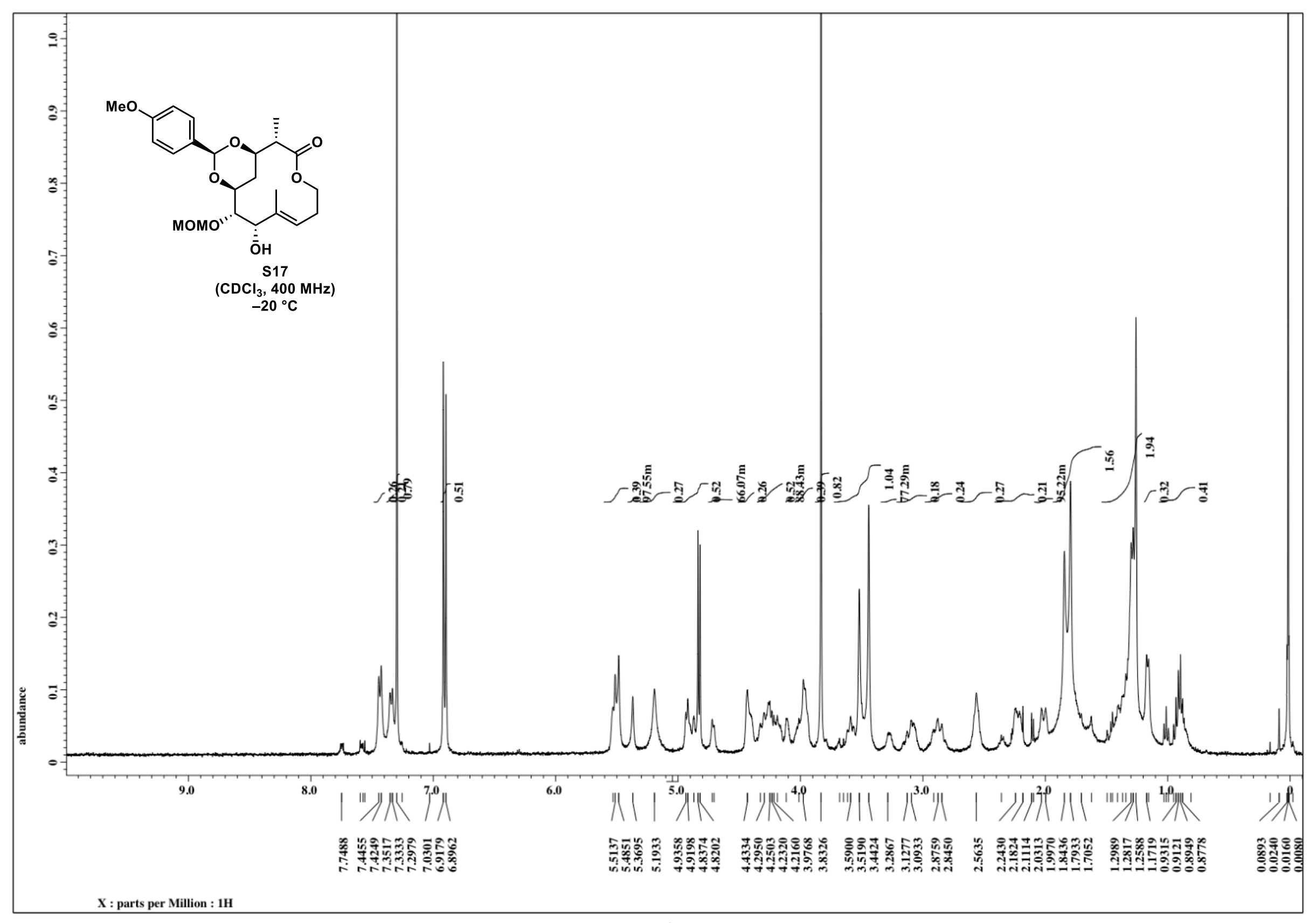




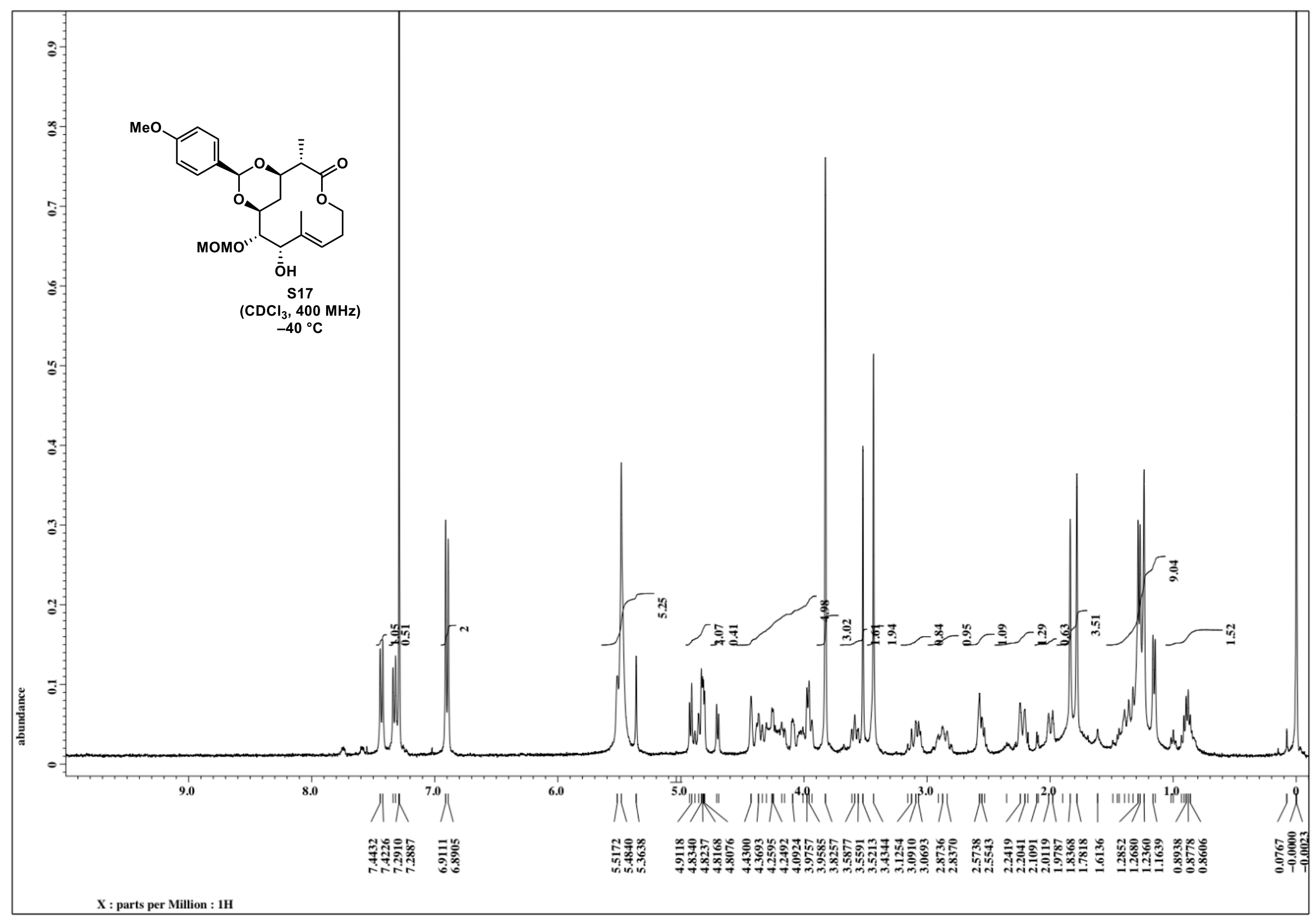




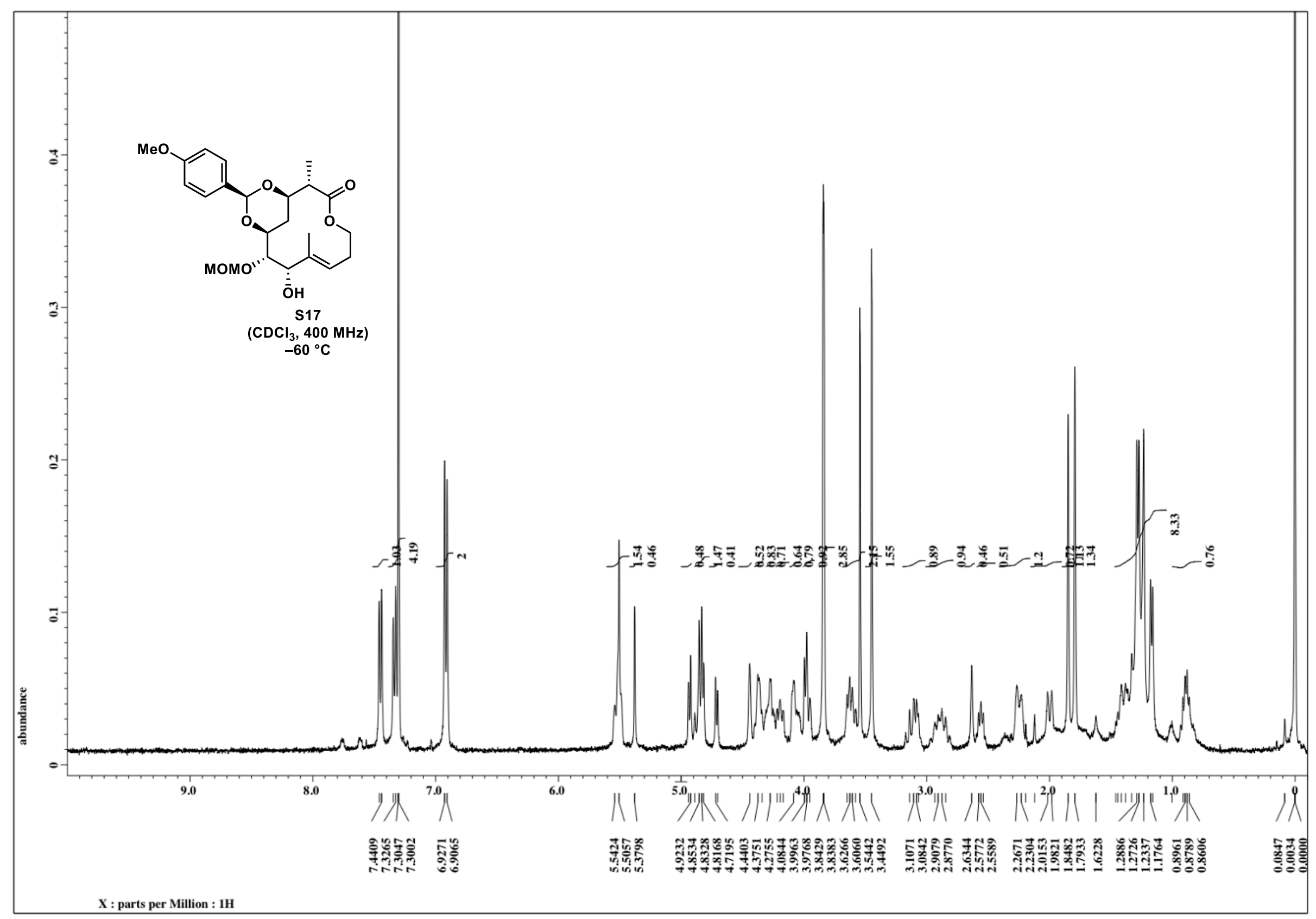




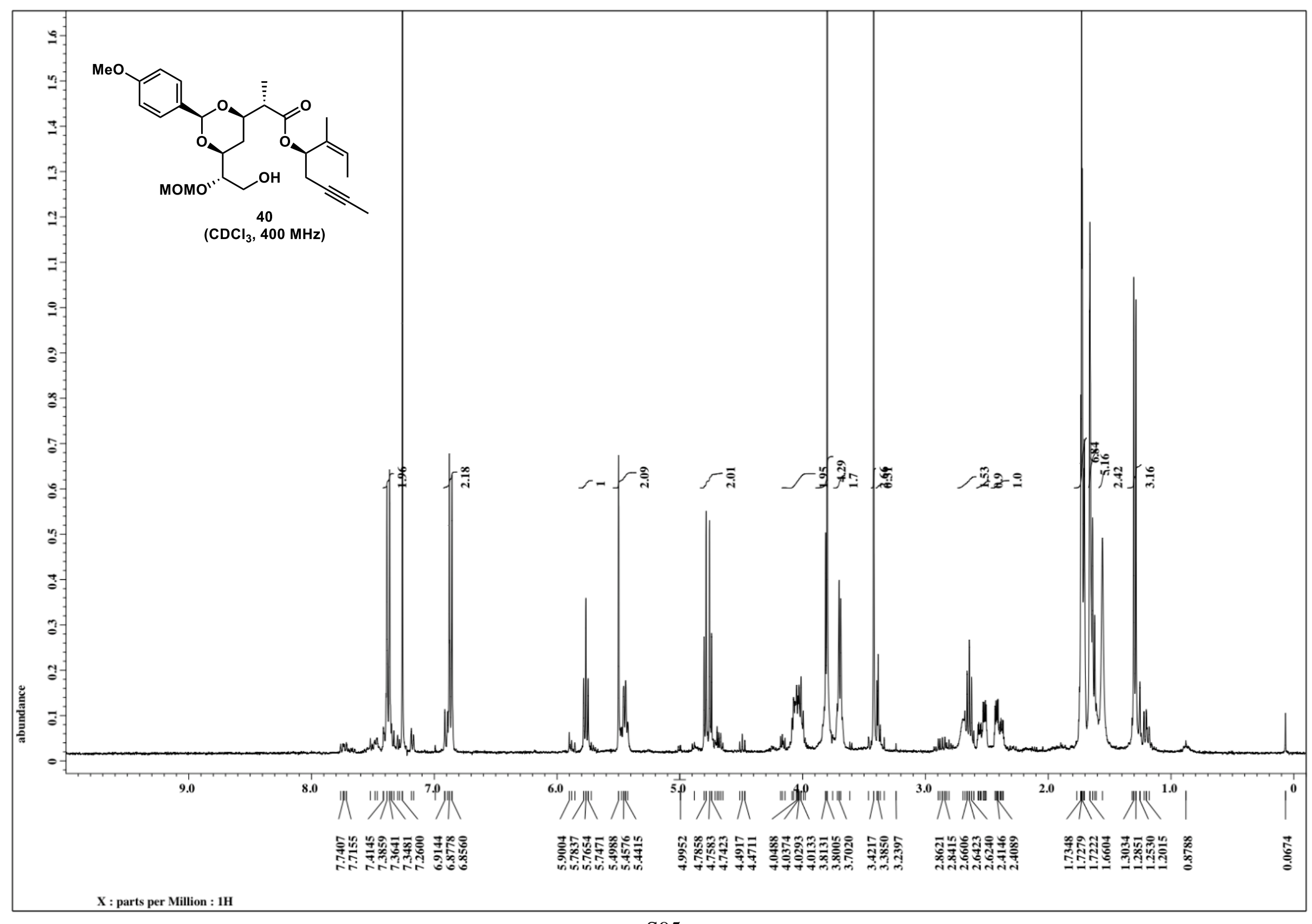




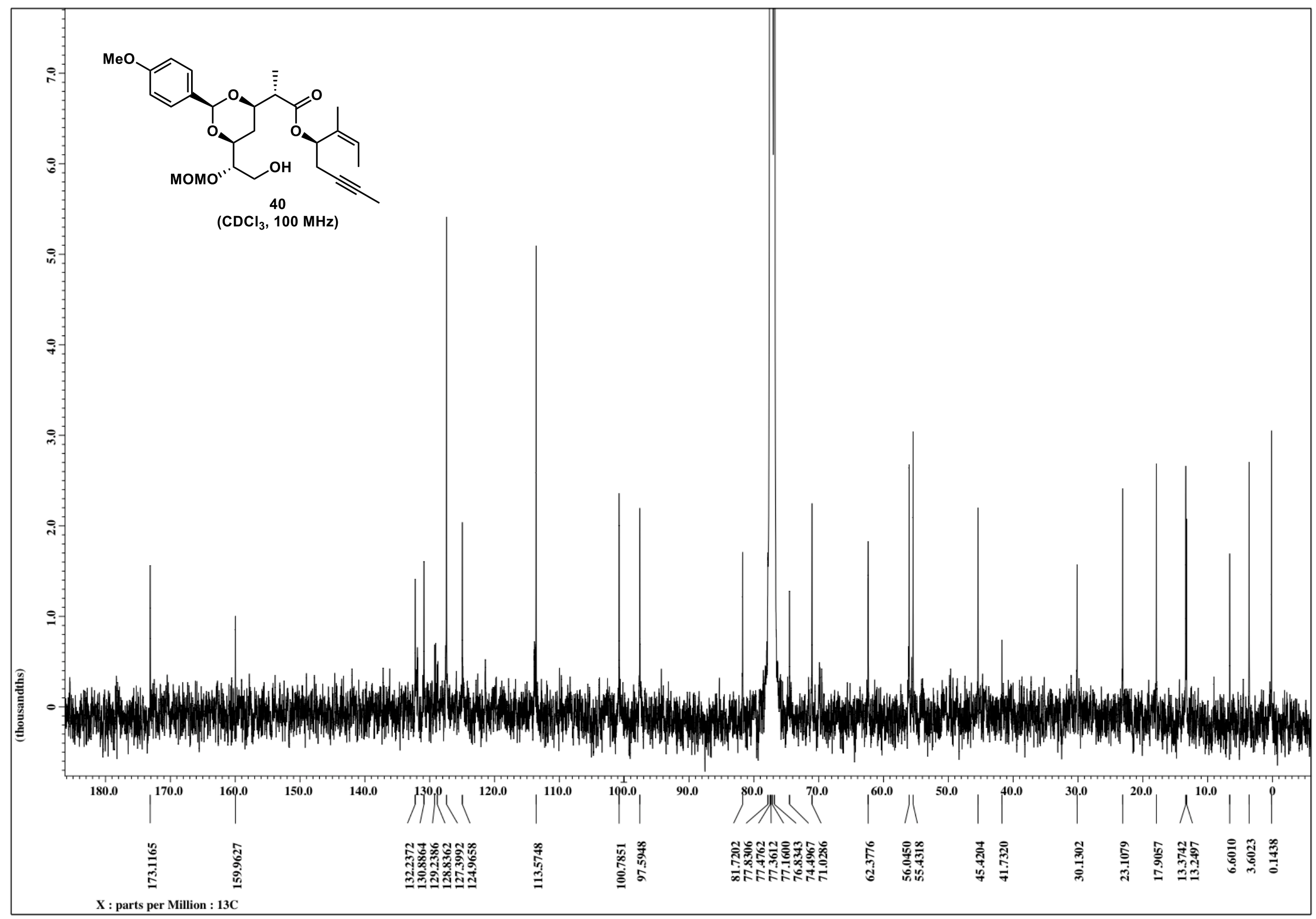




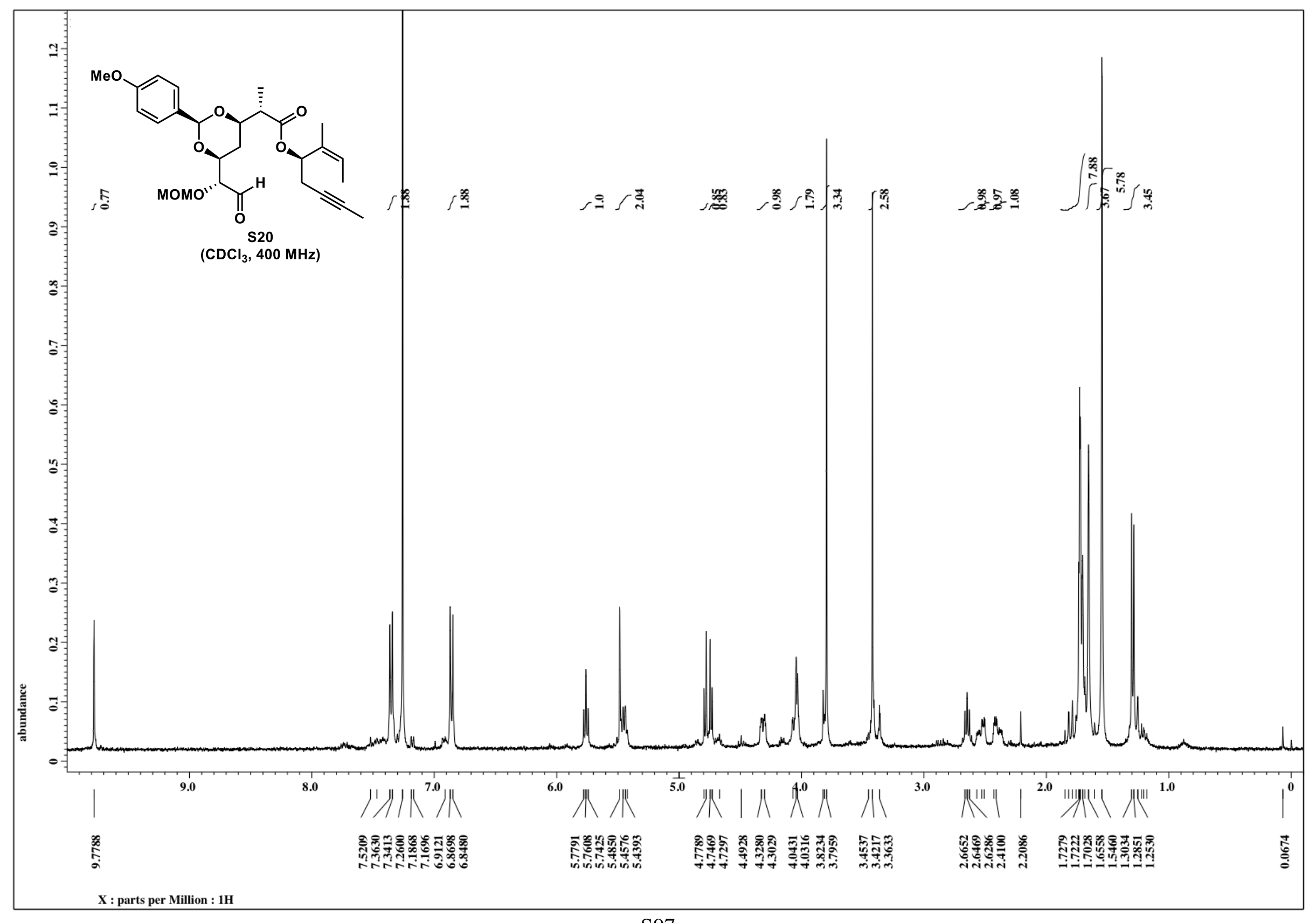




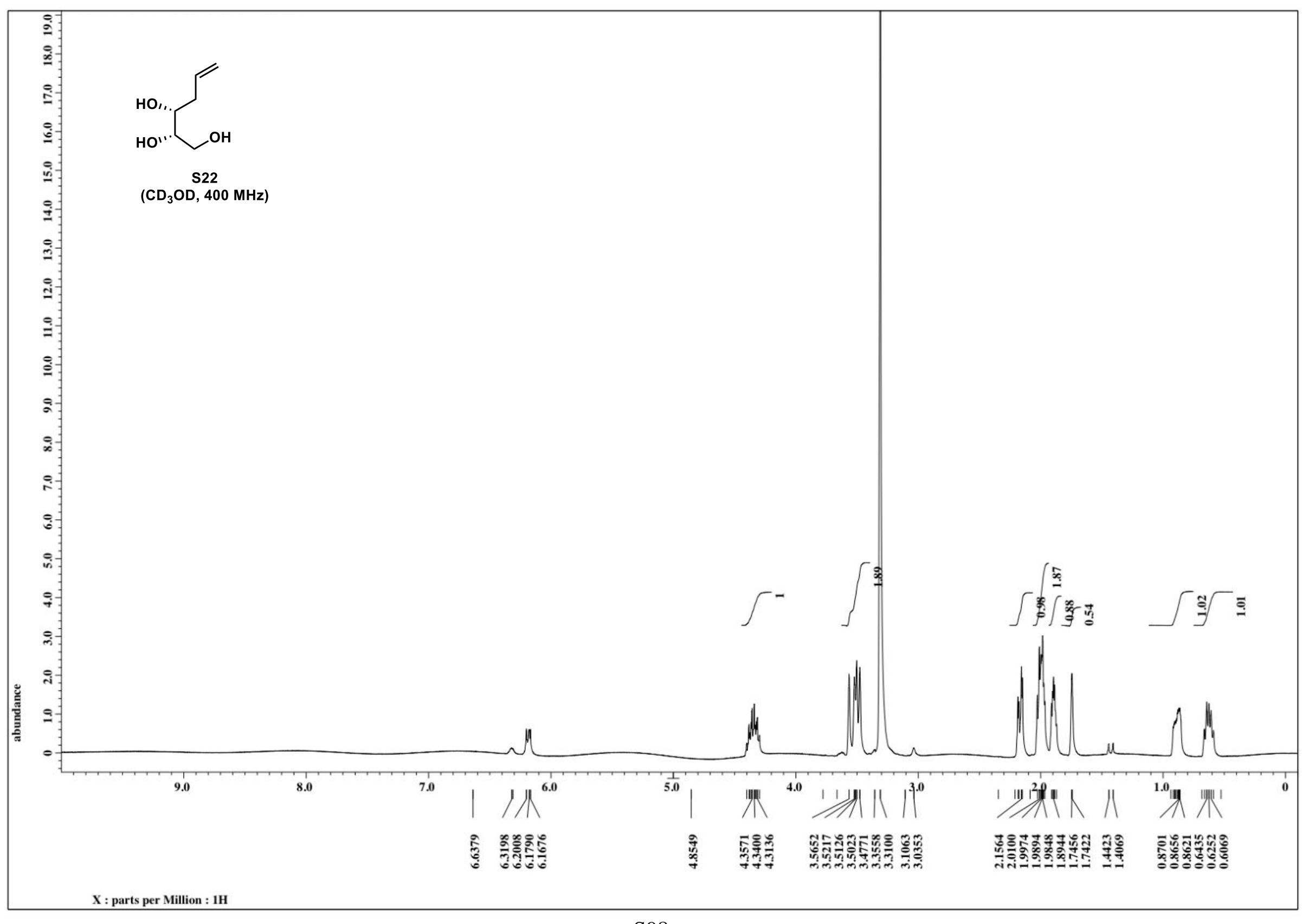




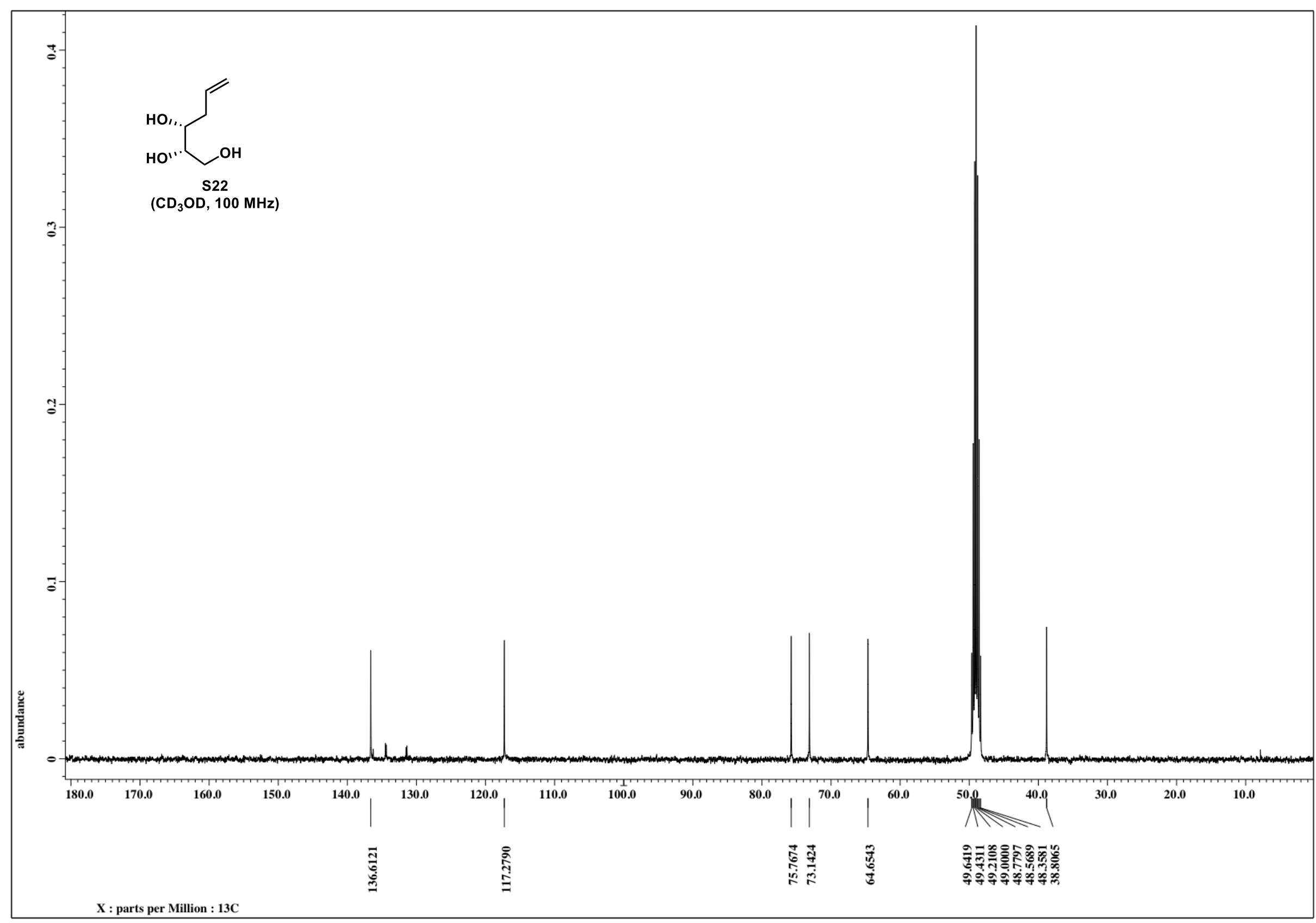




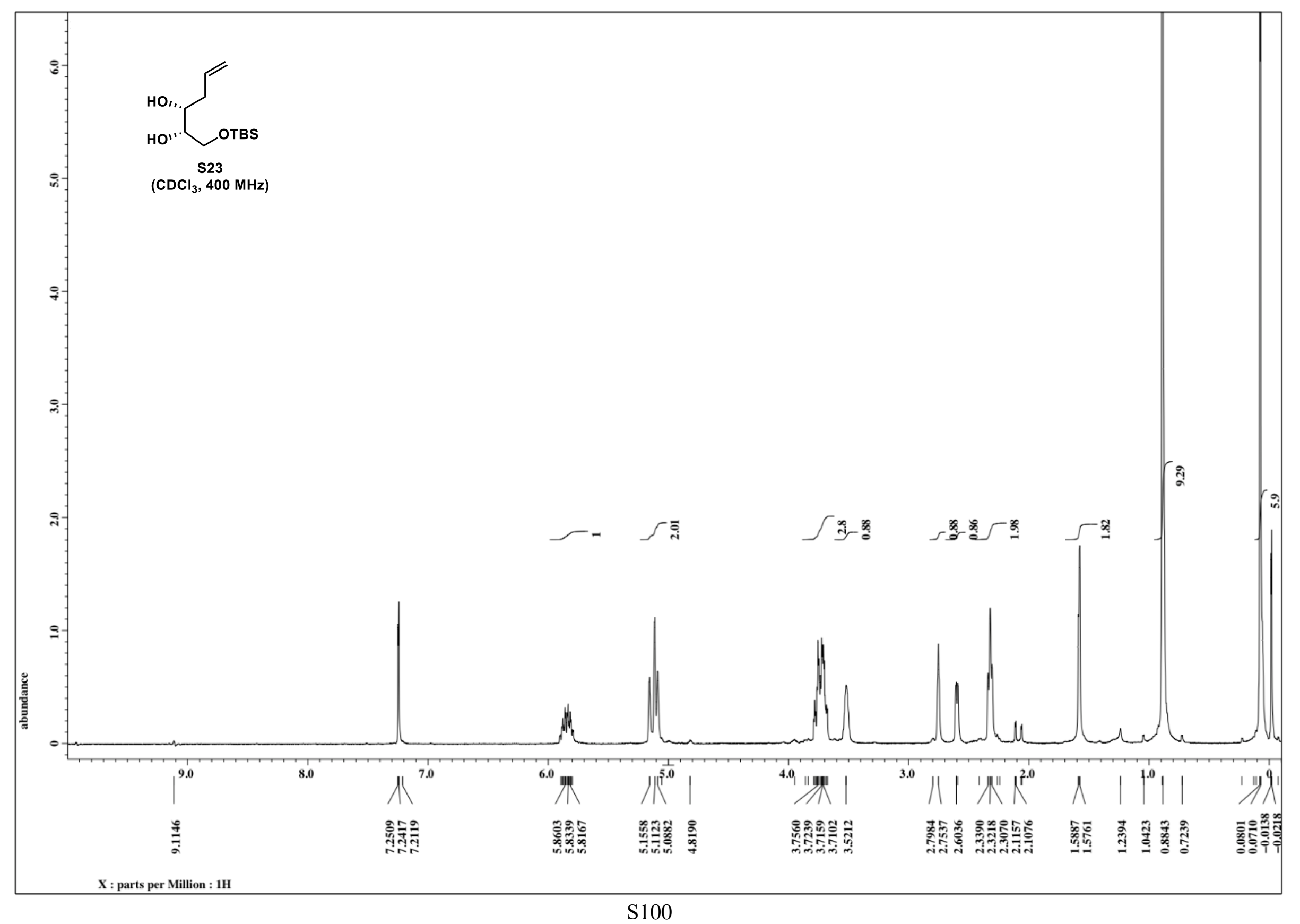




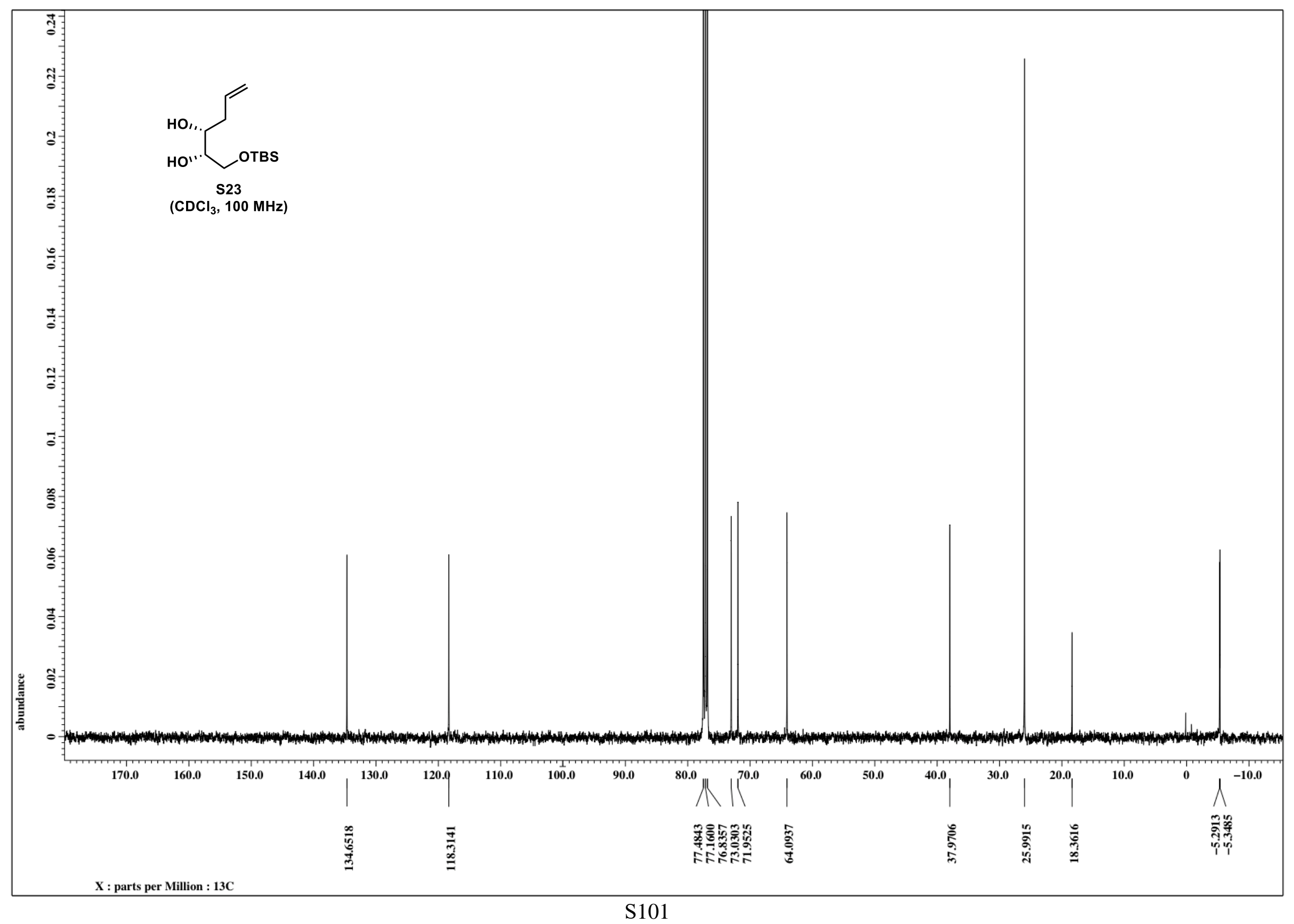




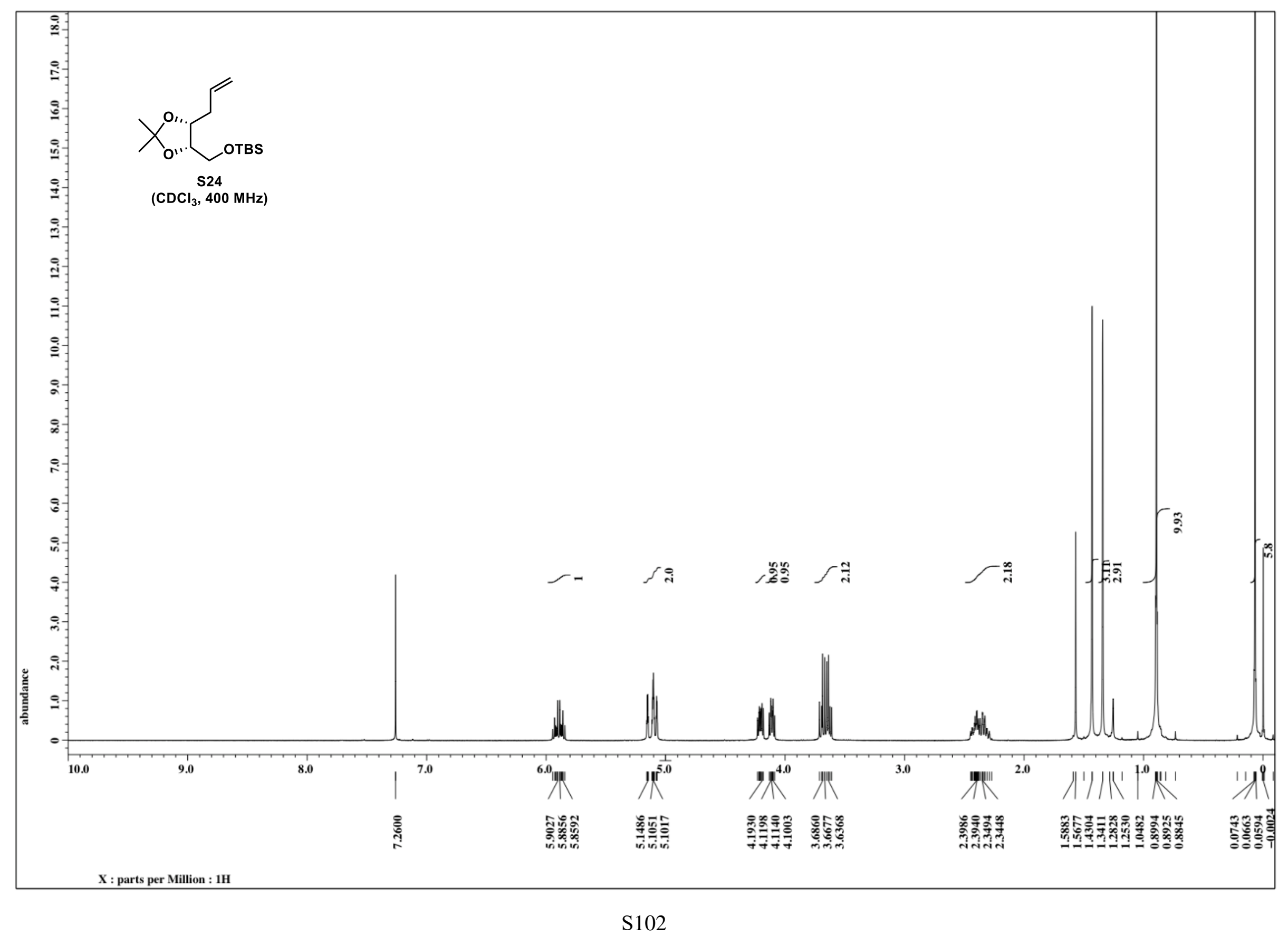




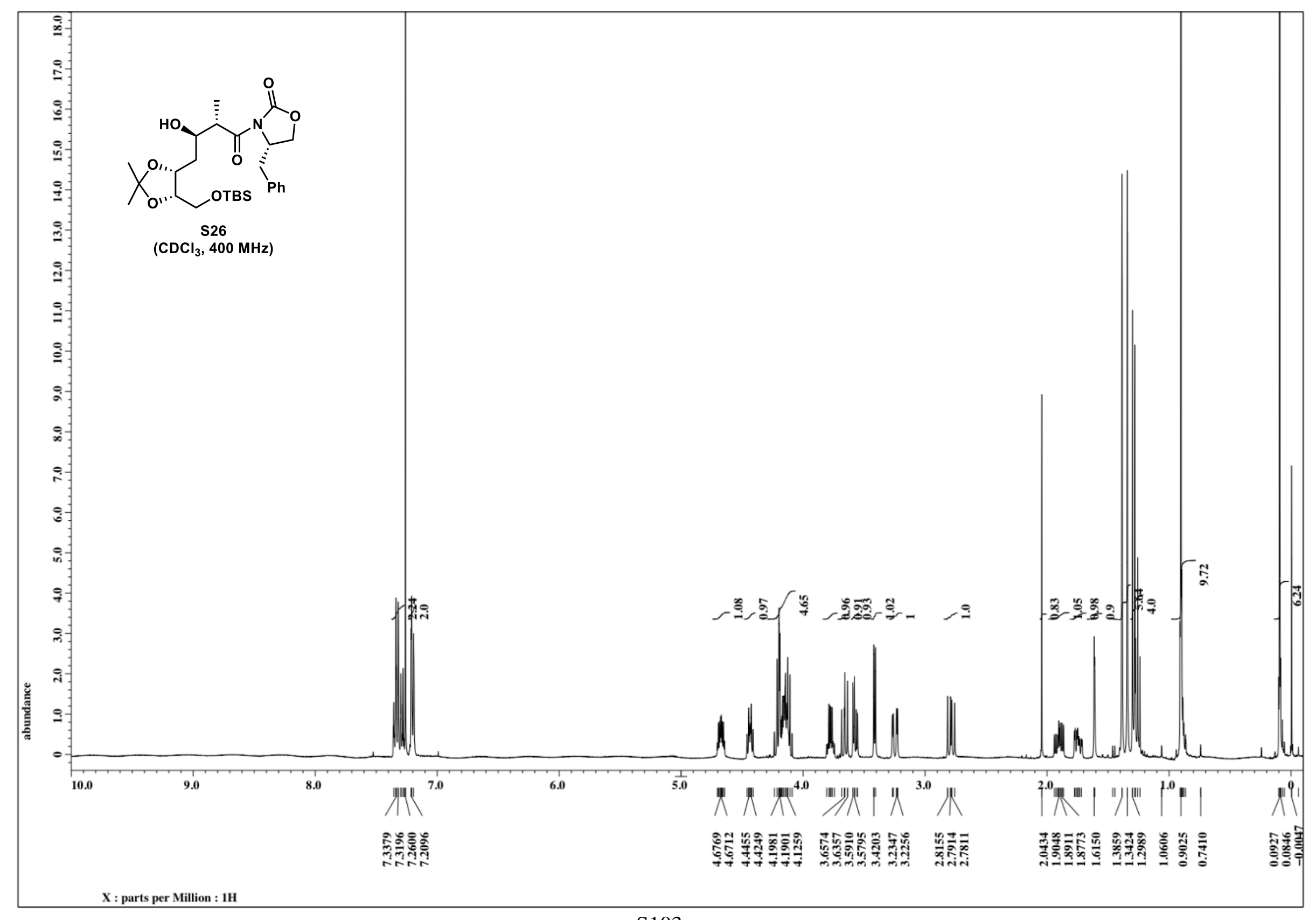




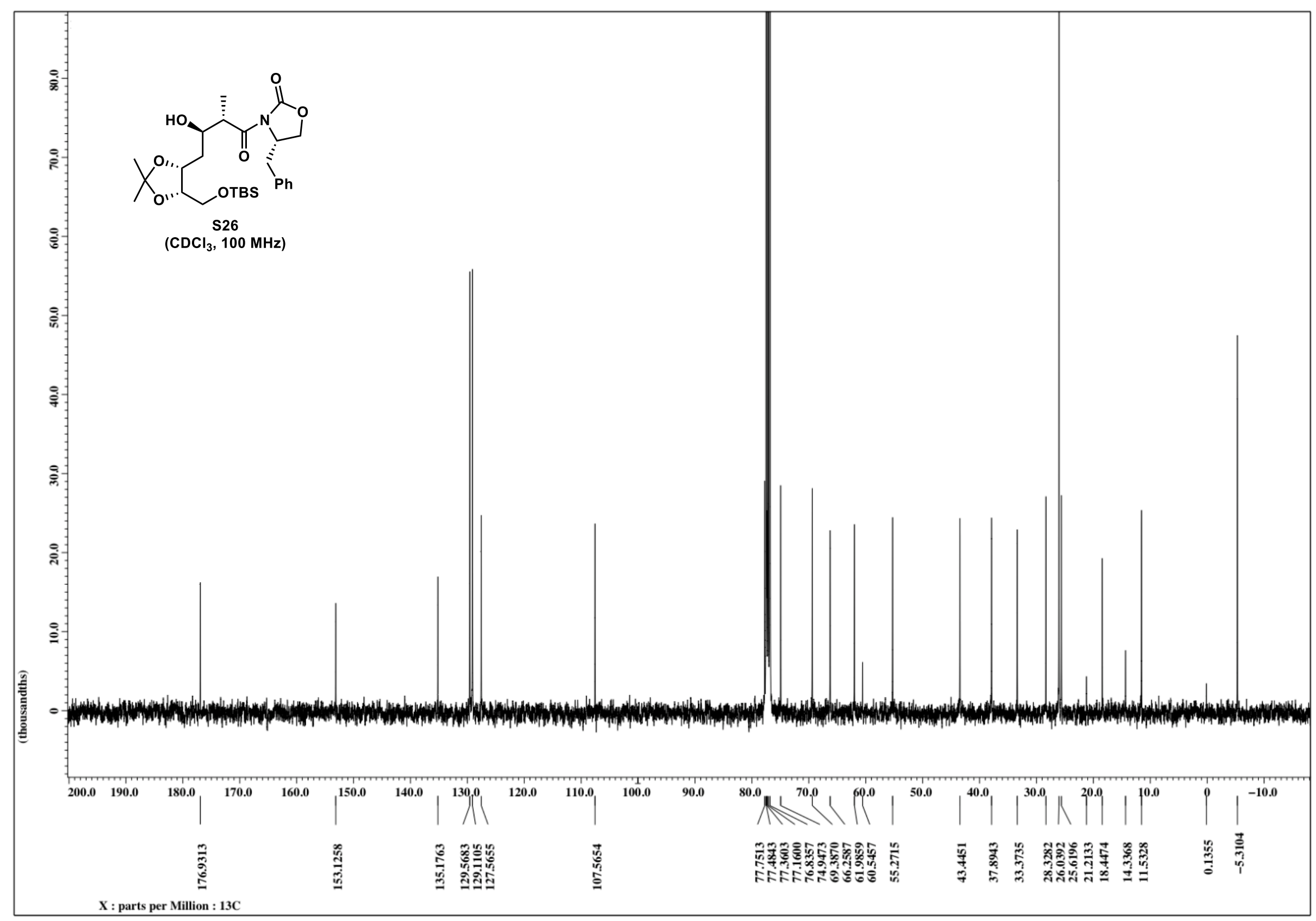

S104 


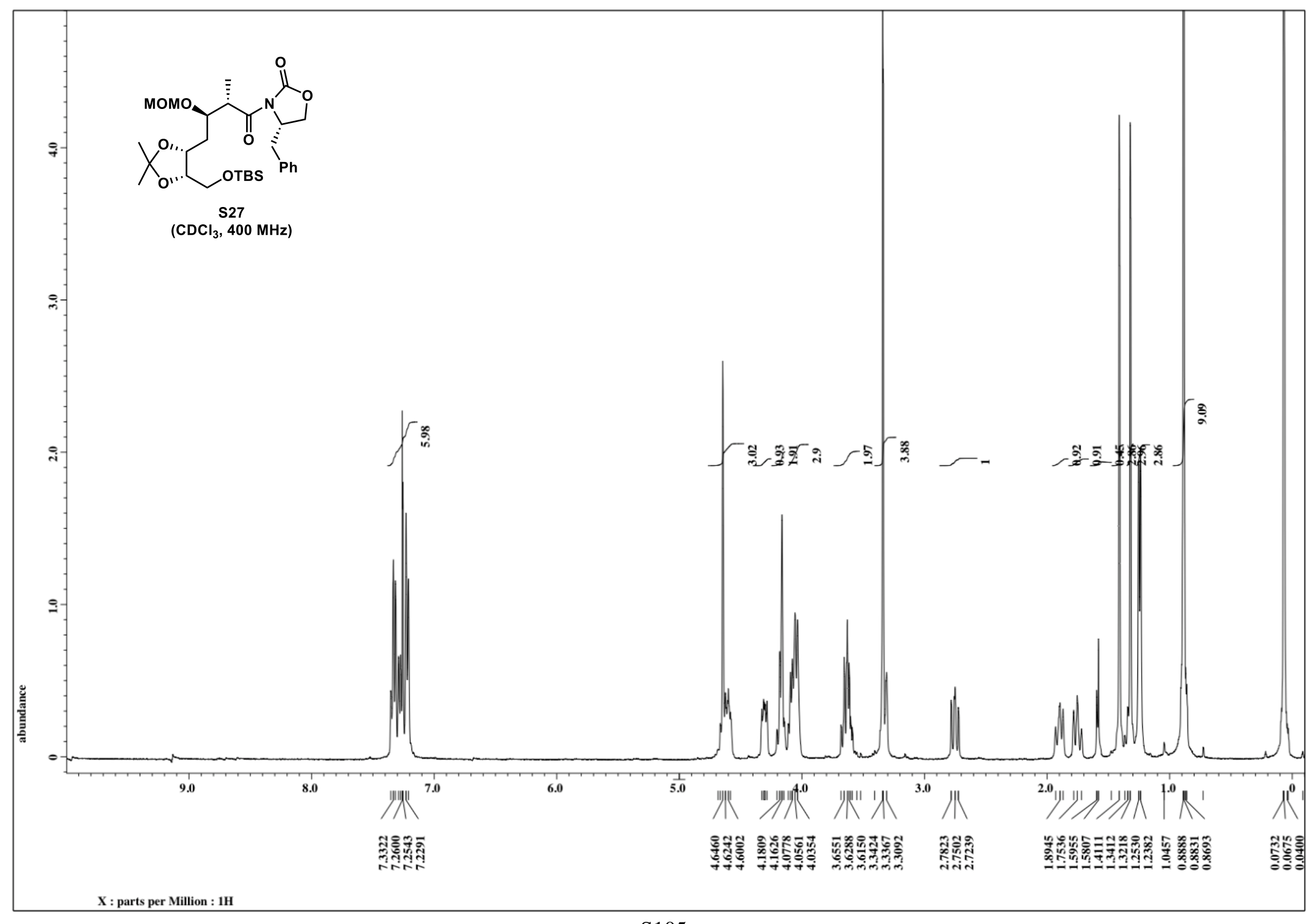




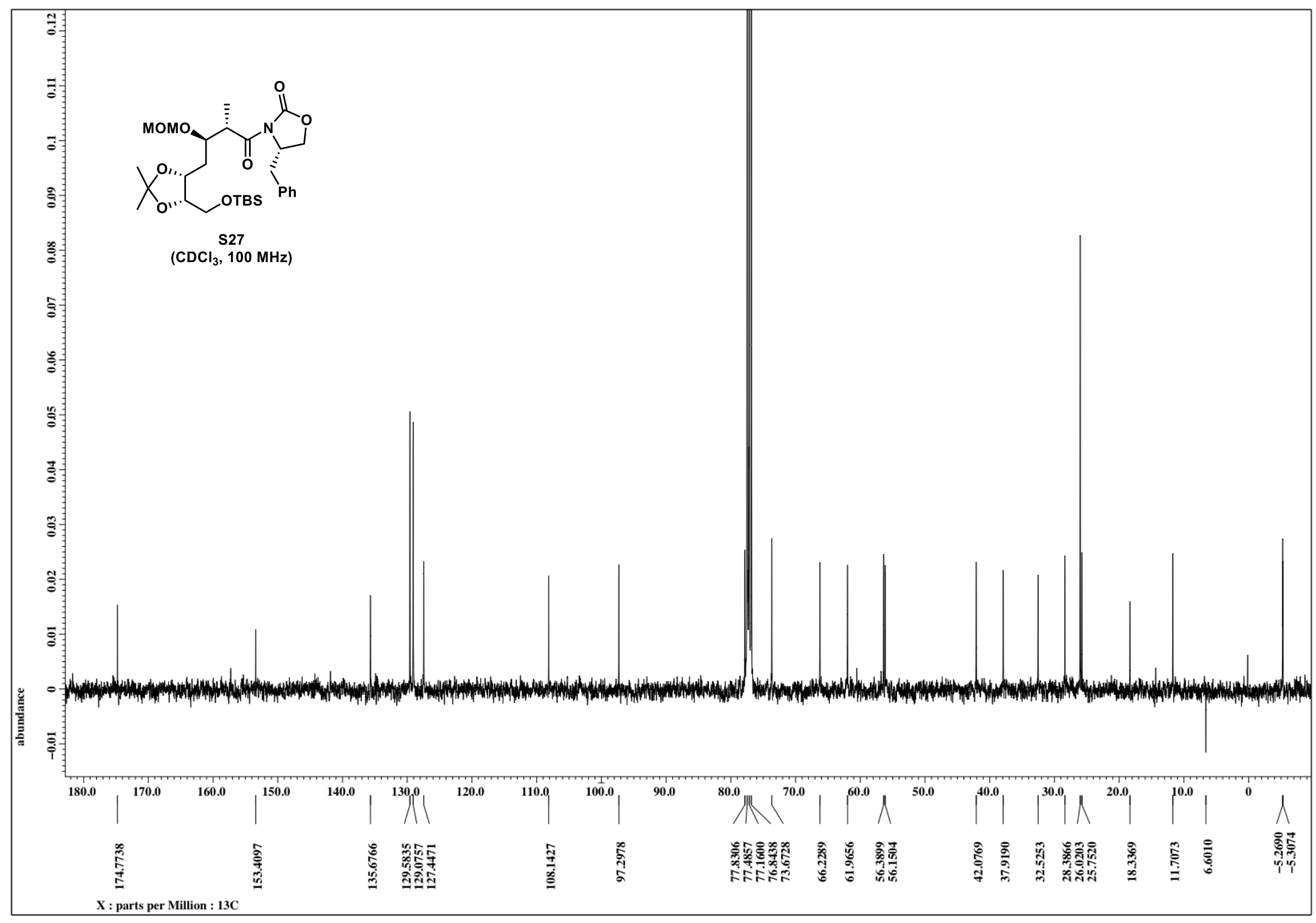




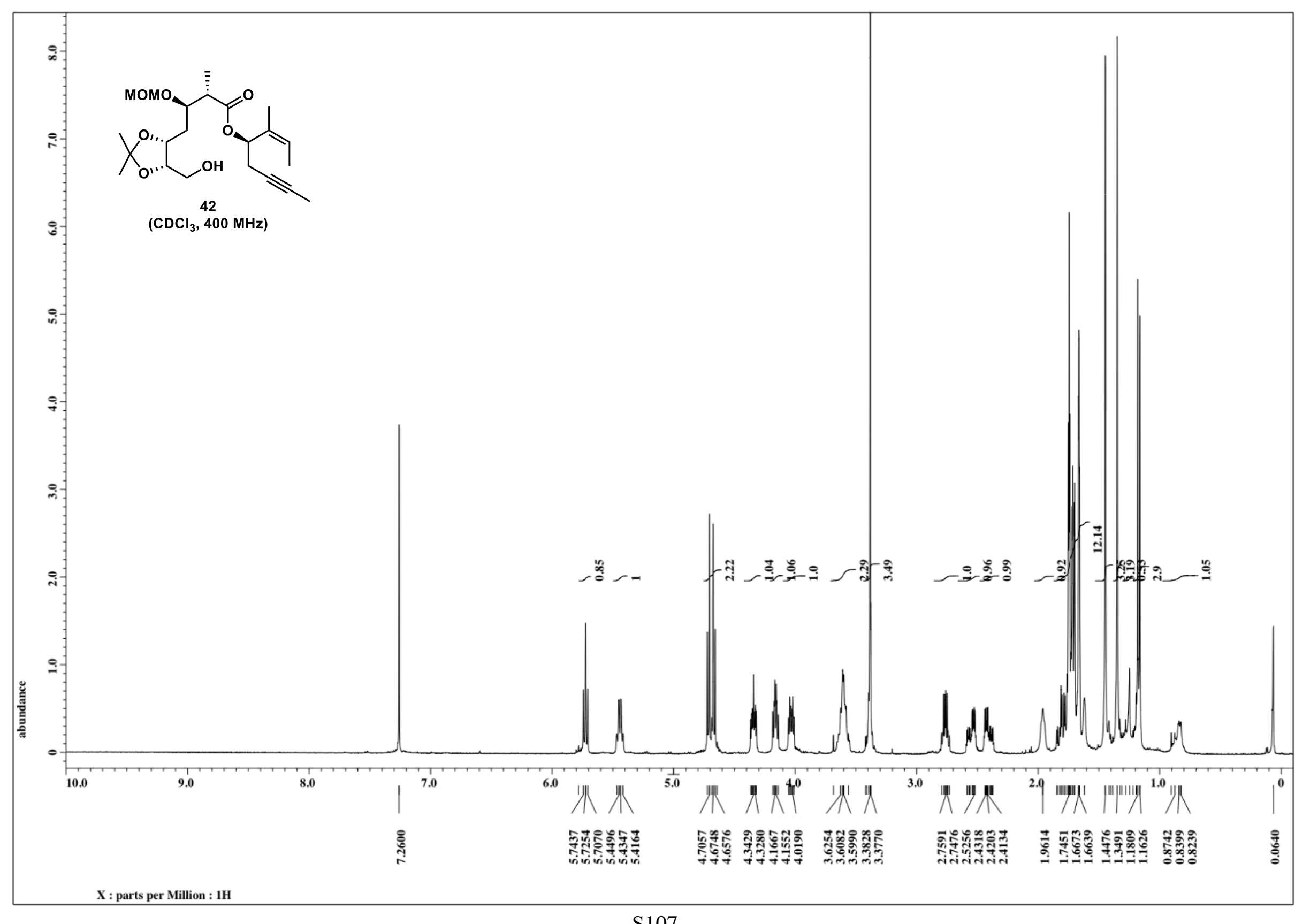




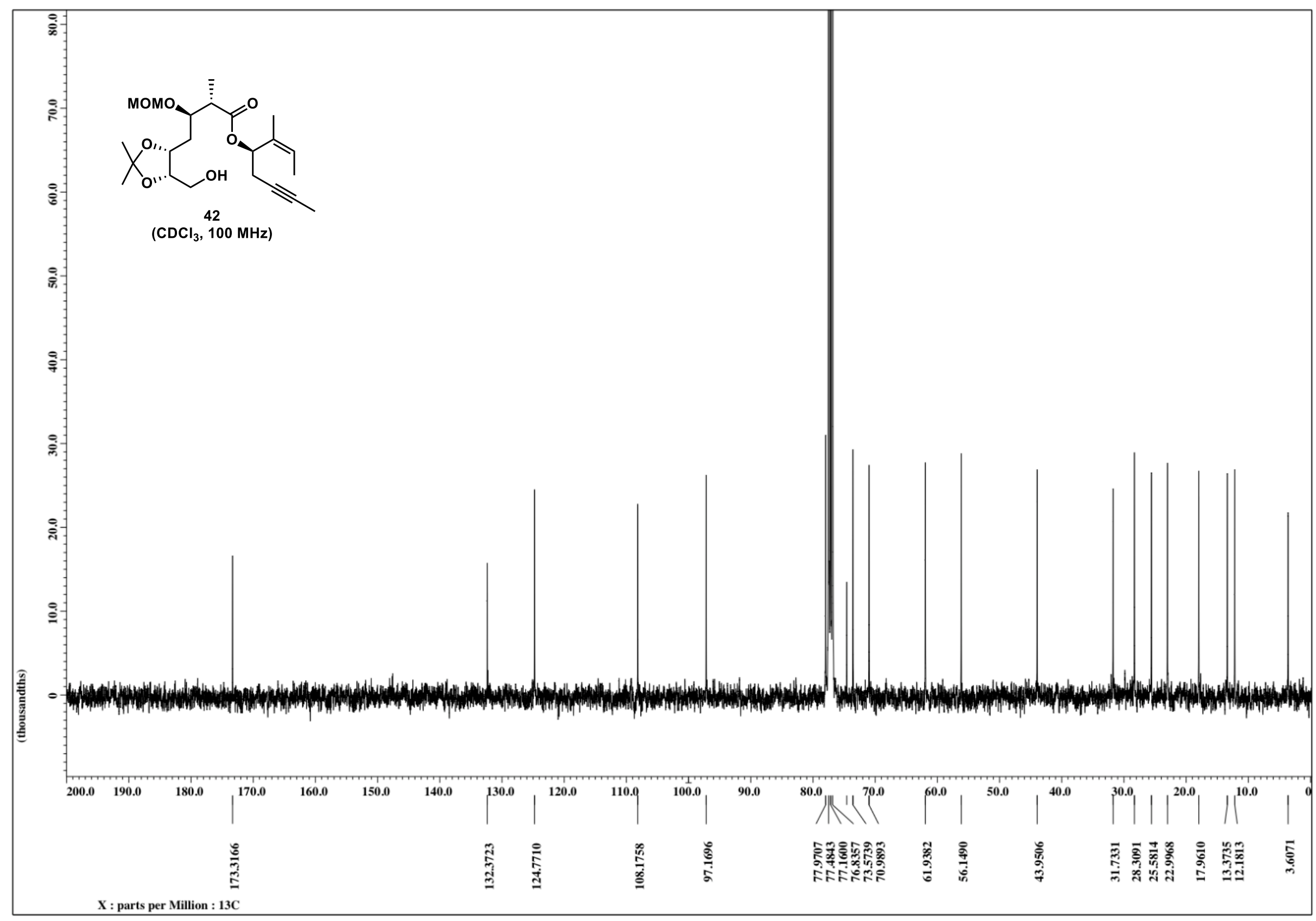




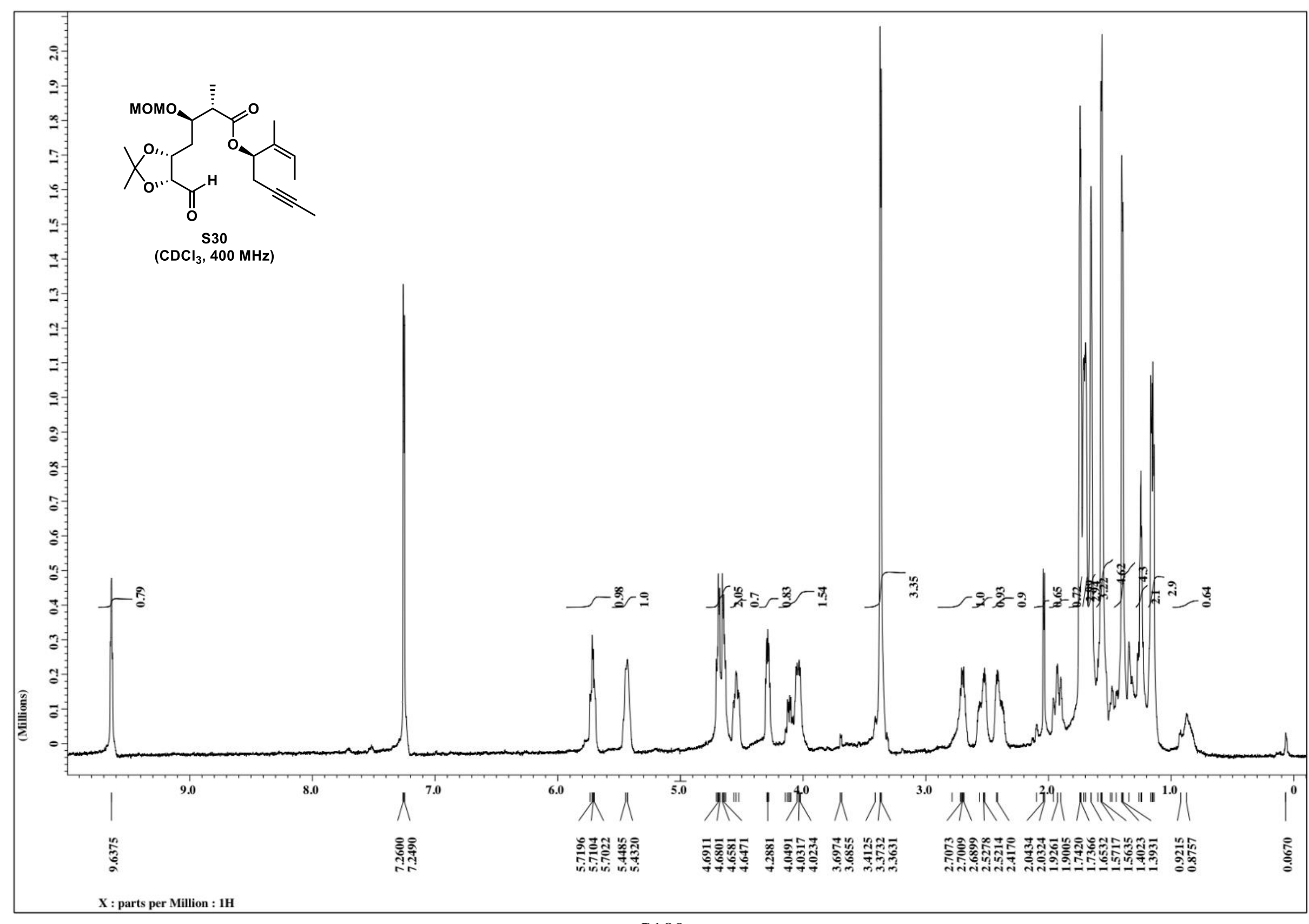




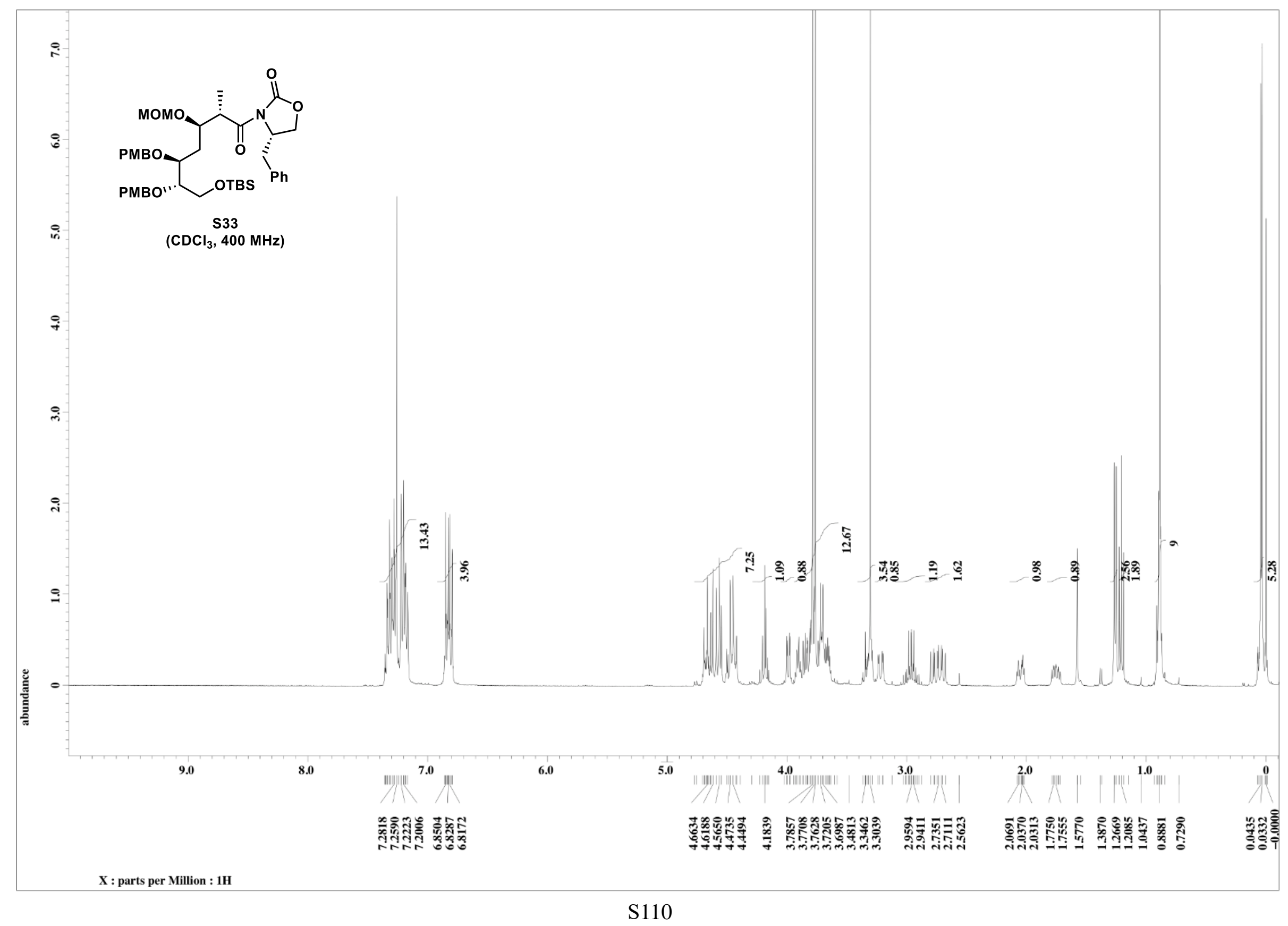




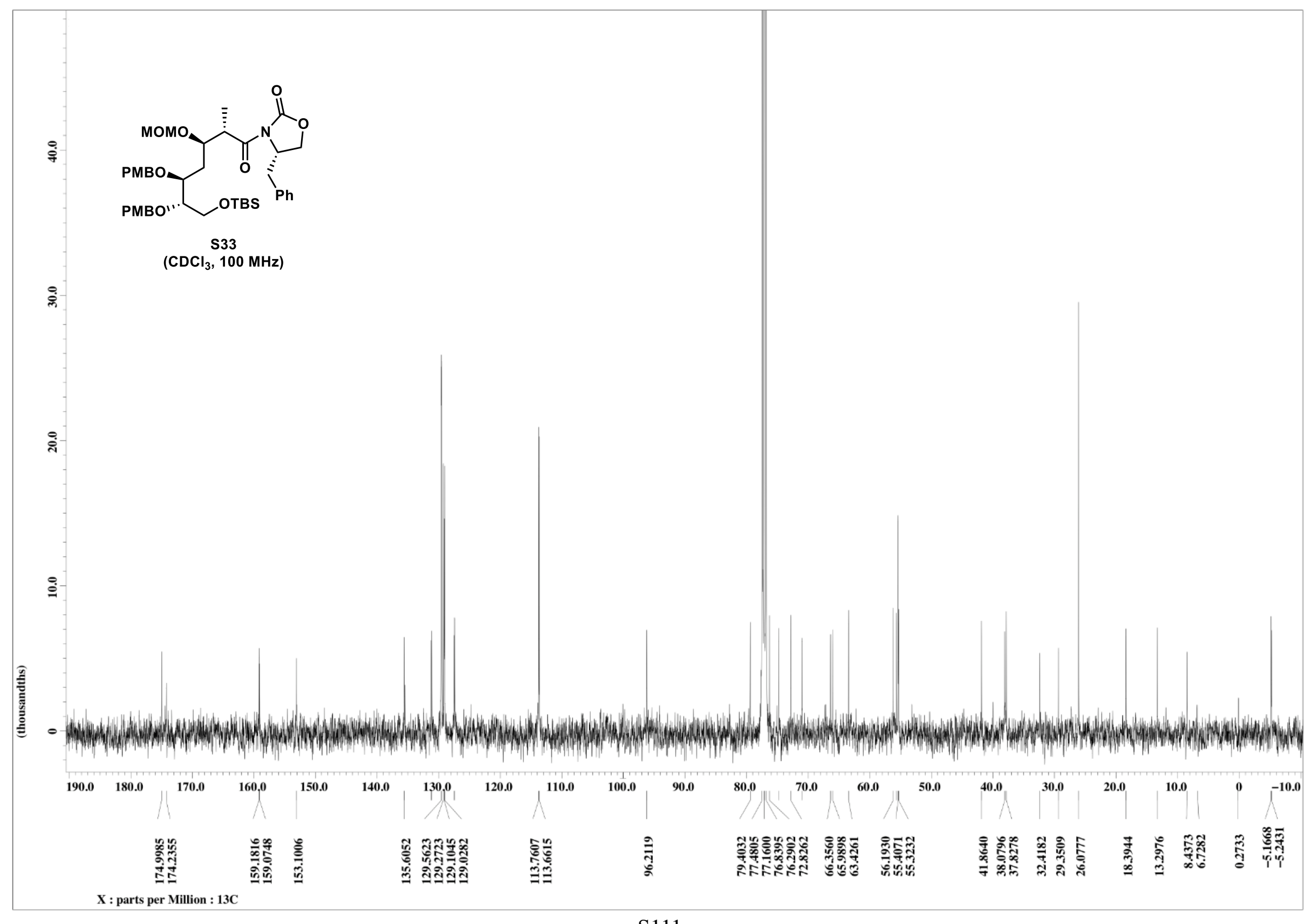




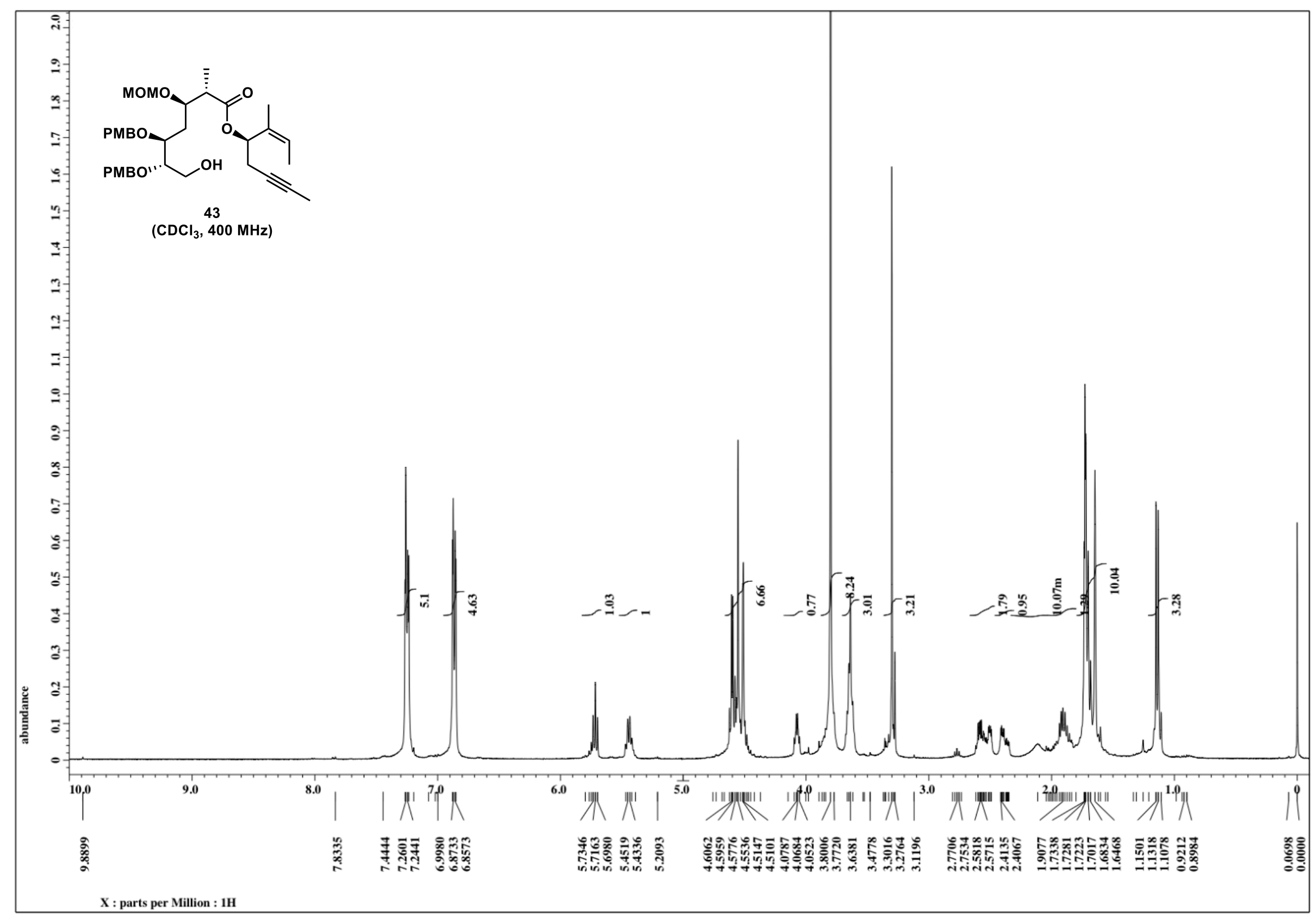




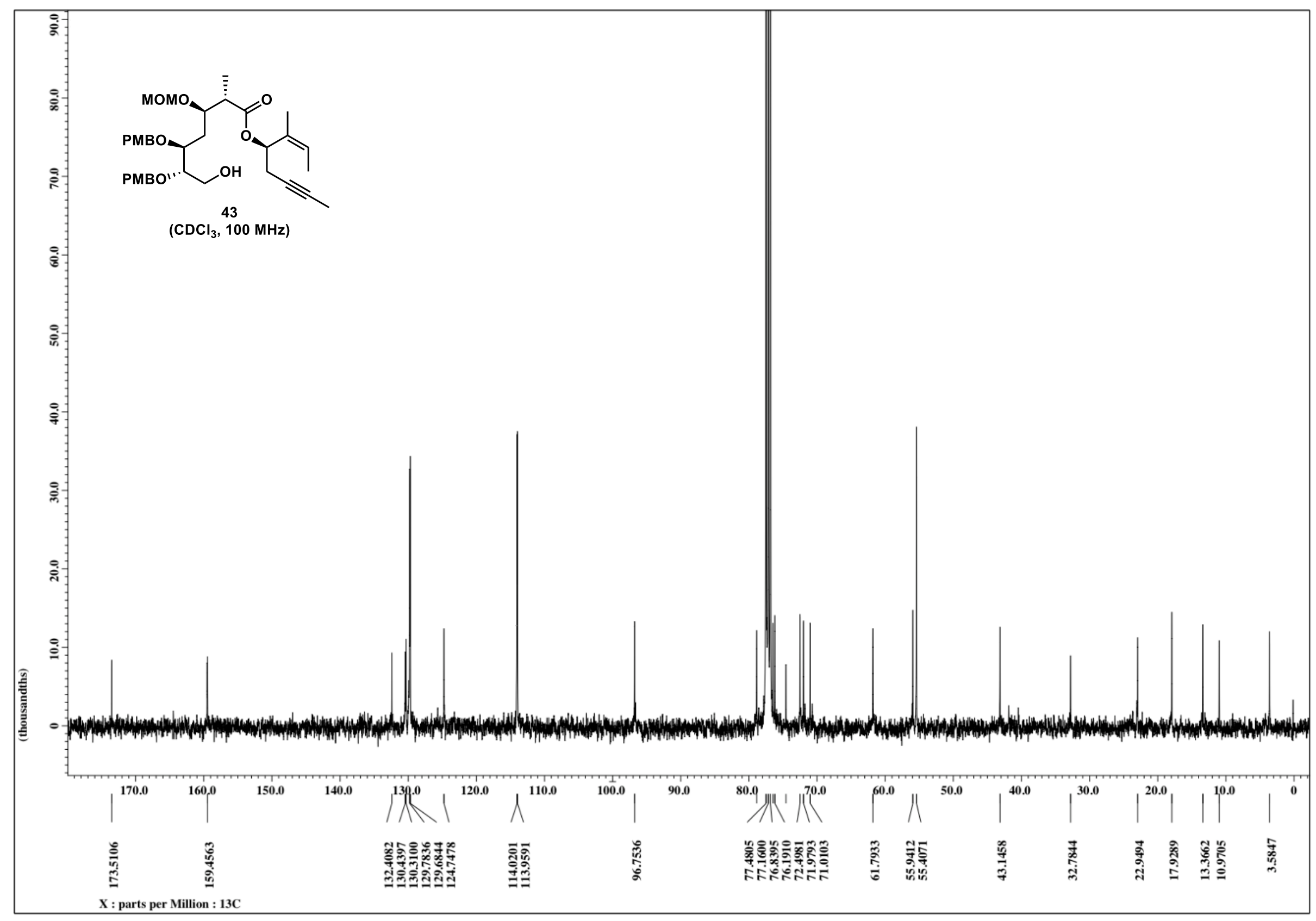




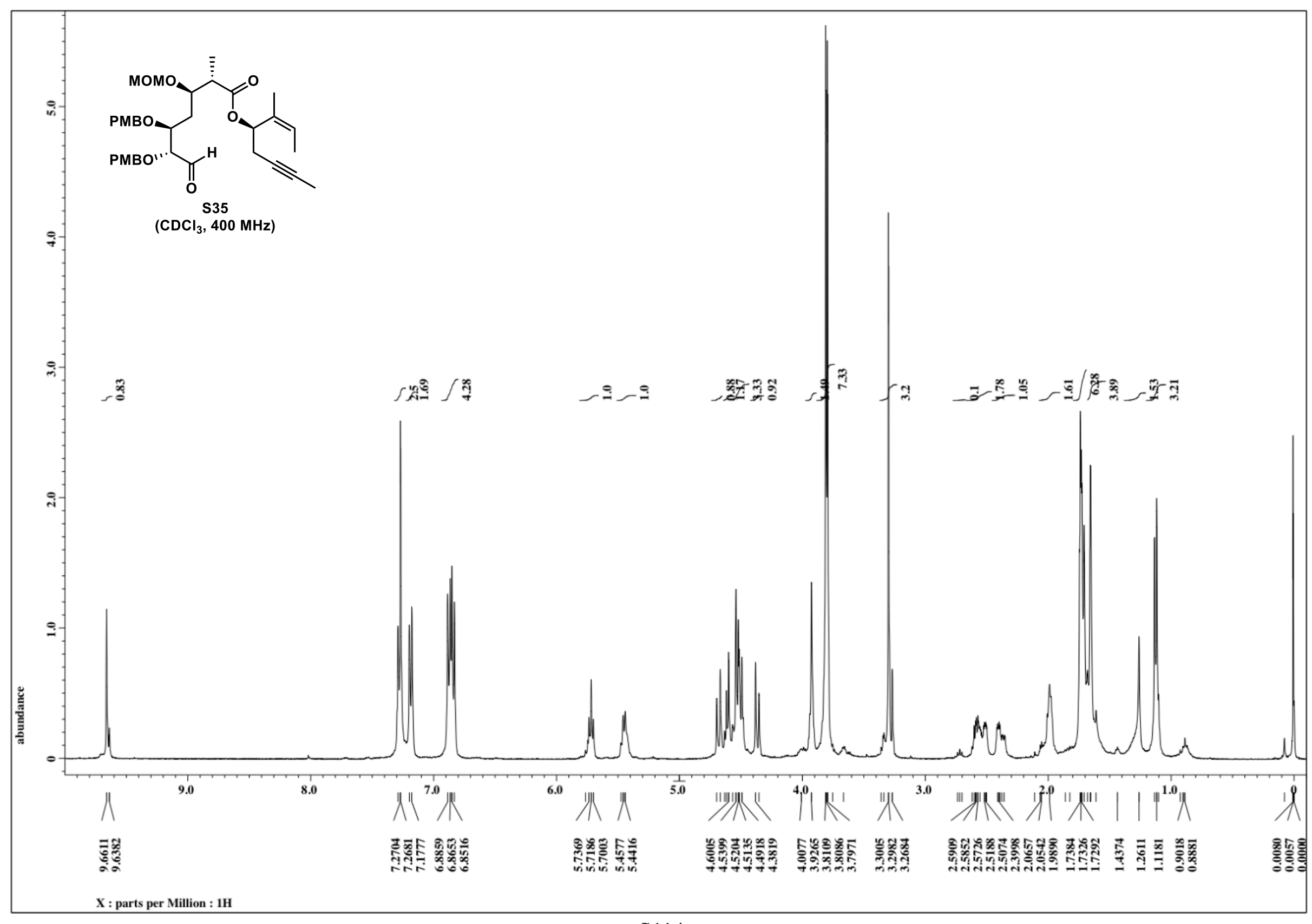




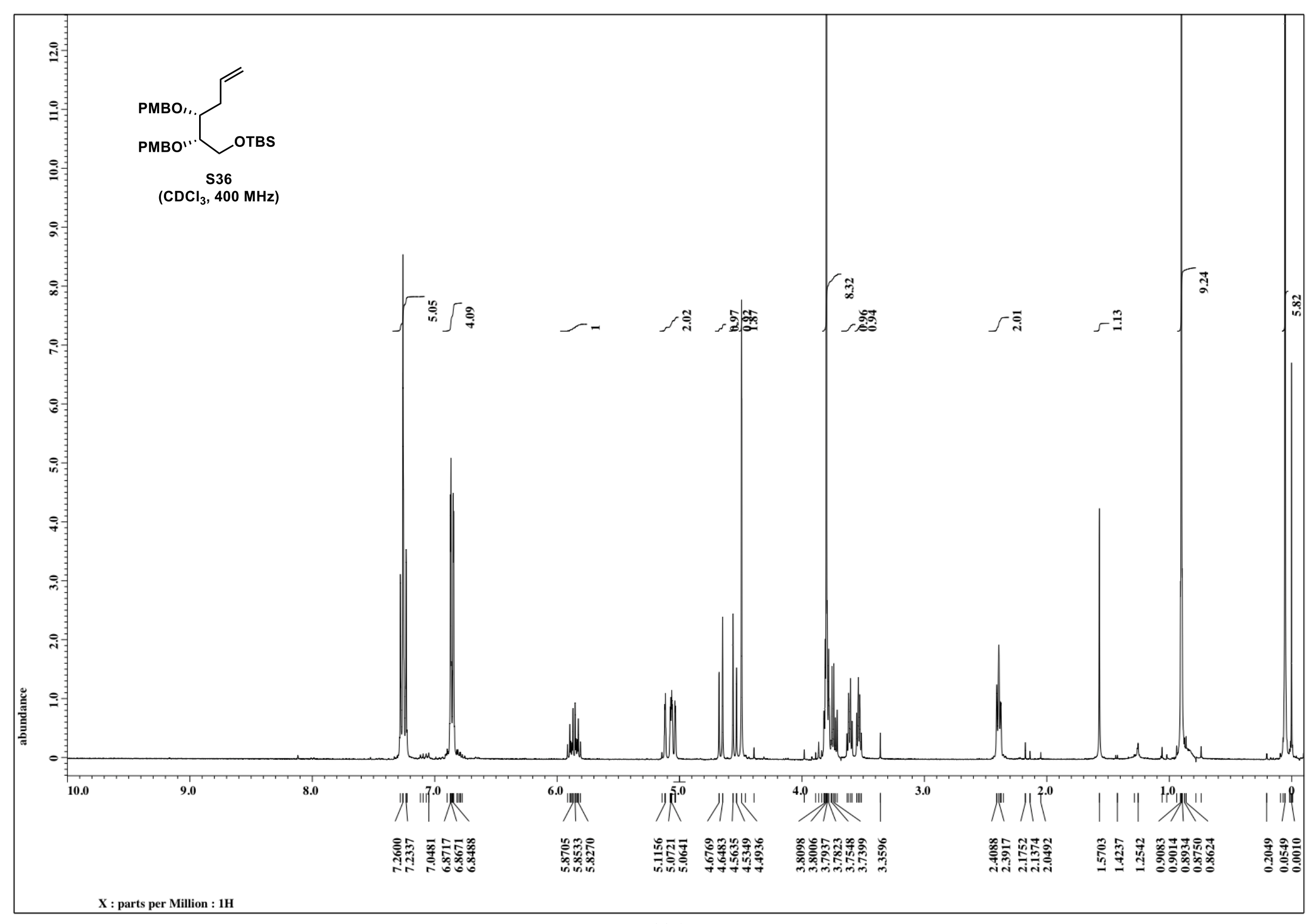




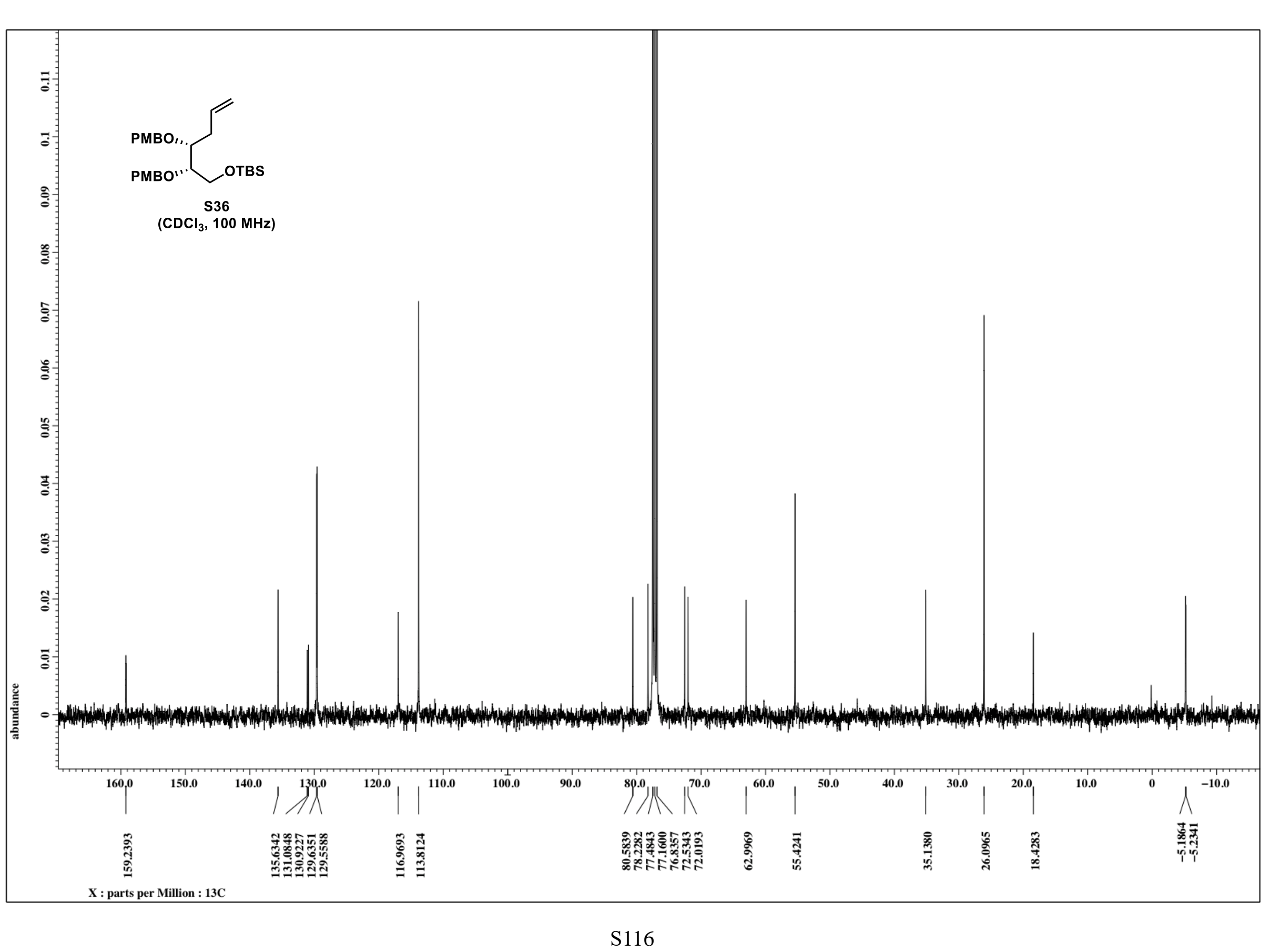




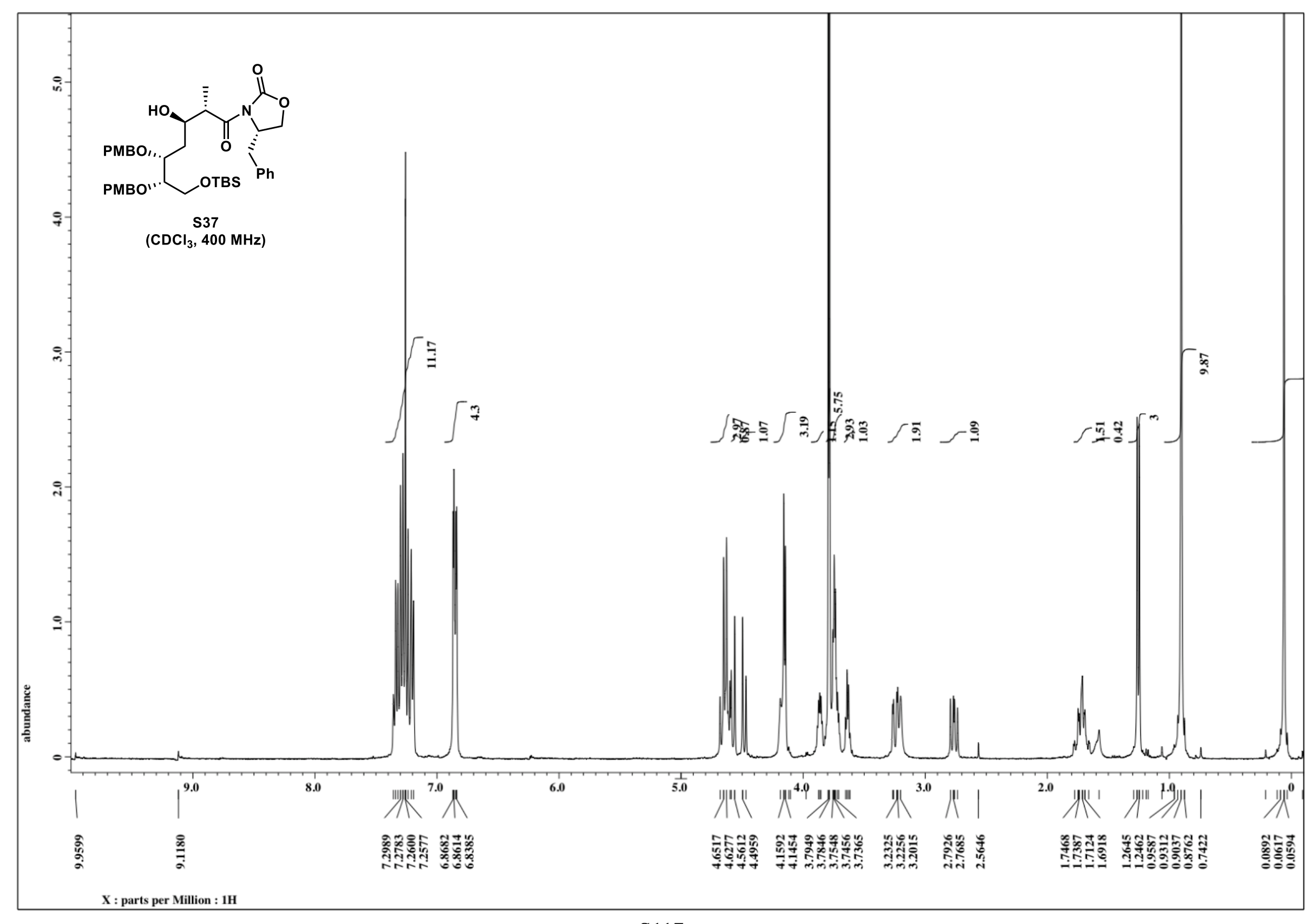




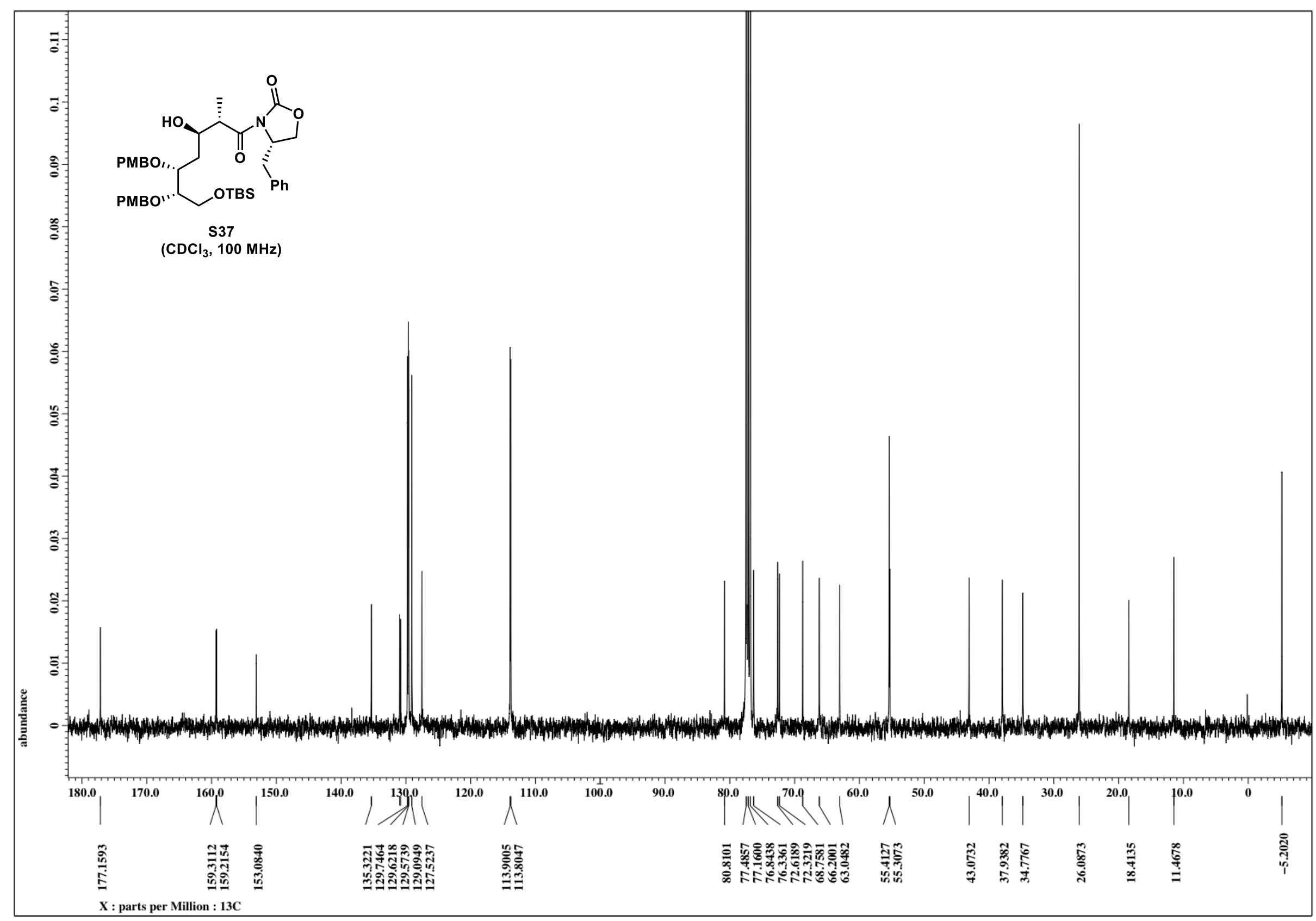




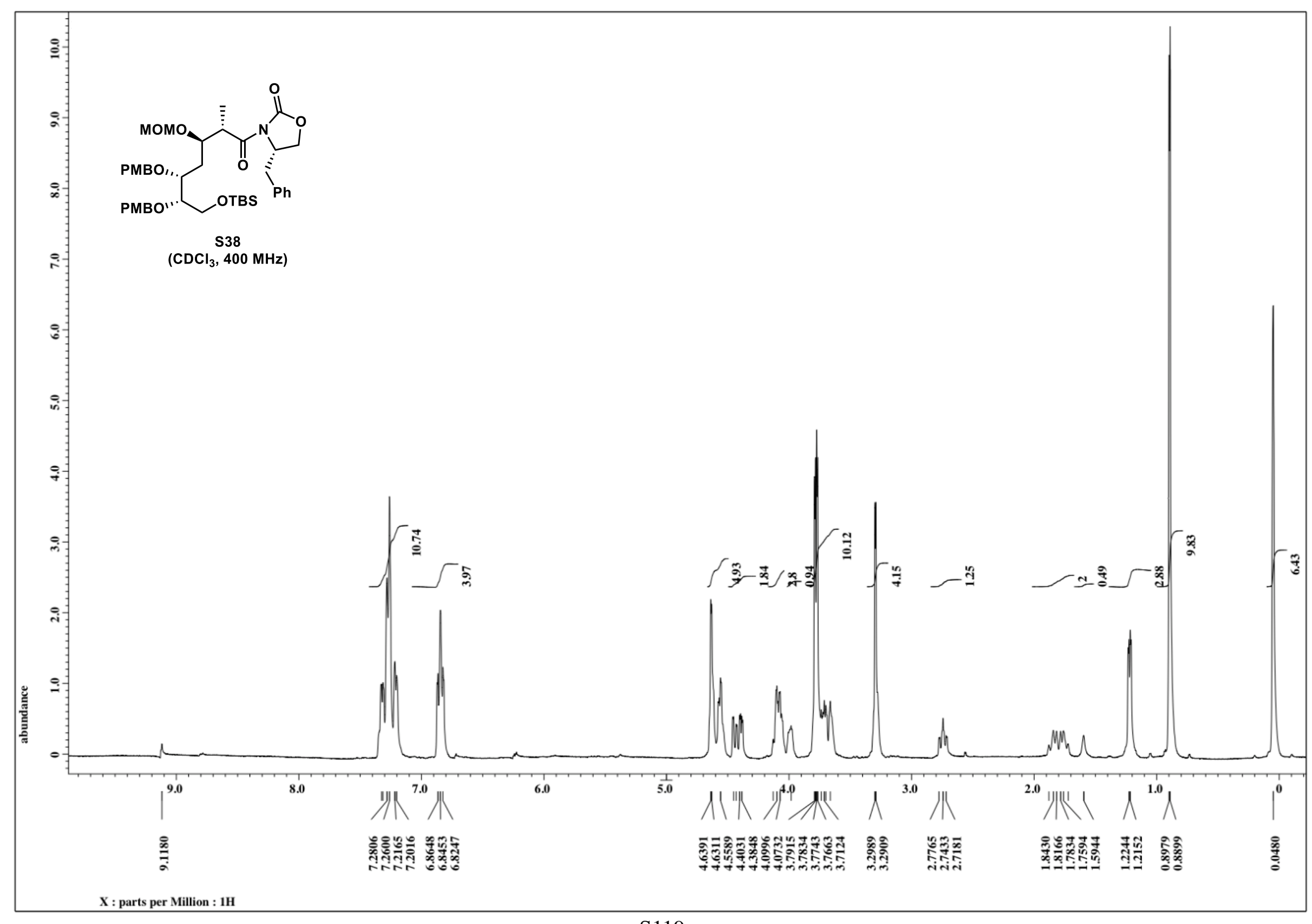




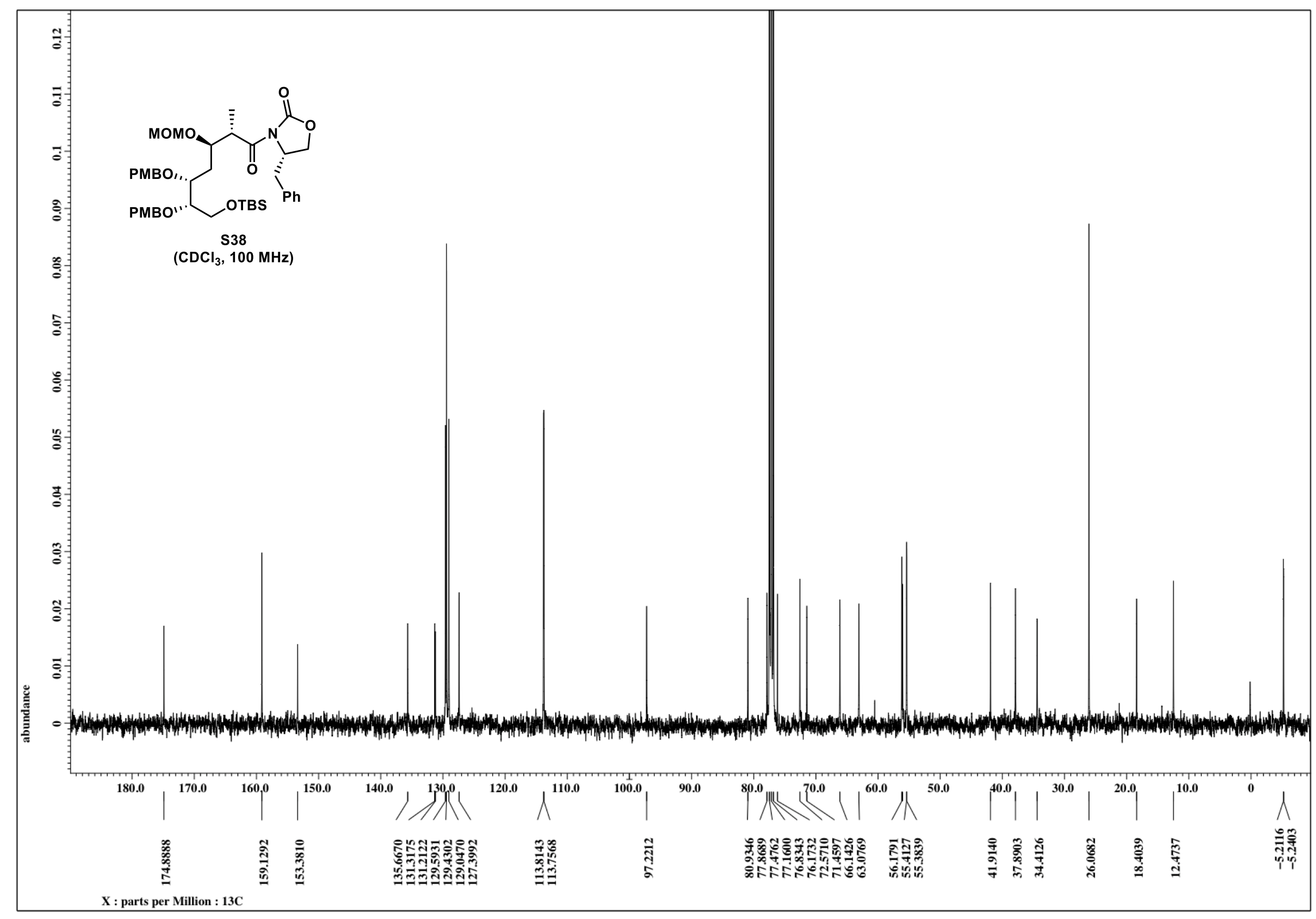

S120 


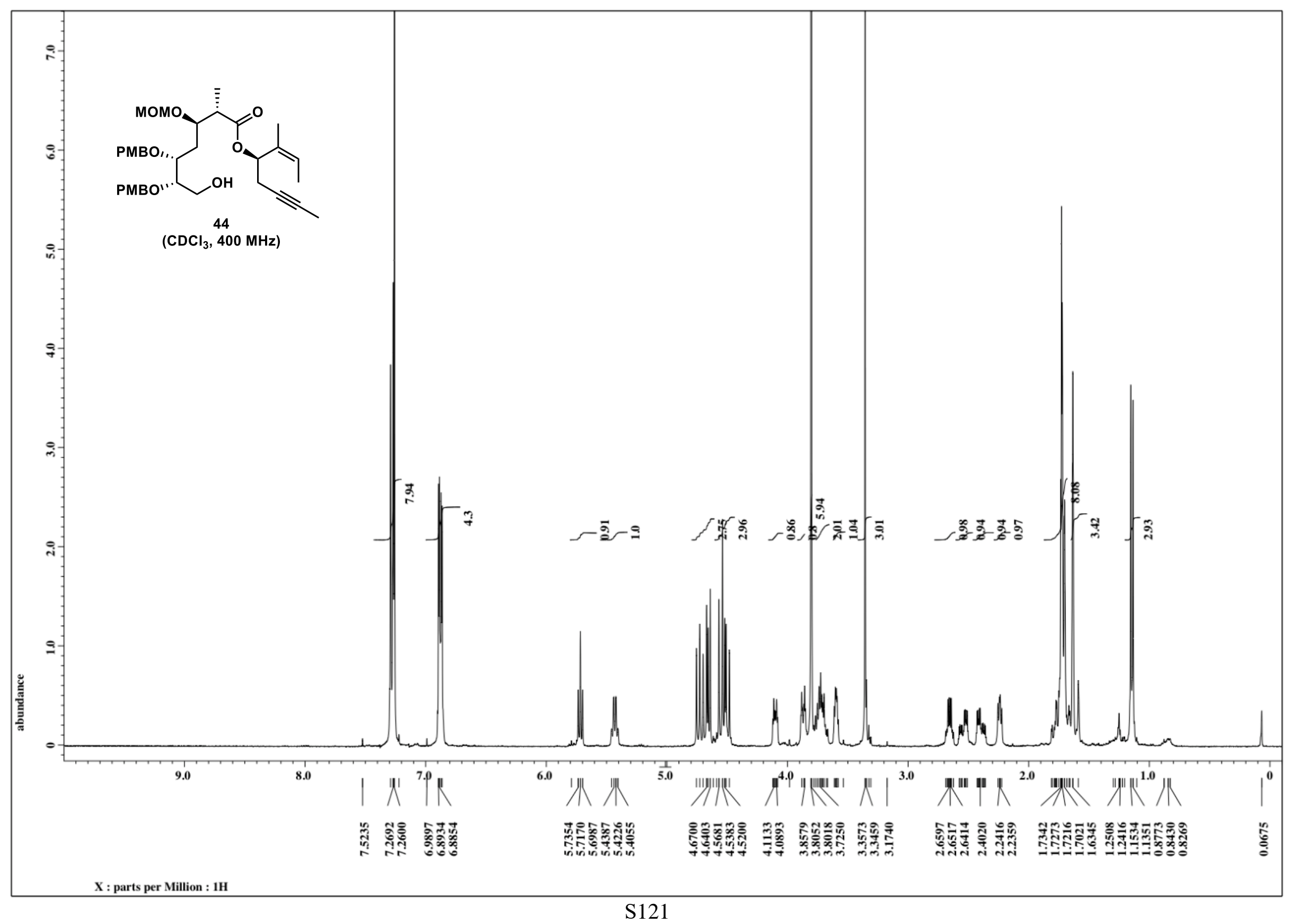




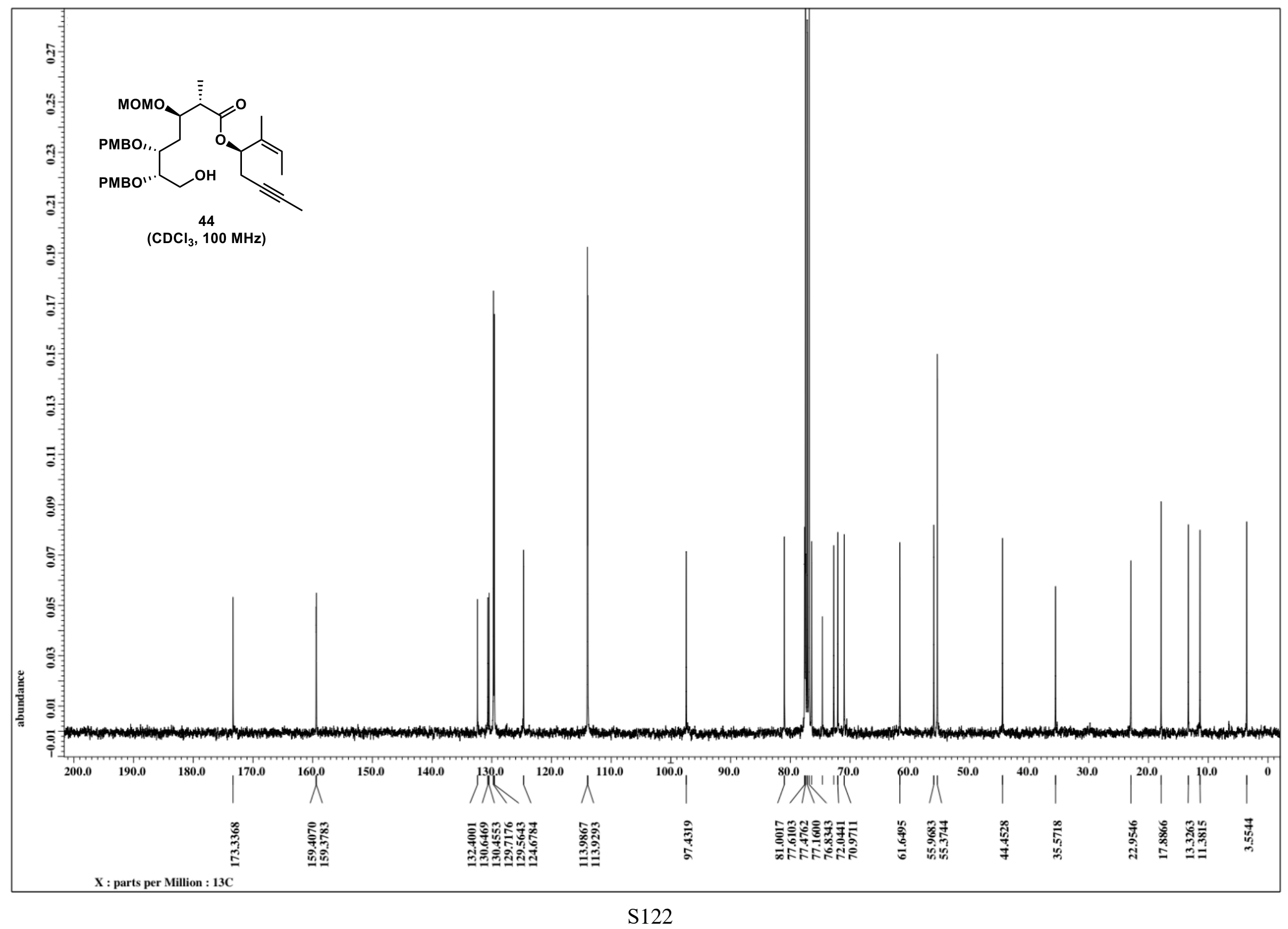




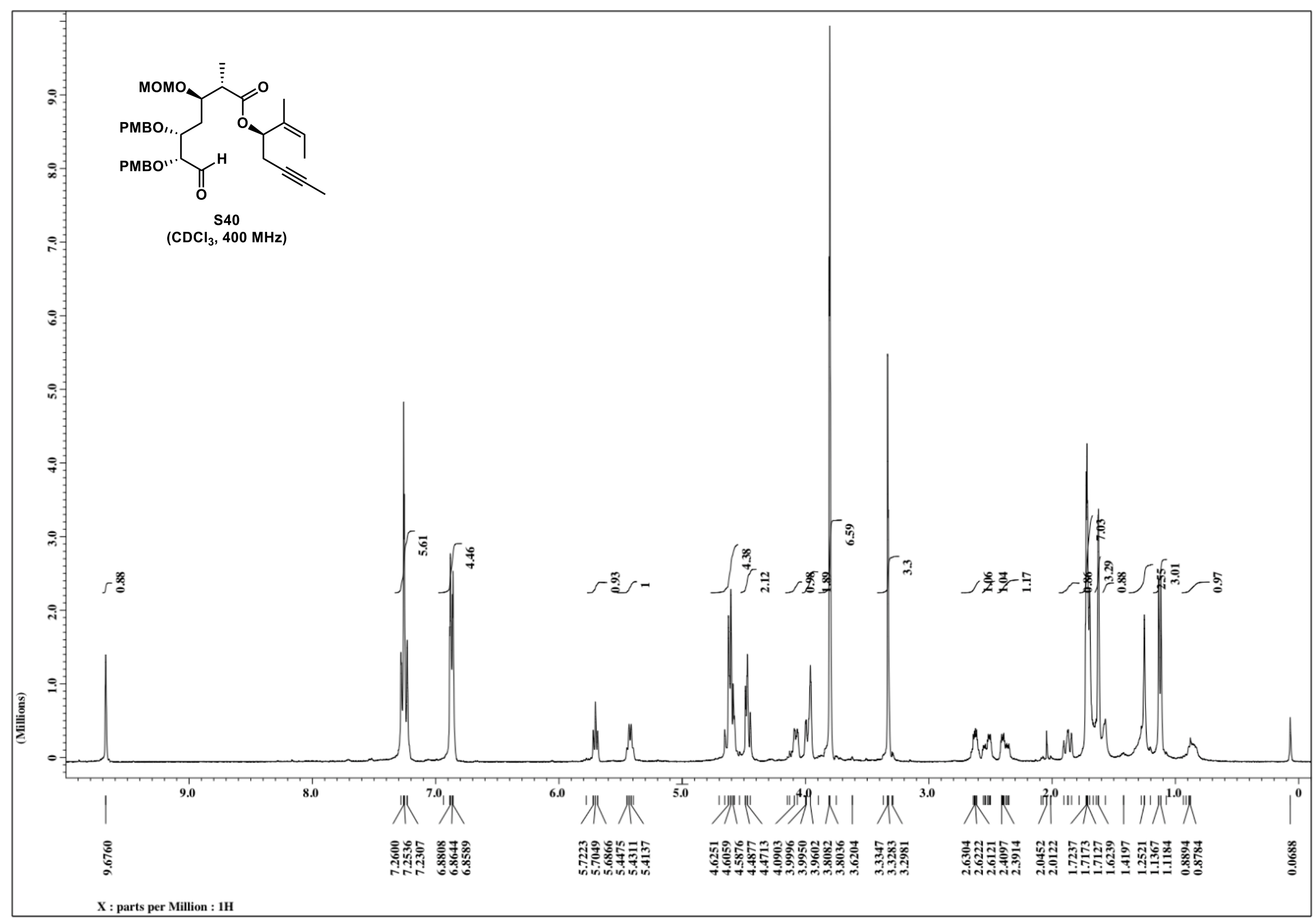

